Florida International University

FIU Digital Commons

FIU Electronic Theses and Dissertations

University Graduate School

2-19-1999

\title{
Analysis of dual-gripper manipulators and finite element analysis of the flexible laminated hose for underground storage tank cleanup
}

Badrinath Srikanth Eluri

Florida International University

DOI: $10.25148 /$ etd.FI15101295

Follow this and additional works at: https://digitalcommons.fiu.edu/etd

Part of the Mechanical Engineering Commons

\section{Recommended Citation}

Eluri, Badrinath Srikanth, "Analysis of dual-gripper manipulators and finite element analysis of the flexible laminated hose for underground storage tank cleanup" (1999). FIU Electronic Theses and Dissertations. 3141.

https://digitalcommons.fiu.edu/etd/3141

This work is brought to you for free and open access by the University Graduate School at FIU Digital Commons. It has been accepted for inclusion in FIU Electronic Theses and Dissertations by an authorized administrator of FIU Digital Commons. For more information, please contact dcc@fiu.edu. 


\title{
FLORIDA INTERNATIONAL UNIVERSITY \\ Miami, Florida
}

\begin{abstract}
ANALYSIS OF DUAL-GRIPPER MANIPULATORS AND FINITE ELEMENT ANALYSIS OF THE FLEXIBLE LAMINATED HOSE FOR UNDERGROUND STORAGE TANK CLEANUP
\end{abstract}

A thesis submitted in partial fulfillment of the requirements for the degree of MASTER OF SCIENCE in MECHANICAL ENGINEERING by

Badrinath Srikanth Eluri 
To: Dean Gordin R. Hopkins

College of Engineering

This thesis, written by Badrinath Srikanth Eluri, and entitled ANALYSIS OF DUAL-GRIPPER MANIPULATORS AND FINITE ELEMENT ANALYSIS OF THE FLEXIBLE LAMINATED HOSE FOR UNDERGROUND STORAGE TANK CLEANUP, having been approved in respect to style and intellectual content, is referred to you for judgment.

We have read this thesis and recommend that it be approved.

Dr. S.M. Lee

Dr. I.N. Tansel

Dr. Tachung Yih, Major Professor

Date of Defense: February 19, 1999

The thesis of Badrinath Srikanth Eluri is approved.

Dean Gordon R. Hopkins

College of Engineering

Dean Richard L. Campbell Division of Graduate Studies

Florida International University, 1999 


\section{DEDICATION}

I dedicate this thesis to my major professor Dr. Tachung Yih. Without his patience, understanding, and support, the completion of this work would not have been possible. 


\section{ACKNOWLEDGMENTS}

I wish to thank the members of my committee for their support and patience. Their gentle but firm direction has been most appreciated. I would like to thank my major professor, Dr. Tachung Yih. From the beginning, he had confidence in my abilities to not only complete a degree, but to complete it with excellence. His individual help and valuable suggestions have surely supported me to accomplish my master's study. His financial support from his research grants made concentrate attention on the research and study. This research project supported by the Oak Ridge National Laboratory (ORNL), the Department of Energy (DOE), is gratefully acknowledged.

I have found my coursework throughout the Curriculum and Instruction program to be stimulating and thoughtful, providing me with the tools with which to explore both past and present ideas and issues. 


\title{
ABSTRACT OF THE THESIS
}

\section{ANALYSIS OF DUAL-GRIPPER MANIPULATORS AND FINITE ELEMENT ANALYSIS OF THE FLEXIBLE LAMINATED HOSE FOR UNDERGROUND STORAGE TANK CLEANUP}

\author{
by \\ Badrinath Srikanth Eluri \\ Florida International University, 1999 \\ Miami, Florida \\ Professor Tachung Yih, Major Professor
}

The second generation long-reach manipulator - the dual-gripper Phase 3 Remote Tool Delivery (RTD) system has been developed to retrieve radioactive waste from underground storage tanks with internal structural members. The RTD is equipped with two grippers, one at each end of the system, to grasp the structural member. A vertical translation mechanism is also added to the system that enables the vertical motion inside the tank. This special feature provides greater dexterity and flexibility. Two operating open-chain configurations are considered and modeled. The first configuration is that gripper 1 grasps the structural member and gripper 2 is free to perform a designated task. The other configuration is that gripper 2 grasps the structural member and gripper 1 is free to move. The kinematics, dynamics and simulation of the system are emphasized.

Finite Element Analysis (FEA) of the flexible laminated hose used to convey radioactive waste materials was investigated to learn about its structural behavior. The hose is considered relatively fixed at one end (the outlet) and subjected to different loading conditions at the inlet. Liquid (mixture of water and sludge) with high viscosity is sucked through the hose using a vacuum pressure.

The software package employed for FEA is ANSYS 5.2. A total of 200 cases, with different $\mathrm{X}, \mathrm{Y}$ and $\mathrm{Z}$ loading conditions at the inlet, for the hose under the consideration with or without vacuum pressure have been analyzed. Numerical results and the ANSYS graphic outputs provided for each case include the deformation, stress distribution and strain distribution throughout the hose. 
CHAPTER

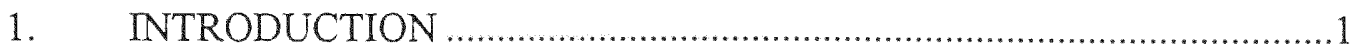

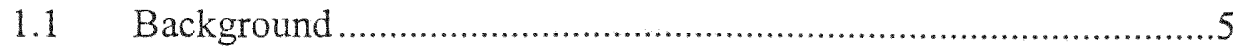

2. KINEMATICS AND DYNAMICS OF DUAL-GRIPPER

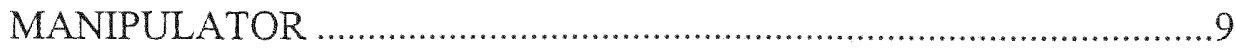

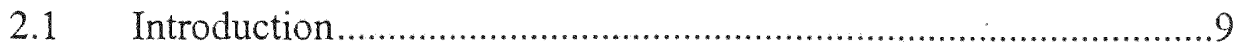

2.2 Review of C-B Notation .............................................................. 10

2.3 Modeling of Dual-gripper Manipulator Using C-B Notation ........12

2.3.1 The Phase 2 RTD System .................................................12

2.3.2 The Phase 3 RTD System ................................................19

2.4 Vertical Translation Mechanism.................................................28

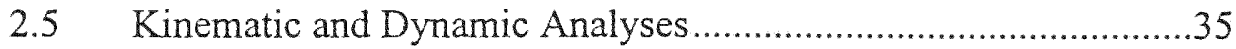

2.5.1 Simulation of the Phase 3 RTD System...............................36

2.5.2 Numerical Examples ........................................................52

3. FINTTE ELEMENT ANALYSIS OF THE FLEXIBLE LAMINATED

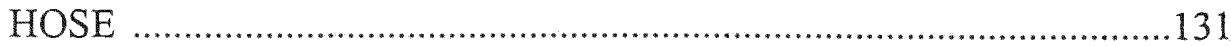

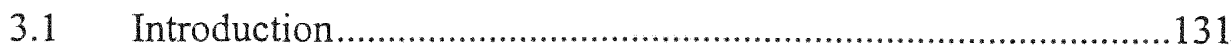

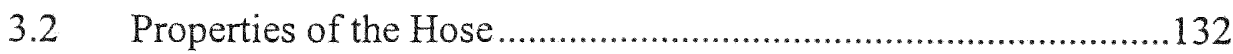

3.3 Structural Analysis of the Hose ..................................................133

3.3.1 Construction of the hose and mesh generation ..................134

3.3.2 Boundary conditions and applied loads .............................138

3.4 Finite Element Analysis...........................................................140

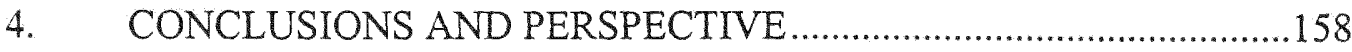

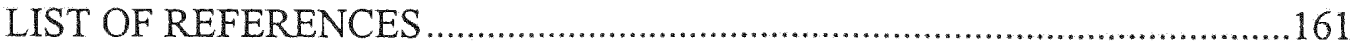




\section{LIST OF FIGURES}

FIGURE

Page No.

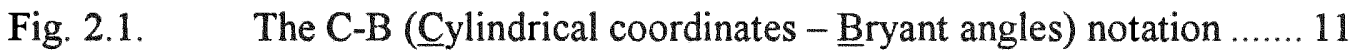

Fig. 2.2. The fixed frame of reference for Phase 2 RTD system .............. 13

Fig. 2.3. Definition of joint 2, Phase 2 RTD system, for arm deployment . 15

Fig. 2.4. Definition of joint axes for the Phase 2 RTD system ................ 16

Fig. 2.5. Assignment of joint and link parameters for phase 3 RTD system20

Fig. 2.6. Case 1-Gripper 1 grasps the structural member in the tank ...... 24

Fig. 2.7. Case 2-Gripper 2 grasps the structural member in the tank ...... 24

Fig. 2.8. The vertical translation mechanism (VTM) ............................ 29

Fig. 2.9. Analysis of VTM while gripper 1 is fixed .............................. 31

Fig. 2.10. Analysis of VTM while gripper 2 is fixed ..................................33

Fig. 2.11a. The RTD system following a straight line trajectory - initial ...... 38

Fig. 2.11b. The RTD system following a straight line trajectory - final ....... 39

Fig. 2.12a. The RTD system following a straight line trajectory - initial ..... 40

Fig. 2.12b. The RTD system following a straight line trajectory - final ....... 41

Fig. 2.13a. The RTD system following a straight line trajectory - initial ..... 42

Fig. 2.13b. The RTD system following a straight line trajectory $-\mathrm{t} 1$ to $\mathrm{t} 2 \ldots .43$

Fig. 2.13c. The RTD system following a straight line trajectory $-\mathrm{t} 2$ to $\mathrm{t} 3 \ldots .44$

Fig. $2.13 \mathrm{~d}$. The RTD system following a straight line trajectory $-\mathrm{t} 3$ to $t 4 \ldots, 45$

Fig. 2.13e. The RTD system following a straight line trajectory - final ....... 46

Fig. 2.14a. The RTD system following a straight line trajectory - initial ..... 47 
Fig. 2.14b. The RTD system following a straight line trajectory $-\mathrm{t} 1$ to $\mathrm{t} 2 \ldots .48$

Fig. 2.14c. The RTD system following a straight line trajectory $-\mathrm{t} 2$ to $\mathrm{t} 3 \ldots .49$

Fig. 2.14d. The RTD system following a straight line trajectory $-\mathrm{t} 3$ to $t 4 \ldots .50$

Fig. 2.14e. The RTD system following a straight line trajectory - final .......51

Fig. 2.15a. Gripper 2 angular displacement (Example 1) .......................... 54

Fig. 2.15b. Gripper 2 angular velocity (Example 1) ................................. 55

Fig. 2.15c. Gripper 2 angular acceleration (Example 1) ............................. 56

Fig. 2.15d. Torque of joint 2' (Example 1) ……....................................... 57

Fig. 2.16a. Angular displacement of joint 2' (Example 1) .........................58

Fig. 2.16b. Angular velocity of joint 2' (Example 1) ..................................59

Fig. 2.16c. Angular acceleration of joint 2' (Example 1) ........................... 60

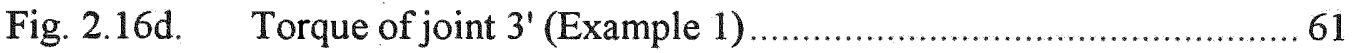

Fig. 2.17a. Angular displacement of joint 3' (Example 1) ......................... 62

Fig. 2.17b. Angular velocity of joint 3 ' (Example 1) .................................... 63

Fig. 2.17c. Angular acceleration of joint 3' (Example 1) ............................64

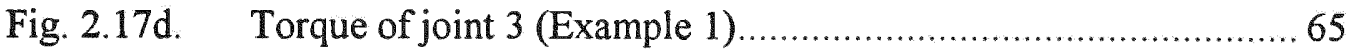

Fig. 2.18a. Angular displacement of joint 3 (Example 1) ........................... 66

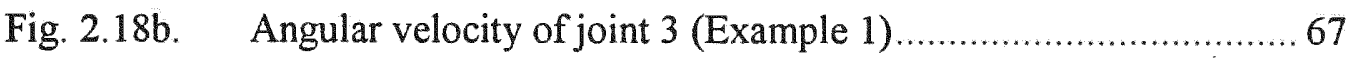

Fig. 2.18c. Angular acceleration of joint 3 (Example 1) ..............................68

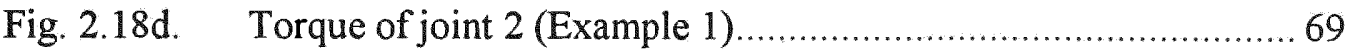

Fig. 2.19a. Gripper 2 angular displacement (Example 2) ….................. 71

Fig. 2.19b. Gripper 2 angular velocity (Example 2) …............................ 71

Fig. 2.19c. Gripper 2 angular acceleration (Example 2) .......................... 73 
Fig. 2.19d. Torque of joint 2 (Example 2) ........................................ 74

Fig. 2.20a. Angular displacement of joint 2 (Example 2) ........................ 75

Fig. 2.20b. Angular velocity of joint 2 (Example 2) ................................ 76

Fig. 2.20c. Angular acceleration of joint (Example 2) ............................. 77

Fig. 2.20d. Torque of joint 3' (Example 2) …......................................... 78

Fig. 2.21a Angular displacement of joint 3 (Example 2) ........................ 79

Fig. 2.21b. Angular velocity of joint 3 (Example 2) ................................. 80

Fig. 2.21c. Angular acceleration of joint 3 (Example 2) ........................... 81

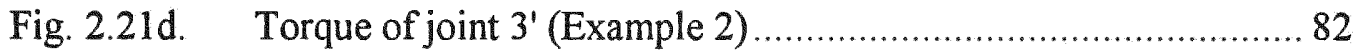

Fig. 2.22a. Angular displacement of joint 3' (Example 2) ....................... 83

Fig. 2.22b. Angular velocity of joint 3' (Example 2) ................................ 84

Fig. 2.22c. Angular acceleration of joint 3' (Example 2) …....................... 85

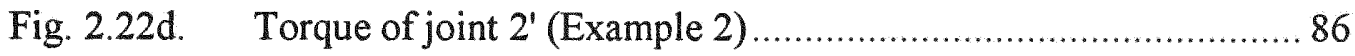

Fig. 2.23a. Gripper 2 angular displacement (Example 3) ........................ 89

Fig. 2.23b. Gripper 2 angular velocity (Example 3) _................................ 90

Fig. 2.23c. Gripper 2 angular acceleration (Example 3) .......................... 91

Fig. 2.23d. Torque of joint 2' (Example 3) …......................................... 92

Fig. 2.24a. Angular displacement of joint 2' (Example 3) ........................ 93

Fig. 2.24b. Angular velocity of joint 2' (Example 3) ................................ 94

Fig. 2.24c. Angular acceleration of joint 2'(Example 3) ............................ 95

Fig. 2.24d. Torque of joint 3' (Example 3) .......................................... 96

Fig. 2.25a. Angular velocity of joint 3 (Example 3) ............................... 97

Fig. 2.25b. Angular velocity of joint 3 (Example 3) f................................ 98 
Fig. 2.25c. Angular acceleration of joint 3 (Example 3) ......................... 99

Fig. 2.25d. Torque of joint 3 (Example 3) ........................................... 100

Fig. 2.26a. Angular displacement of VTM (Example 3) ........................ 101

Fig. 2.26b. Angular velocity of VTM (Example 3) ................................ 102

Fig. 2.26c. Angular acceleration of VTM (Example 3) ............................ 103

Fig. 2.26d. Joint torque of VTM (Example 3) ..................................... 104

Fig. 2.27a. Angular displacement of joint 3 (Example 3) ........................ 105

Fig. 2.27b. Angular velocity of joint 3 (Example 3) .............................. 106

Fig. 2.27c. Angular acceleration of joint 3 (Example 3) .......................... 107

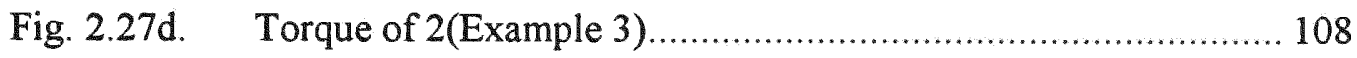

Fig. 2.28a. Gripper 1 angular displacement (Example 4) ....................... 109

Fig. 2.28b. Gripper 2 angular velocity (Example 4) ............................. 112

Fig. 2.28c. Gripper 2 angular acceleration (Example 4) ........................ 113

Fig. 2.28d. Torque of joint 2 (Example 4) ......................................... 114

Fig. 2.29a. Angular displacement of VTM (Example 4) ....................... 115

Fig. 2.29b. Angular velocity of VTM (Example 4) ............................... 116

Fig. 2.29c. Angular acceleration of VTM (Example 4) ........................ 117

Fig. 2.29d. Torque of VTM (Example 4) f............................................... 118

Fig. 2.30a. Angular displacement of joint 2 (Example 4) ....................... 119

Fig. 2.30b. Angular velocity of joint 2 (Example 4) ............................. 120

Fig. 2.30c. Angular acceleration of joint 2 (Example 4) ......................... 121

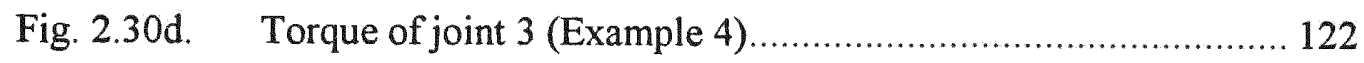

Fig. 2.31a. Angular displacement of joint 3 (Example 4) ....................... 123 
Fig. 2.31b. Angular velocity of joint 3 (Example 4) ............................ 124

Fig. 2.31c. Angular acceleration of joint 3 (Example 4) ......................... 125

Fig. 2.31d. Torque of joint 3' (Example 4) .......................................... 126

Fig. 2.32a. Angular displacement of joint 3' (Example 4) ...................... 127

Fig. 2.32b. Angular velocity of joint 3' (Example 4) ….......................... 128

Fig. 2.32c. Angular acceleration of joint 3' (Example 4) ......................... 129

Fig. 2.32d. Torque of joint 2' (Example 4) ........................................... 130

Fig. 3.1. The flexible laminated hose used in the WD\&C system.......... 132

Fig. 3.2. A plane 42 element defined in ANSYS .................................. 135

Fig. 3.3. A solid 45 element defined in ANSYS ................................ 136

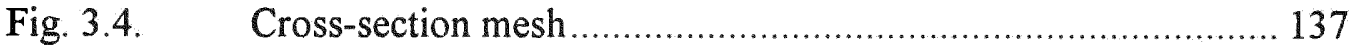

Fig. 3.5. Applied boundary conditions on the 3-D mesh....................... 139

Fig. 3.6. Deformation of the hose with 70 psi vacuum pressure ............. 141

Fig. 3.7. Stress distribution of the hose with 70 psi vacuum pressure ...... 142

Fig. 3.8. Deformation of the hose with 70 psi vacuum pressure ............ 143

Fig. 3.9. Deformation of the hose with 70 psi vacuum pressure ............. 144

Fig. 3.10. Stress distribution of the hose with 70 psi vacuum pressure ..... 145

Fig. 3.11. Stress distribution of the hose with 70 psi vacuum pressure ..... 146

Fig. 3.12. Strain distribution of the hose with 70 psi vacuum pressure ..... 147

Fig. 3.13. Strain distribution of the hose with 70 psi vacuum pressure ..... 148

Fig. 3.14. Deformation of the hose without $70 \mathrm{psi}$ vacuum pressure......... 150

Fig. 3.15. Stress distribution of the hose without 70 psi vacuum pressure 151

Fig. 3.16. Deformation of the hose without 70 psi vacuum pressure......... 152 
Fig. 3.17. Deformation of the hose without 70 psi vacuum pressure........ 153

Fig. 3.18. Stress distribution of the hose without 70 psi vacuum pressure 154

Fig. 3.19. Stress distribution of the hose without 70 psi vacuum pressure 155

Fig. 3.20. Strain distribution of the hose without 70 psi vacuum pressure 156

Fig. 3.21. Strain distribution of the hose without 70 psi vacuum pressure 157 


\section{CHAPTER 1 \\ INTRODUCTION}

The emphases of this research work are to analyze and simulate (1). the dualgripper manipulators developed to retrieve radioactive wastes from the underground storage tank with internal structural members, and (2). the flexible laminated hose used to convey the radioactive waste out of the above specified tank based on the finite element analysis (FEA).

In 1990, the DOE (Department of Energy) initiated the ER/WM (Environmental Restoration and Waste Management) program. This program joined the experience and talent among the DOE national laboratories, universities, and industrial partners. Of many critical missions specified in ER/WM program, TWR (Tank Waste Retrieval) mission is one to retrieve and remediate the radioactive wastes stored in underground storage tanks in various DOE sites. Limited access, presence of chemical, radiological and explosive hazards, and need to deliver retrieval equipment to all regions of the tank exceed the capabilities of the most conventional methods and equipment. Consequently, remotely operated devices for mobilizing and retrieving waste materials are needed. After careful review of a number of schemes for retrieving different types of materials from the underground storage tank, the use of LRMs (Long Reach Manipulators) was decided. There are two redundant TWRMS (Tank Waste Retrieval Manipulator Systems) currently operated in various DOE sites: LDUA (Light Duty Utility Arm) and MLDUA (Modified LDUA). These two redundant LRMs are known as the first generation TWRMS. To earn essential experience during the development and operation of the first generation TWRMS, they were developed and deployed in the underground storage tanks ( $25 \mathrm{ft}$ and 
$50 \mathrm{ft}$ in diameters) without any internal structures. The development and deployment of the first generation TWRMS have been successfully accomplished in the early 1997.

Continuous efforts have been made for the development of the second generation LRMs - the Remote Tool Delivery (RTD) systems. The RTD system is developed to retrieve highly radioactive wastes from the underground storage tank (diameter of 85 feet) with internal structural members approximately 10 feet apart from each other. The RTD system is a new concept that overcomes many of the limitations of the existing TWRMS. The tank's internal structural beams are used as "scaffolding" to gain access to the tank's interior, walls, ceiling and floor. The RTD system has a tethered, hydraulically powered, multi-jointed locomotor which can transverse both horizontally and vertically throughout the tank. It carries a dexterous manipulator which is used to perform localized sluicing, radiation surveying, tank wall cleaning, and inspection. The Phase 3 RTD locomotor is a four link, five jointed manipulator. The locomotor is comprised of six major components: grippers at each end, the links, the joints, the tether termination, the locomotor hydraulic system, and the video system.

In this thesis, a Phase 2 RTD system is investigated. This RTD system can be descended into underground storage tank in a riser with a minimum clearance of three feet from the bottom of the tank. Next, the Phase 3 RTD (dual-gripper) system, a superset of the Phase 2 RTD system, is modeled and analyzed. The Phase 3 RTD system is equipped with one gripper at each end to grasp the deployment mast or structural member in the underground storage tank. A vertical translation mechanism (VTM) is added to one of its links that enables the vertical motion of the RTD system inside the tank. The vertical movement can be processed with one of the grippers operating on a separate 
structural member, or the system can fold itself so that both grippers grasp the same structural member.

Kinematic models of both Phase 2 and Phase 3 RTD systems are developed in the thesis. Each joint local coordinate system and its corresponding joint parameters will be assigned based on the definition of $4 \times 4$ homogeneous C-B (Cylindrical coordinates Bryant angles) notation. The characteristic matrix of each link is derived to describe individual link shape and joint characteristics geometrically.

The dual-gripper design and inclusion of the VTM distinguish the Phase 3 RTD system from other ordinary robotic systems. This special feature provides greater dexterity and flexibility, but increases the complexity in modeling and analysis. Two operating configurations of the Phase 3 RTD system are considered and modeled, respectively. The first configuration is that gripper 1 grasps the structural member and gripper 2 is free to move in space to perform the designated task. The second configuration is that gripper 2 grasps the structural member and gripper 1 is free to move in space to perform the designated task. These two configurations typify the Phase 3 RTD system as an open-chain robotic system.

Simulations of the RTD systems, including the cases mentioned above, are completed in Tele/IGRIP simulation software package. The Tele/IGRIP simulation models can be useful for future real-time control applications.

The dual-gripper design of the Phase 3 RTD system distincts itself from other robot manipulators. It is eventually the very first of its kind based on our literature surveys for the last fifteen years. This dual-gripper system differs from the dual-arm robot manipulators. In dual-arm robots [1] the two arms work independently and they can 
function without depending upon one another. In dual-gripper robotic systems, one of the grippers has to grab a fixed structural member so that the other gripper can perform the required function. These two grippers actually interacts with each other during the operation. As a two-palm robot [2] manipulates a part three basic operations can be performed: holding or rotating the part in an equilibrium grasp if the part falls in disequilibrium, and sliding one hand or the other relative to the part. The dual-gripper robotic systems have four basic functions: grasping, lifting, sliding and rotating.

The new type of notation called C-B (Cylindrical coordinates -Bryant angles) notation [3] is used to model and analyze the dual-gripper RTD system. The method is formulated based on the homogeneous cylindrical coordinates and Byrant angles transformation matrices and thus termed as " C-B notation". It is superior to the other popular notations in modeling 3-D mechanical systems. It is noticed that D-H notation [4] only defines the "relative" joint positions, due to its joint definition, for spatial robots and mechanisms if the adjacent joint axes are not parallel or normal to each other. Moreover, identification of the joint parameters may be difficult because of the necessity of locating the common perpendiculars between lines of adjacent joint axes in the space [5]. A modified symbolism was presented by Sheth and Uicker [6] to extend the "exact " joint positions in the space. The S-U notation consists of 6 constant joint parameters $(a, b, c, \alpha, \beta, \gamma)$ and a distinct variable part that contains the same number of parameters as the degrees of freedom of the kinetic joint. In the C-B notion, characteristic matrices for 5 kinematics lower pairs: revolve, prismatic, cylindrical, helical, and spherical are derived based on homogeneous cylindrical coordinates and Bryant angles transformation 
matrices. Only five principal joint parameters $(\theta, h, r, \alpha, \beta)$ are introduced to describe the link shape and to locate the "exact" joint position in space.

As a second part of this thesis, Finite Element Analysis (FEA) of the flexible laminated hose used to convey radioactive waste materials is emphasized to learn about its structural behaviors during operation. The flexible laminated hose utilized is an AQP 2661-32 elastomer tube. This hose is reinforced with helical wire between the textile braids and AQP cover. It is generally used for suction and transfer of radioactive fluid waste. The suction hose is a bent elastic tube with six degrees-of-freedom. The hose is considered relatively fixed at one end (the outlet) and subjected to different loading conditions at the inlet. Liquid with high viscosity is sucked through the hose using a vacuum pressure.

The software package employed for FEA is ANSYS 5.2. To construct a mesh for FEA, mixed node elements are used. Material properties such as Young's Modulus and Poison's ratio of the hose are estimated. A total of 200 cases, with different $\mathrm{X}, \mathrm{Y}$ and $\mathrm{Z}$ loading conditions at the inlet, for each hose under the consideration with or without pressure, are to be analyzed. Numerical results and the ANSYS graphic outputs for each case - including the deformation, stress distribution and strain distribution - throughout the hose are depicted.

\subsection{BACKGROUND}

The present robots had their origins in both teleoperator and numerically controlled machine tool. The first manipulator known as teleoperator was developed to handle radioactive materials [7]. The teleoperator is a device which allows an operator to perform a task at a distance. In order to procure advanced aircraft whose parts were 
designed to be machined rather than riveted, the United States Air Force sponsored the development a numerically controlled (NC) milling machine [8]. The NC machine tools shapes metal automatically, based on digitally encoded cutting data. The computer controlled industrial robot was developed by Cincinnati Milacron [9], to interact with a moving conveyer whose position was sensed by a digital encoder. The computer was used to compute coordinate transformations between fixed base coordinates and the moving conveyer coordinates. As technology improved further, it became evident that robots could contribute even more. The flexible, intelligent robots are needed in advanced industry, hazardous environment and space exploration. With more manipulators being installed, the more critical needs are required for different applications in robot manipulator control.

The control of robot manipulators is based on the kinematics, especially on inverse kinematics, and dynamics. Over the past two decades, investigation on inverse kinematics for general manipulators have often been explicitly on the Jacobian pseudoinverse approach for the utilization of redundancy through local optimization of objective functions. Furthermore, most proposed methods resolve the redundancy control in joint space and are concerned with solving the inverse kinematics problem for the general robot manipulator.

Pseudoinverse control was proposed by Whitney [10]. Baillieu [11] developed different approach for local optimization by extending the Jacobian matrix to include the optimal condition. Anderson and Angeles [12] proposed a position based local optimization scheme by solving a nonlinear minimization problem with end-effector constrains. Wolovich and Elliott [13] solve the inverse kinematics problem on the 
computation of the transpose of the Jacobian. Kovecses [14] developed the dynamic and kinematic analysis of the flexible robots undergoing three - dimensional motion. Chen and Yang [15] proposed an algorithm to automatically generate the closed-form equation of the motion of a modulator robot from the kinematic graph based representation of the assembly configuration. Zhao and Shi [16] developed a concept of dual space of the Jacobian matrix. Pennock and Mattson [17] proposed the forward position problem of robot manipulating. Bicci, Prattichizzo and Melchiorri [18] proposed the theory of the force and dynamic manipulability for the general robot systems. Zhang and Yan [19] introduced the kinematic and inverse solutions for the closed chain structure in real use. Radane-cherif and Perdereau [20] proposed the iterative method to solve the inverse kinematic problem for redundant and non-redundant manipulators. Yang and Chen [21] proposed the novel kinematic calibration algorithm for reconfigurable robotic systems. Nemic [22] developed and compared the influence of unweighted and inertia weighted pseudoinverse on controlling space velocity of kinematically redundant manipulators. Caccavale and Chaiverini [23] described the application of a closed-loop forward and inverse kinematics algorithm to kinematic control of a robot manipulator. Chen and Yang [24] described the design and kinematic issues of the modular robot aimeb for assembly tasks. Merlet and Jean [25] addressed the problem finding all the solutions of the direct kinematics for every possible architecture of planar fully parallel manipulators. Tachung Yih and Kun Ji [26] proposed the generalized approach to solve the inverse kinematics problems for robots with any combination of R, P, C and S joints. Carrera and Serna [27] proposed a new and general technique for solving the inverse dynamics of general robots.Chung and Youm [28] proposed the new dynamic control method that outperforms 
existing local torque optimization techniques for Kinematically redundant manipulators. Mason and Lynch [29] describe some preliminary work on dynamic manipulation. Mayorga and Ressa [30] presented the kinematic measure of global performance for the design evaluation and optimization of robot manipulators.

Computer is an important tool in the control of robot manipulators. Early robot manipulator or teleoperators were programmed either mechanically or electrically to repeat a task once they were taught to perform. The first robot software for computer implementation was developed in 1973 at the Stanford Artificial Intelligence Laboratory [31] as an experimental research language. Later, a more advanced robot programming language based on Algol software was developed at Stanford Artificial Intelligence Laboratory and was named AL. This new software had the capability of programming assembly operations using force-sensing devices. IBM, in the meantime, was developing much more advanced robot software among which were Emily, Mi and AML. Software packages such as Tele/IGRIP and KINMAC etc, were developed for Unix systems and main frame computer to analyze and simulate the mechanical systems. 


\section{CHAPTER 2}

\section{KINEMATICS AND DYNAMICS OF DUAL-GRIPPER MANIPULATOR}

\subsection{Introduction}

Kinematically, a robot manipulator is considered an open-chain articulation of links connected in series or in parallel by prismatic, revolute, or spherical joint, etc. One end is relatively free and commonly attached with a specialized tool such as a multifingered hand or gripper, while the other end of the open-chain system is attached to a stationary base. It is apparent that the position and orientation of the robot gripper is the result of the collective effects of translation and rotation of each joint of the robotic links. Such joint motions are generated by actuators either locally activated at each joint or remotely controlled at a distance by belts, sheaves, chains, or hydraulic fluid lines.

The study of robot manipulation is concerned with the relationship between objects, and between the objects and manipulators. In this thesis the $4 \times 4$ homogeneous CB (Cylindrical coordinates - Bryant angles) notation is used for kinematic modeling and analysis of the Phase 2 RTD (Remote Tool Delivery) system and Phase 3 RTD system.

The direct kinematics applies the known joint space coordinates to find the space coordinates of the end-effector. The inverse kinematics concerns the process of designing or controlling a robot to accomplish a desired task. It determines the joint configurations from the available motions of end-effector. The direct kinematics can simply acquire a unique solution corresponding to the given joint parameters by straightforward matrix multiplication. The inverse kinematics is difficult to solve by simple computation. 


\subsection{Review of C-B Notation}

The shape geometry of the spatial binary linkage, Fig. 2.1, can be described using the transformations of cylindrical coordinates and Bryant angles; thus termed "C-B notation."

To transform the joint reference frame from $X_{i}-Y_{i}-Z_{i}$ to $X_{i+1}-Y_{i+1}-Z_{i+1}$; first, $X_{i}-Y_{i^{-}}$ $Z_{\mathrm{i}}$ is transformed to $e_{\mathrm{xi}}-\mathrm{e}_{\mathrm{yi}}-\mathrm{e}_{\mathrm{zi}}$ in the sequence $\left(\theta_{i}, \mathrm{~h}_{\mathrm{i}}, \mathrm{r}_{\mathrm{i}}\right)$ which corresponds to the cylindrical coordinate transformations; second, $\mathrm{e}_{\mathrm{xi}}-\mathrm{e}_{\mathrm{yi}}-\mathrm{e}_{\mathrm{zi}}$ is transformed to the resultant $\mathrm{X}_{\mathrm{i}+1}-\mathrm{Y}_{\mathrm{i}+1}-\mathrm{Z}_{\mathrm{i}+1}$ through Bryant angles $\left(\alpha_{i}, \beta_{i}, \gamma_{i}\right)$; with $\gamma_{i}=0^{0}$.

The joint reference frame $\left(\mathrm{X}_{\mathrm{i}}-\mathrm{Y}_{\mathrm{i}}-\mathrm{Z}_{\mathrm{i}}\right)$ and principal joint parameters $\left(\theta_{i}, h_{i}, r_{i}, \alpha_{i}, \beta_{i}\right)$ for the $i$ th joint $\mathrm{J}_{\mathrm{i}}$ are defined as follows.

\section{Reference Frame}

The local origin of the $i$ th reference frame is chosen at the physical joint center $\mathrm{J}_{\mathrm{i}}$, and:

$Z_{i}-\quad$ along the $i$ th joint axis in the designed direction.

$\mathrm{X}_{\mathrm{i}}$ - normal to $\mathrm{Z}_{\mathrm{i}}$ in the plane $\mathrm{Z}_{\mathrm{i}-} e_{X_{i-1}}$; as $\mathrm{Z}_{\mathrm{i}}$ is parallel to $e_{X_{i-1}}, \mathrm{X}_{\mathrm{i}}$ and $\mathrm{X}_{\mathrm{i}-1}$ are perpendicular to each other. Usually, $X_{i}$ is chosen in the same direction as $e_{X_{i-1}}$. $\mathrm{Y}_{\mathrm{i}}-\quad$ in the direction to form the right-handed Cartesian coordinate system.

\section{Principal Joint Parameters}

$\theta_{i}-\quad$ rotating angle of the $i$ th joint axis, measured about $Z_{i}$, as the angle between planes $\mathrm{Z}_{\mathrm{i}}-\mathrm{X}_{\mathrm{i}}$ and $\mathrm{Z}_{\mathrm{i}}-e_{X_{i}}$.

$h_{i}-\quad$ distance between joints $J_{i}$ and $J_{i+1}$, measured along $Z_{i}$.

$r_{i}-\quad$ distance between $Z_{i}$ and $J_{i+1}$. 


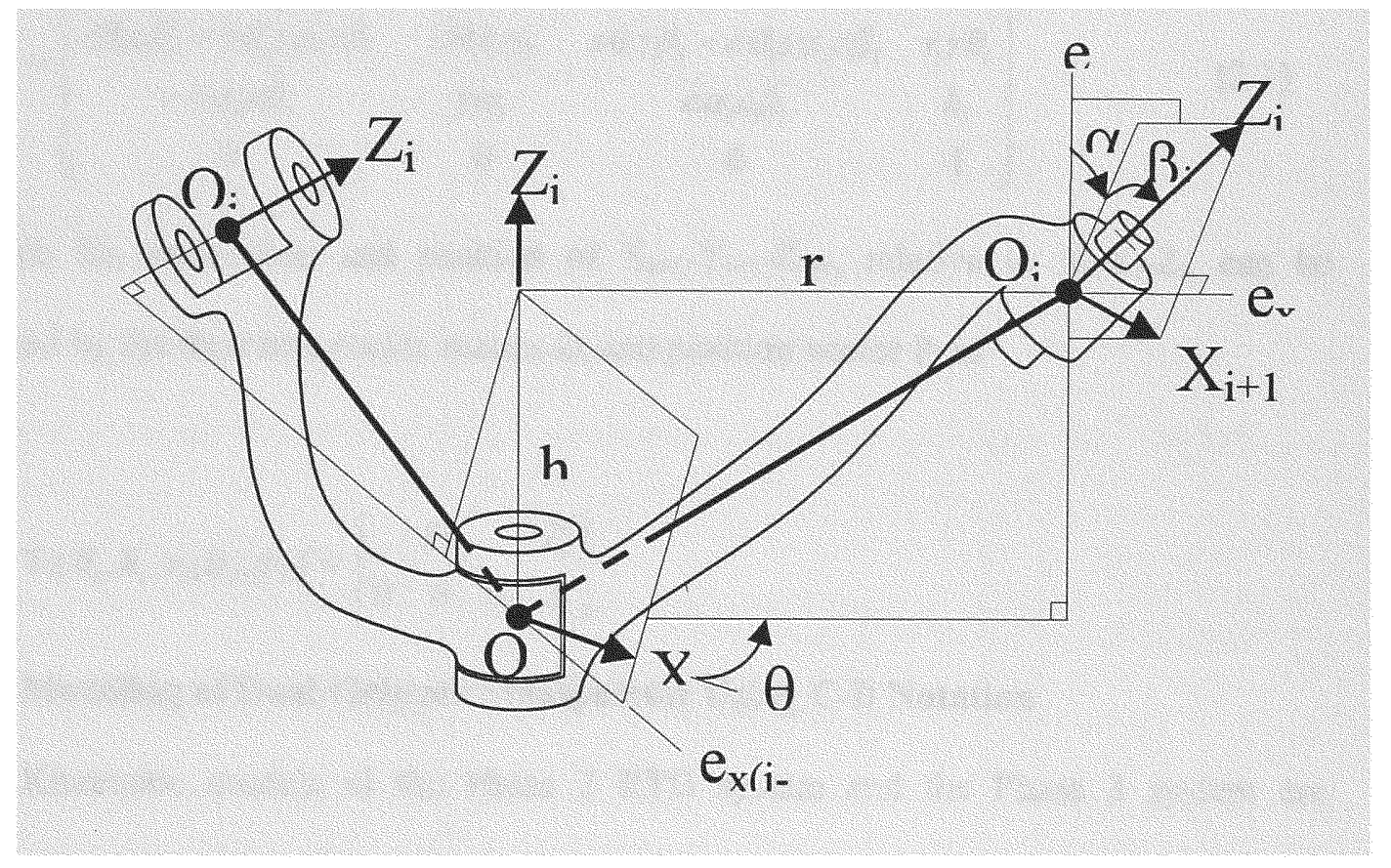

Fig. 2.1. The C-B (Cylindrical coordinate-Bryant angles) notation. 
$\alpha_{i}-\quad$ twist angle between planes $\mathrm{e}_{\mathrm{xi}}-\mathrm{e}_{\mathrm{zi}}$ and $\mathrm{e}_{\mathrm{xi}}-\mathrm{Z}_{\mathrm{i}+1}$, measured about $\mathrm{e}_{\mathrm{xi}}$. It is noticed that $X_{i+1}$ is on the plane $e_{x i}-Z_{i+1}$.

$\beta_{i}-\quad$ deviation angle between $\mathrm{e}_{\mathrm{zi}}$ and $\mathrm{Z}_{\mathrm{i}+1}$, measured from $\mathrm{e}_{\mathrm{yi}}$.

The shape matrix $\mathrm{T}_{\mathrm{i}}$ of the $i$ th link formulated by $\mathrm{C}-\mathrm{B}$ notation is, therefore,

$$
\begin{aligned}
\mathrm{T}_{\mathrm{i}} & =\mathrm{T}_{\mathrm{ci}}\left(\theta_{i}, h_{i}, r_{i}\right) \mathrm{T}_{\mathrm{bi}}\left(\alpha_{i}, \beta_{i}, 0\right) \\
& =\left[\begin{array}{cccc}
c \theta_{i} c \beta_{i}-s \theta_{i} s \alpha_{i} s \beta_{i} & -s \theta_{i} c \alpha_{i} & c \theta_{i} s \beta_{i}+s \theta_{i} s \alpha_{i} c \beta_{i} & r_{i} c \theta_{i} \\
s \theta_{i} c \beta_{i}+c \theta_{i} s \alpha_{i} s \beta_{i} & c \theta_{i} c \alpha_{i} & s \theta_{i} s \beta_{i}-c \theta_{i} s \alpha_{i} c \beta_{i} & r_{i} s \theta_{i} \\
-c \alpha_{i} s \beta_{i} & s \alpha_{i} & c \alpha_{i} c \beta_{i} & h_{i} \\
0 & 0 & 0 & 1
\end{array}\right]
\end{aligned}
$$

in which the orientation and position of $X_{i+1}-Y_{i+1}-Z_{i+1}$, relative to $X_{i}-Y_{i}-Z_{i}$, can be expressed by the direction cosine matrix $D_{i}$ and position vector $P_{i}$ as

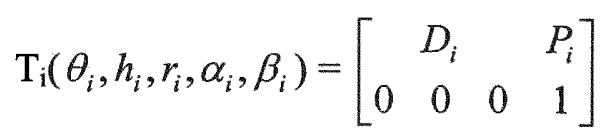

\subsection{Modeling of Dual-Gripper Manipulator Using C-B Notation}

Kinematic models of the Phase 2 RTD system and the Phase 3 system are developed in this section. Fixed frame reference and each joint axis are assigned based on the definition of $4 \times 4$ homogeneous $\mathrm{C}-\mathrm{B}$ notation. The characteristic matrix $\mathrm{T}_{\mathrm{i}}$ of the $i$ th link is derived to geometrically describe individual joint characteristics and link shape.

\subsubsection{The Phase 2 RTD System}

As shown in Fig. 2.2, a fixed frame of reference is designated at the center of rotek bearing (elevation 101') which is 3 feet above the ground surface. The RTD system 


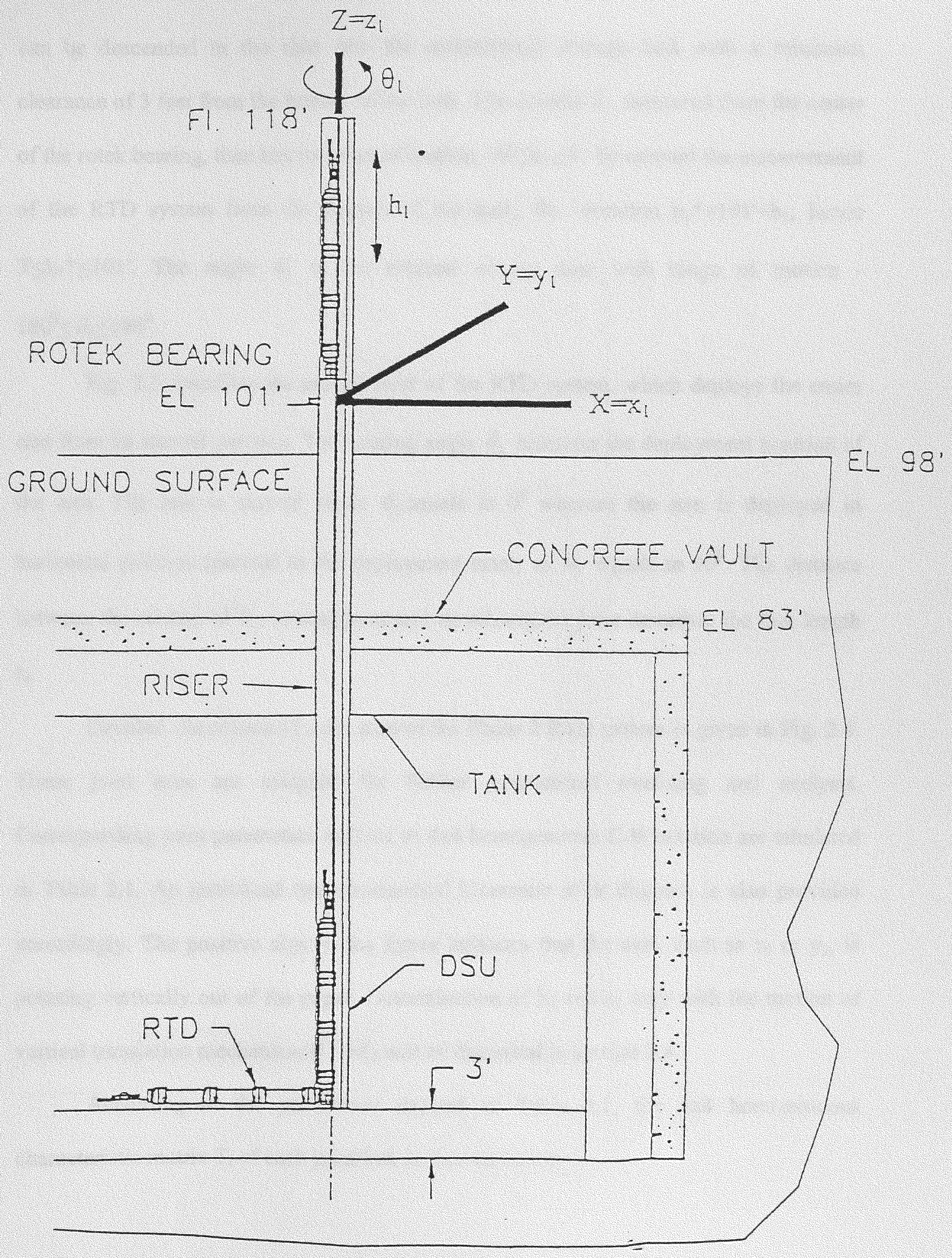

Fig. 2.2 The fixed frame of reference for Phase 2 RTD system 
can be descended in the riser into the underground storage tank with a minimum clearance of 3 feet from the bottom of the tank. The descent $h_{1}$, measured from the center of the rotek bearing, then has its range of motion $-98^{\prime} \leq h_{1} \leq 0^{\prime}$. To convert the measurement of the RTD system from the bottom of the tank, the elevation $h_{1}^{*}=101^{\prime}+h_{1}$; hence $3^{\prime} \leq \mathrm{h}_{1}{ }^{*} \leq 101^{\prime}$. The angle $\theta_{1}$ is the rotation of the riser with range of motion $180^{\circ} \leq \theta_{1} \leq 180^{\circ}$

Fig. 2.3 describes the second joint of the RTD system, which deploys the entire arm from its stowed position. The rotating angle $\theta_{2}$ indicates the deployment position of the arm. The arm is stowed while $\theta_{2}$ equals to $0^{0}$ whereas the arm is deployed in horizontal position (normal to the deployment mast) as $\theta_{2}$ equals to $90^{\circ}$. The distance between the centers of the second joint and its subsequent joint describes the link length $\mathrm{r}_{2}$.

Detailed illustration of joint axes of the Phase 2 RTD system is given in Fig. 2.4. These joint axes are assigned for further geometrical modeling and analysis. Corresponding joint parameters defined in $4 \times 4$ homogeneous C-B notation are tabulated in Table 2.1. An initialized two-dimensional kinematic stick diagram is also provided accordingly. The positive sign in the figure indicates that the axis, such as $z_{2}$ or $y_{3}$, is pointing vertically out of the paper. Determination of $h_{5}$ and $r_{5}$ vary with the motion of vertical translation mechanism (VTM) will be discussed in section 2.4 .

According to the parameters defined in Table 2.1, the $4 \times 4$ homogeneous characteristic matrix $T_{i}$ of each joint/link is derived below. 


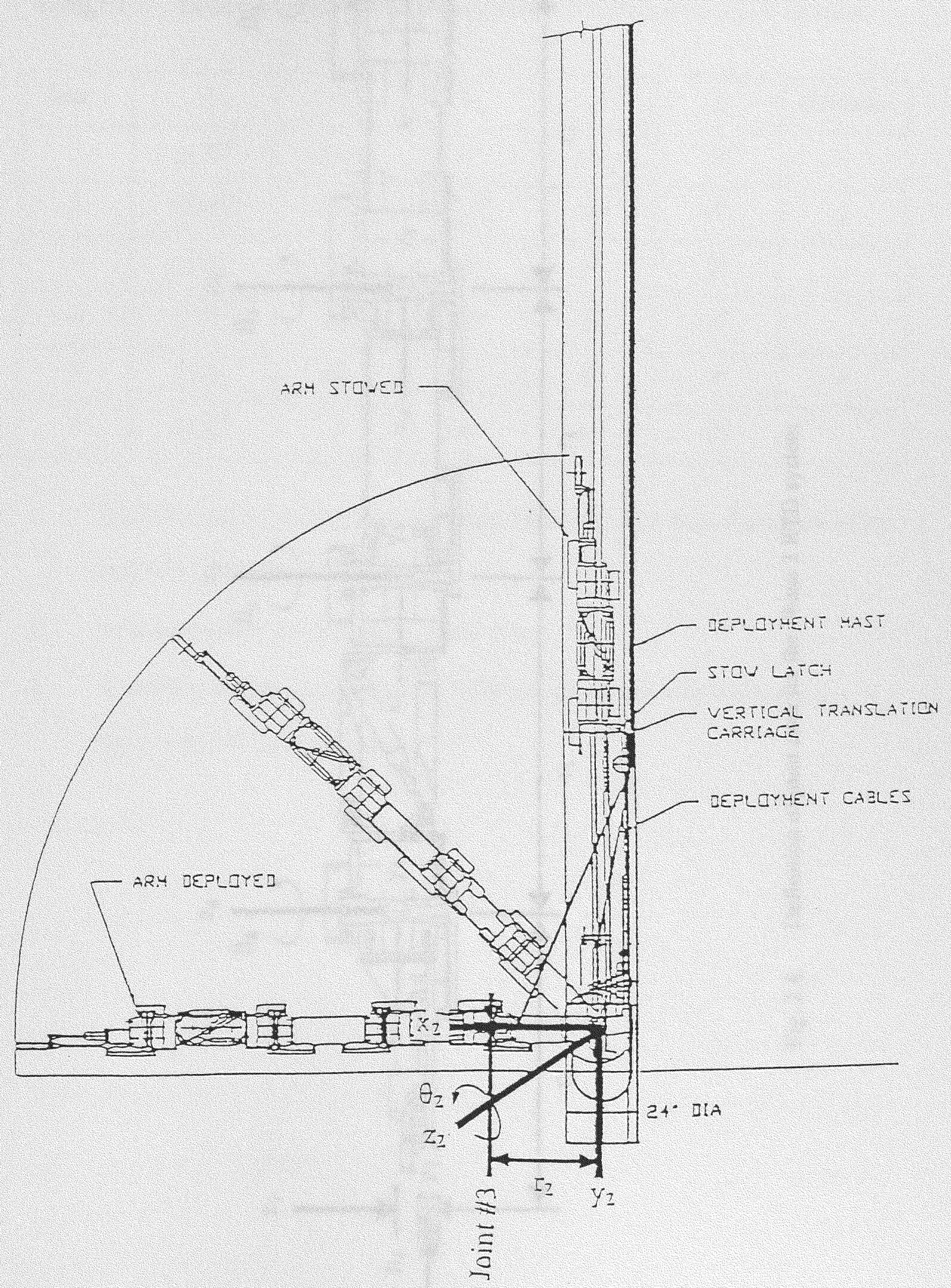

Fig. 2.3 Definition of Joint 勒, Phase 2 RTD system, for arm deployment 


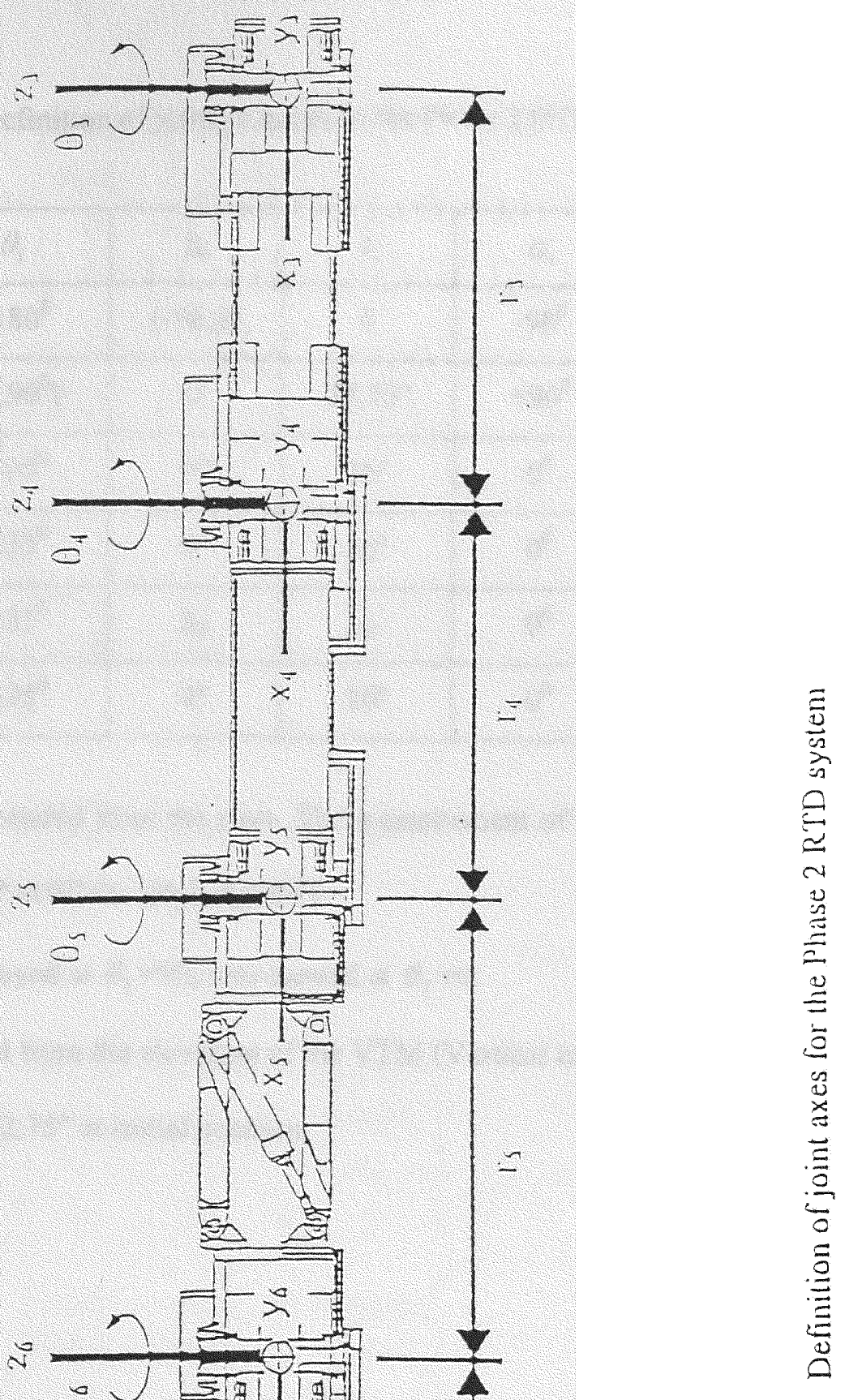

5

E 
Table 2.1 Definition of joint parameters for Phase 2 RTD system in C-B notation

\begin{tabular}{|c|c|c|c|c|c|c|}
\hline Joint 1 & $\theta_{i}$ & $\mathrm{~h}_{\mathrm{i}}$ & $\mathrm{r}_{\mathrm{i}}$ & $\alpha_{i}$ & $\beta_{i}$ & Remarks \\
\hline 1 & $\pm 180^{\circ}$ & $(-98,0)$ & 0 & $-90^{0}$ & $0^{0}$ & 1 \\
\hline 2 & $\left(0^{0}, 90^{\circ}\right)$ & $0^{\prime}$ & $37.75^{\prime \prime}$ & $+90^{0}$ & $0^{0}$ & 2 \\
\hline 3 & $\pm 135^{\circ}$ & $0^{\prime \prime}$ & $36^{\prime \prime}$ & $0^{0}$ & $0^{0}$ & - \\
\hline 4 & $\pm 135^{0}$ & $0^{\prime \prime}$ & $36^{\prime \prime}$ & $0^{0}$ & $0^{0}$ & - \\
\hline 5 & $\pm 135^{\circ}$ & $\mathrm{h}_{5}$ & $\mathrm{r}_{5}$ & $0^{0}$ & $0^{0}$ & 3 \\
\hline 6 & $\pm 135^{\circ}$ & $4^{\prime \prime}$ & $36^{\prime \prime}$ & $0^{0}$ & $0^{0}$ & - \\
\hline
\end{tabular}

1. RTD descended from the riser. The measurement of RTD from the bottom of the $\operatorname{tank}$ is $\mathrm{h}_{1}{ }^{*}=101^{\prime}+\mathrm{h}_{1}\left(3^{\prime} \leq \mathrm{h}_{1}{ }^{*} \leq 101^{\prime}\right)$.

2. Arm deployed at $\theta_{2}=90$; arm stowed at $\theta_{2}=0$.

3. $h_{5}$ resulted from the elevation of the VTM (Vertical translation Mechanism). $r_{5}$ is equal to $42.75^{\prime \prime}$ at initial position. 


$$
\begin{aligned}
& \mathrm{T}_{1}=\left[\begin{array}{cccc}
\cos \theta_{1} & 0 & -\sin \theta_{1} & 0 \\
\sin \theta_{1} & 0 & \cos \theta_{1} & 0 \\
0 & -1 & 0 & h_{1} \\
0 & 0 & 0 & 1
\end{array}\right] \\
& \mathrm{T}_{2}=\left[\begin{array}{cccc}
\cos \theta_{2} & 0 & \sin \theta_{2} & r_{2} \cos \theta_{2} \\
\sin \theta_{2} & 0 & -\cos \theta_{2} & r_{2} \sin \theta_{2} \\
0 & 1 & 0 & 0 \\
0 & 0 & 0 & 1
\end{array}\right] \\
& \mathrm{T}_{3}=\left[\begin{array}{cccc}
\cos \theta_{3} & -\sin \theta_{3} & 0 & r_{3} \cos \theta_{3} \\
\sin \theta_{3} & \cos \theta_{3} & 0 & r_{3} \sin \theta_{3} \\
0 & 0 & 1 & 0 \\
0 & 0 & 0 & 1
\end{array}\right] \\
& \mathrm{T}_{4}=\left[\begin{array}{cccc}
\cos \theta_{4} & -\sin \theta_{4} & 0 & r_{4} \cos \theta_{4} \\
\sin \theta_{4} & \cos \theta_{4} & 0 & r_{4} \sin \theta_{4} \\
0 & 0 & 1 & 0 \\
0 & 0 & 0 & 1
\end{array}\right] \\
& \mathrm{T}_{5}=\left[\begin{array}{cccc}
\cos \theta_{5} & -\sin \theta_{5} & 0 & r_{5} \cos \theta_{5} \\
\sin \theta_{5} & \cos \theta_{5} & 0 & r_{5} \sin \theta_{5} \\
0 & 0 & 1 & h_{5} \\
0 & 0 & 0 & 1
\end{array}\right] \\
& \mathrm{T}_{6}=\left[\begin{array}{cccc}
\cos \theta_{6} & -\sin \theta_{6} & 0 & r_{6} \cos \theta_{6} \\
\sin \theta_{6} & \cos \theta_{6} & 0 & r_{6} \sin \theta_{6} \\
0 & 0 & 1 & h_{6} \\
0 & 0 & 0 & 1
\end{array}\right]
\end{aligned}
$$

The direction (orientation) of each joint axis and the location (position) of each joint center relative to the universal frame of reference $X-Y-Z\left(x_{1}-y_{1}-z_{1}\right)$ can be determined by

$$
\mathrm{H}=\prod_{i=1}^{6} \mathrm{~T}_{\mathrm{i}}
$$


where $T_{i}$ is the characteristic matrix of the $i$ th joint and $H$ is the resultant $4 x 4$ homogeneous matrix that contains the numerical data of orientation and position of the axis of gripper center $\mathrm{x}_{7}-\mathrm{y}_{7}-\mathrm{Z}_{7}$.

\subsubsection{The Phase 3 RTD System}

The Phase 3 RTD, Fig. 2.5, system is a superset of the Phase 2 RTD System. Each end of the system is equipped with a gripper to grasp the deployment mast or structural member in the tank. A vertical translation mechanism (VTM) is added which enables vertical motion of the system inside the tank. Two vertical translation modes are possible: 1) the vertical movement can be processed and performed with each gripper operating on a separate structural member, and 2) the system can fold so that both grippers grasp the same structural member. The first mode typifies the open-chain robotic system and will be the emphasis of this thesis. The second mode exemplifies the standard closed-chain mechanism and will be studied as a separate topic.

For the first mode of open-chain robotic system, two possible configurations are investigated. They are 1) gripper 1 grasps the deployment mast or structural member in the tank, and 2) gripper 2 grasps the deployment mast or structural member in the tank.

The dual-gripper design and the inclusion of VTM distinguish the Phase 3 RTD system from other ordinary robotic systems. These special features provide greater dexterity and flexibility; yet, increases the complexity in modeling and analysis.

As shown in Fig. 2.5, local frame of reference for each joint is designated at its joint center. Due to the symmetry of the structure, gripper joint 2 is redefined as joint 1 , with link length $r_{1}^{\prime}$. The VTM is located between joints 2 and 3 . The middle section of the system is defined between joints 3 and $3^{\prime}$ with link length $r_{3}$ equals $r^{\prime}{ }_{3}$. Since the VTM is 


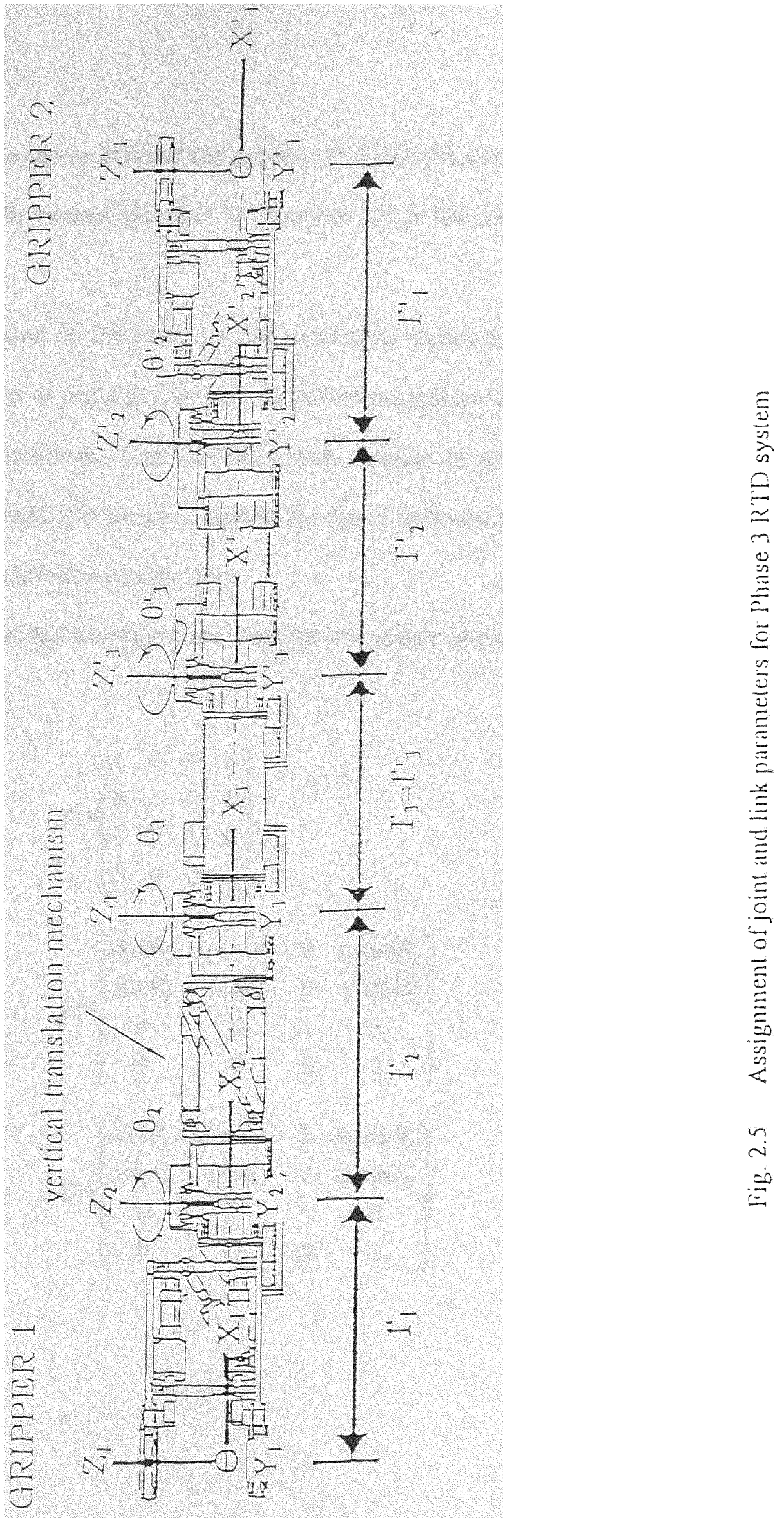


able to elevate or descend the system vertically, the distance between joints 2 and $3\left(r_{2}\right)$ varies with vertical elevation $h_{2}$. However, other link lengths will remain constant at all times.

Based on the joint and link parameters assigned in Fig. 2.5, their corresponding magnitudes or variables defined in $4 \times 4$ homogeneous C-B notation are listed in Table 2.2. A two-dimensional kinematic stick diagram is provided to specify its initialized configuration. The negative sign in the figure indicates that the joint axis, such as $\mathrm{y}_{2}$, is pointing vertically into the page.

The $4 \times 4$ homogeneous characteristic matrix of each joint/link then can be derived as follows.

$$
\begin{aligned}
\mathrm{T}_{1} & =\left[\begin{array}{llll}
1 & 0 & 0 & r_{1} \\
0 & 1 & 0 & 0 \\
0 & 0 & 1 & 0 \\
0 & 0 & 0 & 1
\end{array}\right] \\
\mathrm{T}_{2} & =\left[\begin{array}{cccc}
\cos \theta_{2} & -\sin \theta_{2} & 0 & r_{2} \cos \theta_{2} \\
\sin \theta_{2} & \cos \theta_{2} & 0 & r_{2} \sin \theta_{2} \\
0 & 0 & 1 & h_{2} \\
0 & 0 & 0 & 1
\end{array}\right] \\
\mathrm{T}_{3} & =\left[\begin{array}{cccc}
\cos \theta_{3} & -\sin \theta_{3} & 0 & r_{3} \cos \theta_{3} \\
\sin \theta_{3} & \cos \theta_{3} & 0 & r_{3} \sin \theta_{3} \\
0 & 0 & 1 & 0 \\
0 & 0 & 0 & 1
\end{array}\right]
\end{aligned}
$$


Table 2.2 Definition of joint parameters for case 1 study of the Phase 3 RTD system

\begin{tabular}{|c|c|c|c|c|c|}
\hline Joint 1 & $\theta_{i}$ & $\mathrm{~h}_{\mathrm{i}}$ & $\mathrm{r}_{\mathrm{i}}$ & $\alpha_{i}$ & $\beta_{i}$ \\
\hline 1 & $0^{0}$ & $0^{\prime \prime}$ & $36^{\prime \prime}$ & $0^{0}$ & $0^{0}$ \\
\hline 2 & $\pm 135^{\circ}$ & $\pm 8^{\prime \prime}$ & $\left(40.931^{\prime \prime}, 42.75^{\prime \prime}\right)^{*}$ & $0^{0}$ & $0^{0}$ \\
\hline 3 & $\pm 135^{\circ}$ & $0^{\prime \prime}$ & $36^{\prime \prime}$ & $0^{0}$ & $0^{0}$ \\
\hline $3^{\prime}$ & $\pm 135^{\circ}$ & $0^{\prime \prime}$ & $36^{\prime \prime}$ & $0^{0}$ & $0^{0}$ \\
\hline $2^{\prime}$ & $\pm 135^{\circ}$ & $0^{\prime \prime}$ & $36^{\prime \prime}$ & $0^{0}$ & $0^{0}$ \\
\hline $1^{\prime}$ & $0^{0}$ & $0^{\prime \prime}$ & $0^{\prime \prime}$ & $0^{0}$ & $0^{0}$ \\
\hline
\end{tabular}

$r_{2}$ is equal to $42.75^{11}$ at initial position 


$$
\begin{aligned}
& \mathrm{T}_{4}=\left[\begin{array}{cccc}
\cos \theta_{3}^{\prime} & -\sin \theta_{3}^{\prime} & 0 & r_{2}^{\prime} \cos \theta_{3}{ }_{3} \\
\sin \theta_{3}^{\prime} & \cos \theta_{3}^{\prime} & 0 & r_{2}^{\prime} \sin \theta_{3} \\
0 & 0 & 1 & 0 \\
0 & 0 & 0 & 1
\end{array}\right] \\
& \mathrm{T}_{5}=\left[\begin{array}{cccc}
\cos \theta_{2}^{\prime} & -\sin \theta_{2}^{\prime} & 0 & r_{1}^{\prime} \cos \theta_{2} \\
\sin \theta_{2}^{\prime} & \cos \theta_{2}^{\prime} & 0 & r_{1}^{\prime} \sin \theta_{2}^{\prime} \\
0 & 0 & 1 & 0 \\
0 & 0 & 0 & 1
\end{array}\right] \\
& \mathrm{T}_{6}=\left[\begin{array}{llll}
1 & 0 & 0 & 0 \\
0 & 1 & 0 & 0 \\
0 & 0 & 1 & 0 \\
0 & 0 & 0 & 1
\end{array}\right]
\end{aligned}
$$

The direction (orientation) of each joint axis and location (position) of each joint center relative to $\mathrm{X}_{1}-\mathrm{Y}_{1}-\mathrm{Z}_{1}$ can be determined by

$$
\mathrm{H}=\prod_{i=1}^{6} \mathrm{~T}_{\mathrm{i}}
$$

where $T_{i}$ is the characteristic matrix derived above, and $H$ is the resultant $4 x 4$ homogeneous matrix that specifies the orientation and position of gripper 2 relative to gripper 1.

Subsequently, the two possible configurations of the phase 3 RTD system as an open-chain system are studied.

a). Case 1 - Gripper 1 grasps the deployment mast or structural member in the tank

The first possible operating configuration for the Phase 3 RTD system is shown Fig. 2.6, where gripper 1 grasps the deployment mast or structural member in the tank whereas gripper 2 is free to move in space. This configuration typifies an open-chain robotic system. The joint and link parameters defined in Table 2.2 are applicable to this 




Fig. 2.6 Case 1 - Gripper 1 grasps the deployment mast or structural member in the tack 
Table 2.3 Definition of joint parameters for case 2 study of the Phase 3 RTD system

\begin{tabular}{|c|c|c|c|c|c|}
\hline Joint 1 & $\theta_{i}$ & $\mathrm{~h}_{\mathrm{i}}$ & $\mathrm{r}_{\mathrm{i}}$ & $\alpha_{i}$ & $\beta_{i}$ \\
\hline $1^{\prime}$ & $0^{0}$ & $0^{\prime \prime}$ & $-36^{\prime \prime}$ & $0^{0}$ & $0^{0}$ \\
\hline $2^{\prime}$ & $\pm 135^{0}$ & $0^{\prime \prime}$ & $-36^{\prime \prime}$ & $0^{0}$ & $0^{0}$ \\
\hline $3^{\prime}$ & $\pm 135^{\circ}$ & $0^{\prime \prime}$ & $-36^{\prime \prime}$ & $0^{0}$ & $0^{0}$ \\
\hline 3 & $\pm 135^{0}$ & $\pm 8^{\prime \prime}$ & $\left(-42.75^{\prime \prime},-40.931^{\prime \prime}\right)$ & $0^{0}$ & $0^{0}$ \\
\hline 2 & $\pm 135^{0}$ & $0^{\prime \prime}$ & $-36^{\prime \prime}$ & $0^{0}$ & $0^{0}$ \\
\hline 1 & $0^{0}$ & $0^{\prime \prime}$ & $0^{\prime \prime}$ & $0^{0}$ & $0^{0}$ \\
\hline
\end{tabular}

$r_{2}$ is equal to $42.75^{\prime \prime}$ at the initial position. 
case, and the characteristic matrices derived in equations (2.4a) through (2.4f) and 2.5 are also valid for this application.

b). $\quad$ Case 2 - Gripper 2 grasps the deployment mast or structural member in the tank

It can be appropriate, in some occasions, that gripper 2 grasps the deployment mast or structural member in the tank while gripper 1 performs the necessary task. Configuration of the Phase 3 RTD system operated in this case is depicted in Fig. 2.7. The joint and link variables, also referring to Fig. 2.5, defined in $4 \times 4$ homogeneous C-B notation are listed in Table 2.3.

The $4 \times 4$ homogeneous matrix of each joint/link are derived as

$$
\begin{aligned}
& \mathrm{T}_{1}=\left[\begin{array}{cccc}
1 & 0 & 0 & -r_{1}^{\prime} \\
0 & 1 & 0 & 0 \\
0 & 0 & 1 & 0 \\
0 & 0 & 0 & 1
\end{array}\right] \\
& \mathrm{T}_{2}=\left[\begin{array}{cccc}
\cos \theta_{2}^{\prime} & -\sin \theta_{2}^{\prime} & 0 & -r_{2}^{\prime} \cos \theta_{2}^{\prime} \\
\sin \theta_{2}^{\prime} & \cos \theta_{2}^{\prime} & 0 & -r_{2}^{\prime} \sin \theta_{2}^{\prime} \\
0 & 0 & 1 & 0 \\
0 & 0 & 0 & 1
\end{array}\right] \\
& \mathrm{T}_{3}=\left[\begin{array}{cccc}
\cos \theta_{3}^{\prime} & -\sin \theta_{3}^{\prime} & 0 & -r_{3} \cos \theta_{3}^{\prime} \\
\sin \theta_{3}^{\prime} & \cos \theta_{3}^{\prime} & 0 & -r_{3} \sin \theta_{3}^{\prime} \\
0 & 0 & 1 & 0 \\
0 & 0 & 0 & 1
\end{array}\right] \\
& \mathrm{T}_{4}=\left[\begin{array}{cccc}
\cos \theta_{3} & -\sin \theta_{3} & 0 & -r_{2} \cos \theta_{3} \\
\sin \theta_{3} & \cos \theta_{3} & 0 & -r_{2} \sin \theta_{3} \\
0 & 0 & 1 & h_{2} \\
0 & 0 & 0 & 1
\end{array}\right]
\end{aligned}
$$




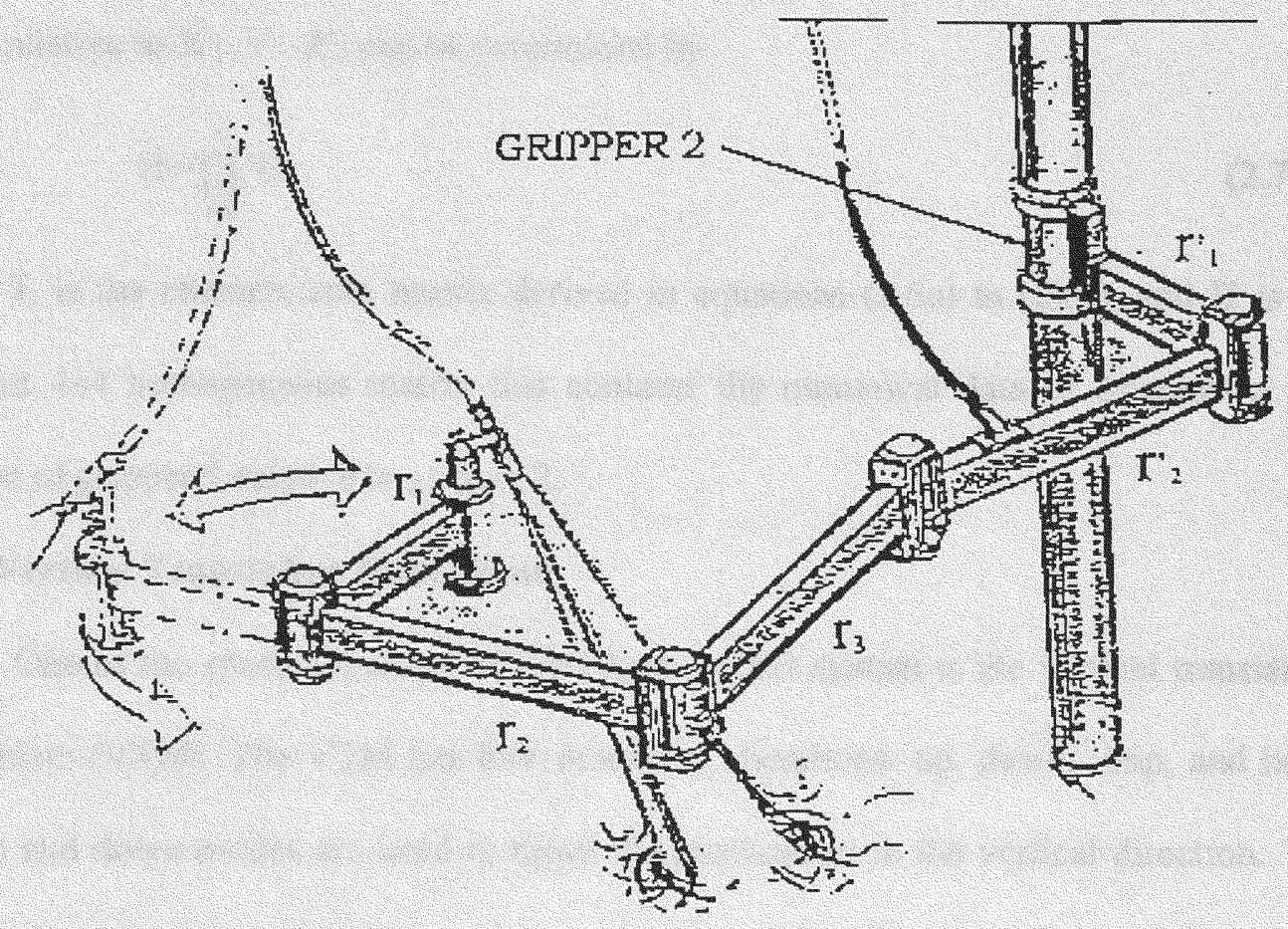

Fig. 2.7 Case 2 - Gripper 2 grasps the deployment mast or structural member in the tank 


$$
\begin{aligned}
& \mathrm{T}_{5}=\left[\begin{array}{cccc}
\cos \theta_{2} & -\sin \theta_{2} & 0 & r_{1} \cos \theta_{2} \\
\sin \theta_{2} & \cos \theta_{2} & 0 & r_{1} \sin \theta_{2} \\
0 & 0 & 1 & 0 \\
0 & 0 & 0 & 1
\end{array}\right] \\
& \mathrm{T}_{6}=\left[\begin{array}{llll}
1 & 0 & 0 & 0 \\
0 & 1 & 0 & 0 \\
0 & 0 & 1 & 0 \\
0 & 0 & 0 & 1
\end{array}\right]
\end{aligned}
$$

The direction (orientation) of each joint axis and location (position) of each joint center relative to $X_{1}{ }_{1}-Y_{1}^{\prime}{ }_{1}-Z_{1}^{\prime}$ can be determined by

$$
\mathrm{H}=\prod_{i=1}^{6} \mathrm{~T}_{\mathrm{i}}
$$

where $T_{i}$ is the characteristic matrix derived in equations (2.6a) to (2.6f), and $H$ is the resultant $4 \times 4$ homogeneous matrix that contains the numerical data of orientation and position of gripper 1 relative to gripper 2 .

\subsection{Vertical Translation Mechanism}

One of the special features of the Phase 3 RTD system is the vertical translation mechanism (VTM). The VTM has four modes of operations: up, down, limp, and hold. The up and down modes are used to move the mechanism in the vertical direction. The hold mode is to hold the vertical motion at a fixed position. The fail-safe condition of the VTM is the limp mode. The vertical translation mechanism, a parallelogram four-bar mechanism, is associated with link 2 in the Phase 3 RTD system as shown in Fig. 2.8. The VTM is located at a distance of $12.125^{\prime \prime}$ from both $Z_{2}$ and $Z_{3}$. Since the VTM elevates or descents the system vertically, both vertical $\left(\mathrm{h}_{2}\right)$ and horizontal $\left(\mathrm{r}_{2}\right)$ distances 


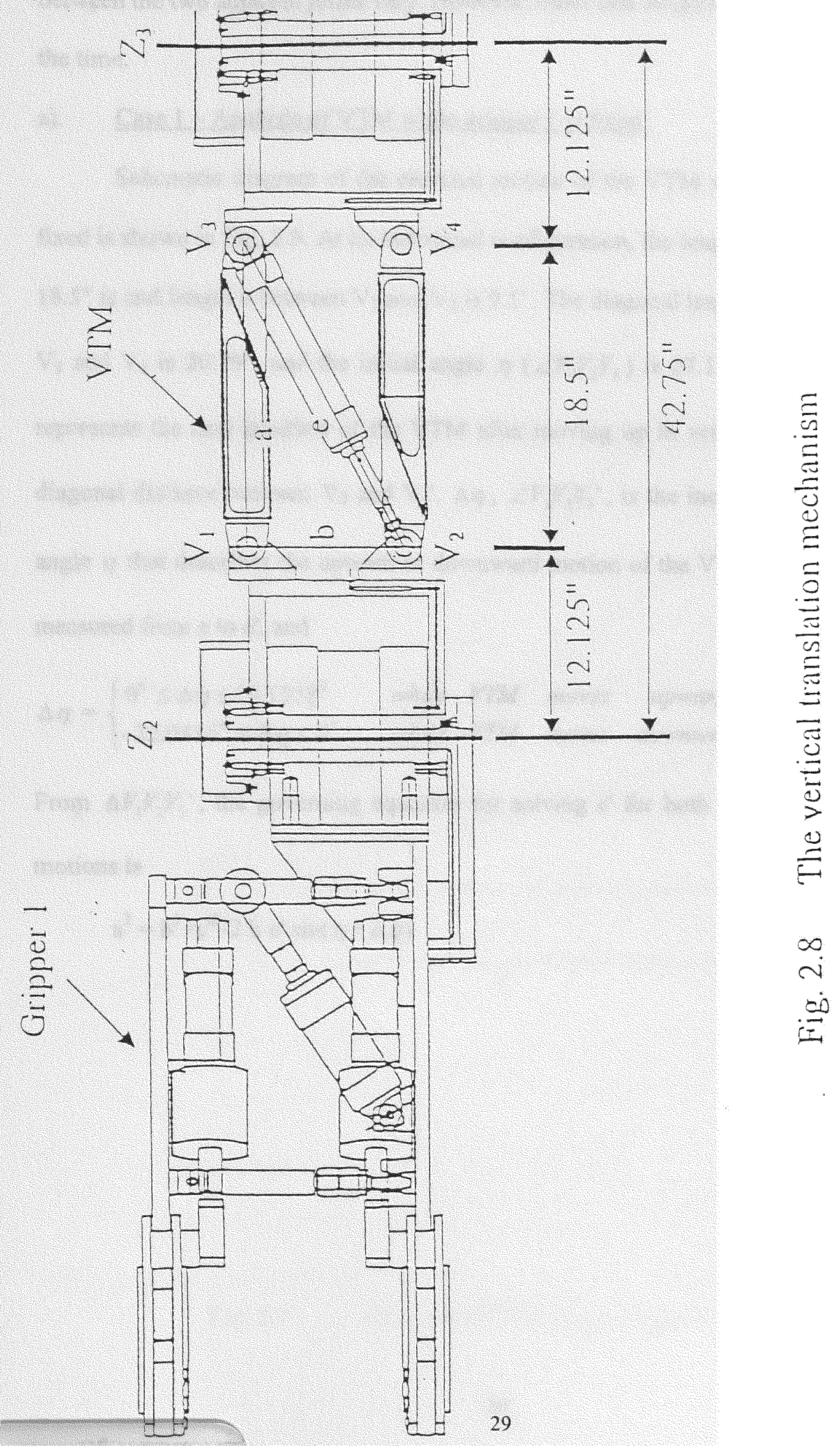


between the two adjacent joints vary. However, other link lengths will remain constant all the time.

a). Case 1 - Analysis of VTM while gripper 1 is fixed.

Schematic diagram of the elevated motion of the VTM with gripper 1 relatively fixed is shown in Fig. 2.9. At its initialized configuration, the length a between $V_{1}$ and $V_{3}$ $18.5^{\prime \prime}$ is and length $b$ between $V_{1}$ and $V_{2}$ is $9.5^{\prime \prime}$. The diagonal length e measured between $\mathrm{V}_{2}$ and $\mathrm{V}_{3}$ is $20.79^{\prime \prime}$, and the initial angle $\eta\left(\angle V_{3} V_{2} V_{4}\right)$ is $27.18^{0}$. Linkage $\mathrm{V}_{1} \mathrm{~V}_{3}{ }^{\prime} \mathrm{V}_{4}{ }^{\prime} \mathrm{V}_{2}$ represents the new position of the VTM after moving up in vertical direction. $e^{\prime}$ is the diagonal distance between $\mathrm{V}_{2}$ and $\mathrm{V}_{3}^{\prime}, \Delta \eta, \angle V_{3} V_{2} V_{3}^{\prime}$, is the increment or decrement of angle $\eta$ that describes the upward or downward motion of the VTM, respectively. $\Delta \eta$ is measured from e to e', and

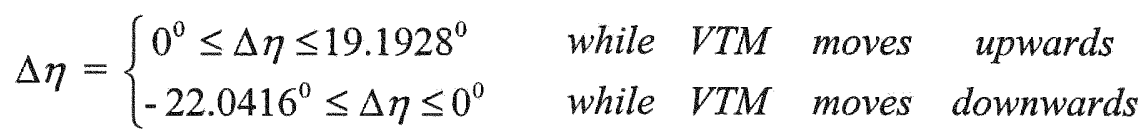

From $\Delta V_{1} V_{2} V_{3}^{\prime}$, the governing equation for solving $\mathrm{e}^{\prime}$ for both upward and downward motions is

$$
\mathrm{a}^{2}=\mathrm{b}^{2}+\mathrm{e}^{\prime 2}-2 \mathrm{~b} \mathrm{e}^{\prime} \sin (\eta+\Delta \eta)
$$




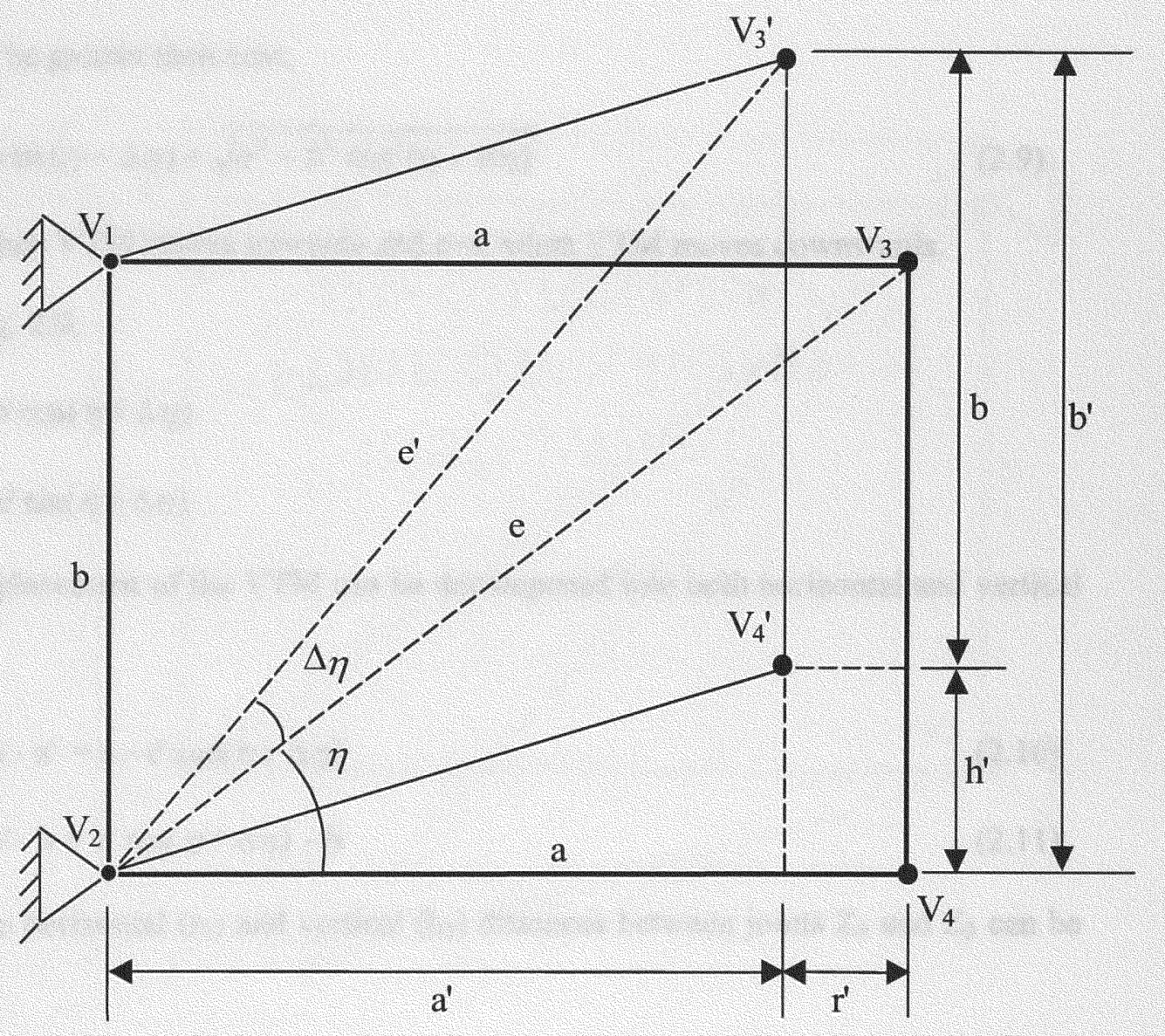

Fig. 2.9 Analysis of VTM while gripper 1 is fixed 
Rearranging the above equation,

$$
\mathrm{e}^{\prime 2}+\mathrm{e}^{\mathrm{t}}[-2 \mathrm{~b} \sin (\eta+\Delta \eta)]+\left(\mathrm{b}^{2}-\mathrm{a}^{2}\right)=0
$$

Solving the quadratic equation for e', yields,

$$
\mathrm{e}^{\prime}=\frac{2 b \sin (\eta+\Delta \eta) \pm \sqrt{4 b^{2} \sin ^{2}(\eta+\Delta \eta)-4 b^{2}+4 a^{2}}}{2}
$$

Since e' must be greater than zero,

$$
\mathrm{e}^{\prime}=b \sin (\eta+\Delta \eta)+\sqrt{a^{2}-b^{2} \cos ^{2}(\eta+\Delta \eta)}
$$

where $\mathrm{e}^{\prime}>\mathrm{e}$ when VTM moves upwards and $\mathrm{e}^{\prime}<\mathrm{e}$ when VTM moves downwards.

Also from Fig. 2.9,

$$
\begin{aligned}
& \mathrm{a}^{\prime}=\mathrm{e}^{\prime} \cos (\eta+\Delta \eta) \\
& \mathrm{b}^{\prime}=\mathrm{e}^{\prime} \sin (\eta+\Delta \eta)
\end{aligned}
$$

The local displacement of the VTM can be decomposed into both horizontal and vertical directions as

$$
\begin{aligned}
& \mathrm{r}^{\prime}=\mathrm{a}-\mathrm{a}^{\prime}=\mathrm{a}-\mathrm{e}^{\prime} \cos (\eta+\Delta \eta) \\
& \mathrm{h}^{\prime}=\mathrm{b}^{\prime}-\mathrm{b}=\mathrm{e}^{\prime} \sin (\eta+\Delta \eta)-\mathrm{b}
\end{aligned}
$$

Therefore, the horizontal $\left(r_{2}\right)$ and vertical $\left(h_{2}\right)$ distances between joints $Z_{2}$ and $Z_{3}$ can be calculated as

$$
\begin{aligned}
& r_{2}=a^{\prime}+24.25^{\prime \prime}=42.75^{\prime \prime}-r^{\prime} \\
& h_{2}=h^{\prime}
\end{aligned}
$$

b). $\quad$ Case 2 - Analysis of VTM while gripper 2 is fixed.

With reference to Fig. 2.10, the descendent motion of the VTM with gripper 2 relatively fixed is depicted. At its initialized configuration, the lengths $a, b$, and e are 


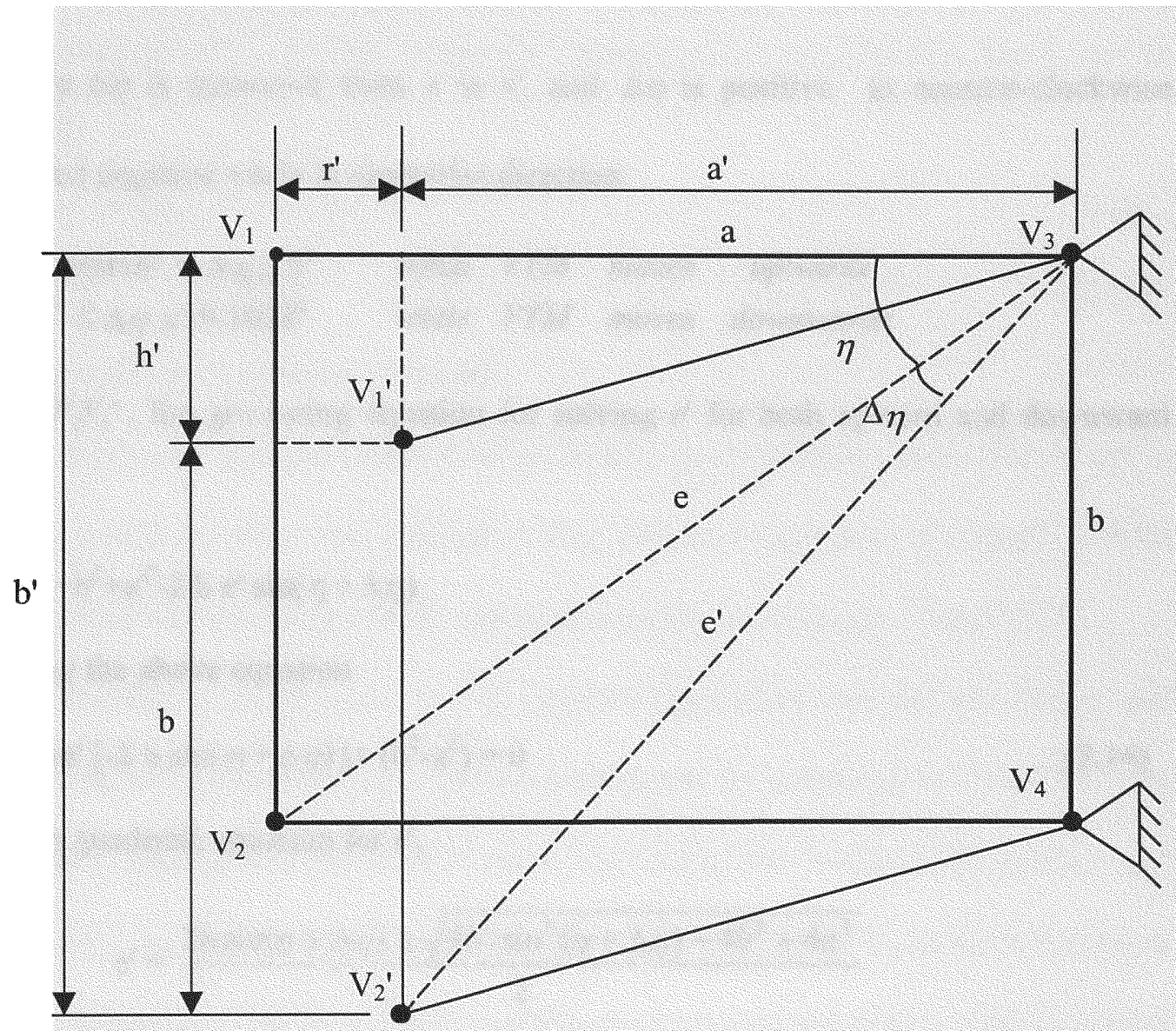

Fig. 2.10 Analysis of VTM while gripper 2 is fixed 
18.5", 9.5", and 20.79", respectively. The initial angle $\eta\left(\angle V_{1} V_{3} V_{2}\right)$ is $27.18^{\circ}$. Linkage $\mathrm{V}_{3} \mathrm{~V}_{1}{ }^{\prime} \mathrm{V}_{2}{ }^{\prime} \mathrm{V}_{4}$ indicates the new position of the VTM after moving down in vertical direction. $\mathrm{e}^{\prime}$ is the distance between $\mathrm{V}_{2}$ and $\mathrm{V}_{3}{ }^{\prime} . \Delta \eta, \angle V_{2} V_{3} V_{2}^{\prime}$, is the increment or decrement of $\eta$ to describe the upward or downward motion of the VTM, respectively. $\Delta \eta$ is measured from e to $\mathrm{e}^{\prime}$, and $\Delta \eta$ is positive in counter-clockwise direction and negative while in clockwise direction.

$$
\Delta \eta=\left\{\begin{aligned}
-22.0416^{\circ} \leq \Delta \eta \leq 0^{\circ} & \text { while } V T M \text { moves upwards } \\
0^{0} \leq \Delta \eta \leq 19.1928^{\circ} & \text { while VTM moves downwards }
\end{aligned}\right.
$$

From $\Delta V_{3} V_{4} V_{2}^{\prime}$, the governing equation for solving e' for both upward and downward motions is

$$
\mathrm{a}^{2}=\mathrm{b}^{2}+\mathrm{e}^{\prime 2}-2 \mathrm{~b} \mathrm{e}^{\prime} \sin (\eta+\Delta \eta)
$$

Rearranging the above equation

$$
\mathrm{e}^{\prime 2}+\mathrm{e}^{\prime}[-2 \mathrm{~b} \sin (\eta+\Delta \eta)]+\left(\mathrm{b}^{2}-\mathrm{a}^{2}\right)=0
$$

Solving the quadratic equation for $\mathrm{e}^{\prime}$,

$$
\mathrm{e}^{\prime}=\frac{2 b \sin (\eta+\Delta \eta) \pm \sqrt{4 b^{2} \sin ^{2}(\eta+\Delta \eta)-4 b^{2}+4 a^{2}}}{2}
$$

Since e' must be greater than zero,

$$
\mathrm{e}^{\prime}=b \sin (\eta+\Delta \eta)+\sqrt{a^{2}-b^{2} \cos ^{2}(\eta+\Delta \eta)}
$$

where $\mathrm{e}^{\prime}>\mathrm{e}$ when VTM moves downwards and $\mathrm{e}<\mathrm{e}$ ' when VTM moves upwards.

Also from Fig. 2.10,

$$
\begin{aligned}
& \mathrm{a}^{\prime}=\mathrm{e}^{\prime} \cos (\eta+\Delta \eta) \\
& \mathrm{b}^{\prime}=\mathrm{e}^{\prime} \sin (\eta+\Delta \eta)
\end{aligned}
$$


The local displacement of the VTM can be decomposed into both horizontal and vertical directions as

$$
\begin{aligned}
& \mathrm{r}^{\prime}=\mathrm{a}-\mathrm{a}^{\prime}=\mathrm{a}-\mathrm{e}^{\prime} \cos (\eta+\Delta \eta) \\
& \mathrm{h}^{\prime}=\mathrm{b}^{\prime}-\mathrm{b}=\mathrm{e}^{\prime} \sin (\eta+\Delta \eta)-\mathrm{b}
\end{aligned}
$$

Therefore, the horizontal $\left(\mathrm{r}_{2}\right)$ and vertical $\left(\mathrm{h}_{2}\right)$ distances between joints $\mathrm{Z}_{2}$ and $\mathrm{Z}_{3}$ can be calculated as

$$
\begin{aligned}
& \mathrm{r}_{2}=\mathrm{a}^{\prime}+24.25^{\prime \prime}=42.75^{\prime \prime}-\mathrm{r}^{\prime} \\
& \mathrm{h}_{2}=\mathrm{h}^{\prime}
\end{aligned}
$$

\subsection{Kinematic and Dynamic Analyses}

Kinematic and dynamic analyses of the Phase 3 RTD system will be carried out in this section based on the model defined in C-B notation. Moreover, the Phase 3 RTD system will be simulated using a DOE standardized software package - Tele/IGRIP.

Tele/IGRIP is an interactive, 3-D graphic simulation tool for the design and analysis of the robotic system. Any robotic system can be generated in Tele/IGRIP by creating parts, assembling the created parts, specifying the link/joint properties, and integrating with an user developed interface program to analyze the cycle time, collisions and motion constrains. The Tele/IGRIP package provides a mouse driven, point-and-click approach to simulation. There are five major components in the Tele/IGRIP software package. These include $\mathrm{CAD}$, Device, Layout, Motion and Program. CAD provides features for creating three-dimensional parts. Device is the context for creating devices using parts constructed in the CAD system. Layout is used to assemble the devices, create paths, and connect the necessary I/O. Motion contains functions to test, optimize and run 
the generated model in the workcell. Program is to generate programs using on-screen menus to script the syntax automatically into the device's program.

\subsubsection{Simulation of the Phase 3 RTD System}

By applying the Tele/IGRIP software package, 3-D solid model of each link of the Phase 3 RTD system has been created according to the dimensions specified in Table 2.2. It is also simulated to obtain the kinematic and dynamic data for the RTD system moving in the workspace. At first, with gripper 1 grasps the structural member, referring to Figs. 2.11a and $b$, and the RTD system moves from tag point $t 1(187,0,56.95)$ to $t 2$ $(20,57.5662,56.95)$ in a straight line trajectory in the same plane with elevation $Z=$ $56.95^{\prime \prime}$. In this case motion of VTM is precluded in the simulation. Similarly the second case with gripper 2 fixed and gripper 1 moves from tag point t1 to tag point $t 2$ is illustrated in Figs. 2.12a and b.

Additionally, to observe the effect of the vertical motion caused by the VTM, one arbitrary trajectory in the workspace defined by five tag points $t 1$ through $t 5$, Table 2.4 , is selected. By following this trajectory, the RTD system grasps the same structural member at the end of its motion. Two different cases have been simulated for studying its kinematic and dynamic behaviors. First, the gripper 1 grasps one of the structural members and gripper 2 follows the predetermined trajectory and finally grasps the same structural member, Figs. 2.13a to e. Second, Figs. 2.14a to e, gripper 2 originally grasps one structural member and gripper 1 approaches the same structural member by following the given path. 
Table 2.4 Tag points through which the Phase 3 RTD system follows in workspace

\begin{tabular}{|c|c|c|c|}
\hline tag points & $\mathrm{X}$ (inches) & $\mathrm{Y}$ (inches) & $\mathrm{Z}$ (inches) \\
\hline $\mathrm{t} 1$ & 187.00 & 0 & 56.95 \\
\hline $\mathrm{t} 2$ & 180.080 & 34.6565 & 59.125 \\
\hline $\mathrm{t} 3$ & 140.002 & 80.8650 & 61.5 \\
\hline $\mathrm{t} 4$ & 80 & 92.4173 & 63.875 \\
\hline $\mathrm{t} 5$ & 0 & 0 & 66.25 \\
\hline
\end{tabular}




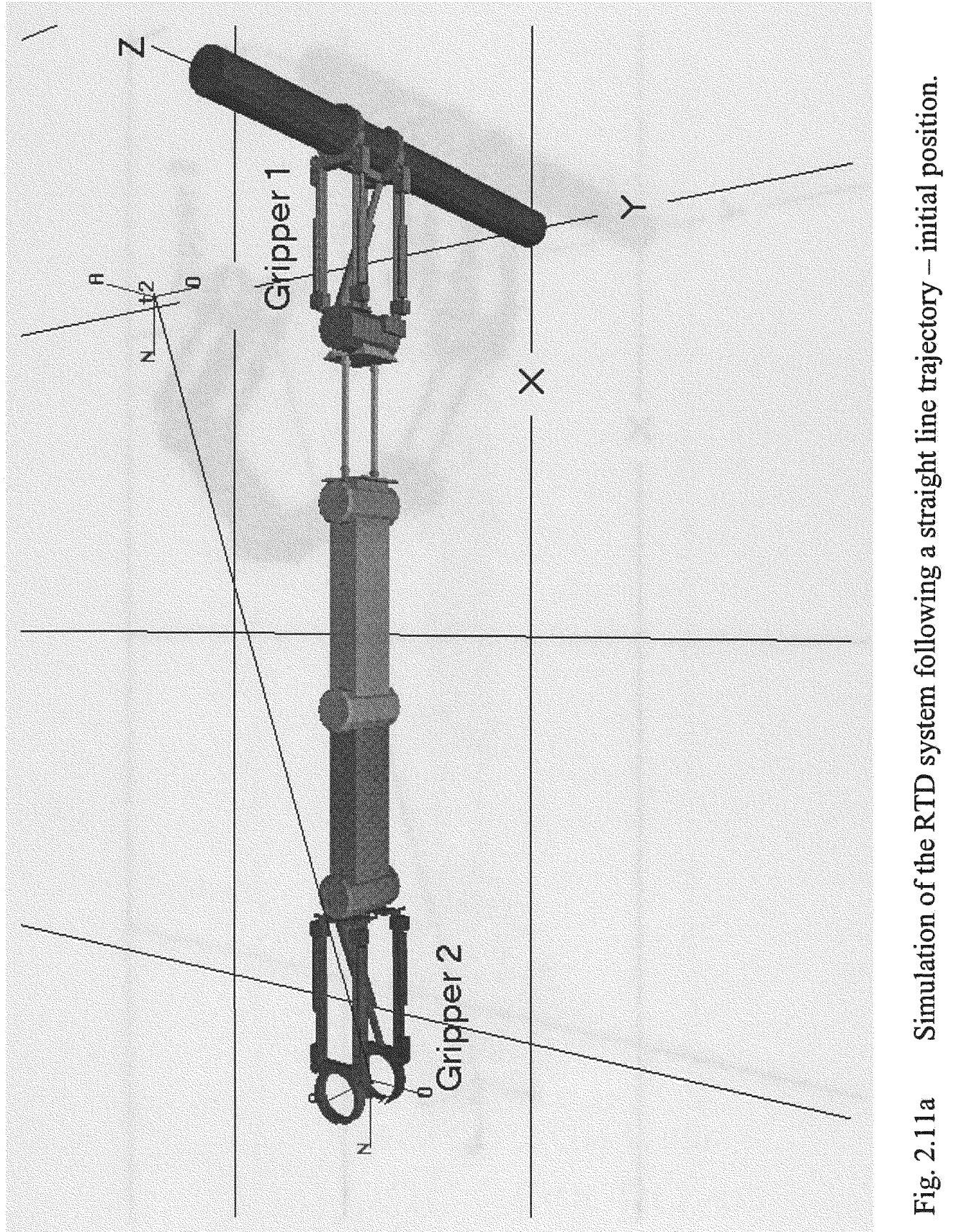




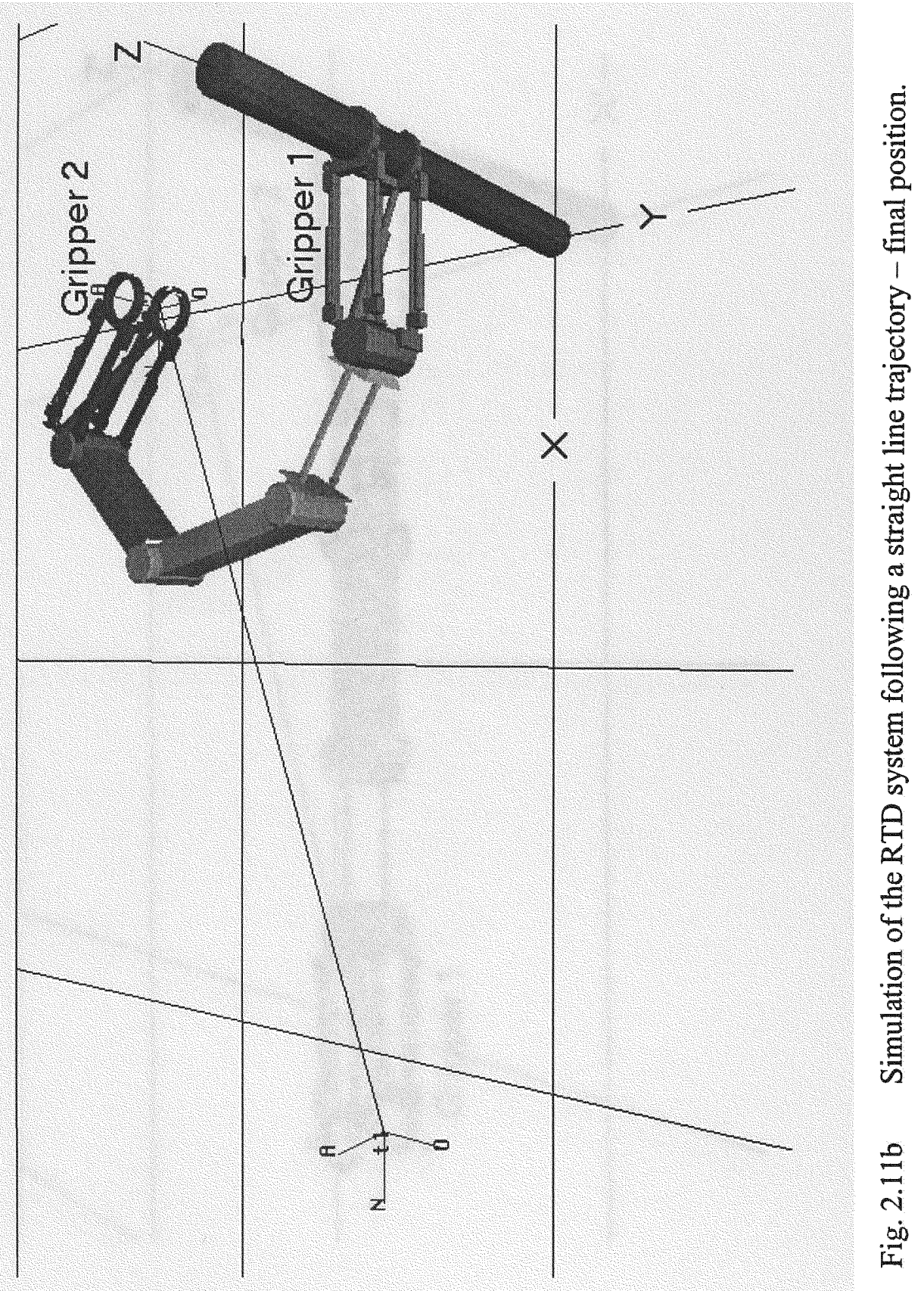




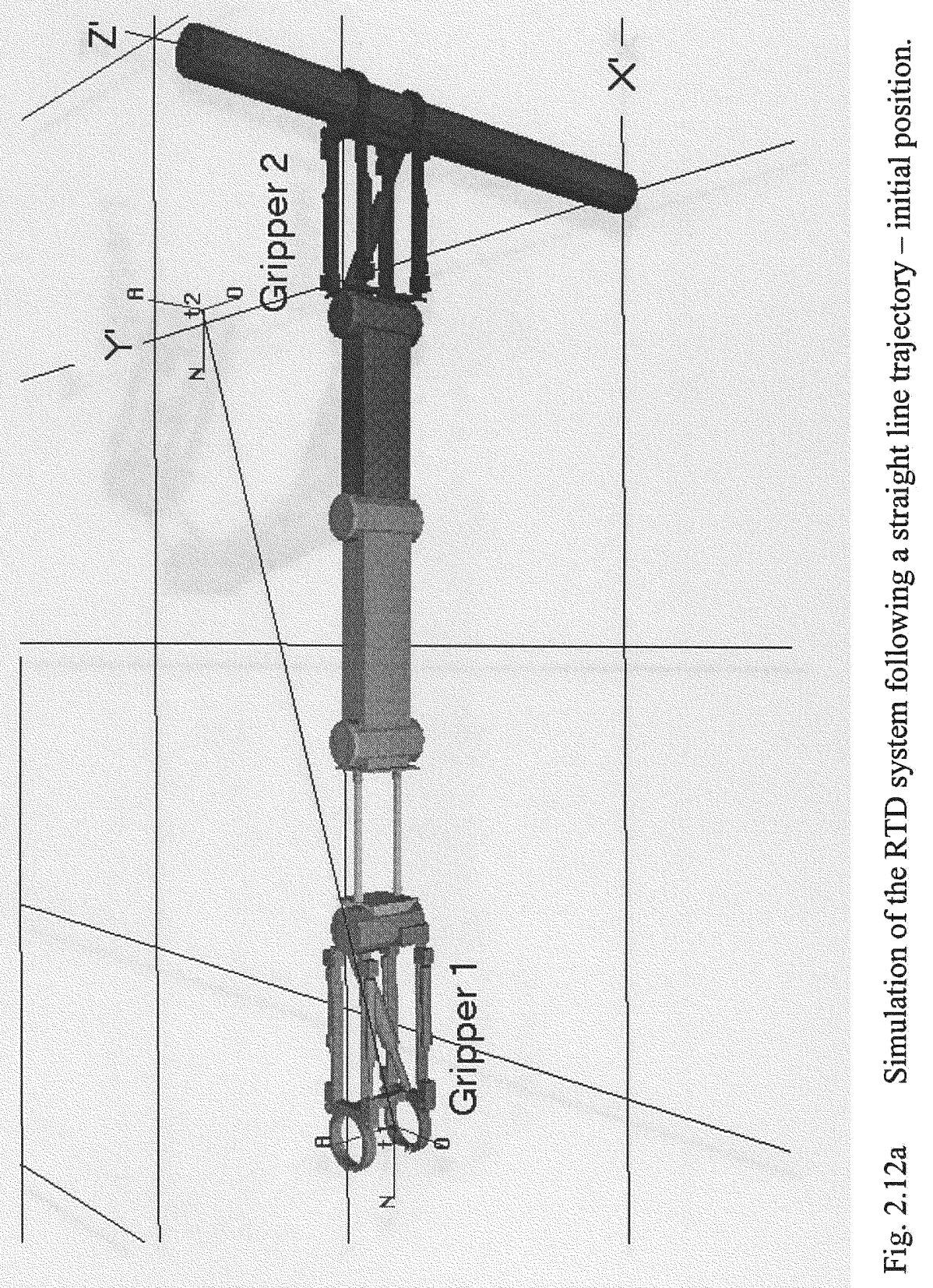




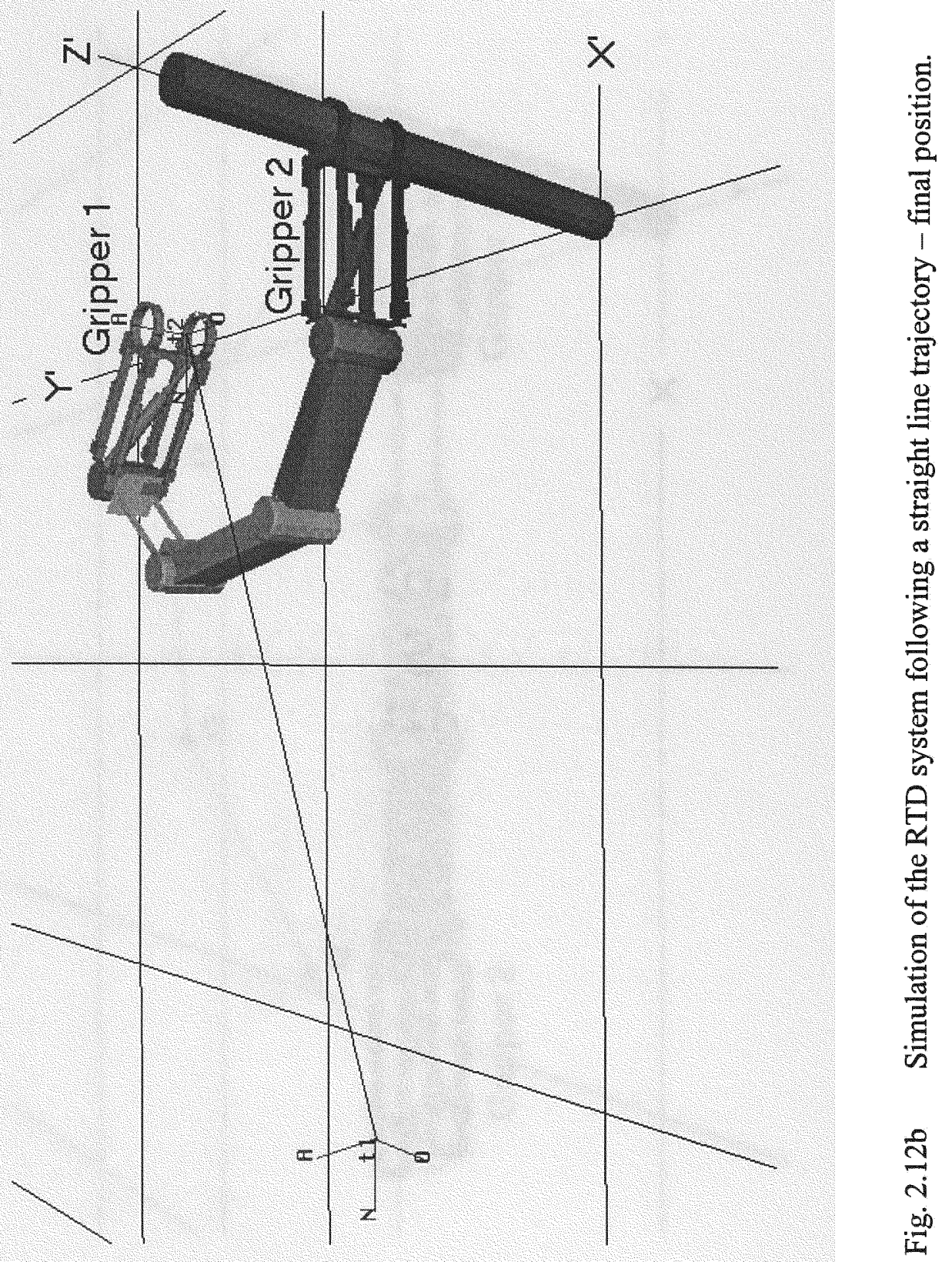




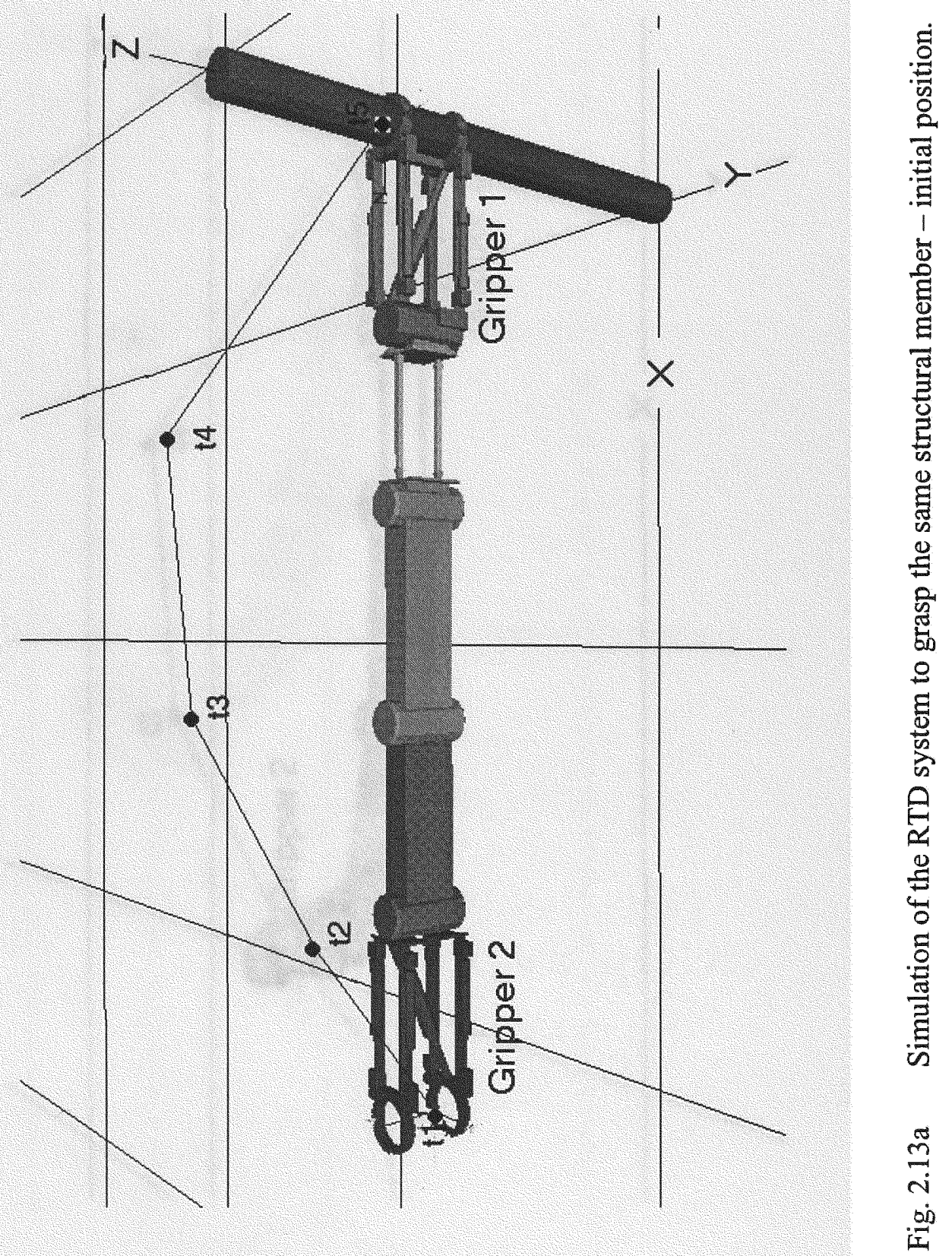




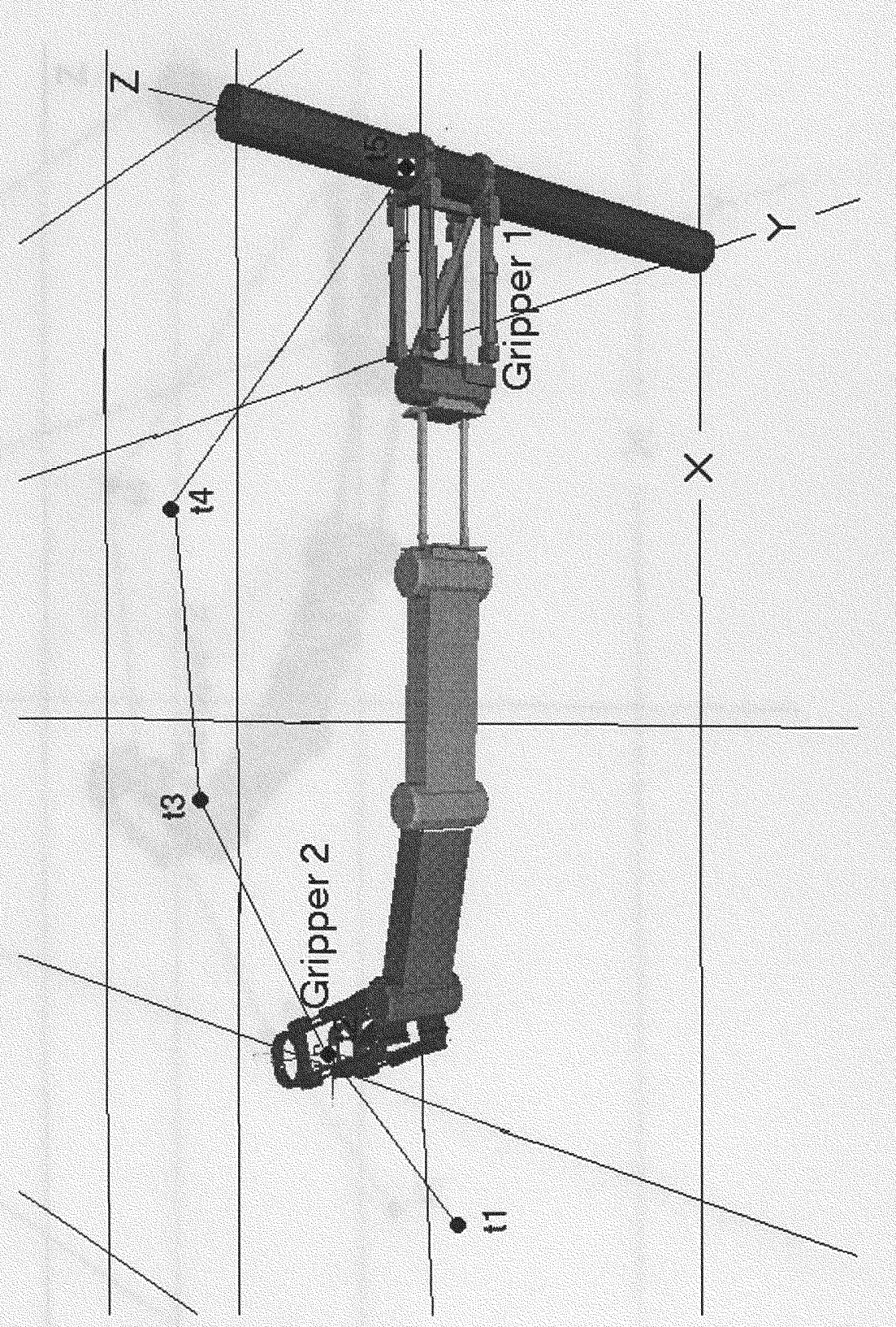

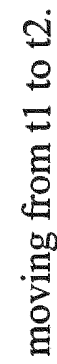

!

葛

苨

홀

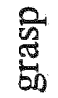

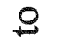

曾

$\theta$

$\stackrel{\Xi}{\leftrightarrows}$

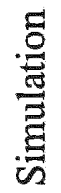

ले 


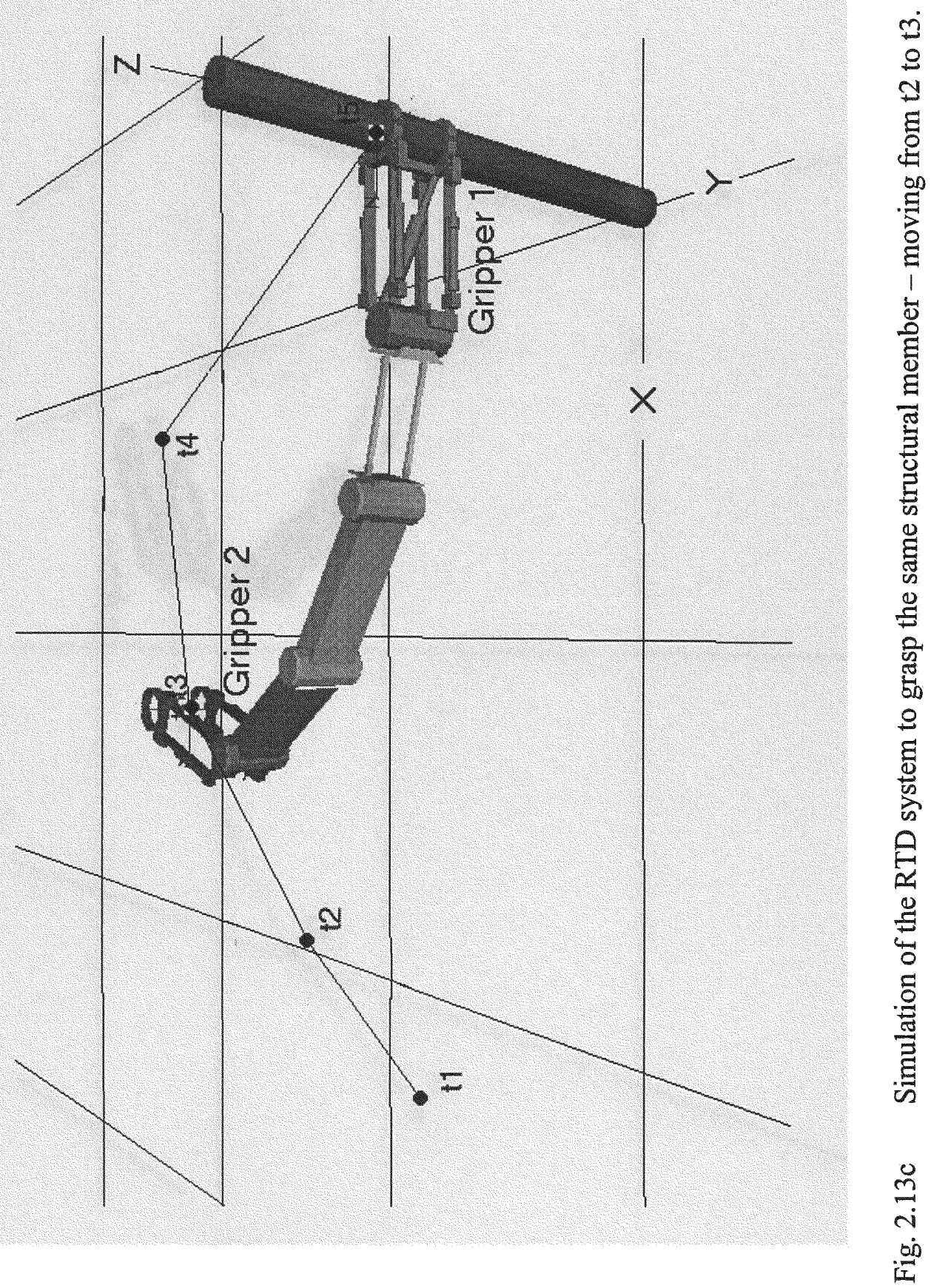




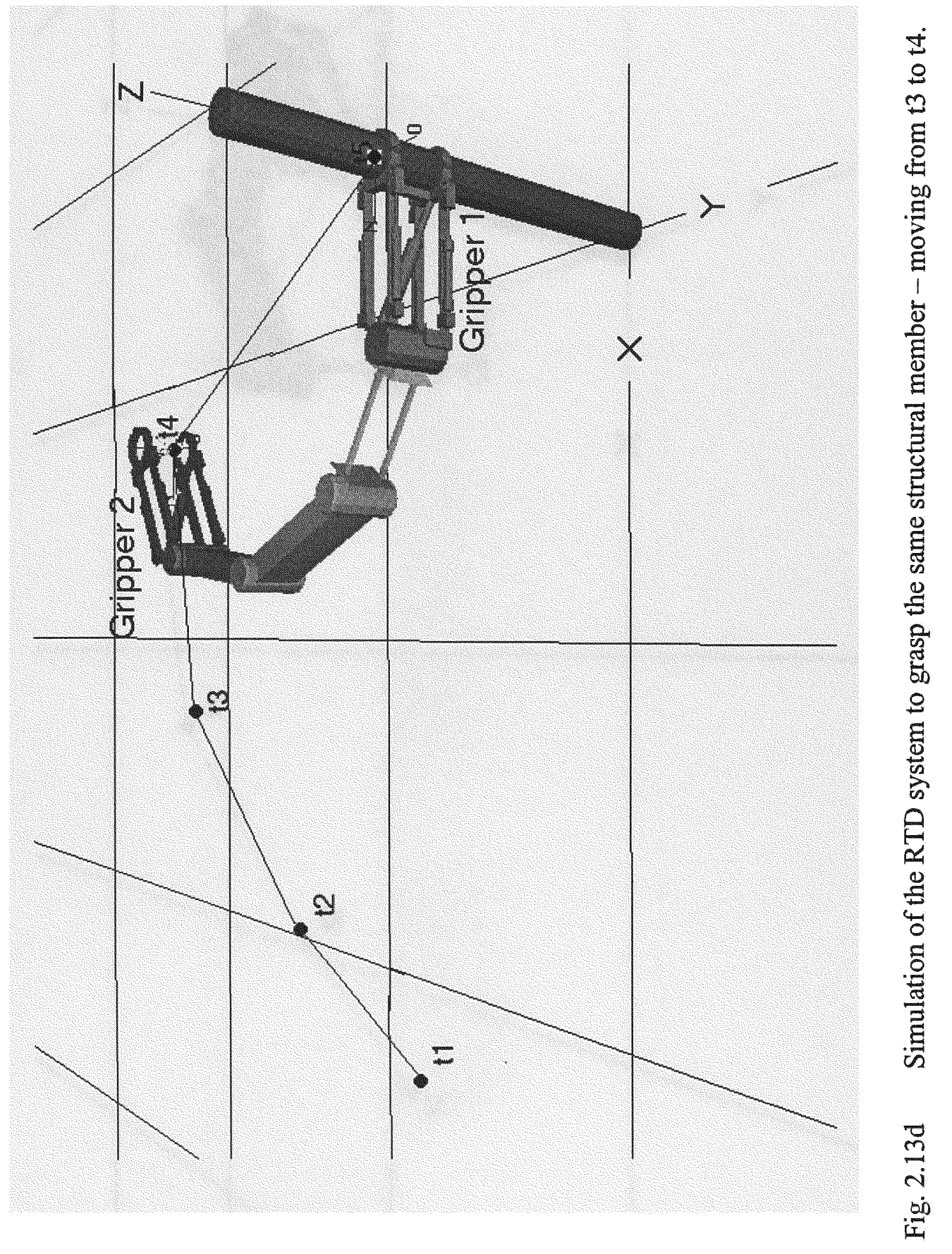




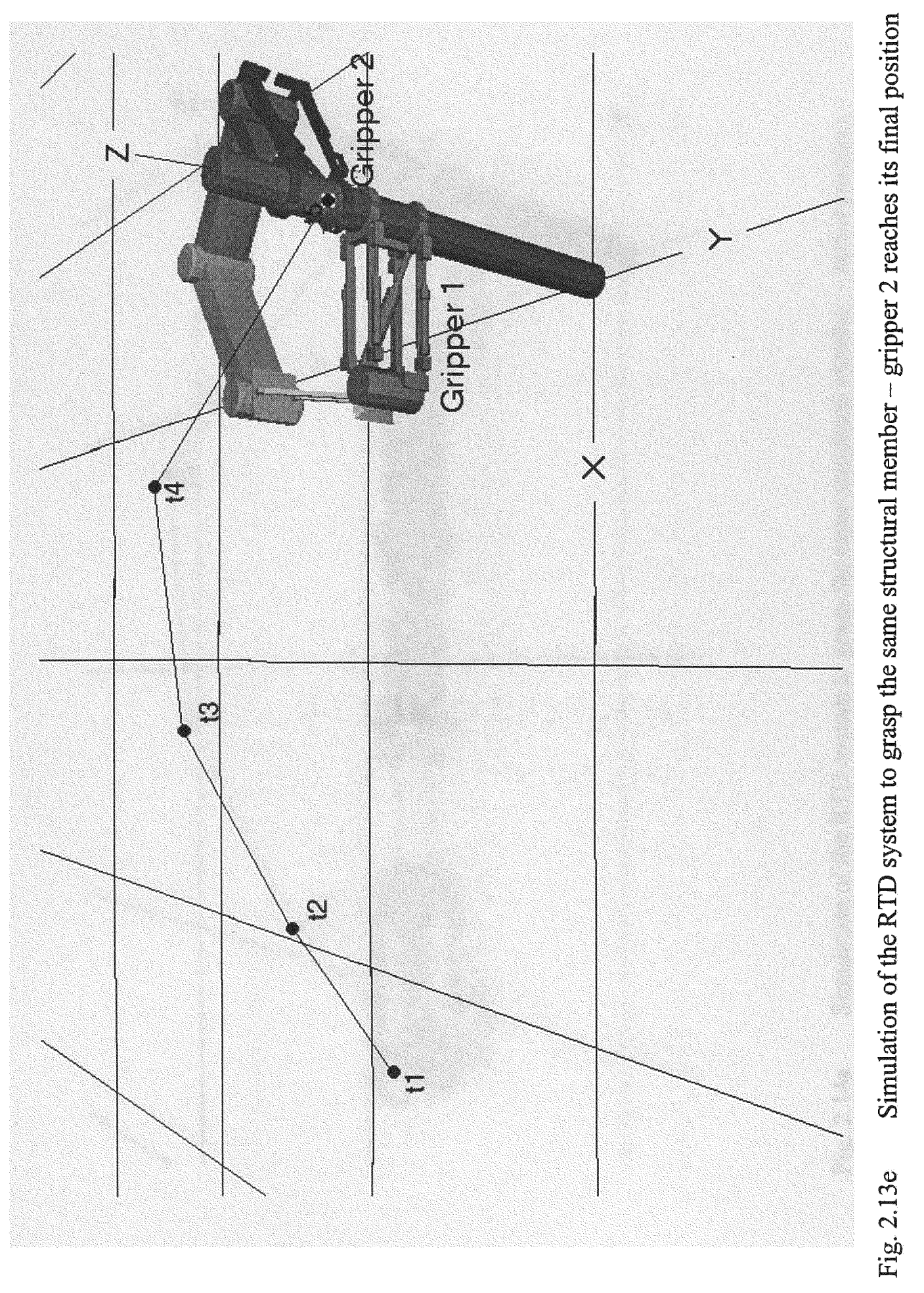




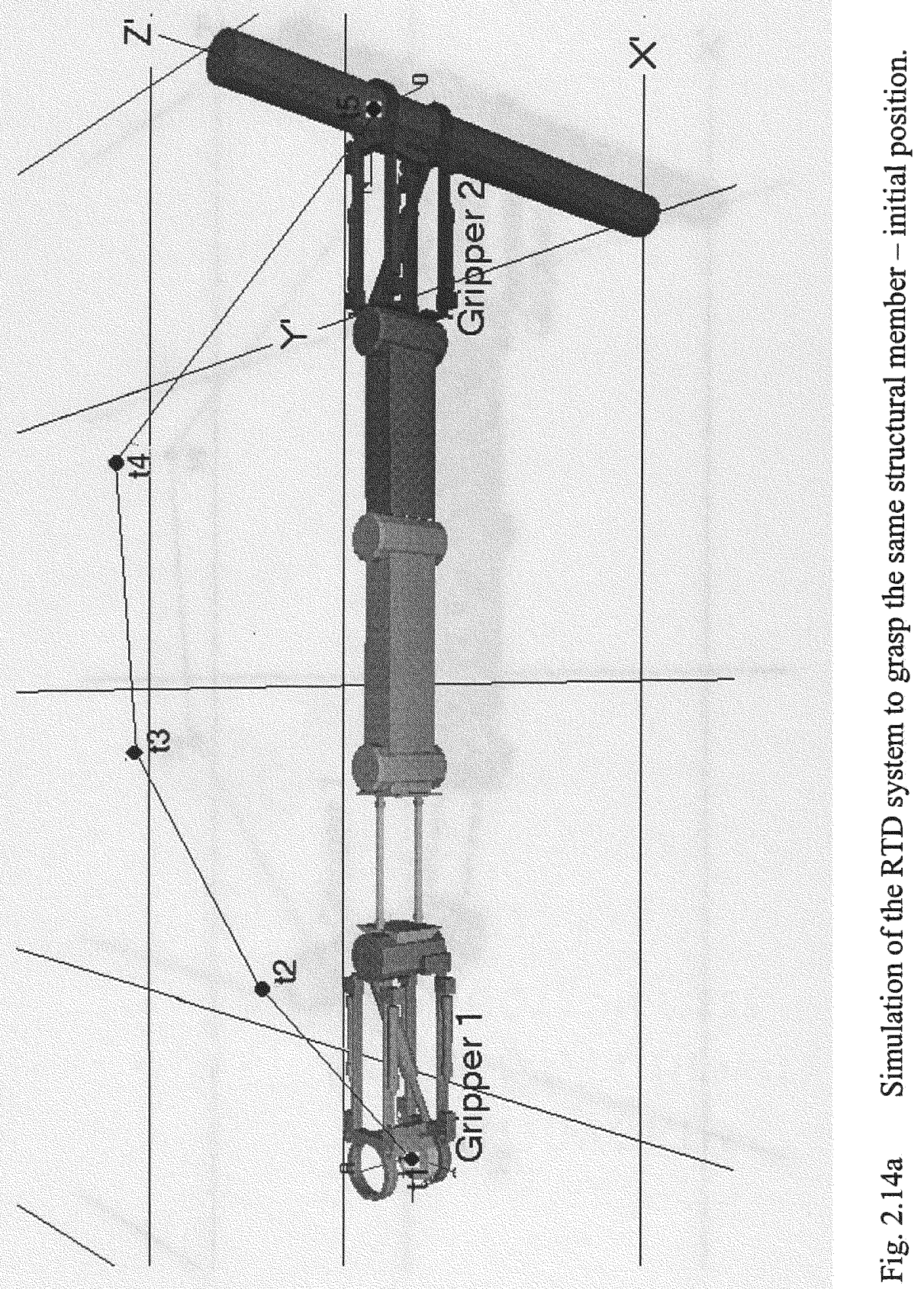




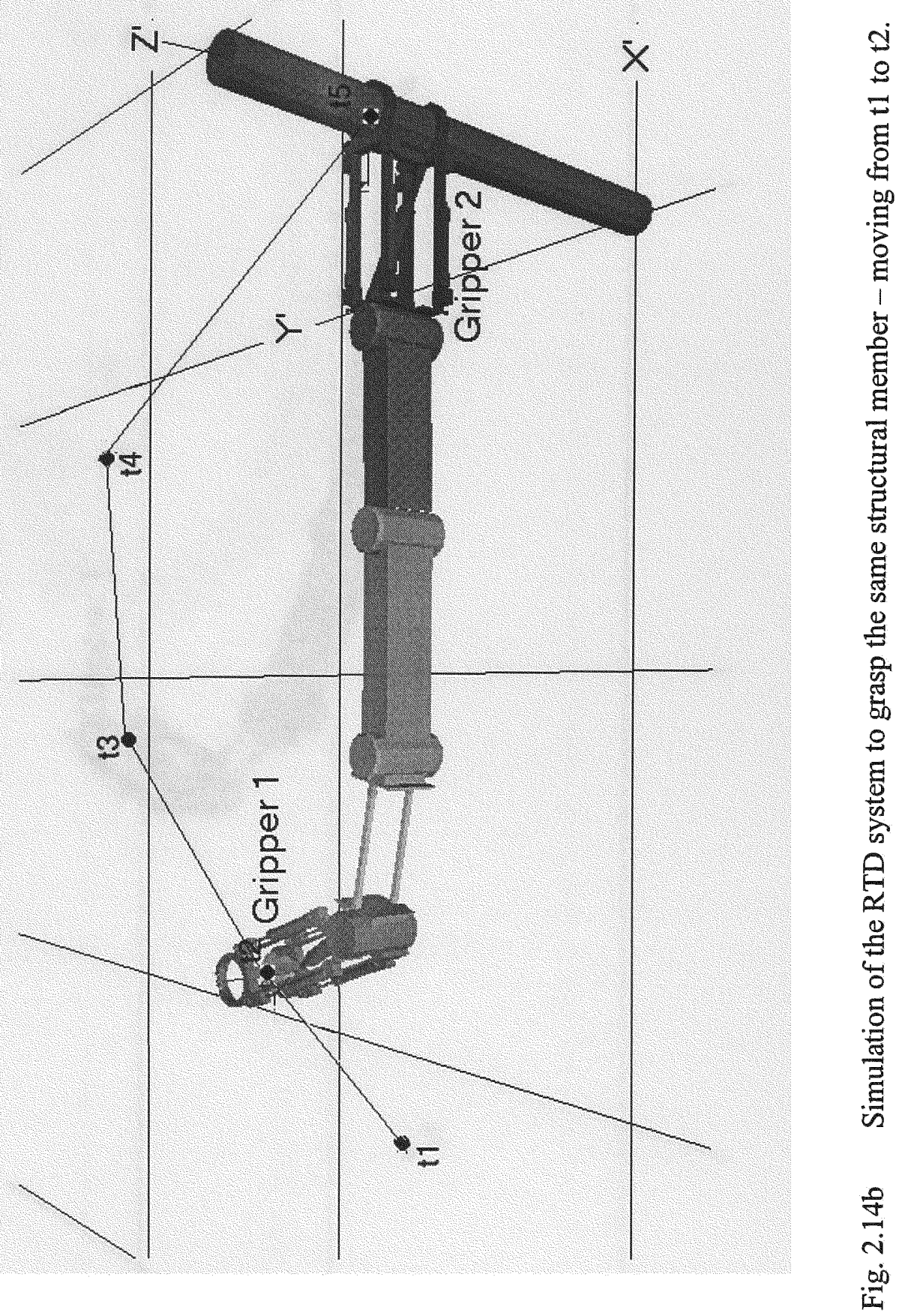






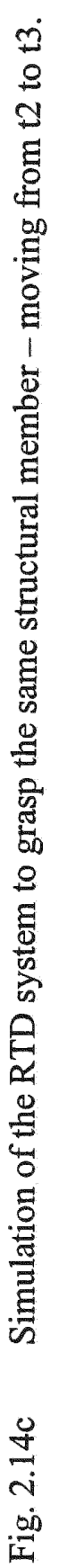




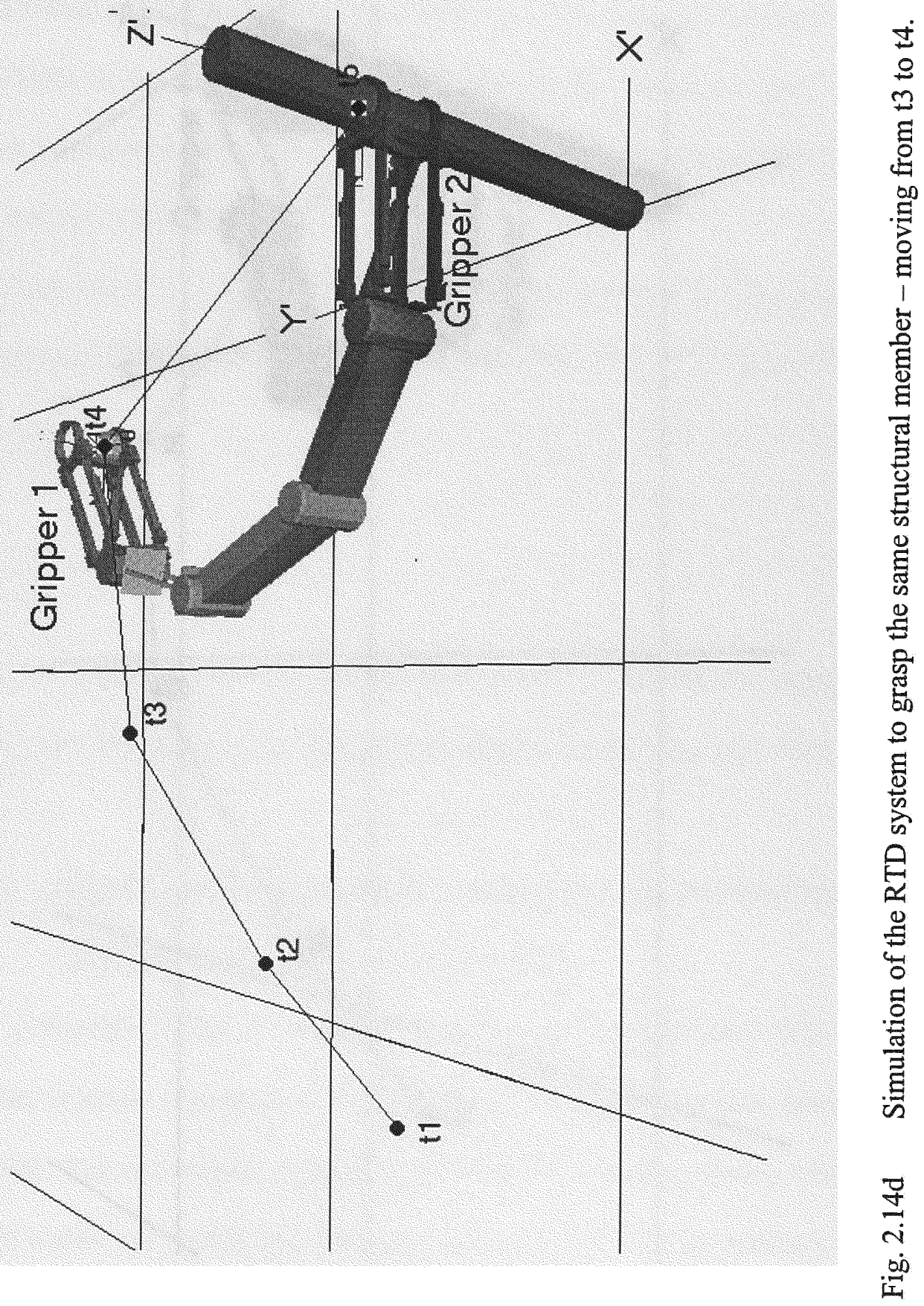




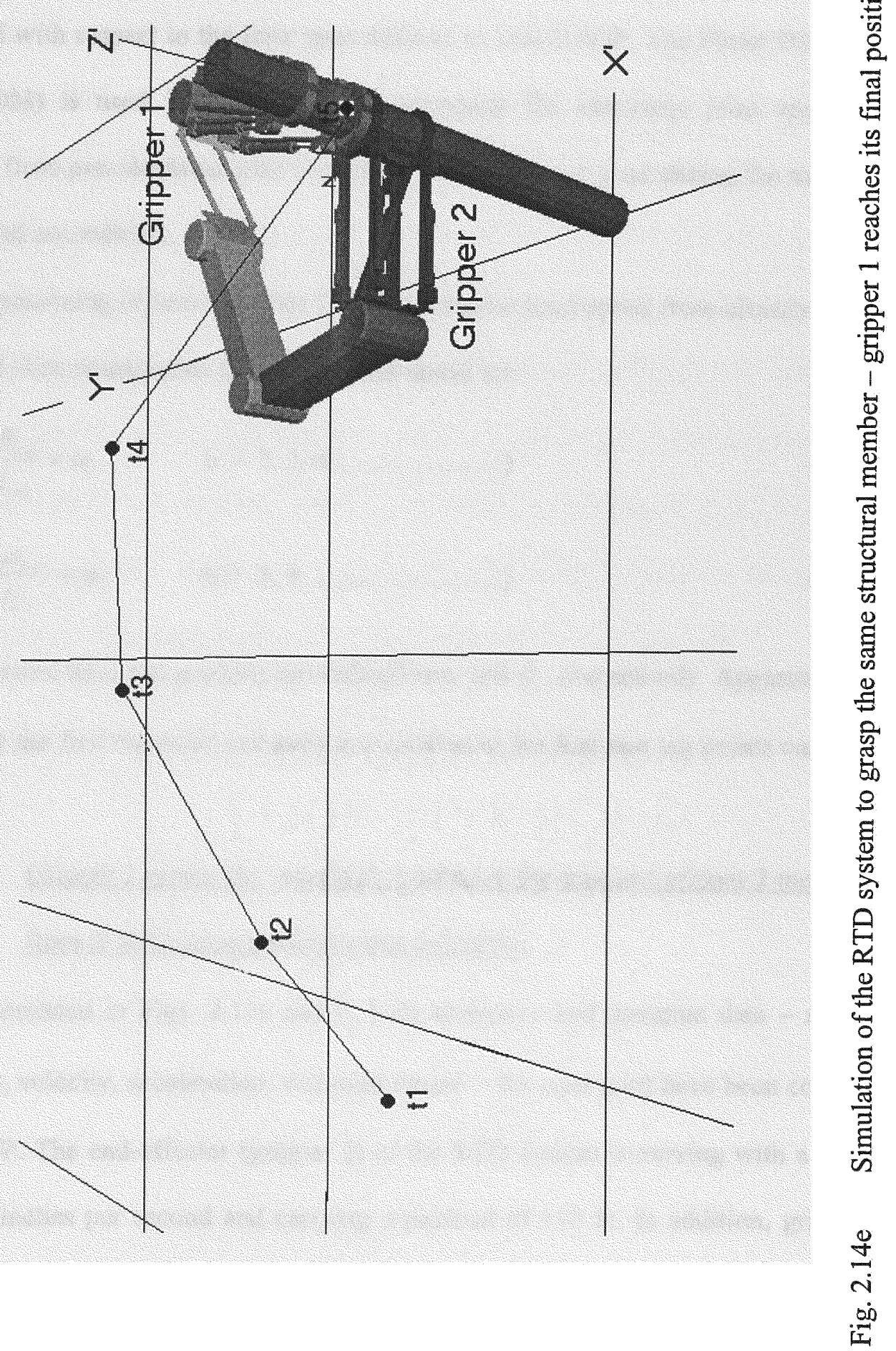




\subsubsection{Numerical Examples}

Throughout the above mentioned simulations, both kinematic and dynamic data are collected with respect to the time span defined in Tele/IGRIP. The Finite Difference Method (FDM) is used in Tele/IGRIP to determine the kinematic joint speed and acceleration from precalculated joint positions. Torque of each joint during the motion is also calculated accordingly.

The governing equations of the FDM to compute joint speed from already known position, and joint acceleration from calculated speed are

$$
\begin{array}{ll}
\frac{\theta_{i}-\theta_{i-1}}{t_{i}-t_{i-1}}=\omega_{i} & (i=2,3,4 \ldots \ldots \ldots \ldots \ldots . . . \ldots) \\
\frac{\omega_{i}-\omega_{i-1}}{t_{i}-t_{i-1}}=\alpha_{i} & (i=3,4 \ldots \ldots \ldots \ldots \ldots \ldots)
\end{array}
$$

The initial time and position are defined as $\mathrm{t}_{1}$ and $\theta_{1}$, respectively. Apparently, the joint speed at the first tag point and joint acceleration at the first two tag points cannot be calculated.

Example 1: Gripper 1 grasps the structural member in the tank and gripper 2 moves from $\mathrm{t} 1$ to $\mathrm{t} 2$ along a straight line trajectory.

As simulated in Figs. $2.11 \mathrm{a}$ and $\mathrm{b}$, both kinematic and dynamic data - angular displacement, velocity, acceleration, and joint torque - for each joint have been collected in Tele/IGRIP. The end-effector (gripper 2) of the RTD system is moving with a steady speed of 20 inches per second and carrying a payload of $110 \mathrm{lb}$. In addition, gripper 2 moves from its initial position $\mathrm{t} 1$ to final position $\mathrm{t} 2$ within 18 seconds, which is defined automatically by Tele/IGRIP. The graph in Fig. $2.15 \mathrm{a}$ represents the angular 
displacement of gripper 2 relative to joint $2^{\prime}$. By analyzing this graph, angular displacement first increases in magnitude till it reaches its maximum limit $\left(135^{\circ}\right)$ half the way of the motion and then starts decreasing. The curve shown in Fig. 2.15b gives the angular velocity of the end-effector. The angular velocity is decreasing to zero degree per second at middle of the path and, afterwards, changing direction until it reaches the maximum angular velocity of $10 \mathrm{deg} / \mathrm{sec}$. To interpret Fig. $2.15 \mathrm{c}$, it shows that gripper 2 decelerates rapidly within the first 1.25 seconds and then gradually decelerates to stall in 18 seconds. Torque of joint 2', connected to gripper 2, is shown in Fig. 2.15d. It decreases steeply in the first 2 seconds and reaches a relatively minimum possible joint torque. Similar interpretations can be made joints 3 ', 3, and 2 by referring to Figs. 2.16a to d, Figs. 2.17a to d, and Figs. 2.18a to d, respectively. The torque applied to the structural member, exerted by gripper 1, is identical to the torque of joint 2, Fig. $2.18 \mathrm{~d}$. 


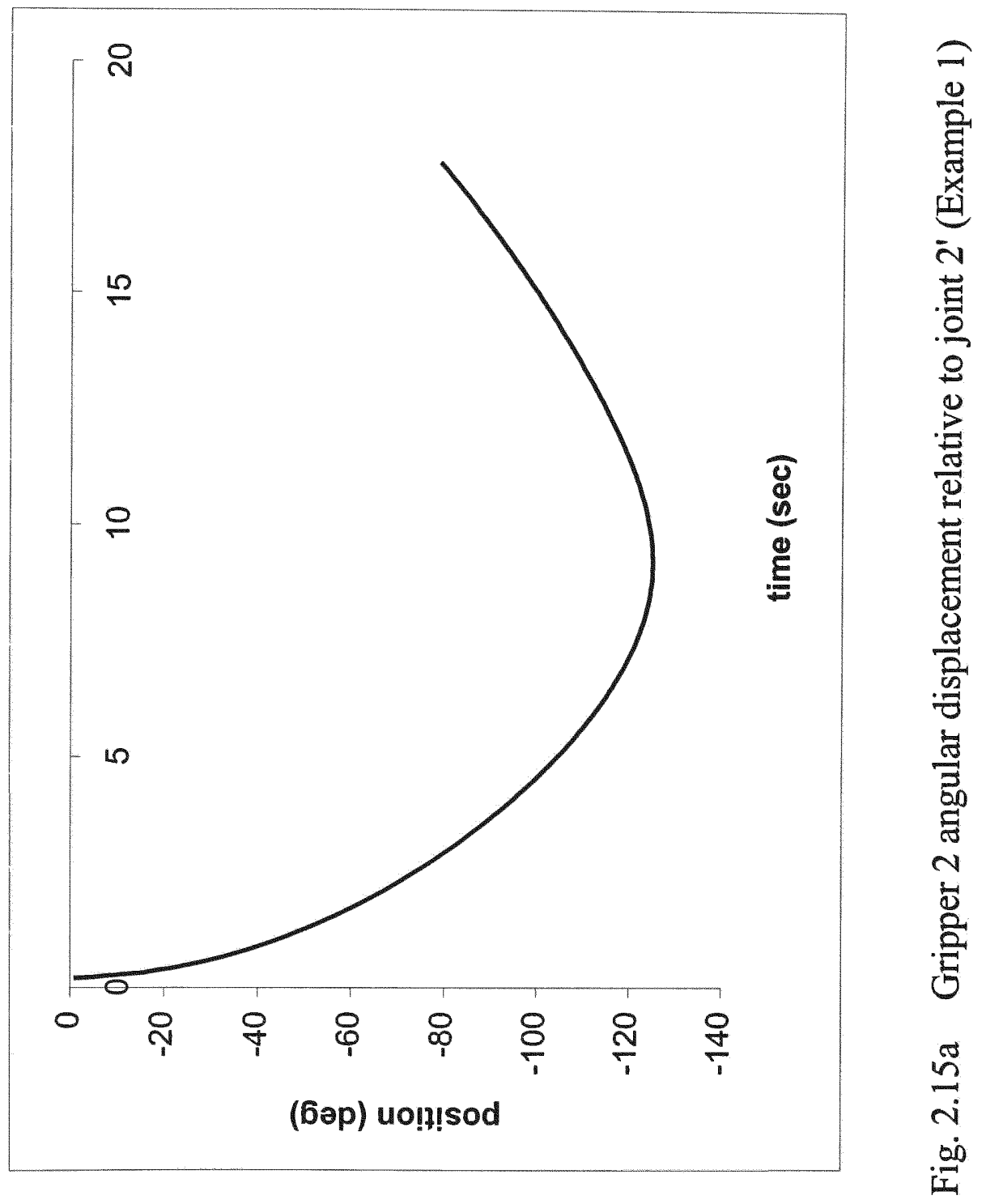




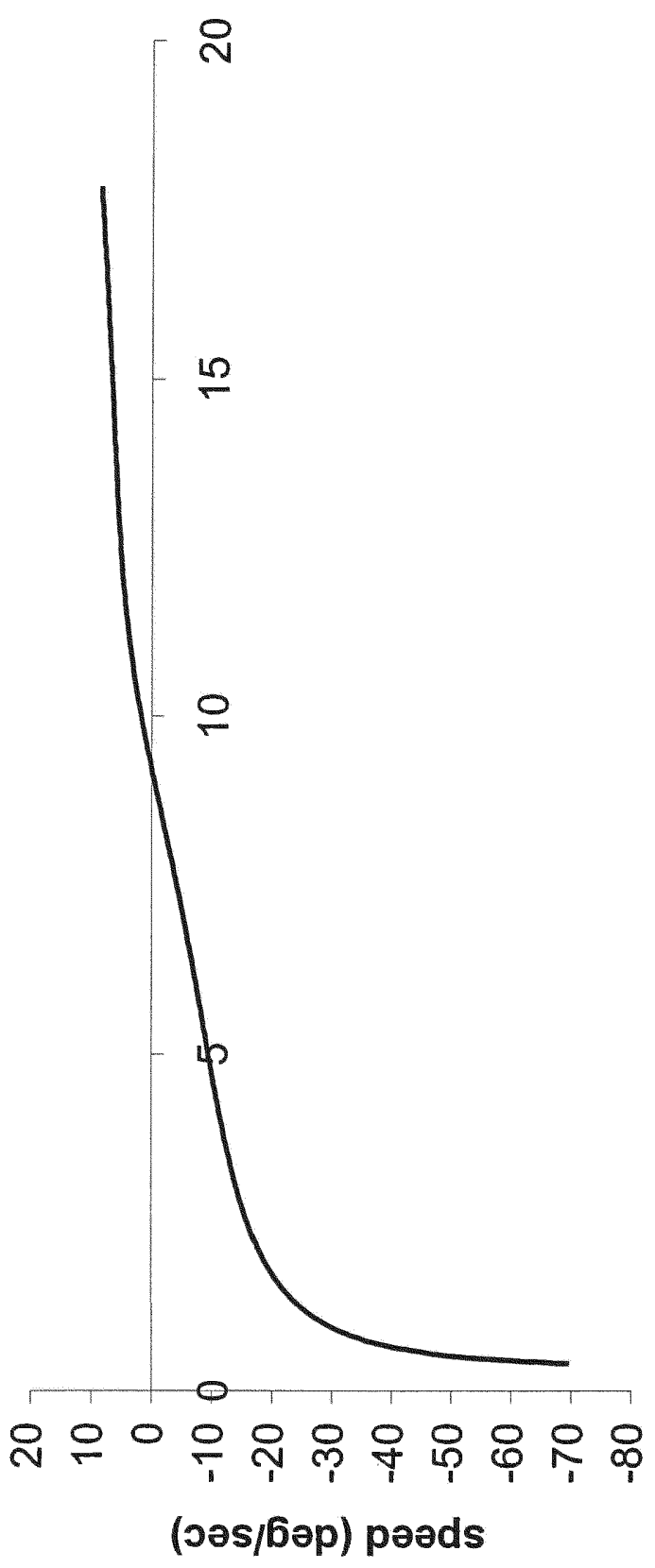

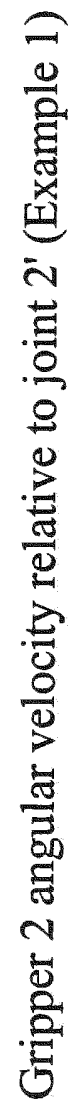

है 


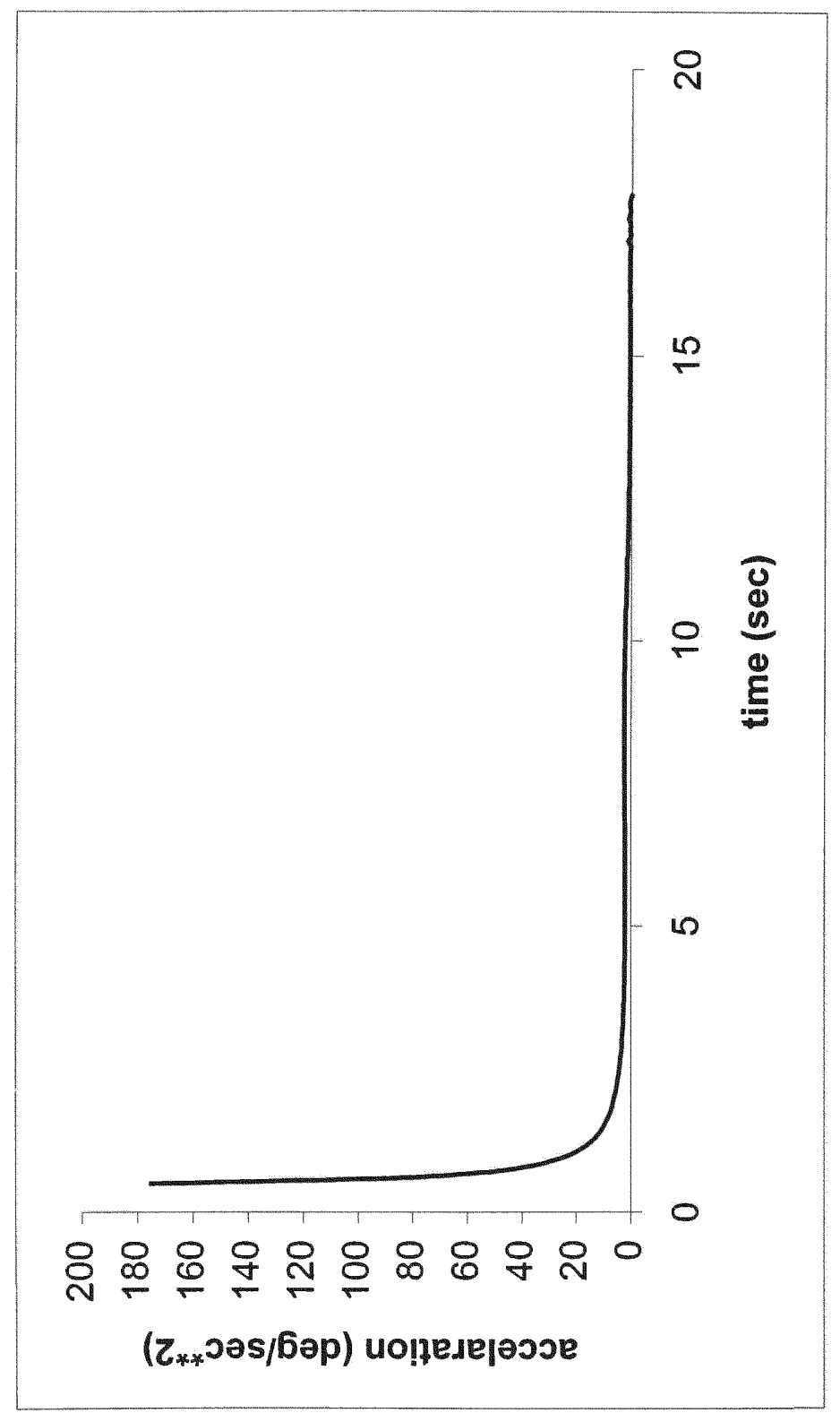

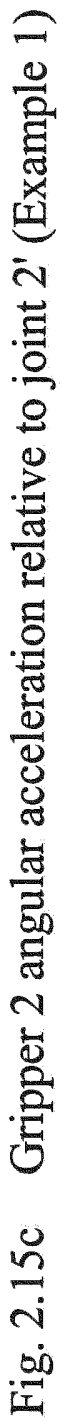




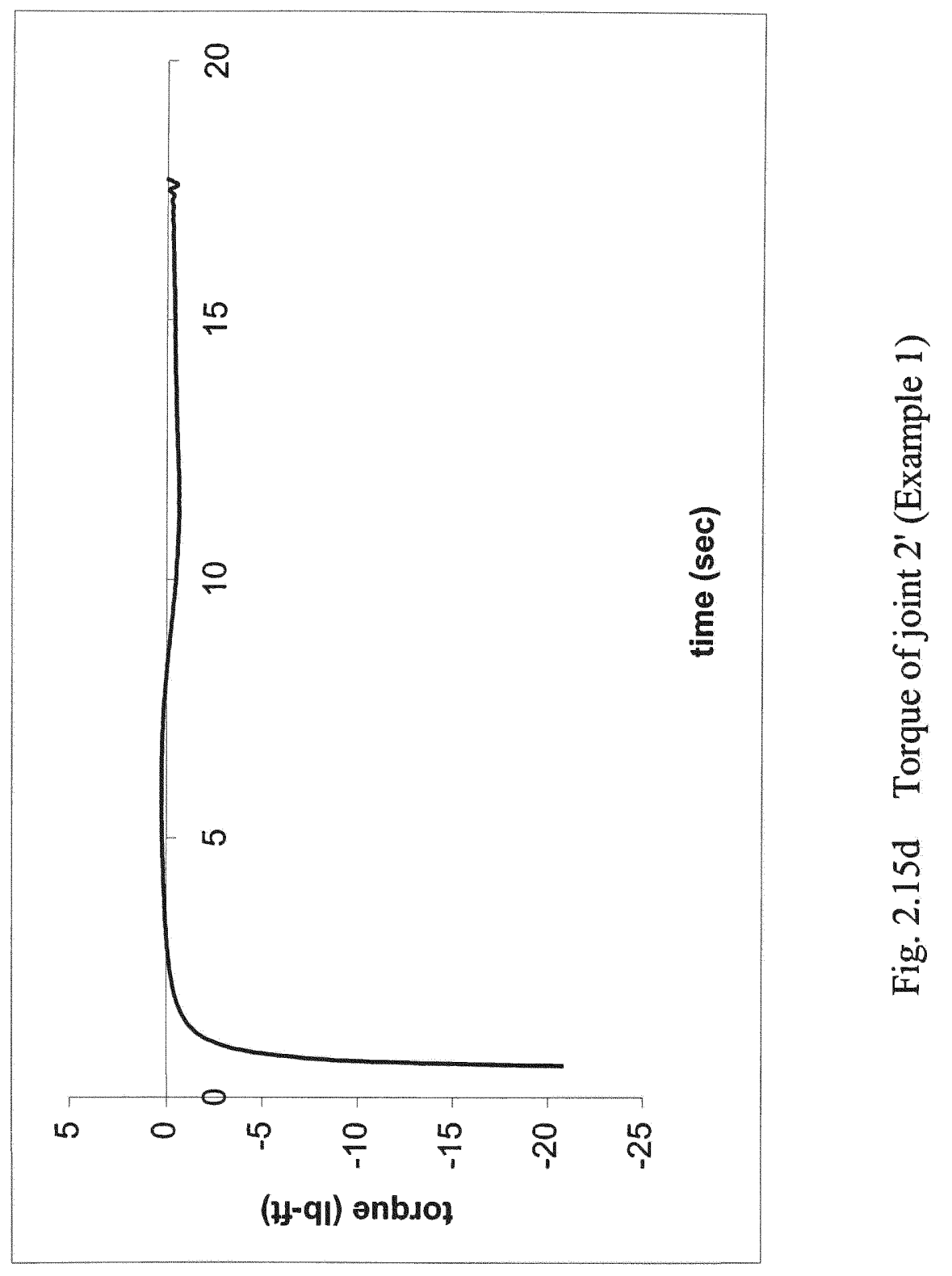




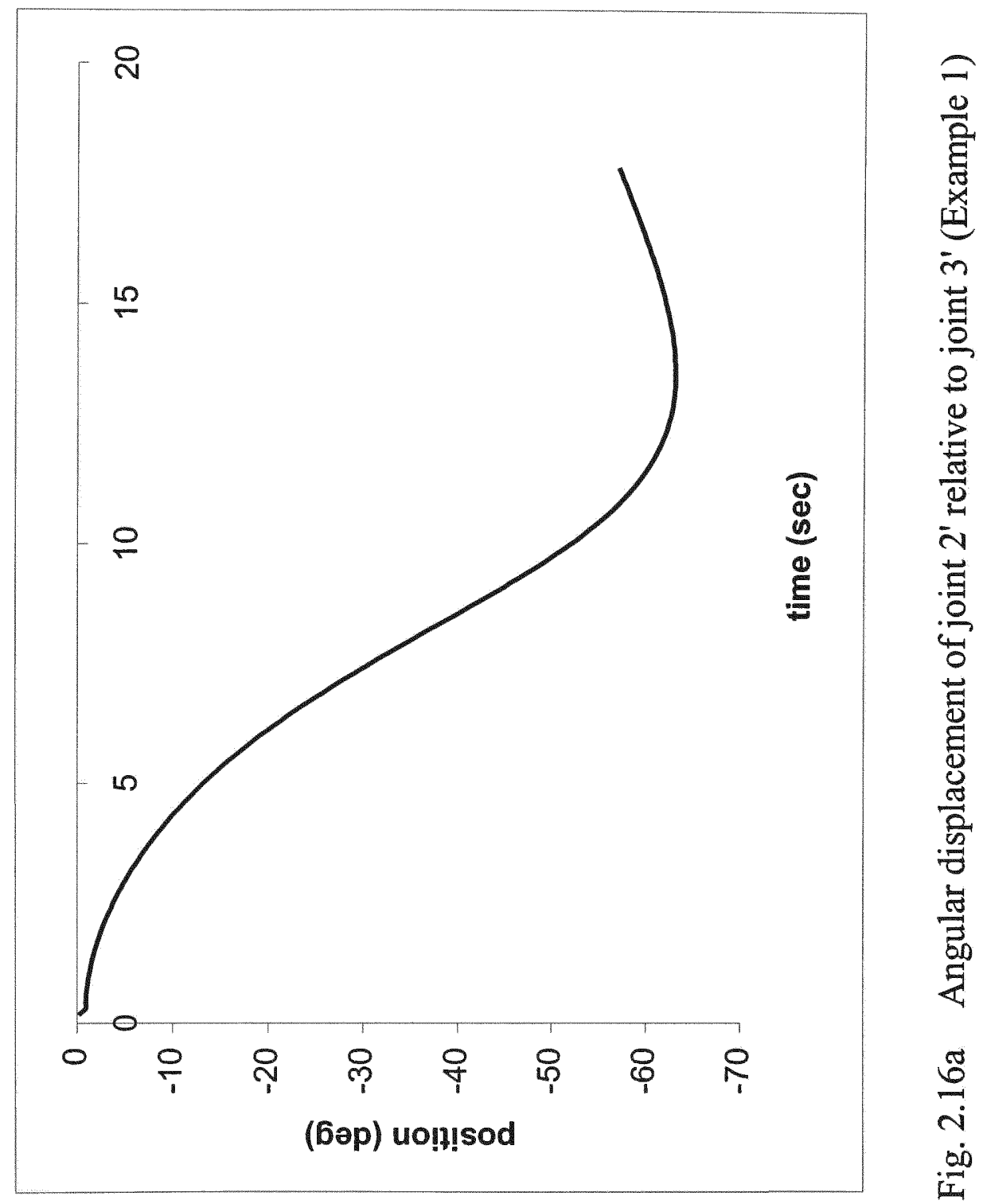




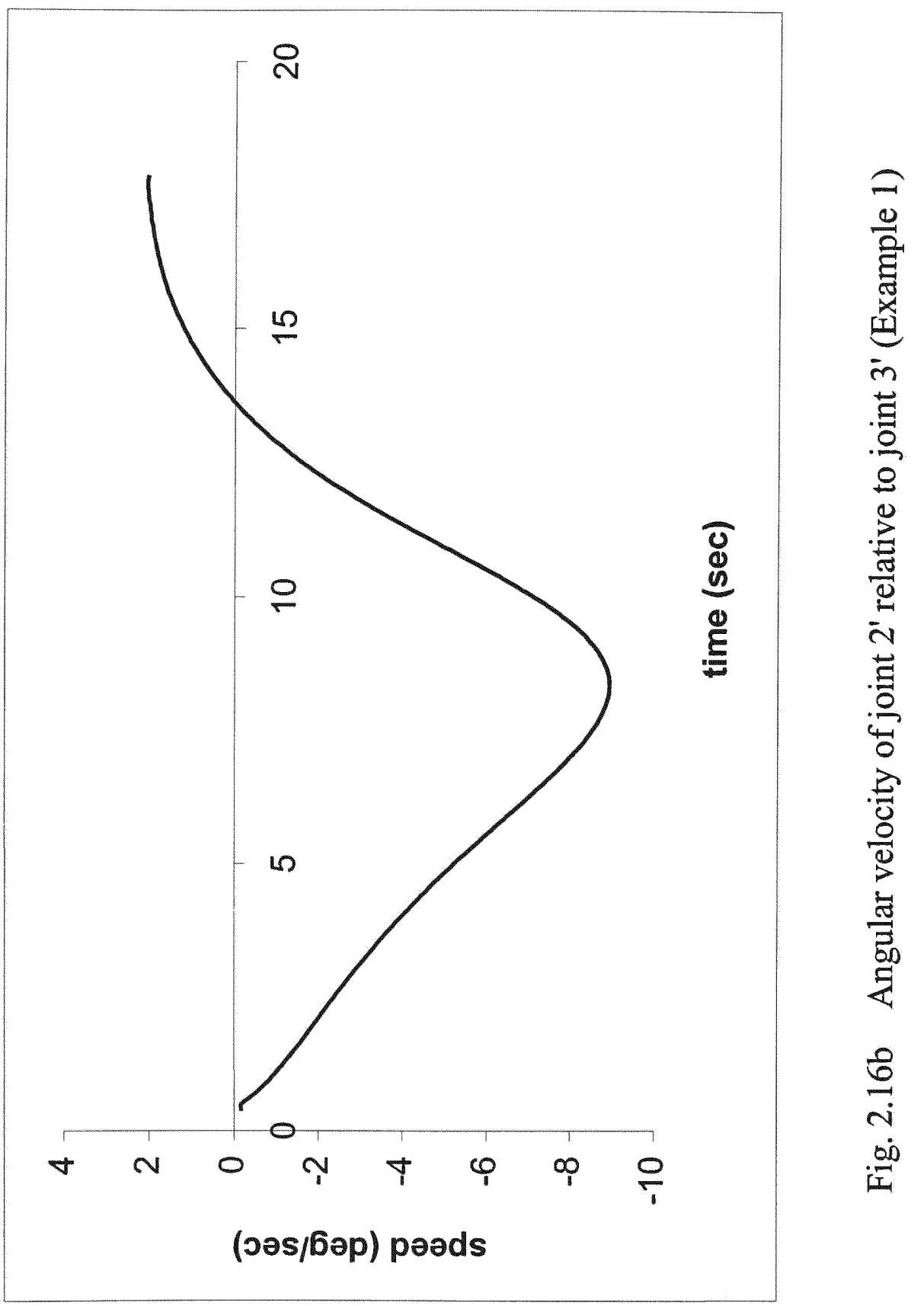




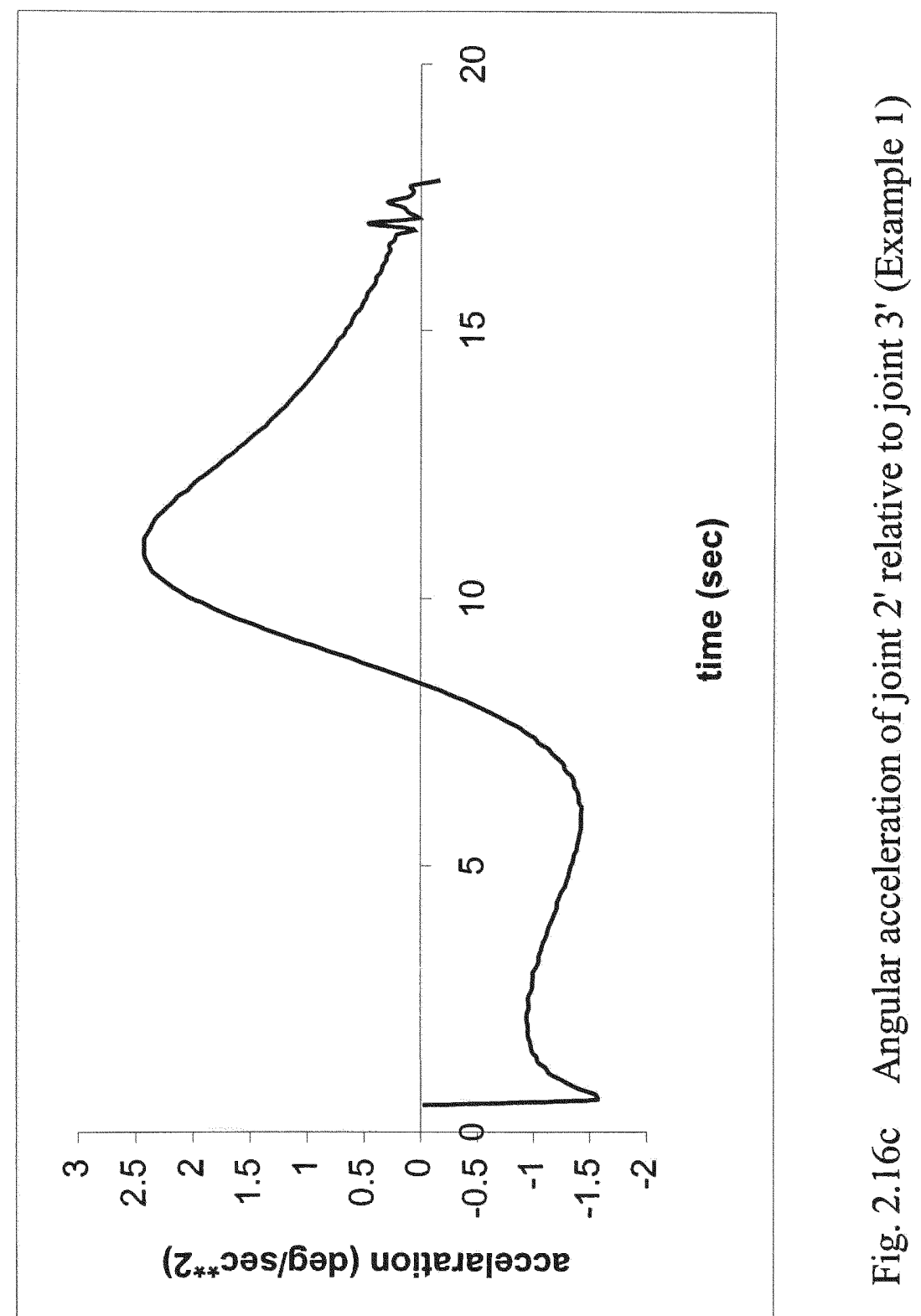




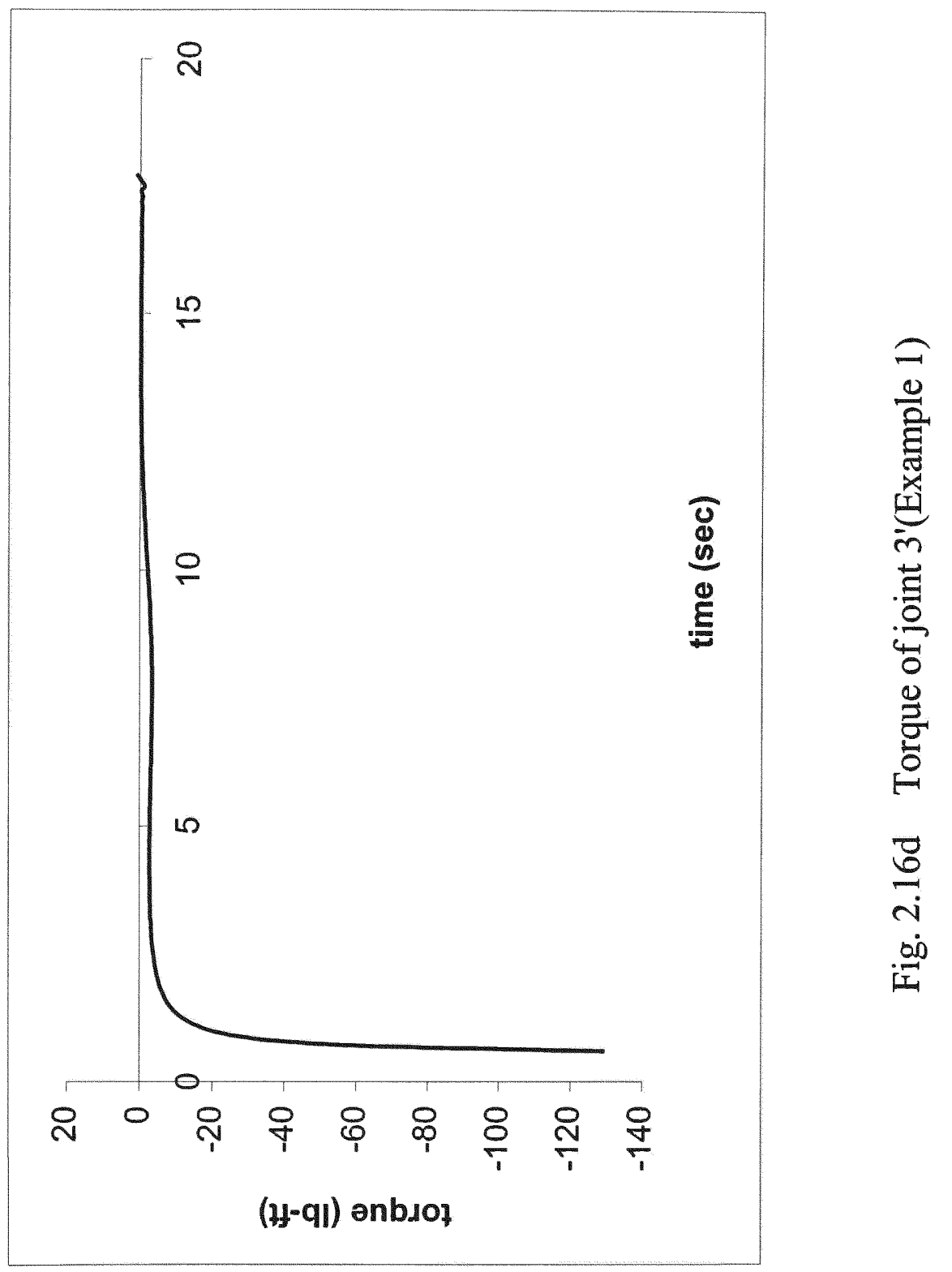




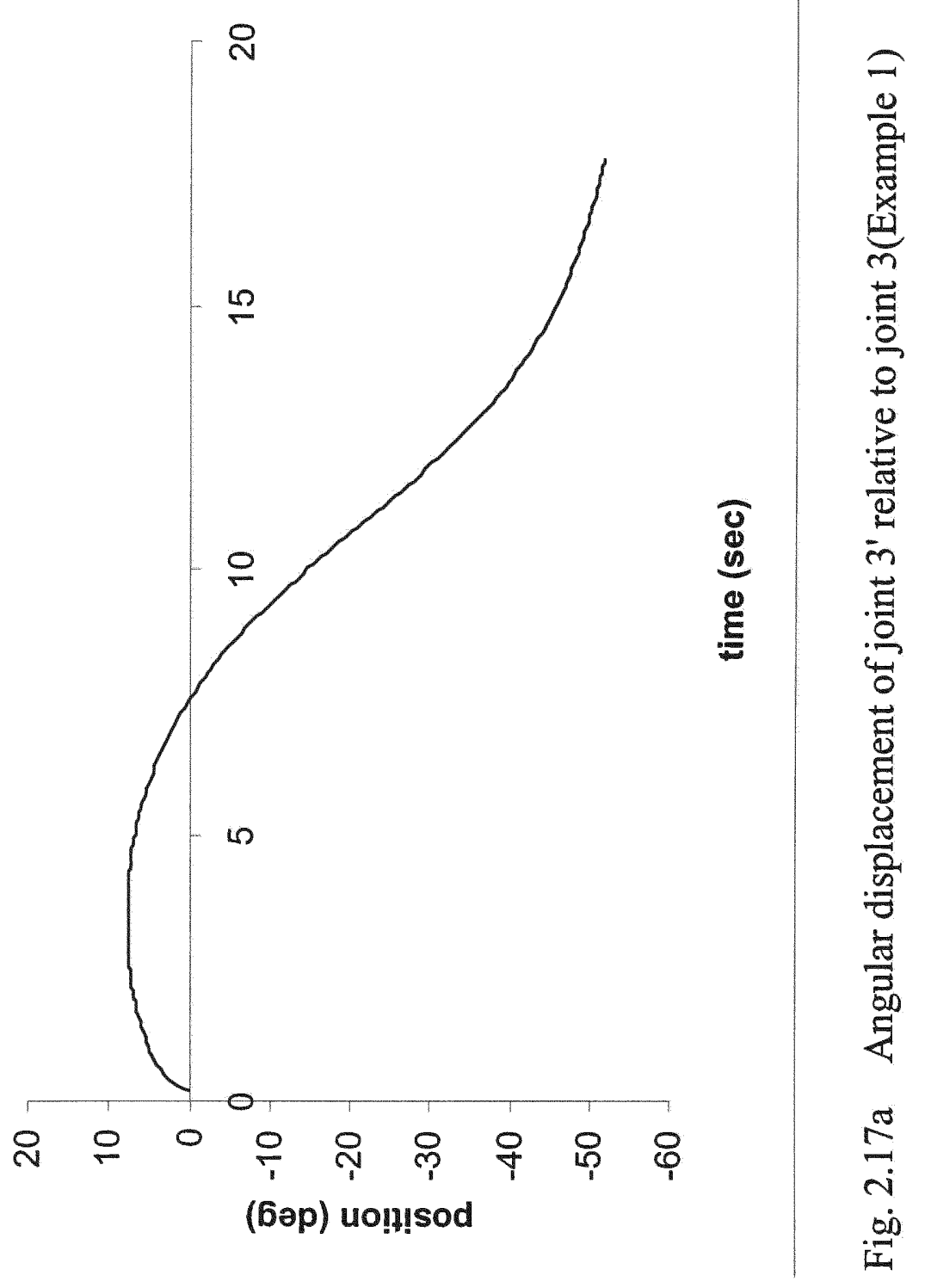




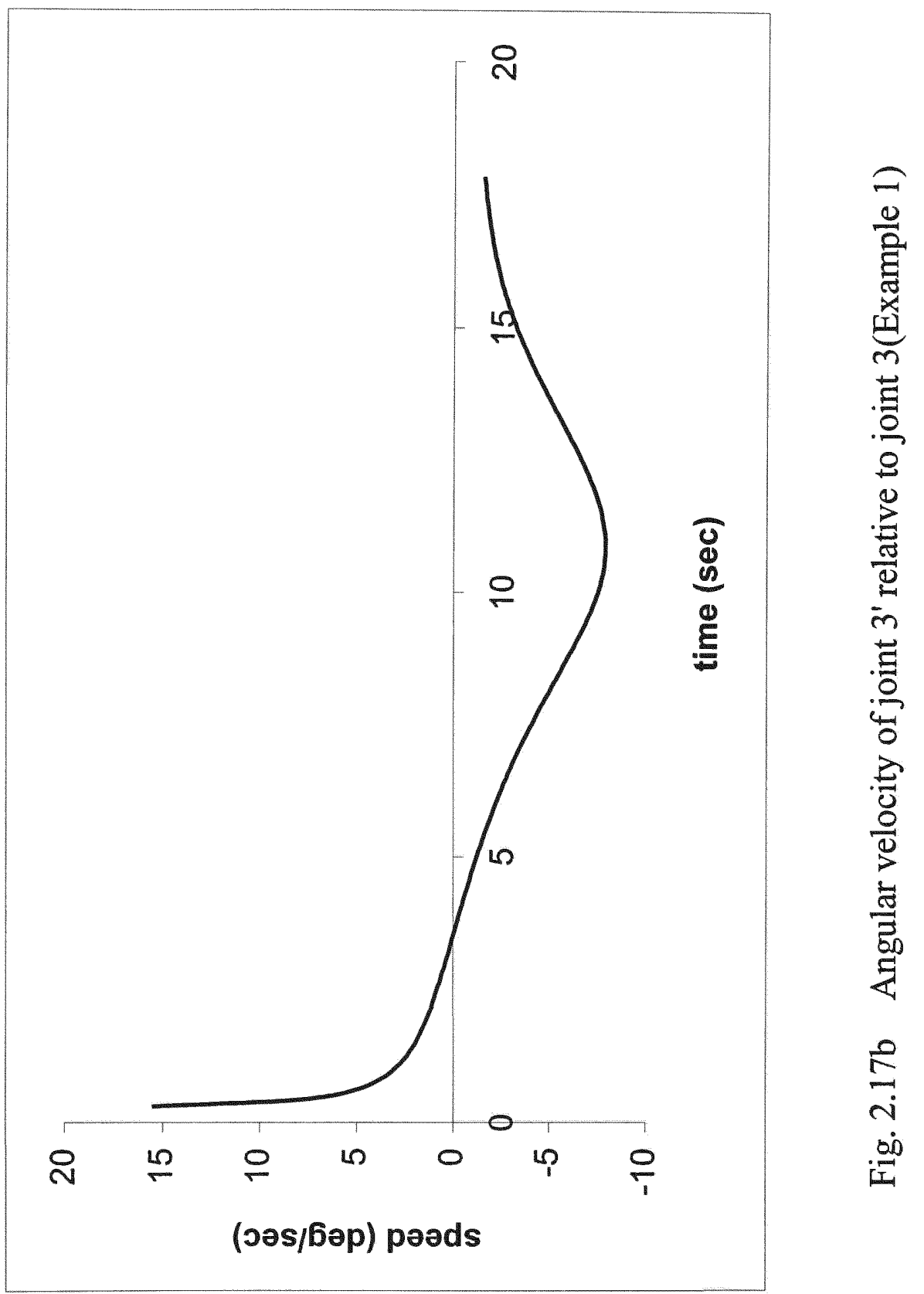




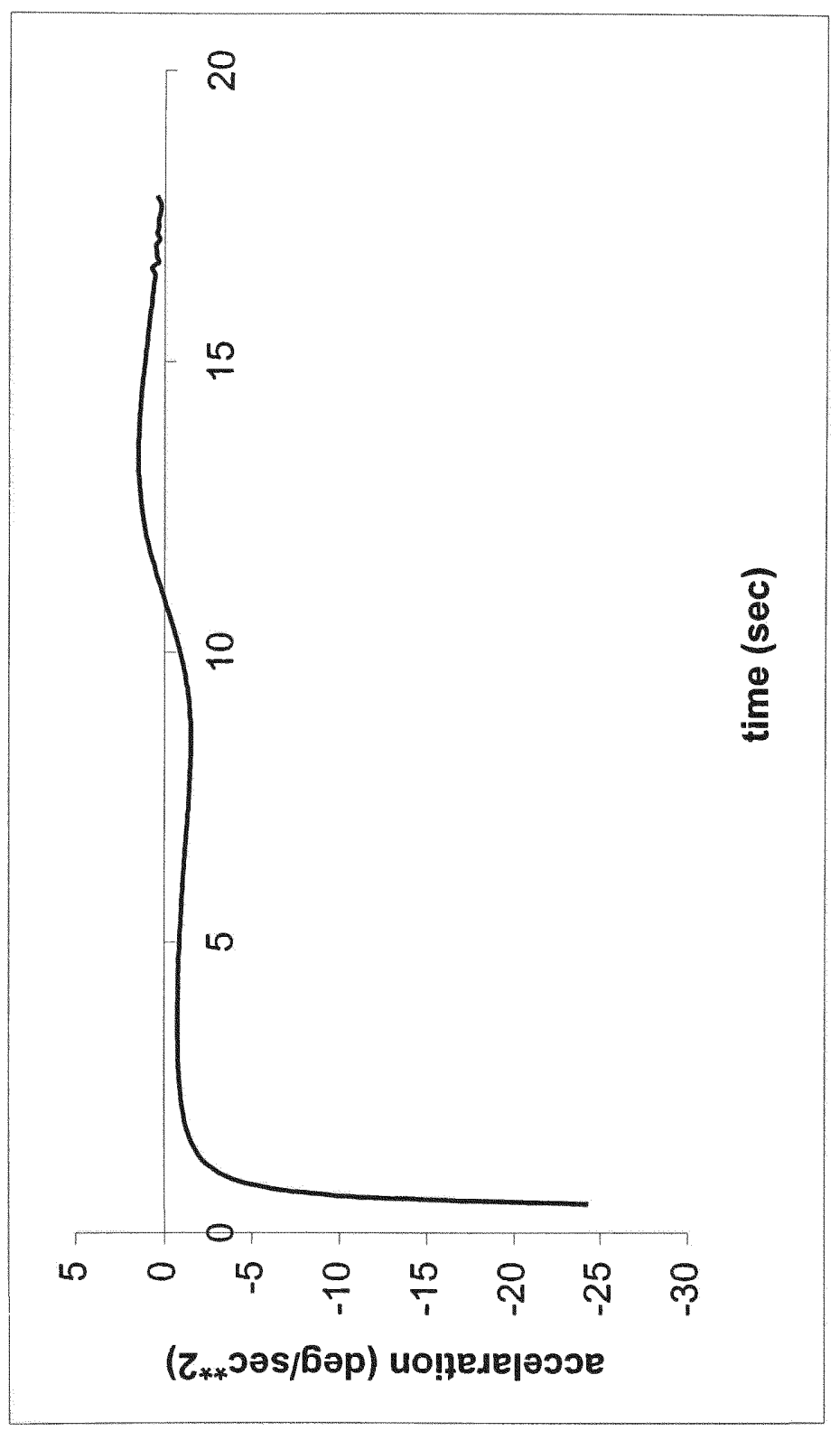

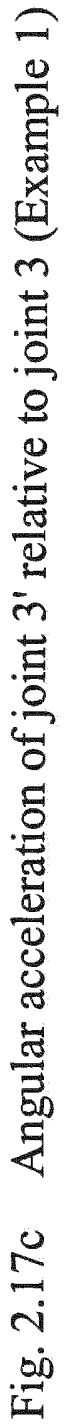




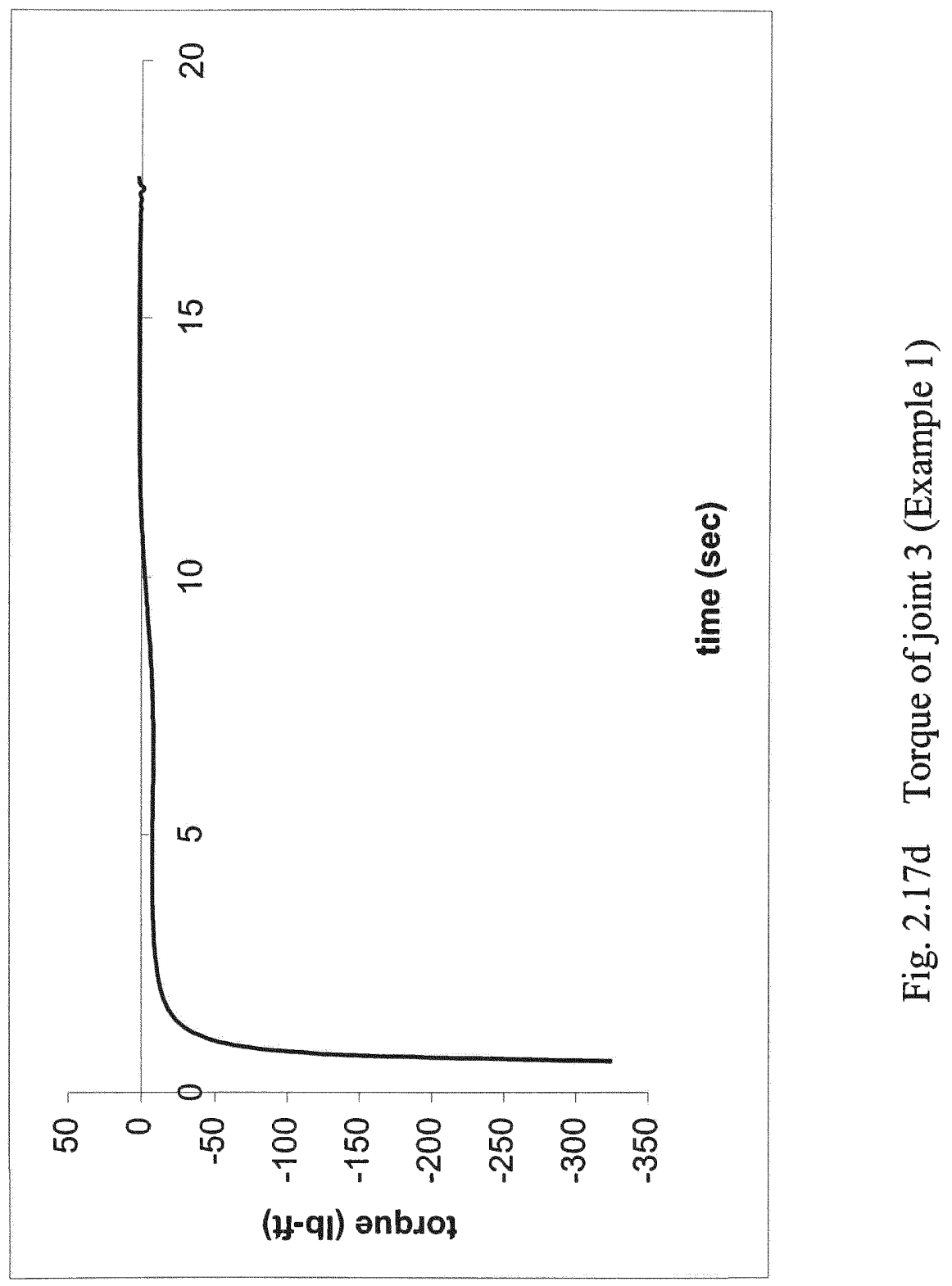




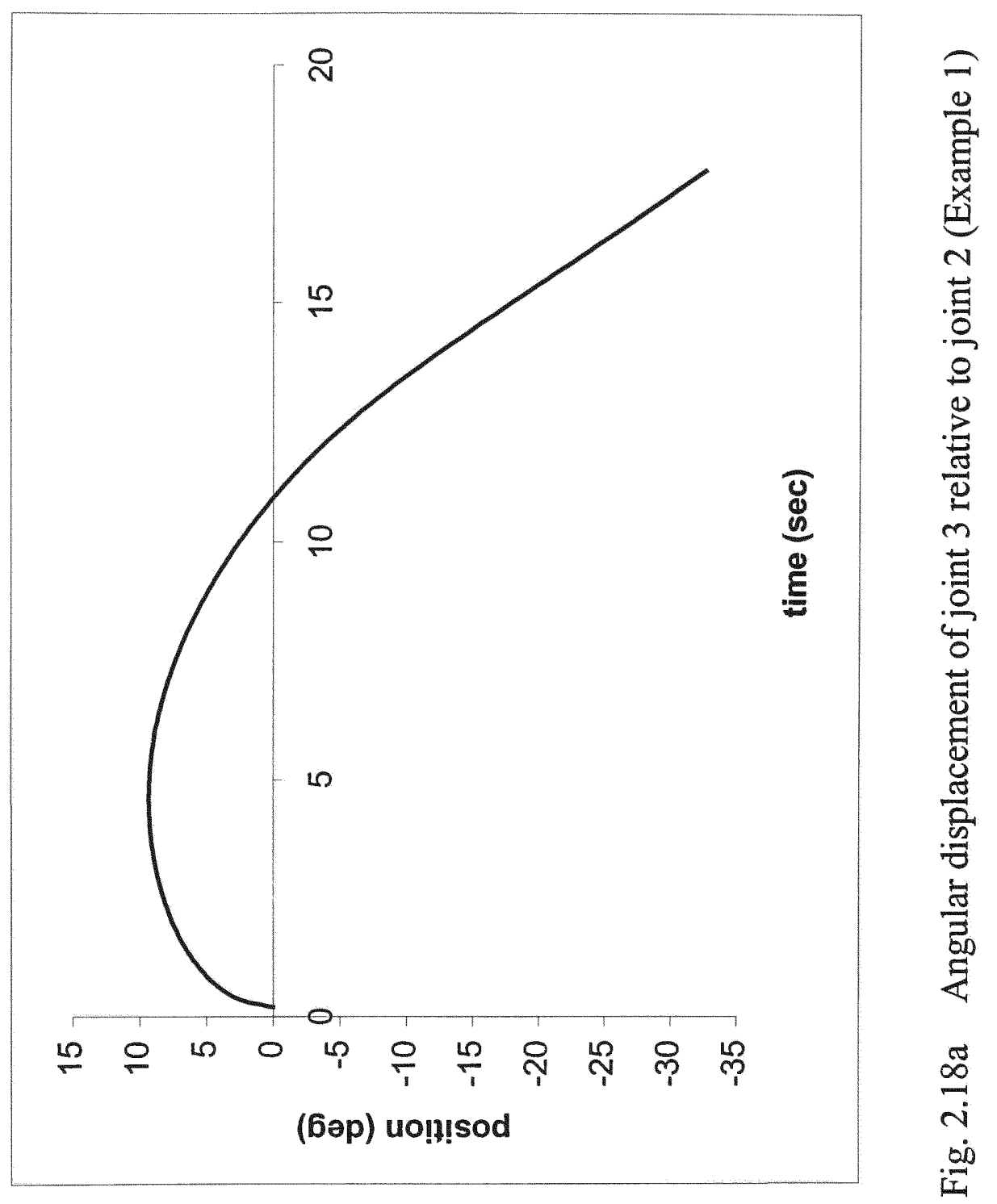




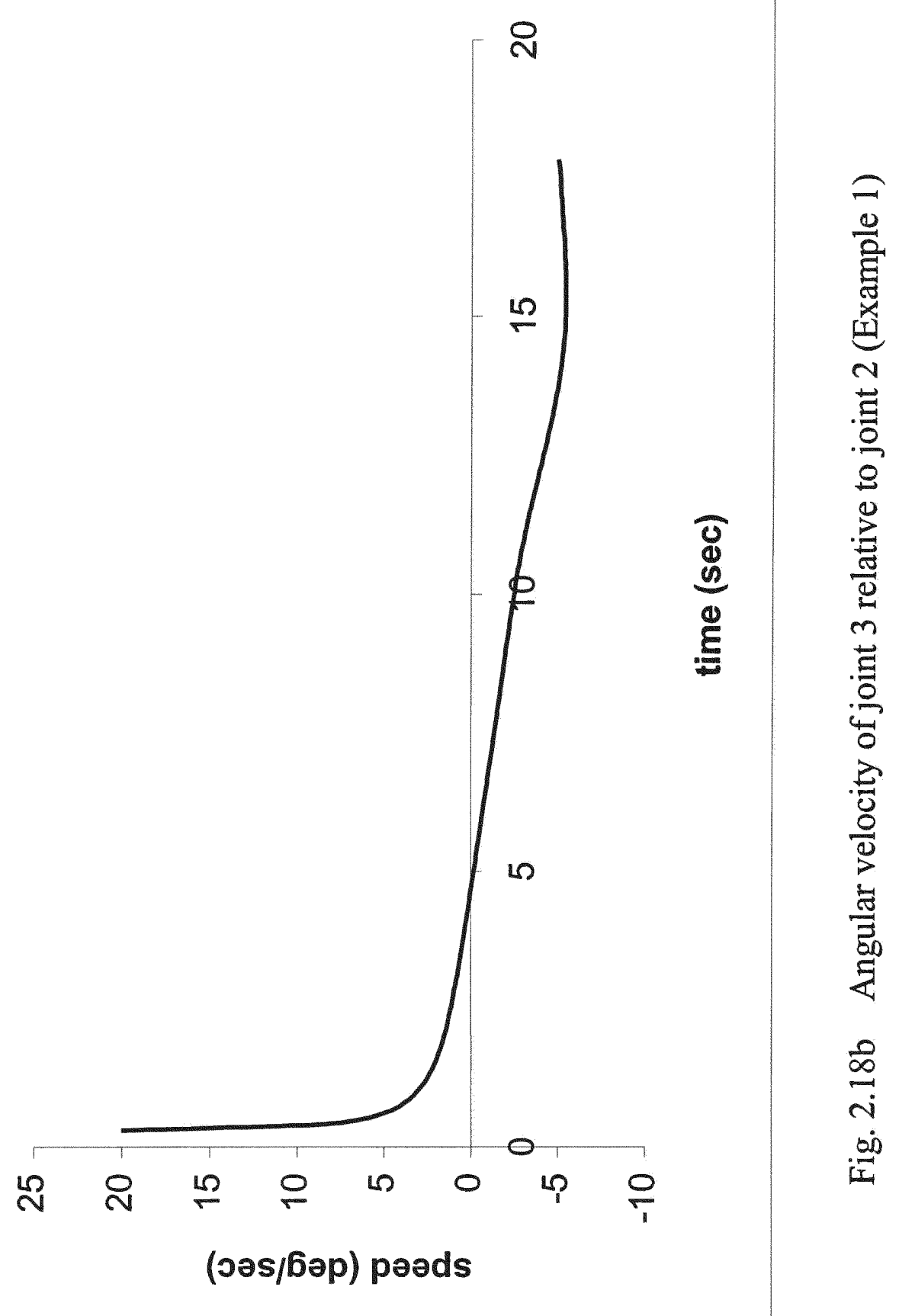




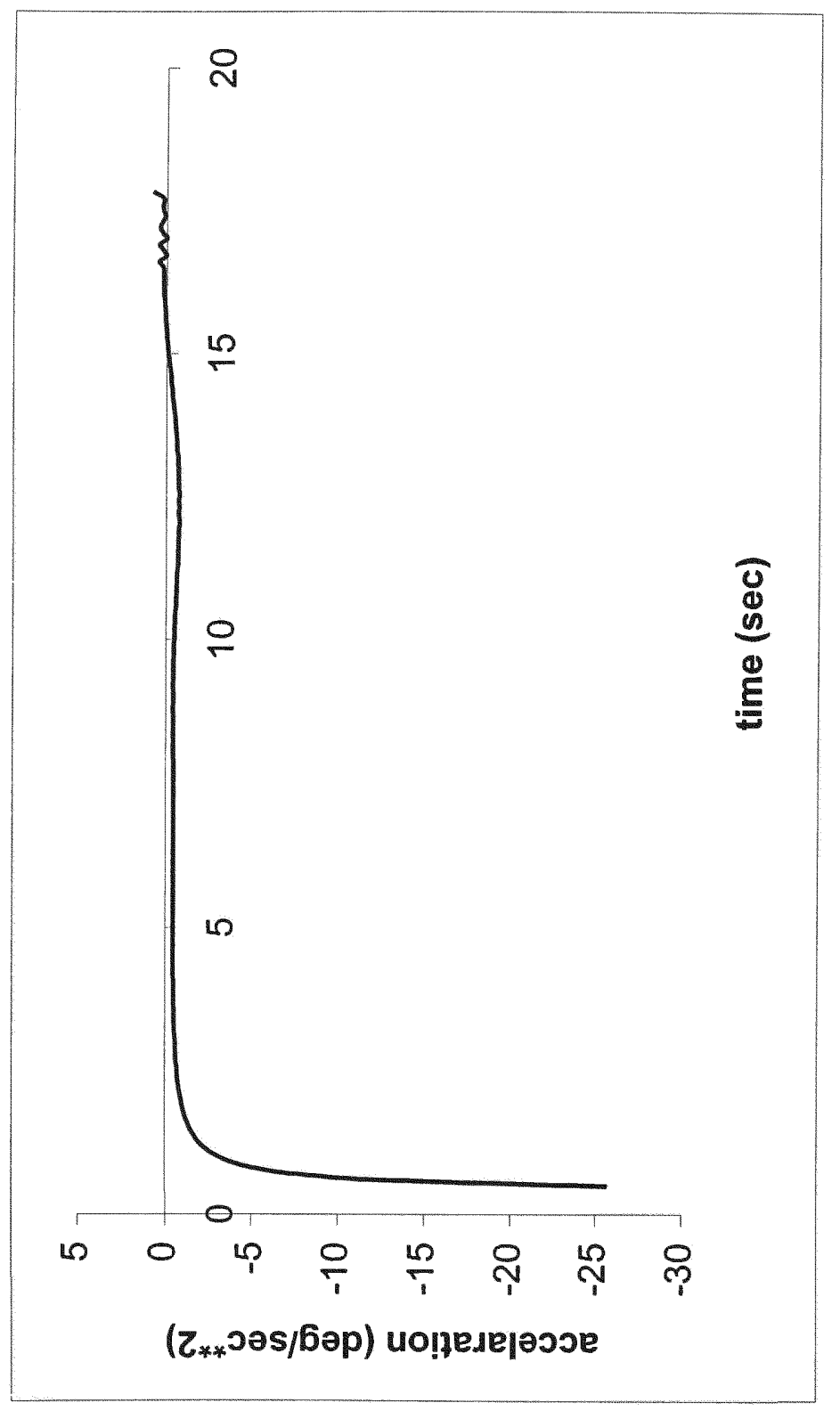

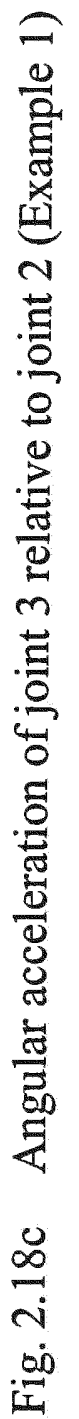




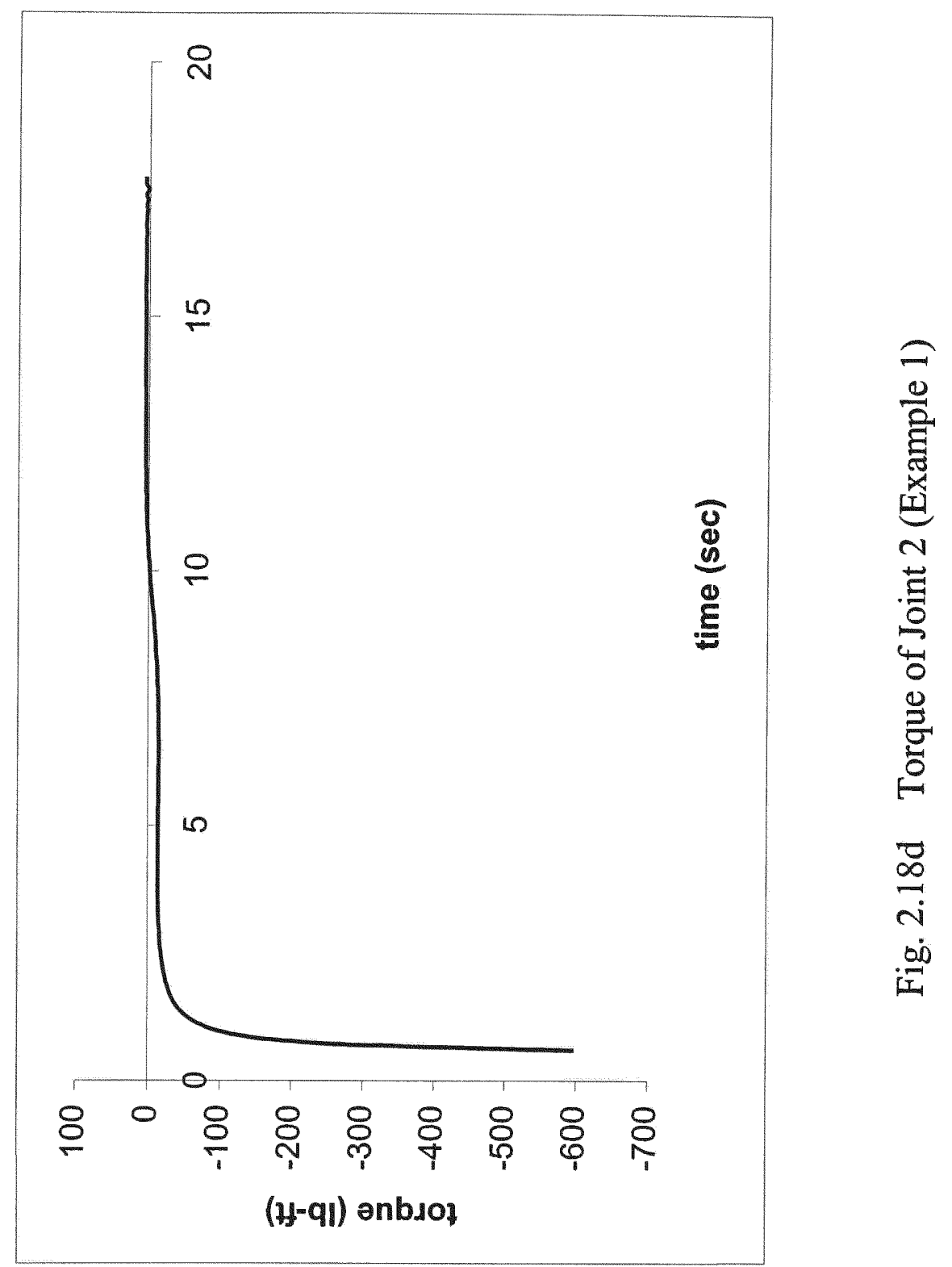


Example 2: $\quad$ Gripper 2 grasps the structural member in the tank and gripper 1 moves from $\mathrm{t} 1$ to $\mathrm{t} 2$ along a straight line trajectory.

Data of angular displacement, velocity and acceleration, and joint torque have been collected from Tele/IGRIP through simulated results as shown in Figs. $2.12 \mathrm{a}$ and $\mathrm{b}$. The end-effector (gripper 1) takes 18 seconds to move from its initial position t1 to final position $\mathrm{t} 2$ while moving in a steady speed of 20 inches per second and carrying a payload of $110 \mathrm{lb}$. Angular displacement of gripper 1with respect to joint 2 is shown in Fig. 2.19a. It increases in magnitude for the first half of its path and then changes direction with magnitude decreased till its final position has been reached. Fig. 2.19b shows the angular velocity of the gripper 1 . It slows down rapidly within the first 1.5 seconds and gradually increases after 9.5 seconds until gripper 1 reaches the maximum angular velocity of $10 \mathrm{deg} / \mathrm{sec}$. Angular acceleration of gripper 1, Fig. 2.19c, also decelerates rapidly in 1.25 seconds and then decreases gradually till it reaches the minimum value of zero degree per second square at the end of 18 seconds. Torque of joint 2 decreases in the first 1.5 seconds and then fluctuates between -2 and $0.75 \mathrm{lb}$ - $\mathrm{ft}$ as shown in Fig. 2.19d. In a similar manner, Figs. 2.20a to d, Figs. 2.21a to d, and Figs. 2.22 a to $\mathrm{d}$ can be analyzed for joints $3,3^{\prime}$ and $2^{\prime}$ respectively. Gripper 2 produces a torque of the same magnitude as joint 2', Fig. 2.22d, on the structural member. 


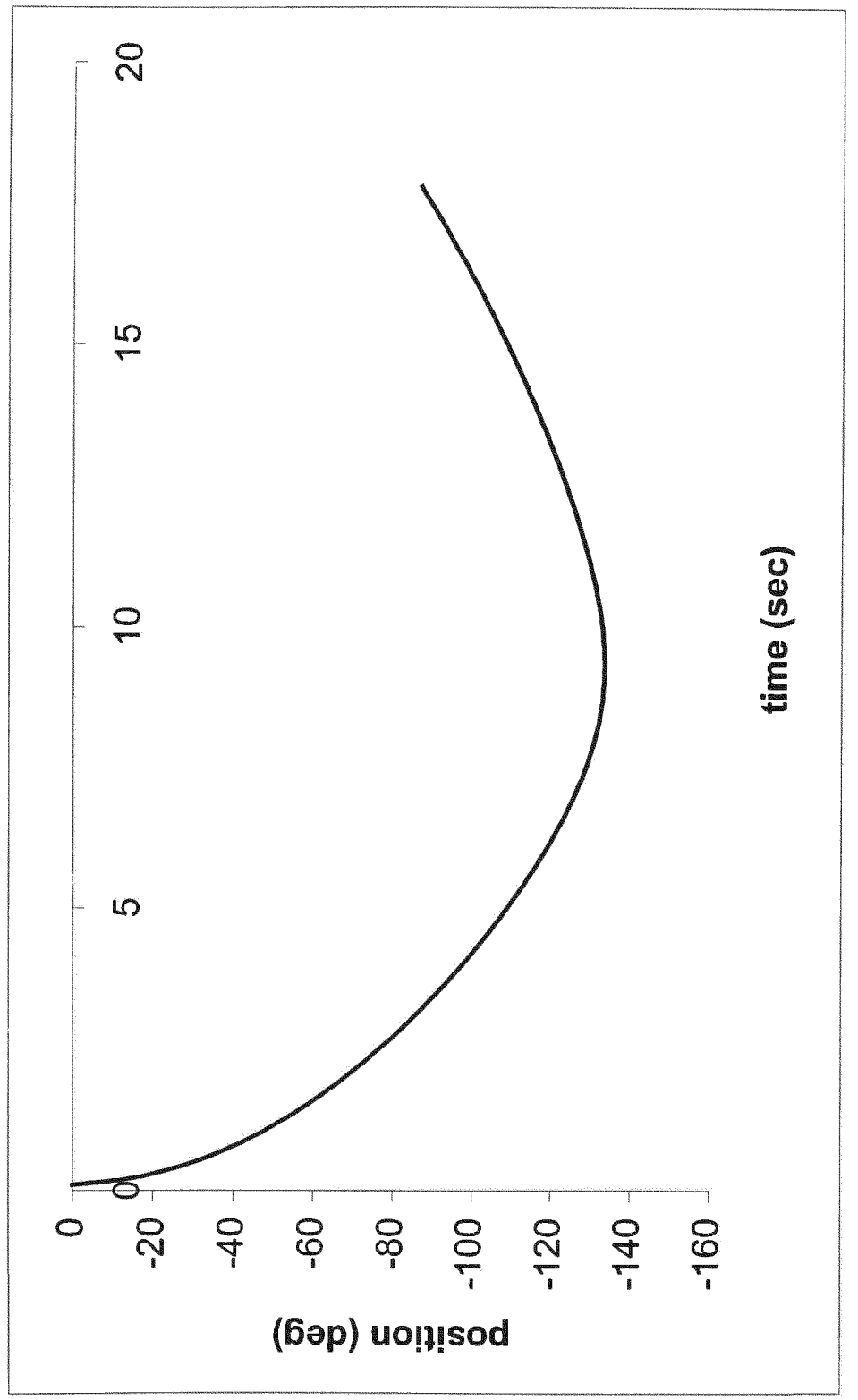

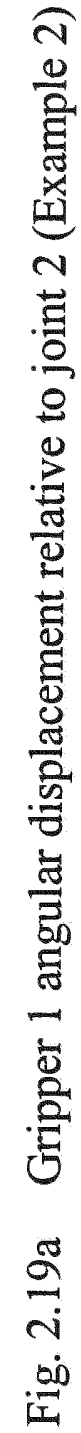




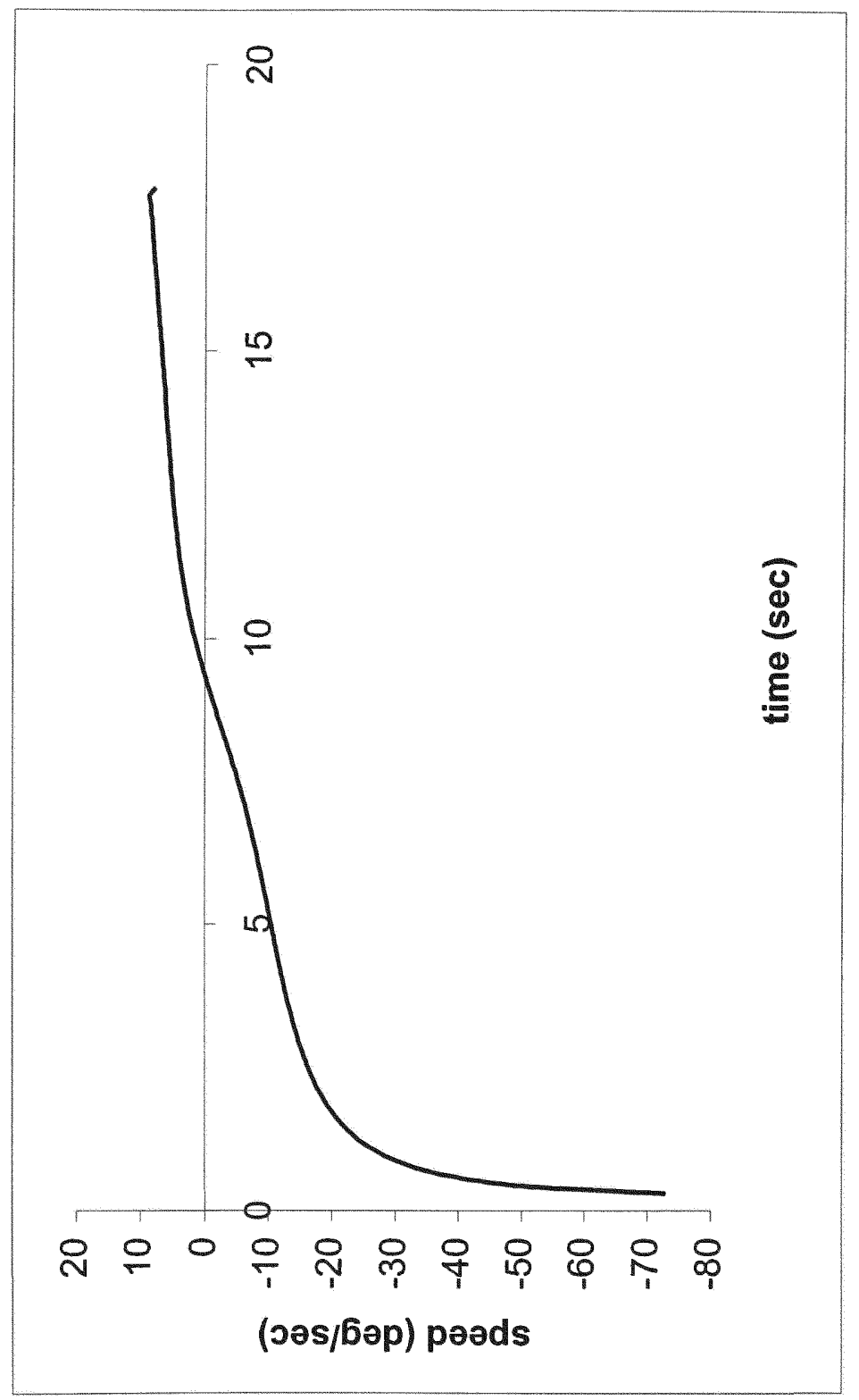

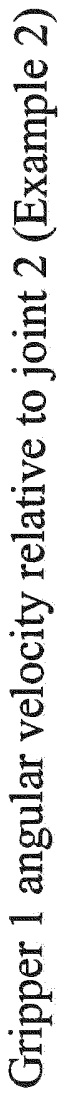

命 


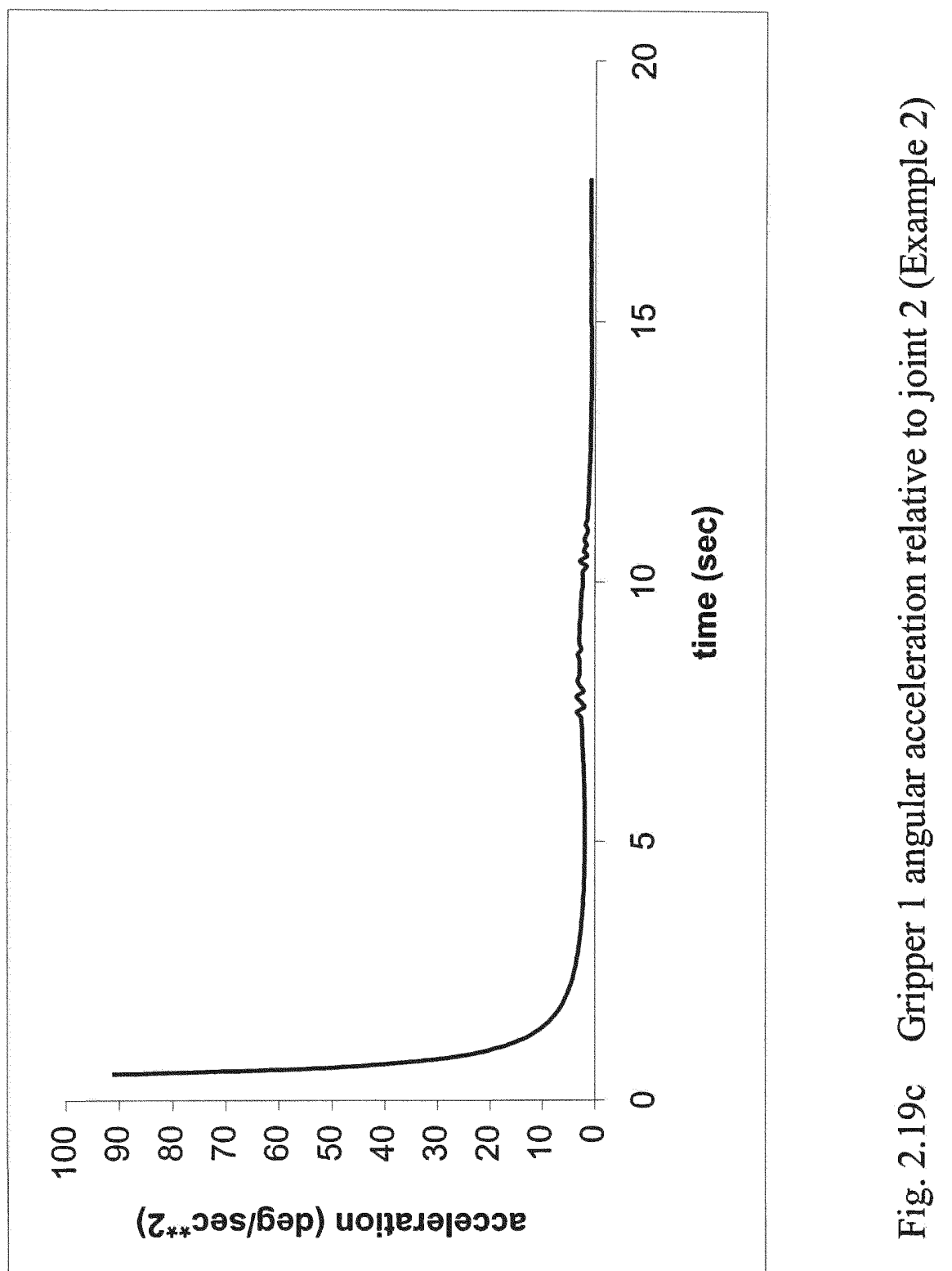




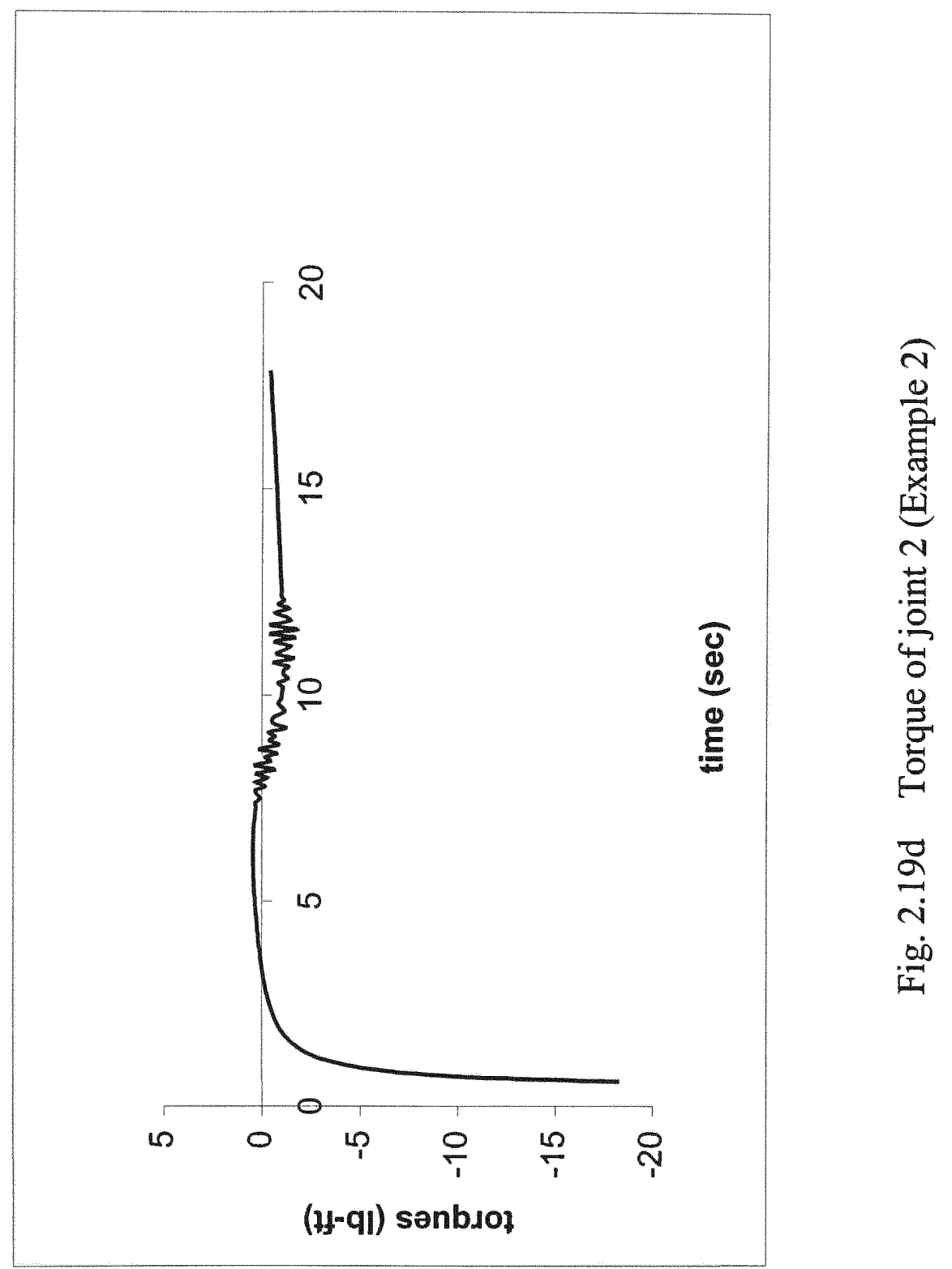




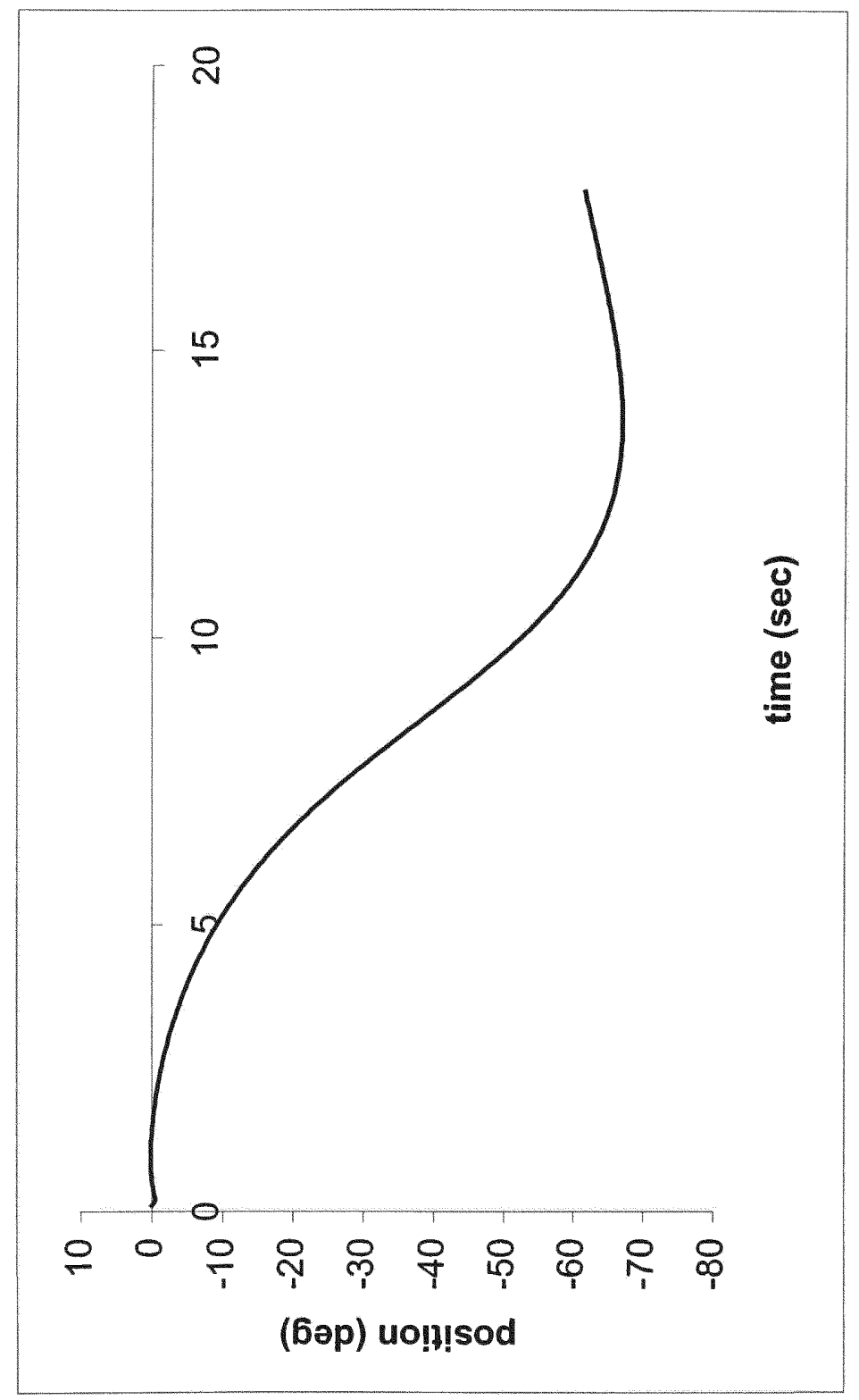

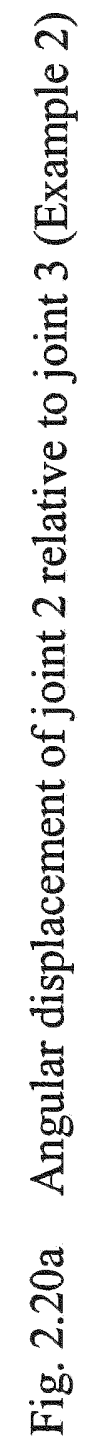




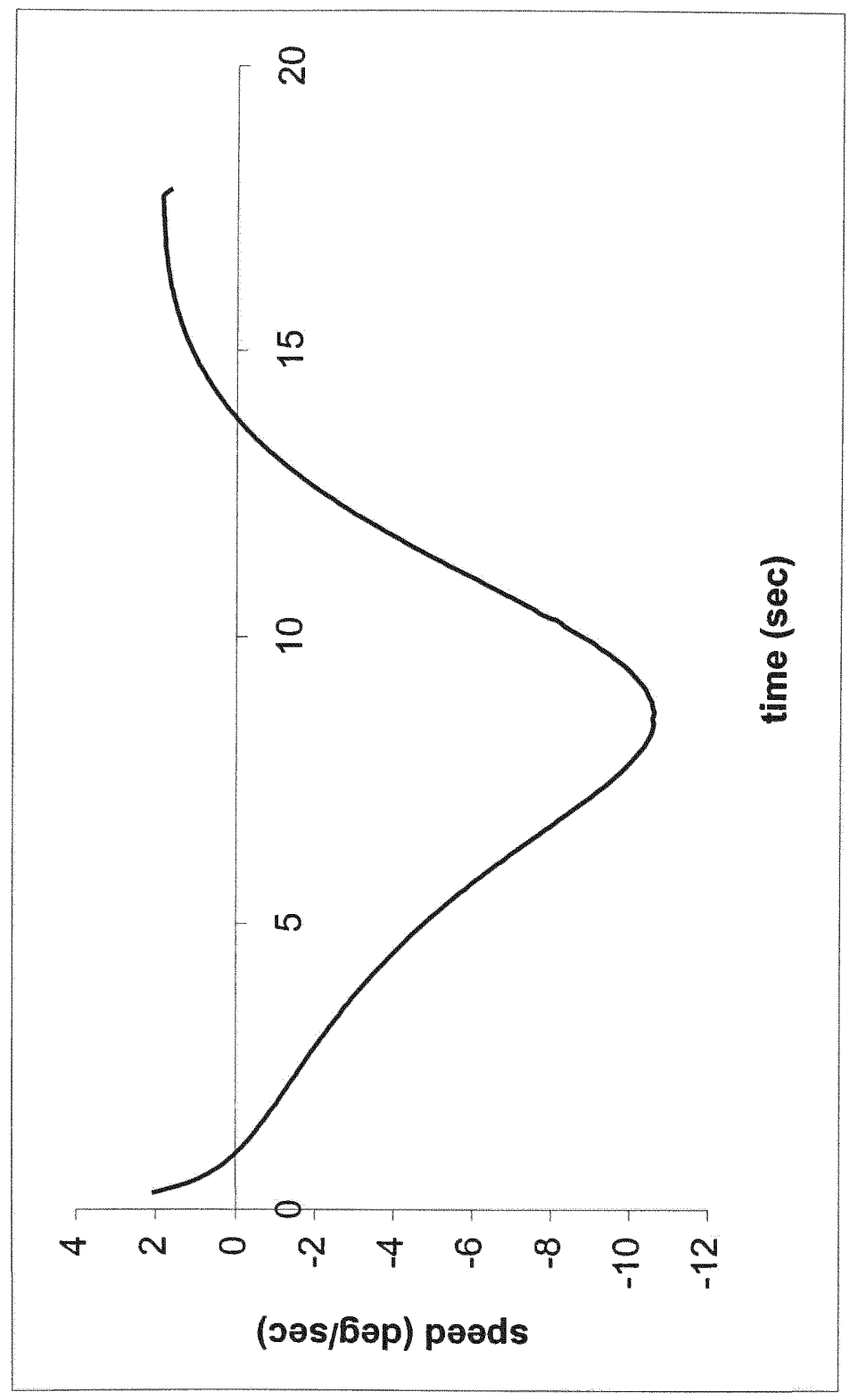

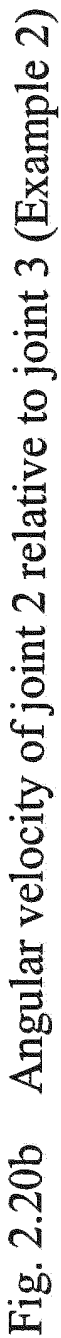




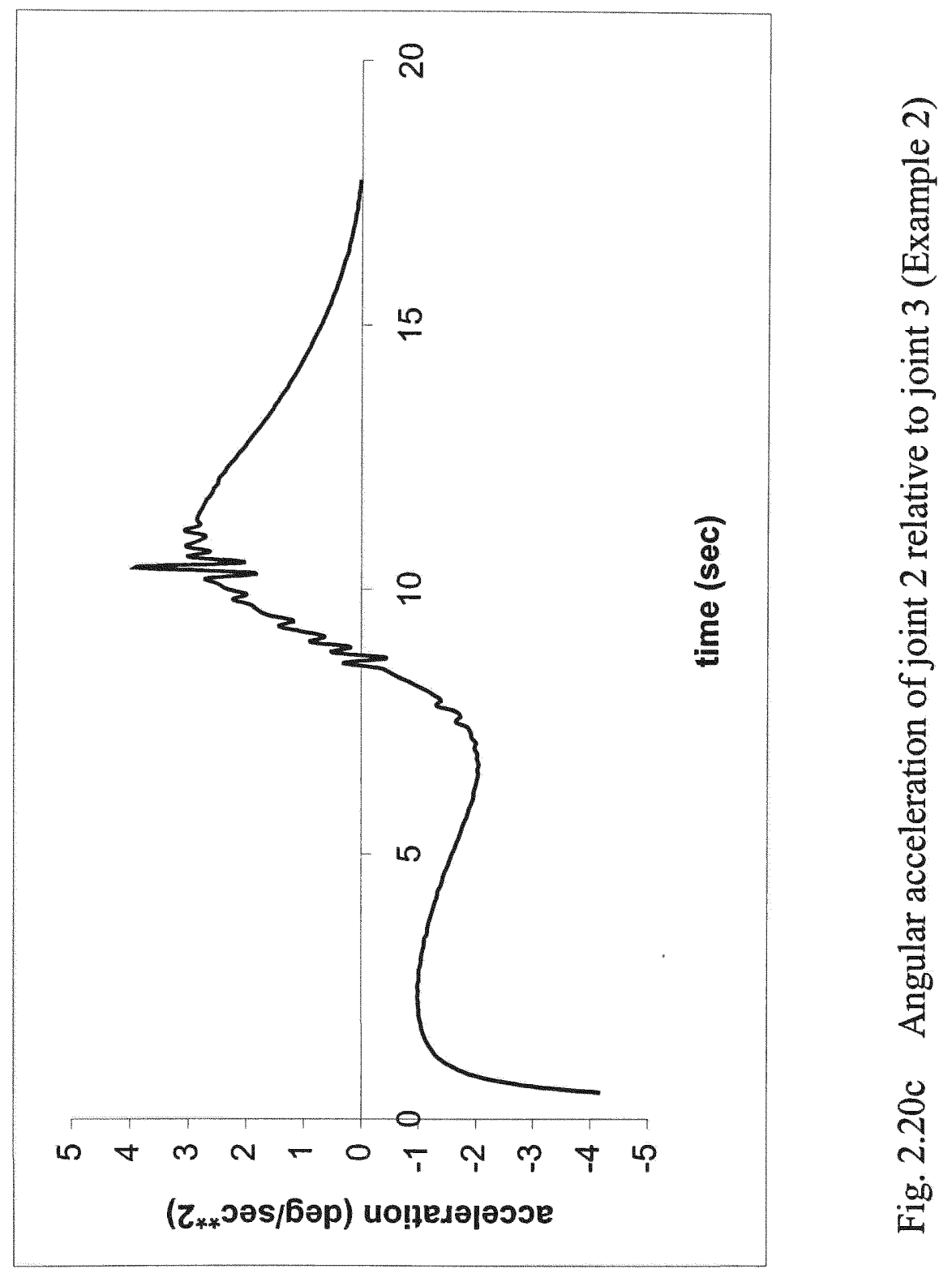




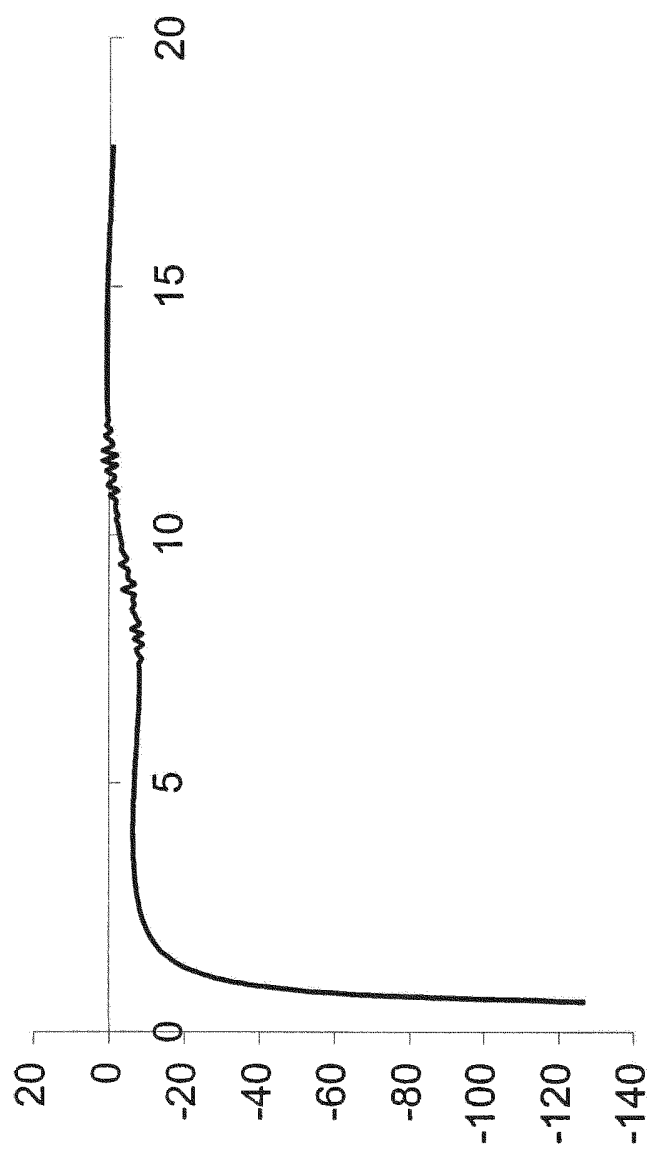

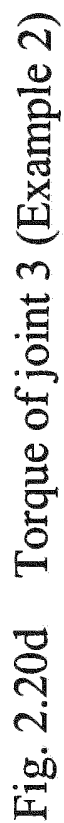

(H-qI) senbsol 


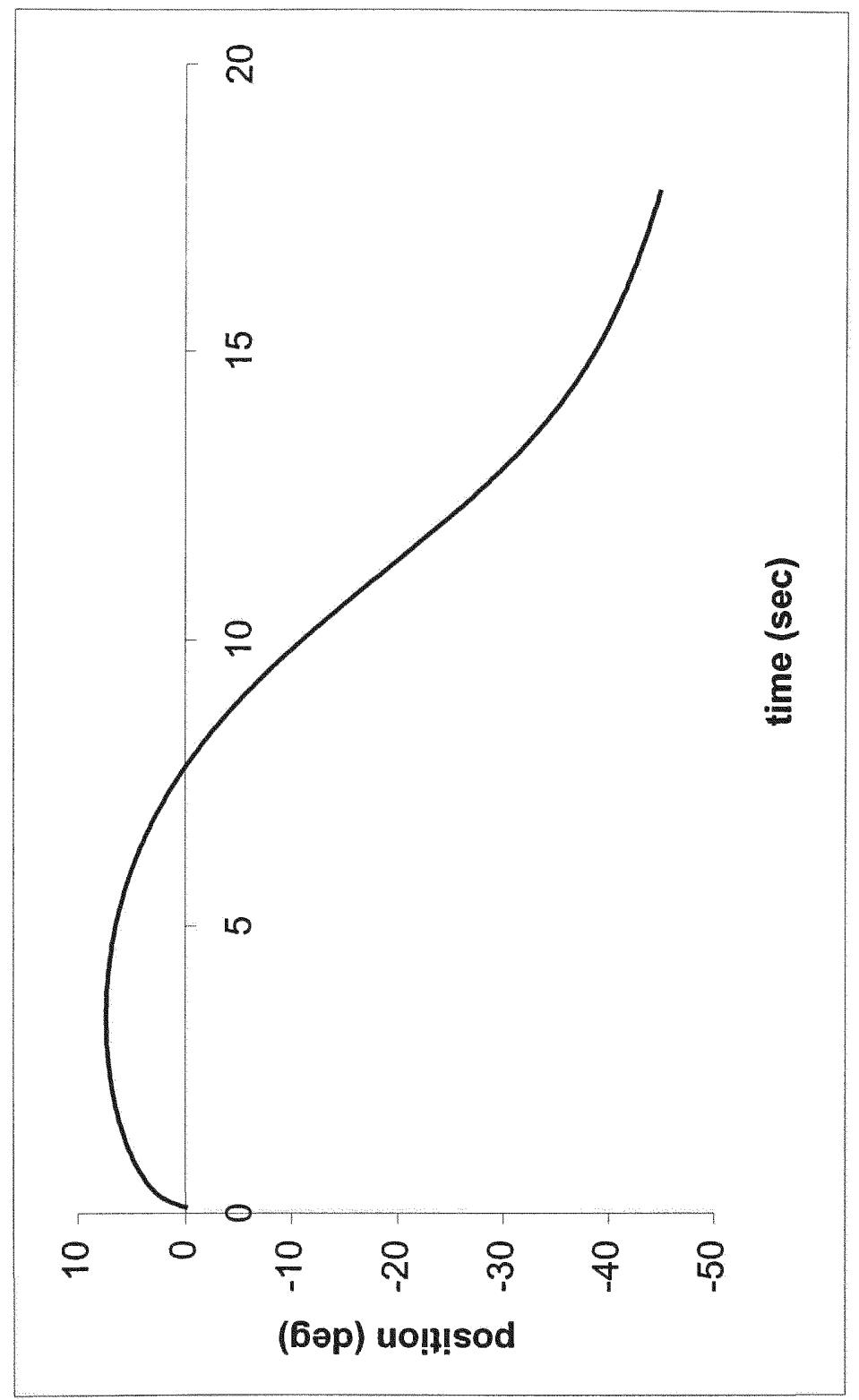

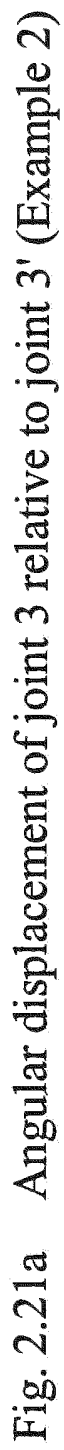




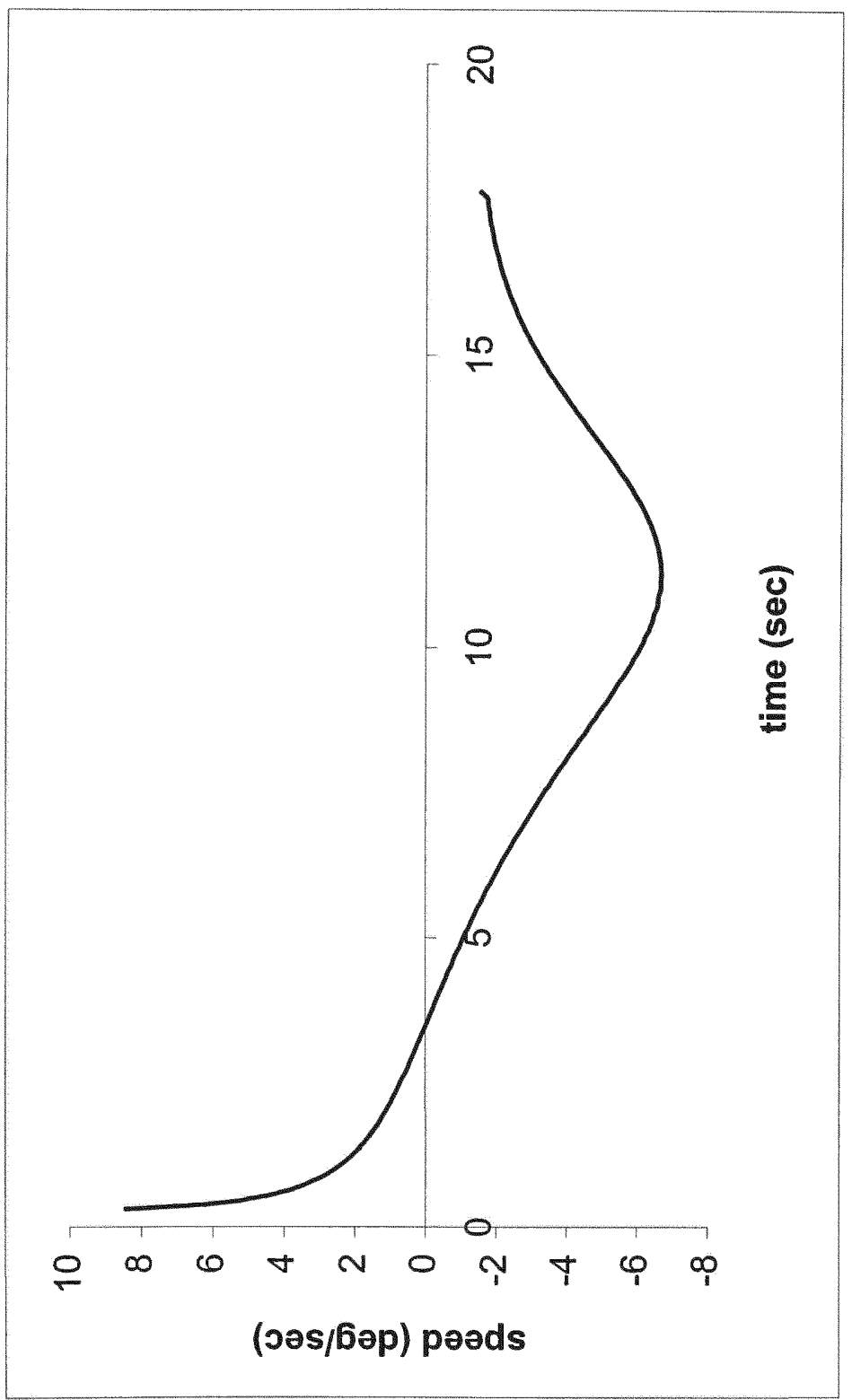

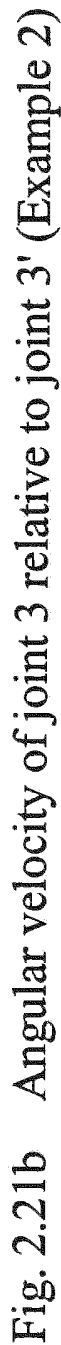




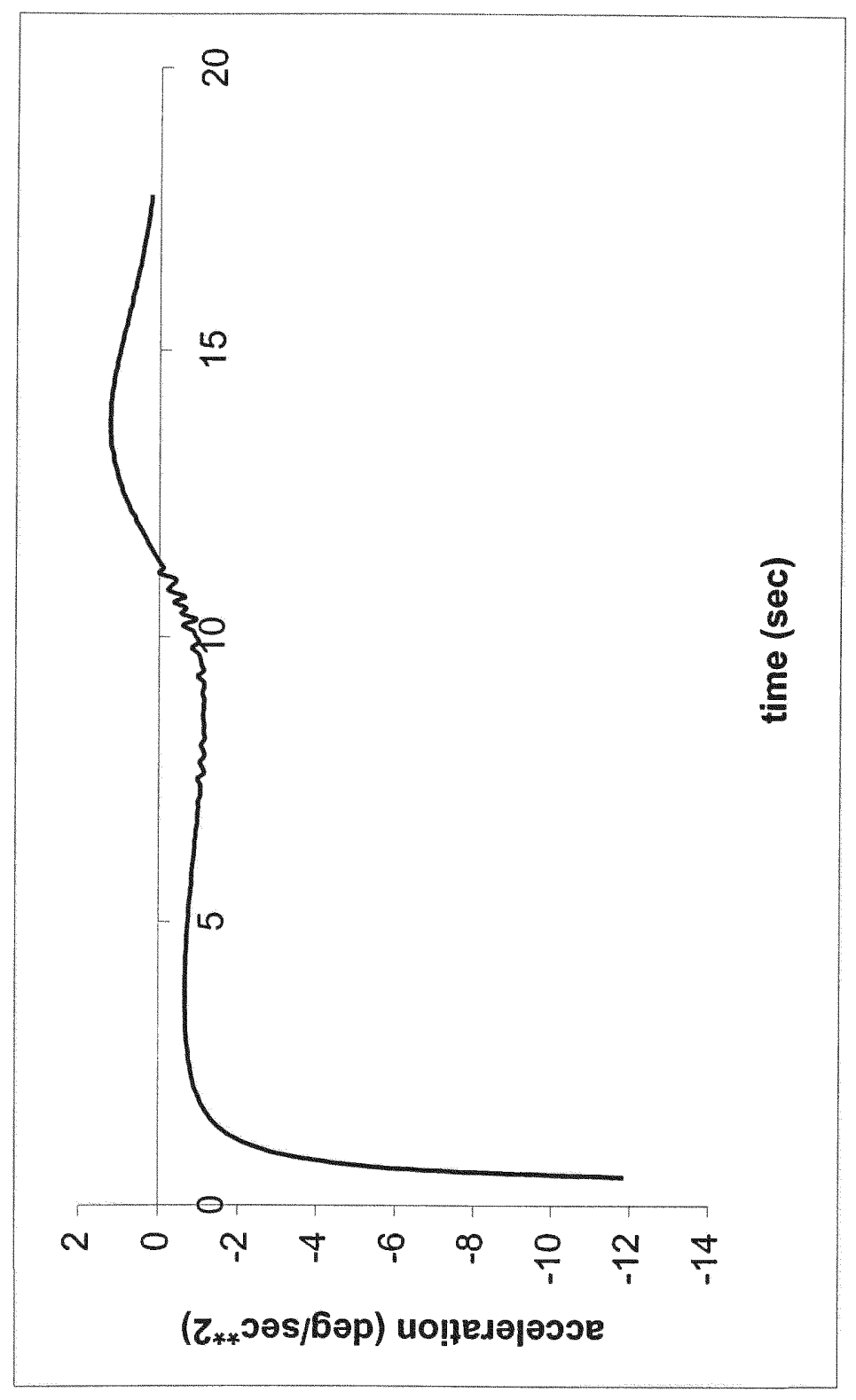

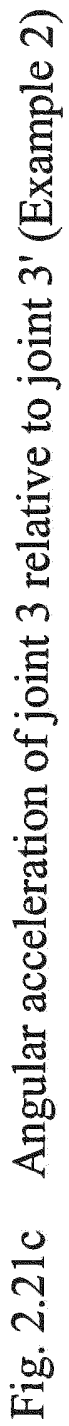




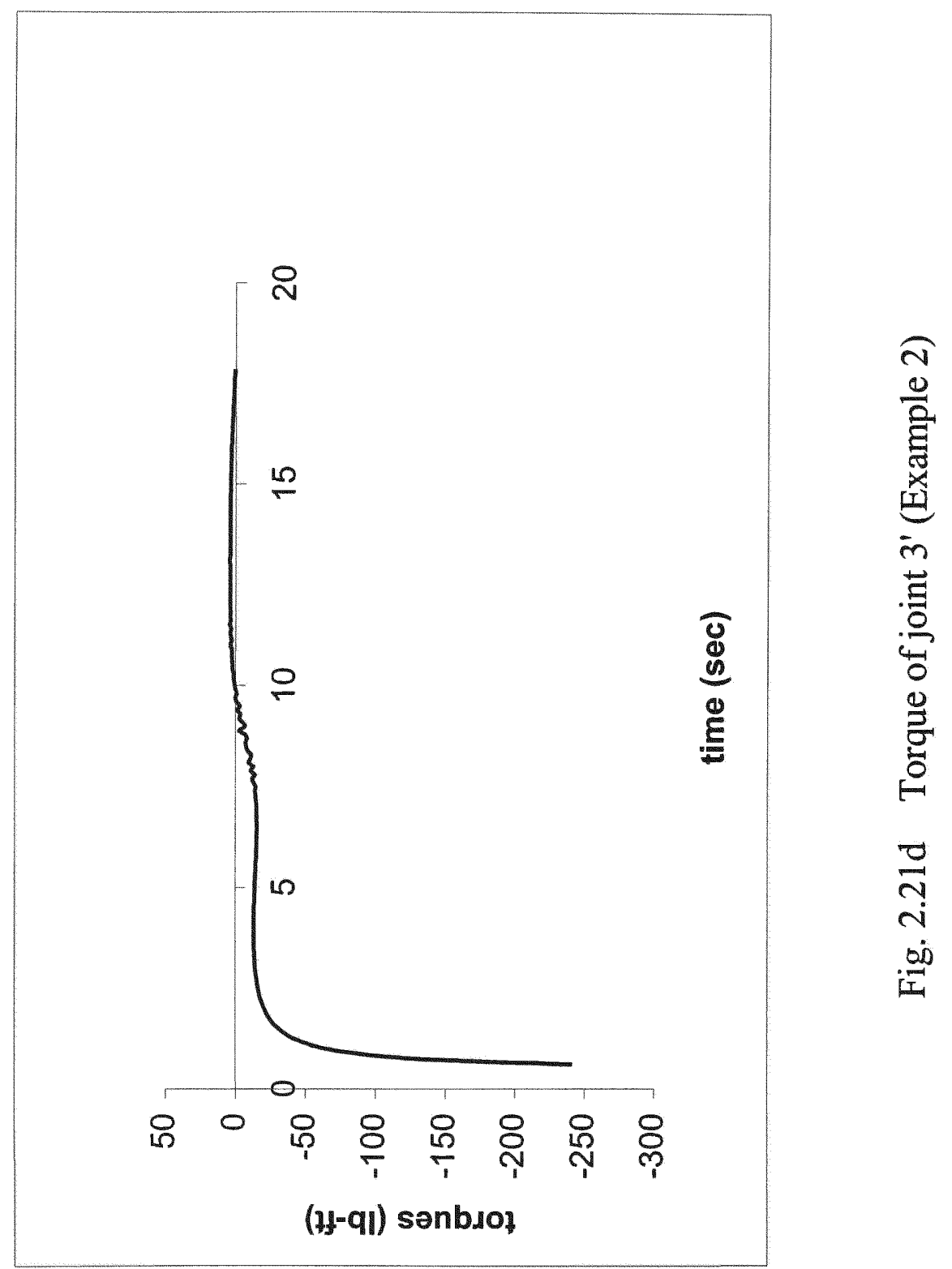




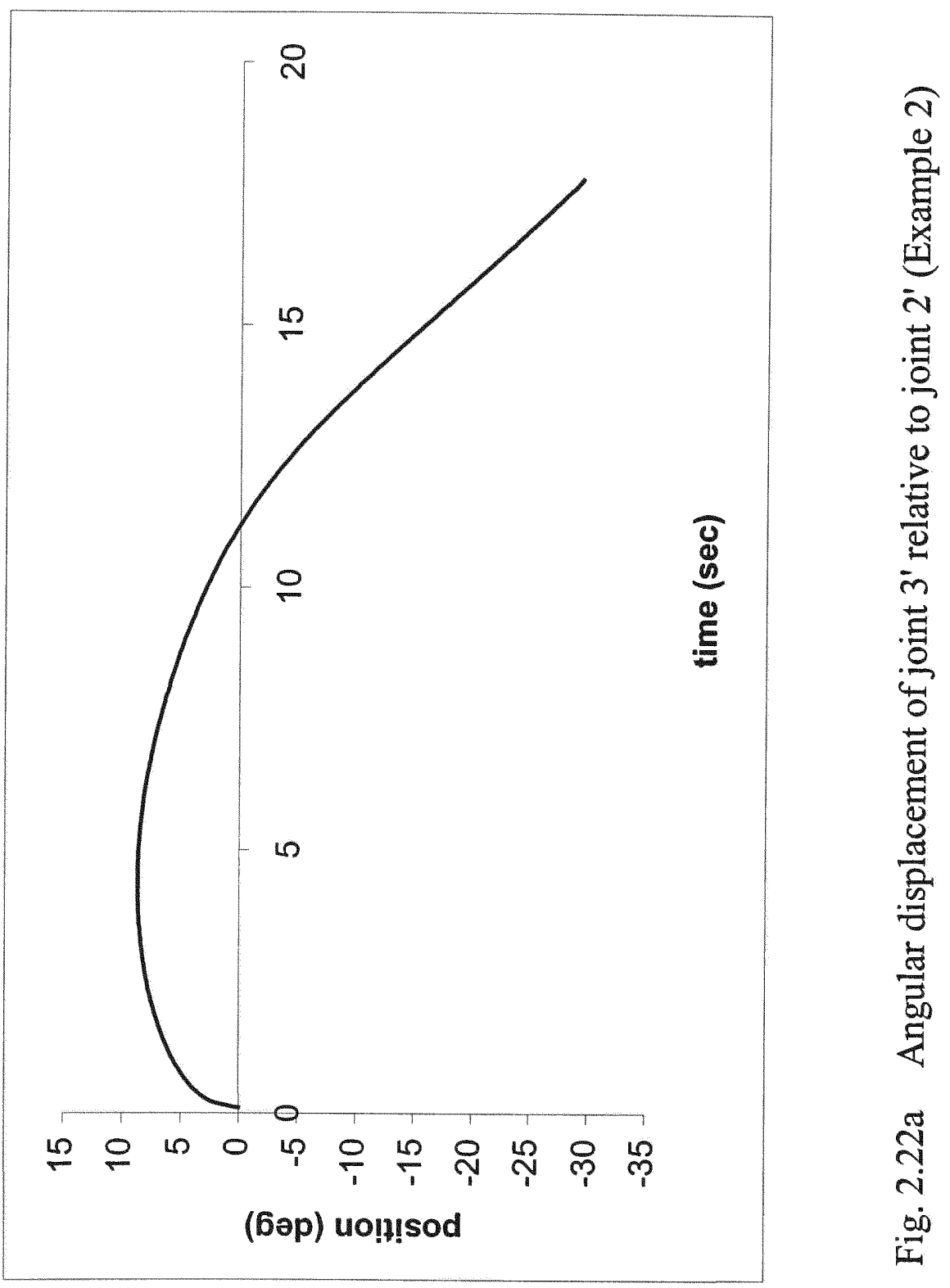




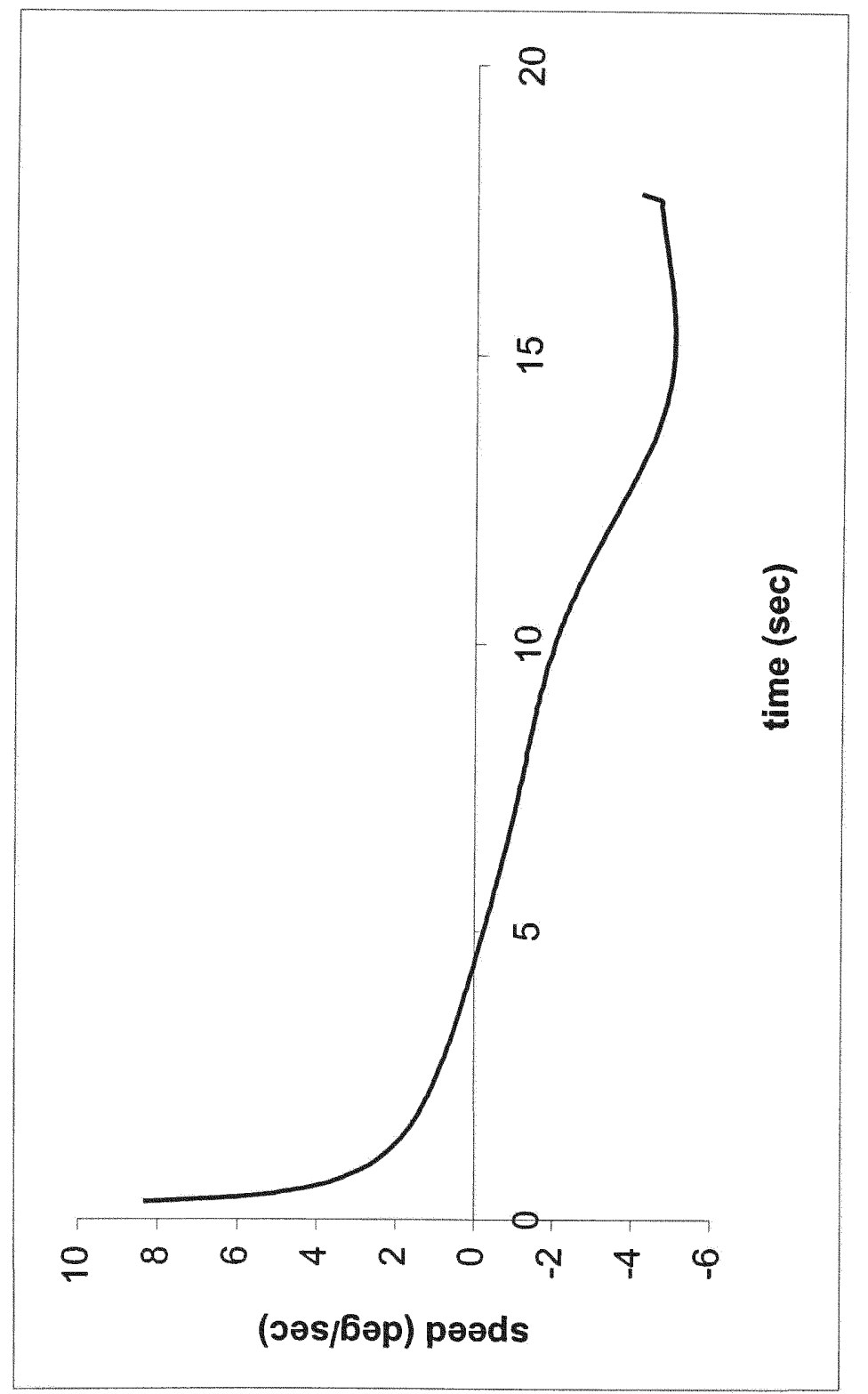

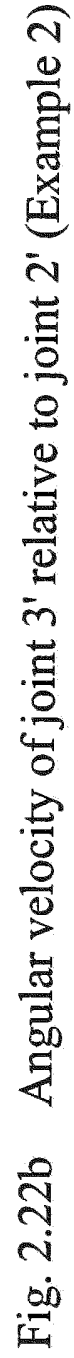




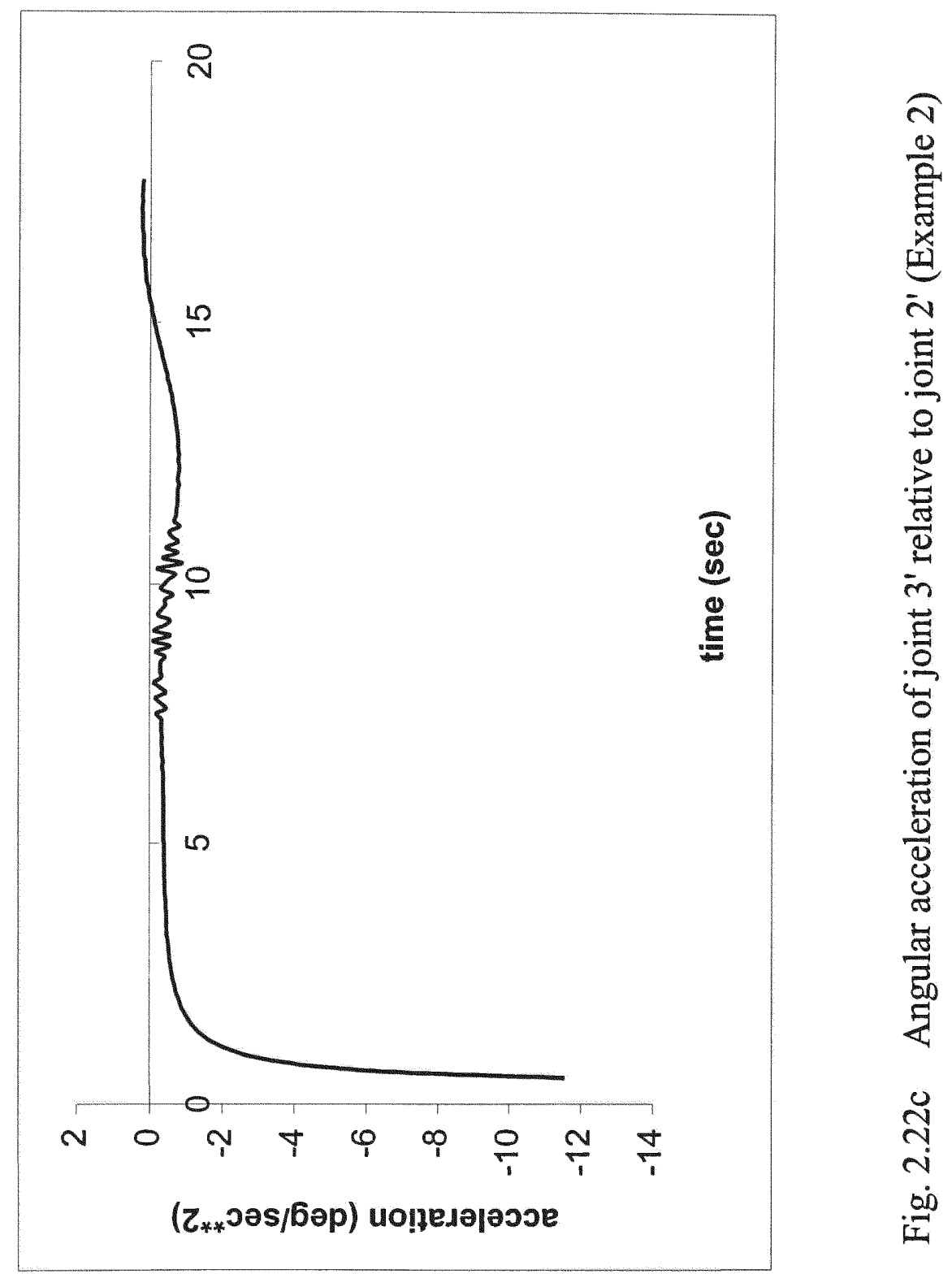




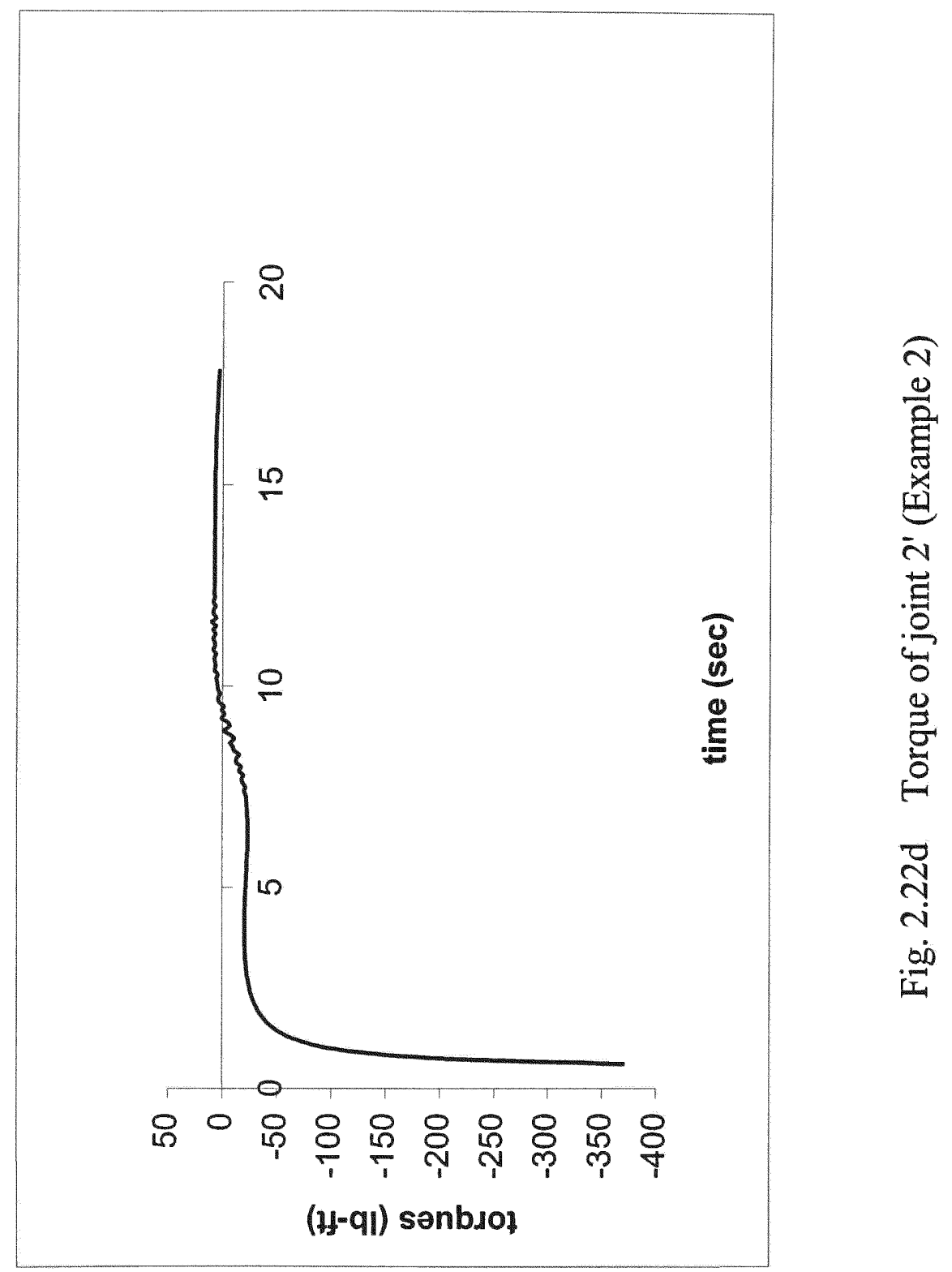


Example 3: Gripper 1 grasps the structural member in the tank and gripper 2 moves from $t 1$ to 5 to grasp the same structural member.

As simulated in Figs. 2.13a to e, both kinematic and dynamic data - angular displacement, velocity, acceleration, and joint torque - for each joint have been collected in Tele/IGRIP. The end-effector (gripper 2) of the RTD system is moving with a steady speed of 20 inches per second and carrying a payload of $110 \mathrm{lb}$. In addition, gripper 2 moves from its initial position $\mathrm{t} 1$ to the final position $\mathrm{t} 5$ passing through intermediate tag points $t 2, t 3$ and $t 4$, within 23 seconds which is defined automatically by Tele/IGRIP. Tele/IGRIP collects the data with a time interval of 0.1 seconds $(\Delta t=0.1 \mathrm{sec})$. Gripper 2 follows a straight line trajectory between two adjacent tag points. Each joint kinematic data are divided into four figures for four trajectory segments from $t 1$ to $t 2, t 2$ to $t 3, t 3$ to $\mathrm{t} 4$, and $\mathrm{t} 4$ to $\mathrm{t} 5$.

Graphs in Fig. 2.23a show the angular displacement of gripper 2 relative to joint $2^{\prime}$. It gradually increases in magnitude and never exceeds the maximum limit $\left( \pm 135^{\circ}\right)$. Angular velocity of gripper 2, Fig. 2.23b, slows down while approaching each of the subsequent point in the trajectory segment. It is observed that discontinuity of the curve occurs at some points, which must have been caused due to the small time interval $\Delta t$. Any of such sudden changes in the curve can be neglected, and the data between discontinuous points should be interpolated by connecting their adjacent points that follow the general trend of the curve. To interpret Fig. $2.23 \mathrm{c}$, it shows that gripper 2 decelerates between each trajectory segment. Discontinuities are also seen in these figures and data should be interpolated between discontinuous points. Torque of joint 2', connected to gripper 2, decreases in magnitude at the end of each trajectory segment. 
Discontinuous points have to be interpolated in this case too. Torques in joint $2^{\prime}$ approximates to zero $\mathrm{lb}-\mathrm{ft}$ between tag points 3 and 4 , and 4 and 5. Similar interpretations can be made for joints 3', 3 and 2 by referring to Figs 2.24 a to d, Figs 2.25 a to d, and Figs 2.27 a to $\mathrm{d}$, respectively.

The VTM elevates gripper 2 from 56.95 " to $66.25^{\prime \prime}$ so that it is able to grasps the same structural member. Fig. 2.26a shows the angular displacement of the VTM. It is obvious that it decreases linearly. The angular velocity, Fig. $2.26 \mathrm{~b}$, of the VTM is constant between trajectory segments since the angular displacements are varied linearly. Angular acceleration of the VTM is represented in Fig. 2.26c. It is almost zero degree per second square throughout the entire path because of constant angular velocity in each trajectory segment. Discontinuous points in the curves must be interpolated as well. Torque associated with the VTM is shown in Fig. 2.26d. The resultant torque on VTM is small, between $-0.5 \mathrm{lb}-\mathrm{ft}$ and $0.025 \mathrm{lb}-\mathrm{ft}$. The torque applied to the structural member, exerted by gripper 1, is equal to the torque at joint 2, Fig. $2.27 \mathrm{~d}$. 

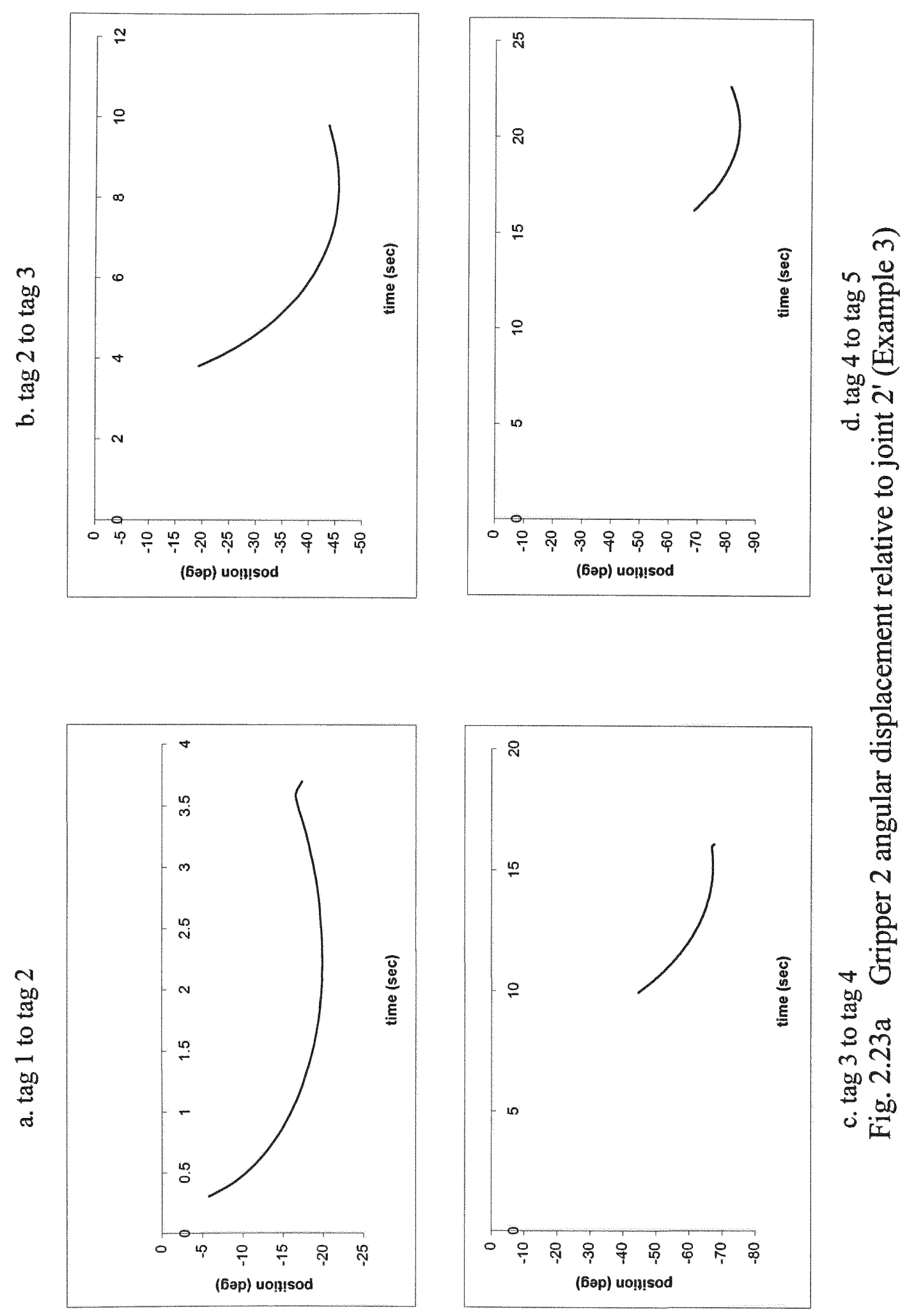

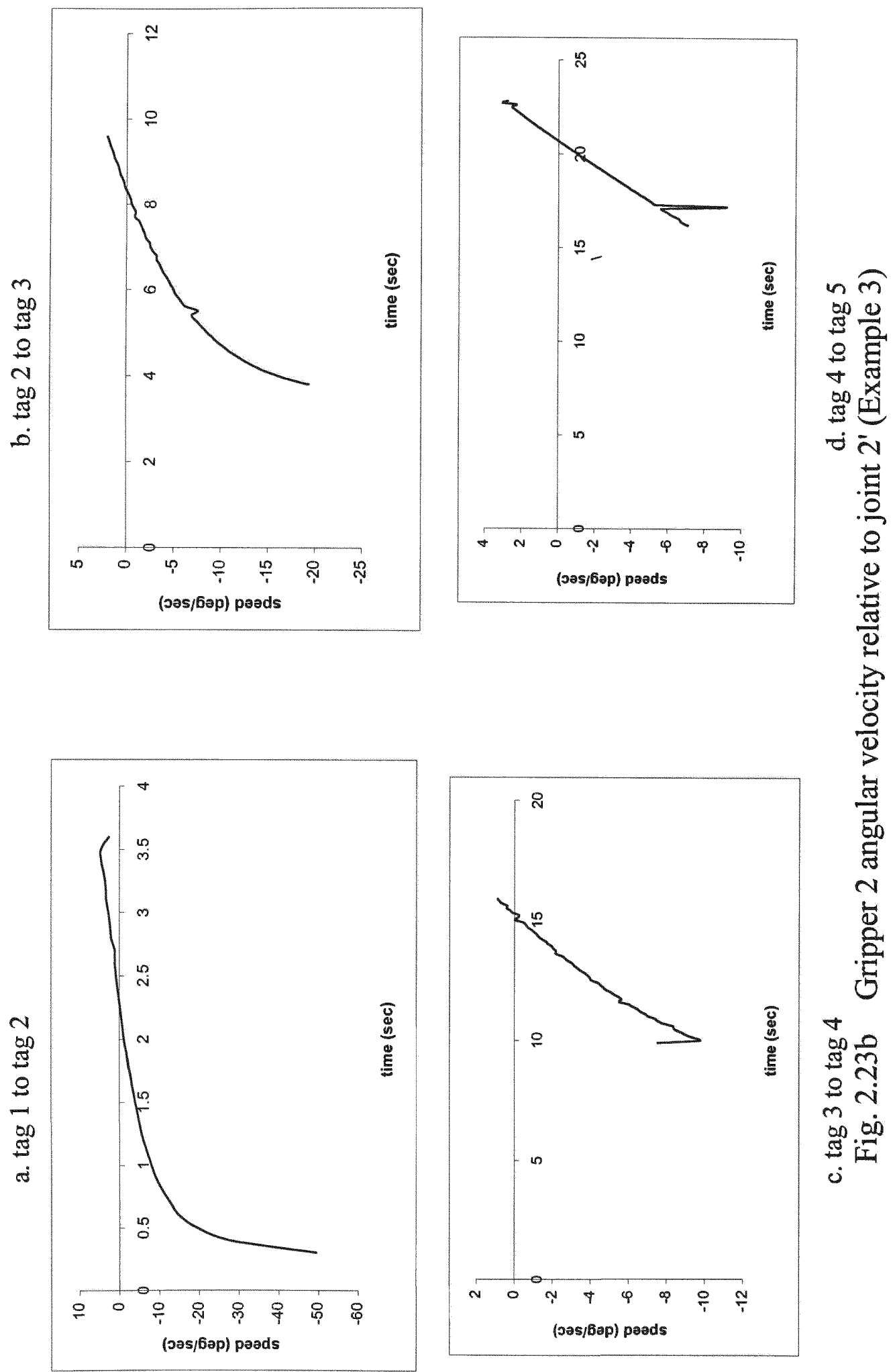

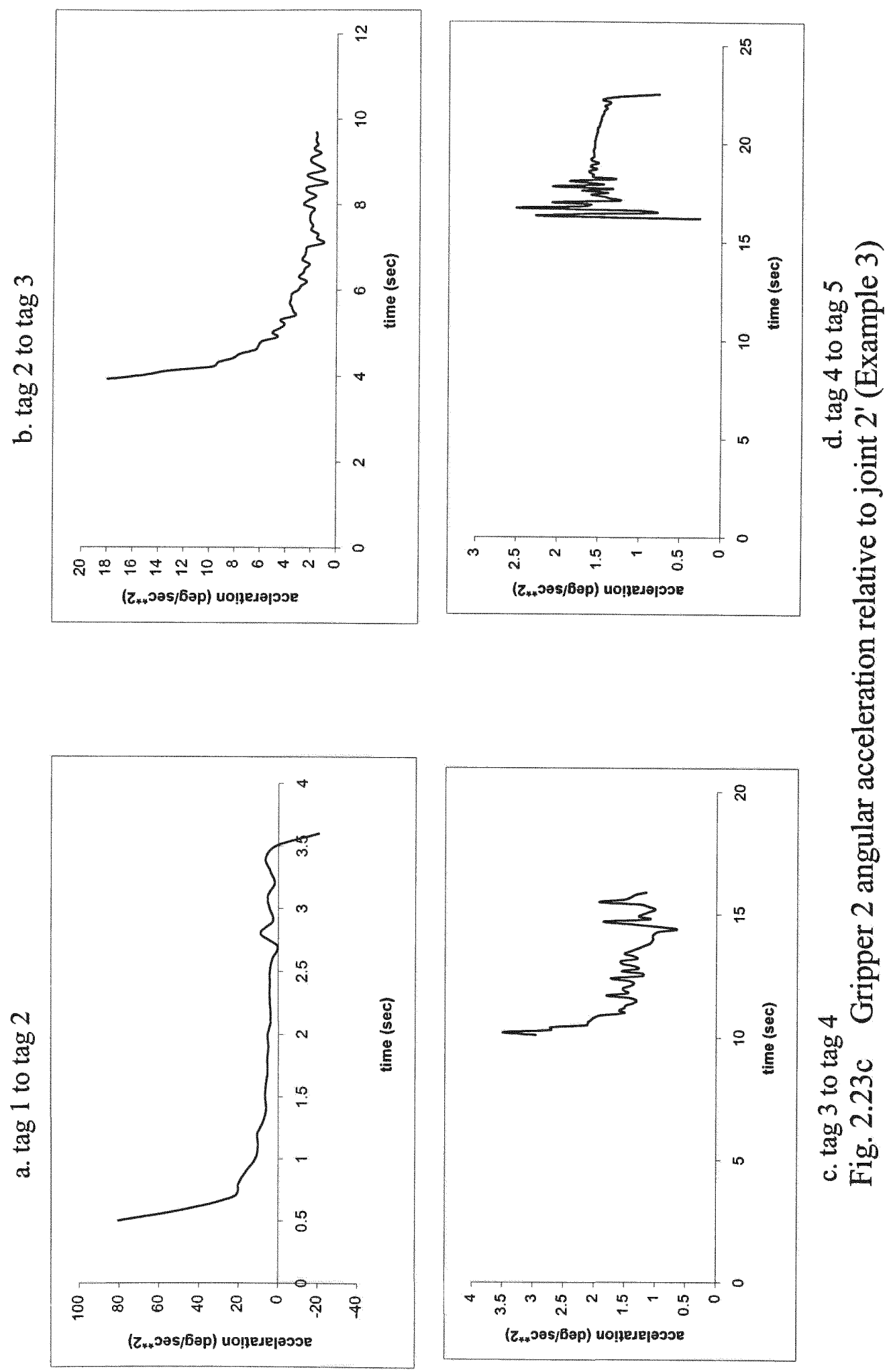

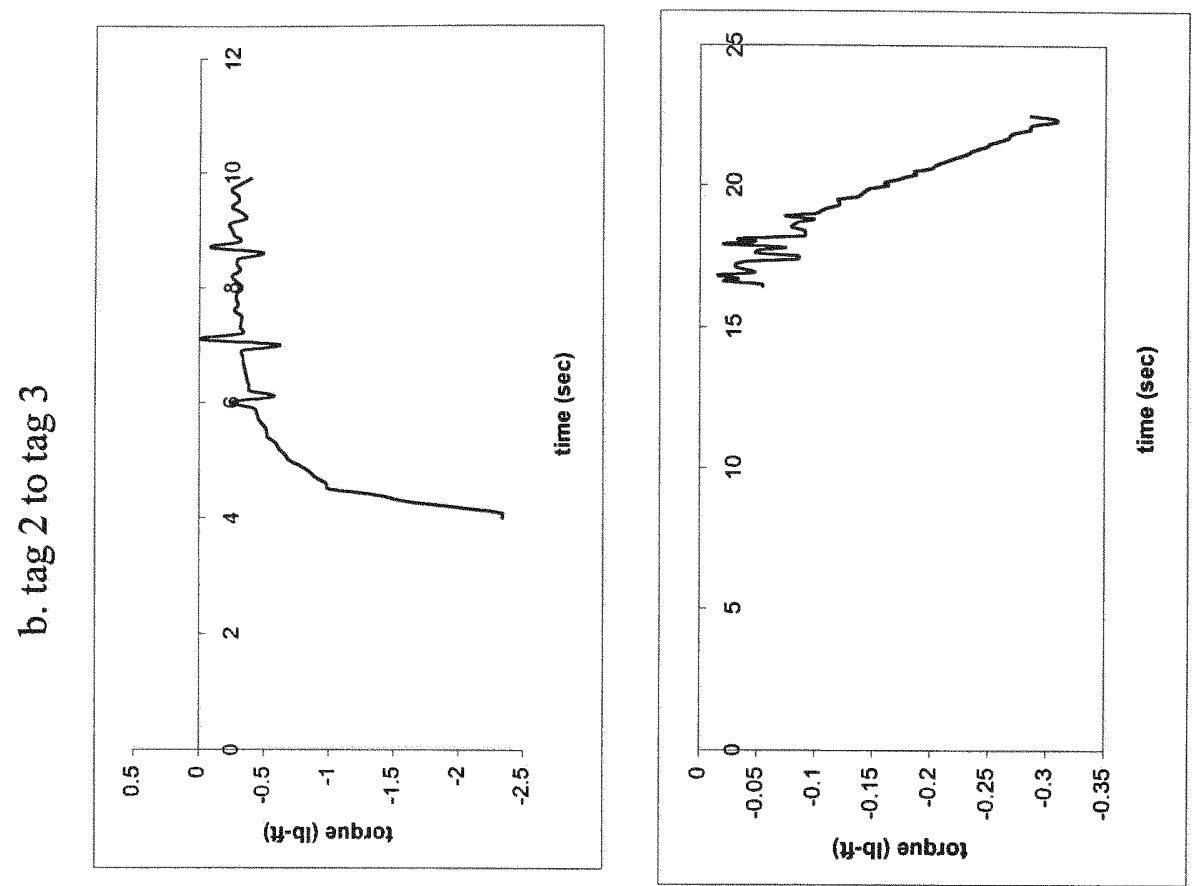

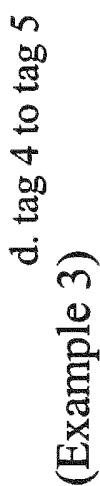
त
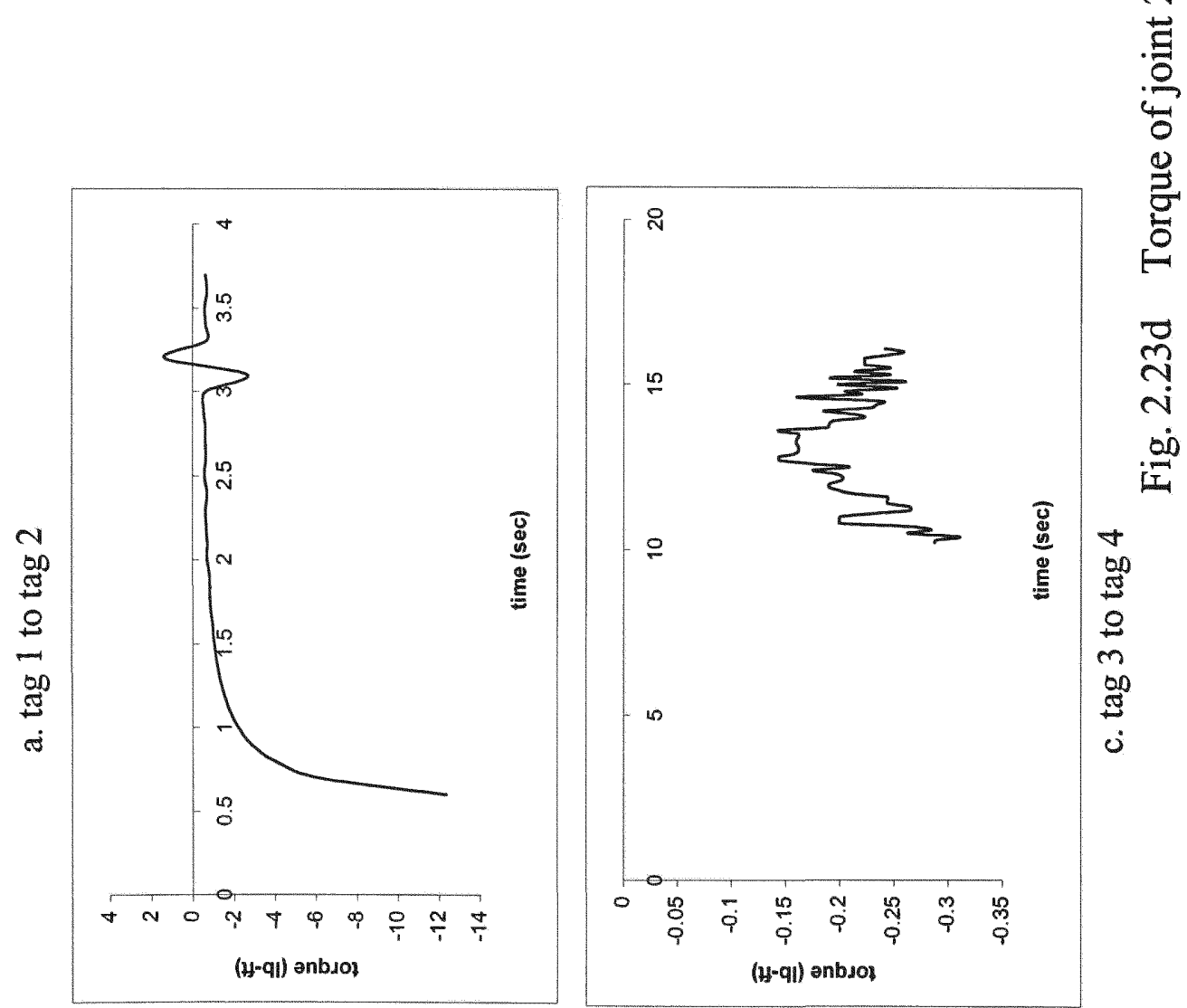

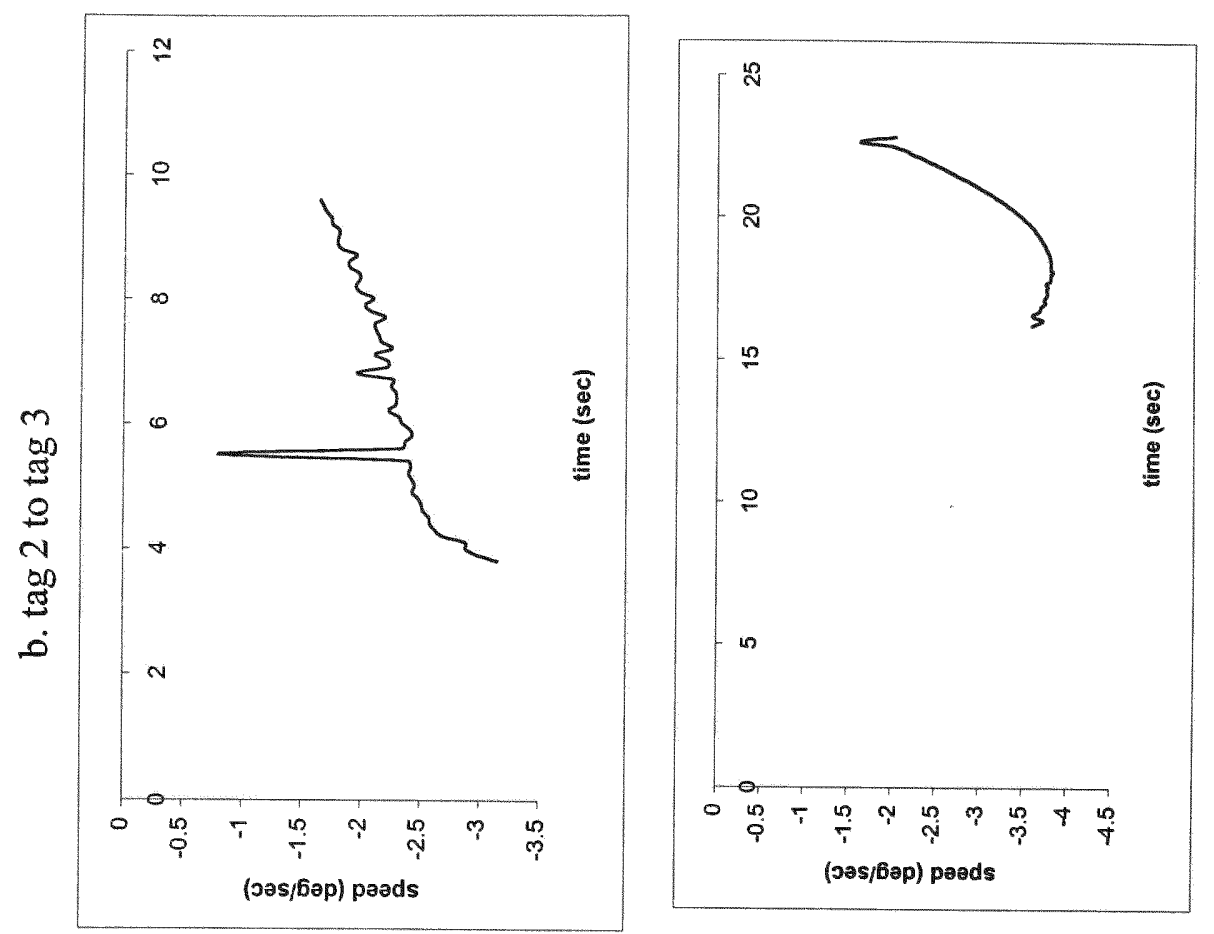

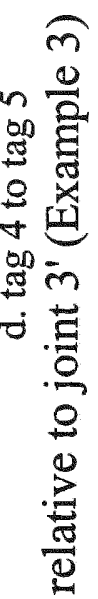
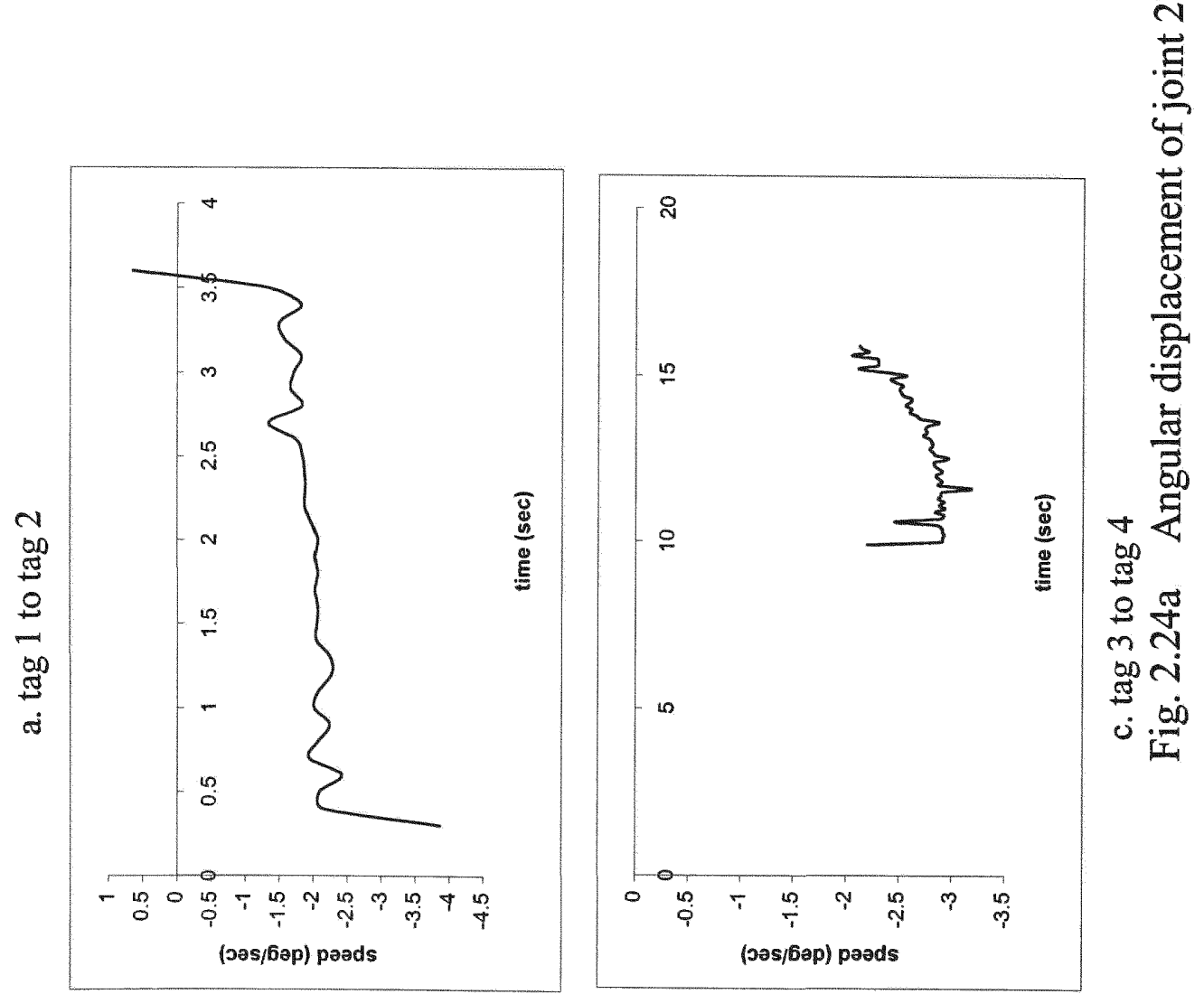

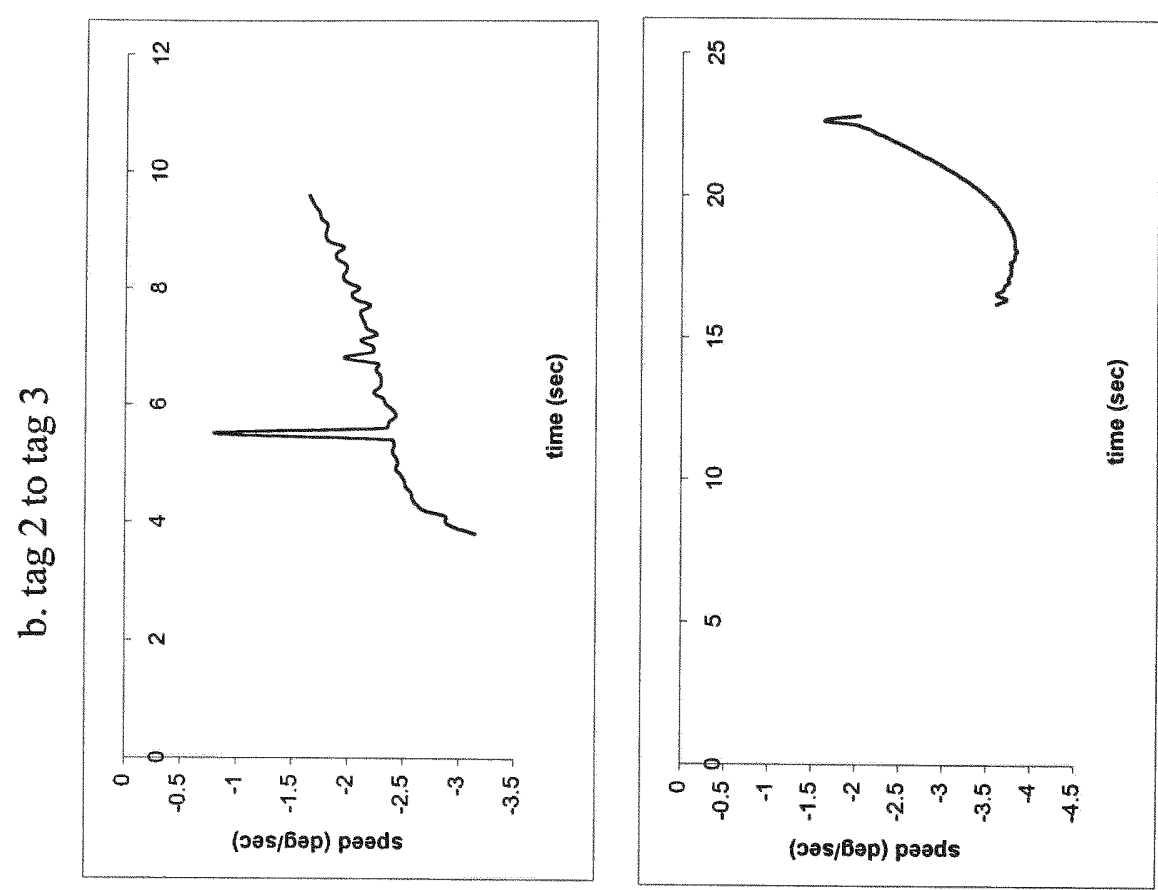

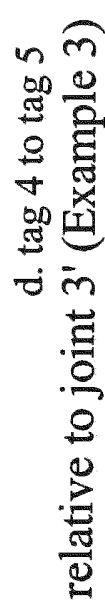
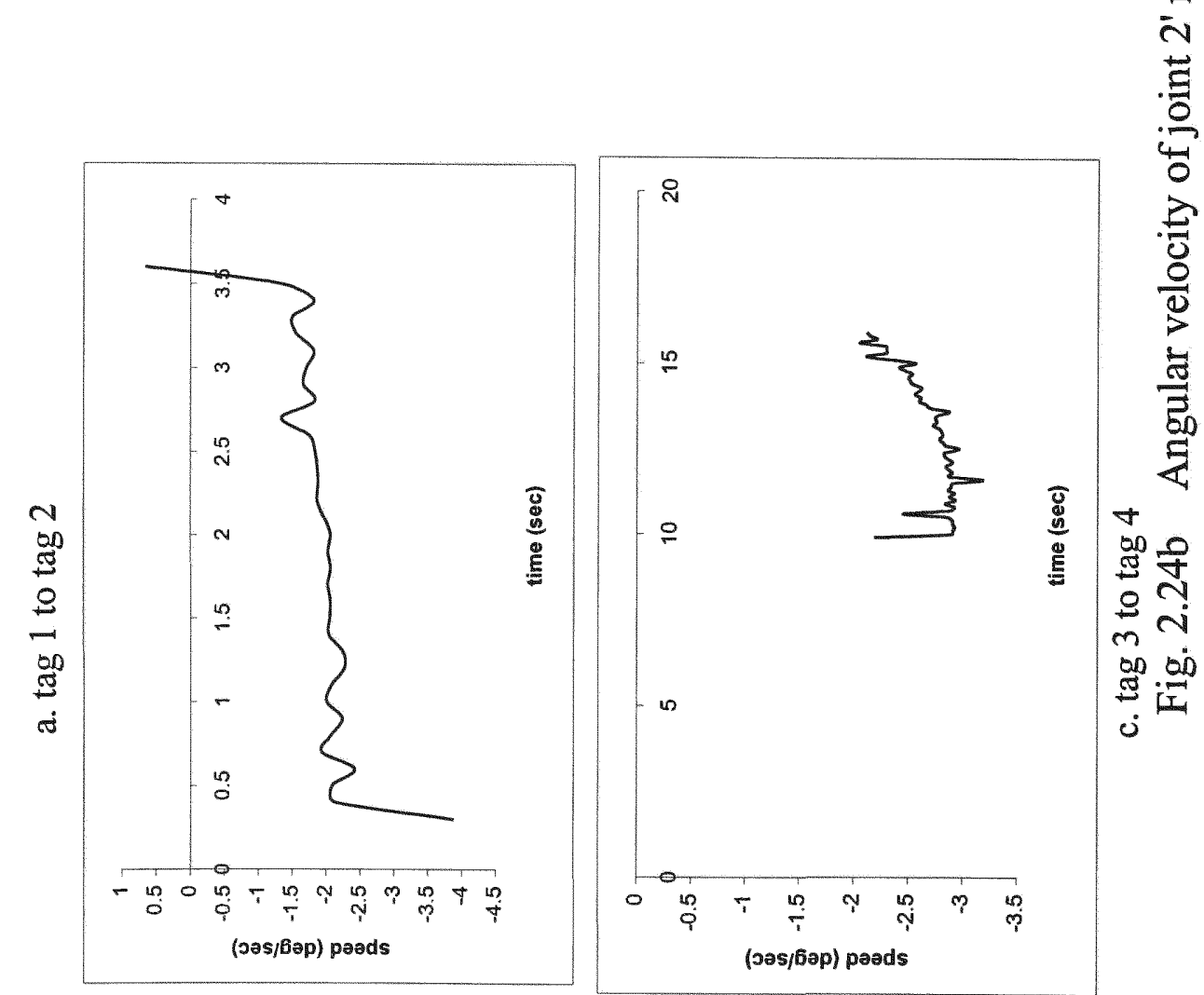

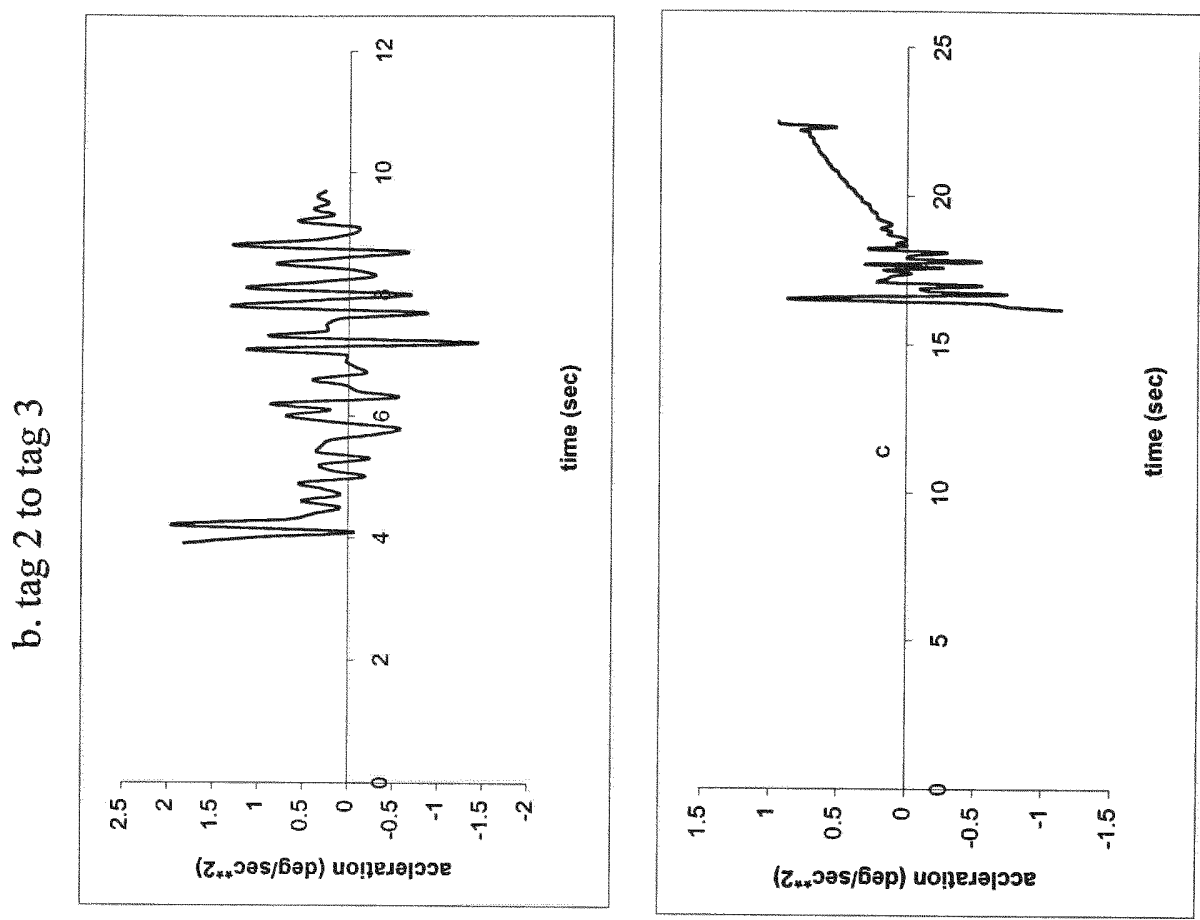

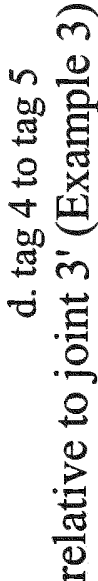
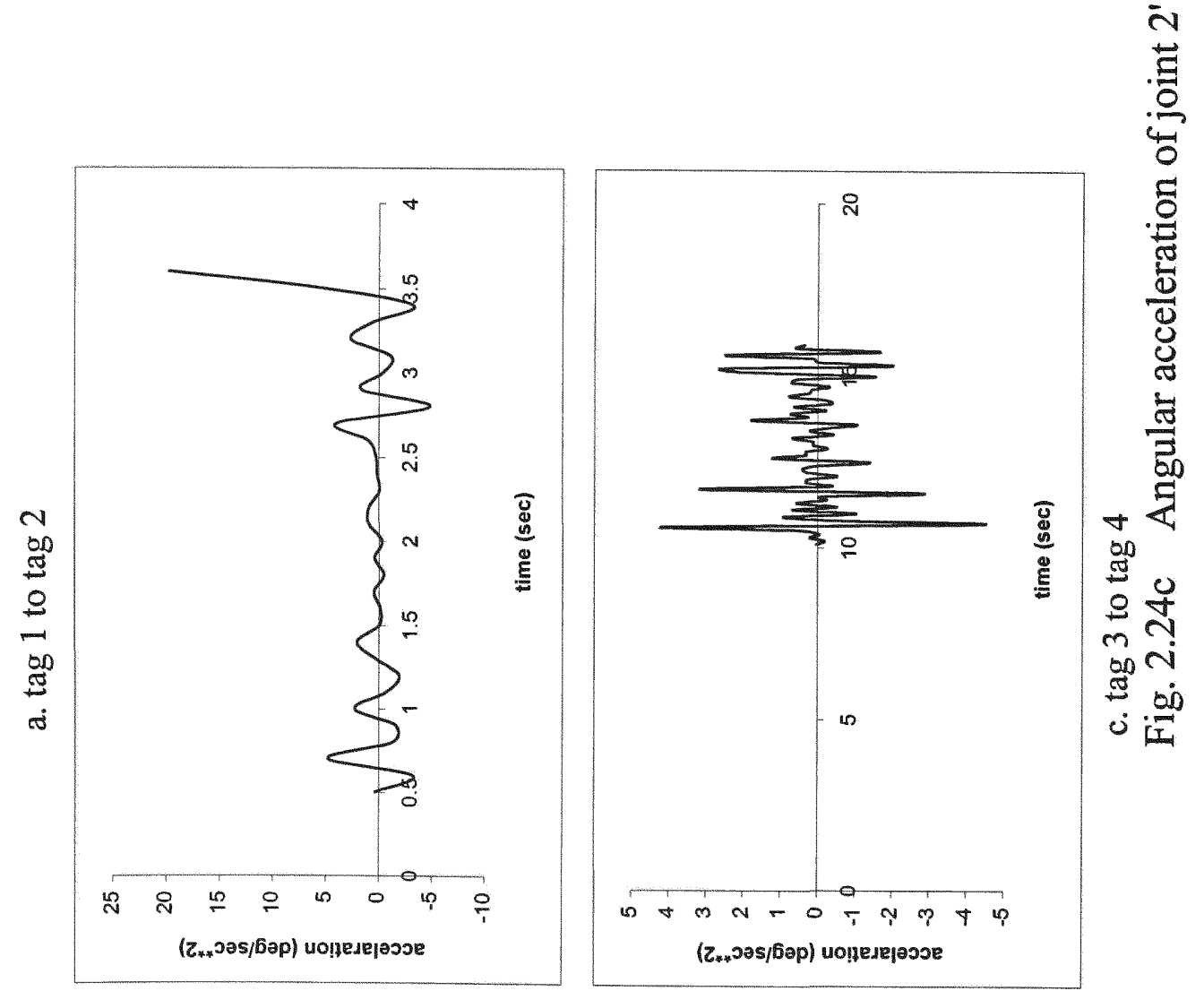

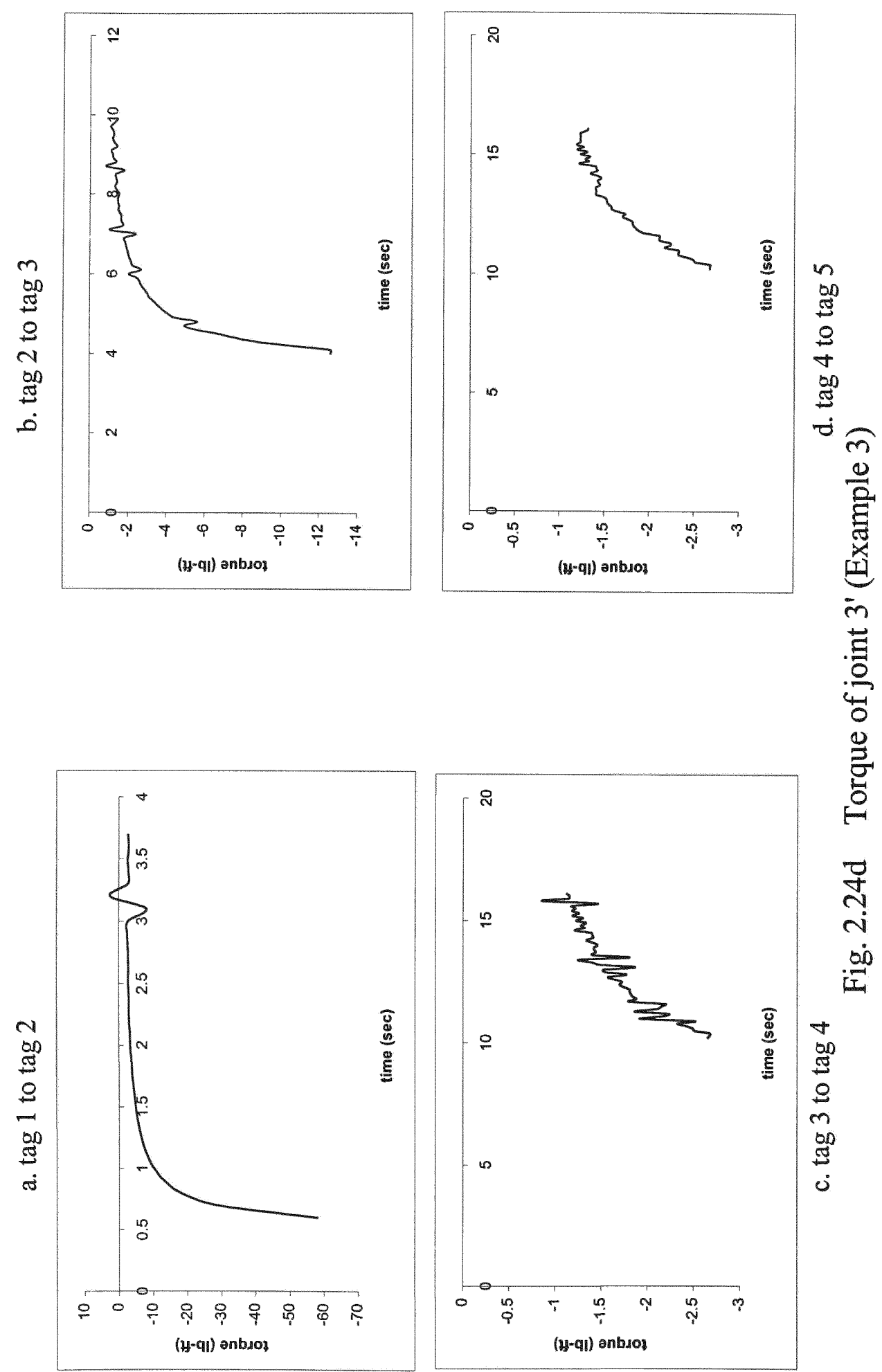

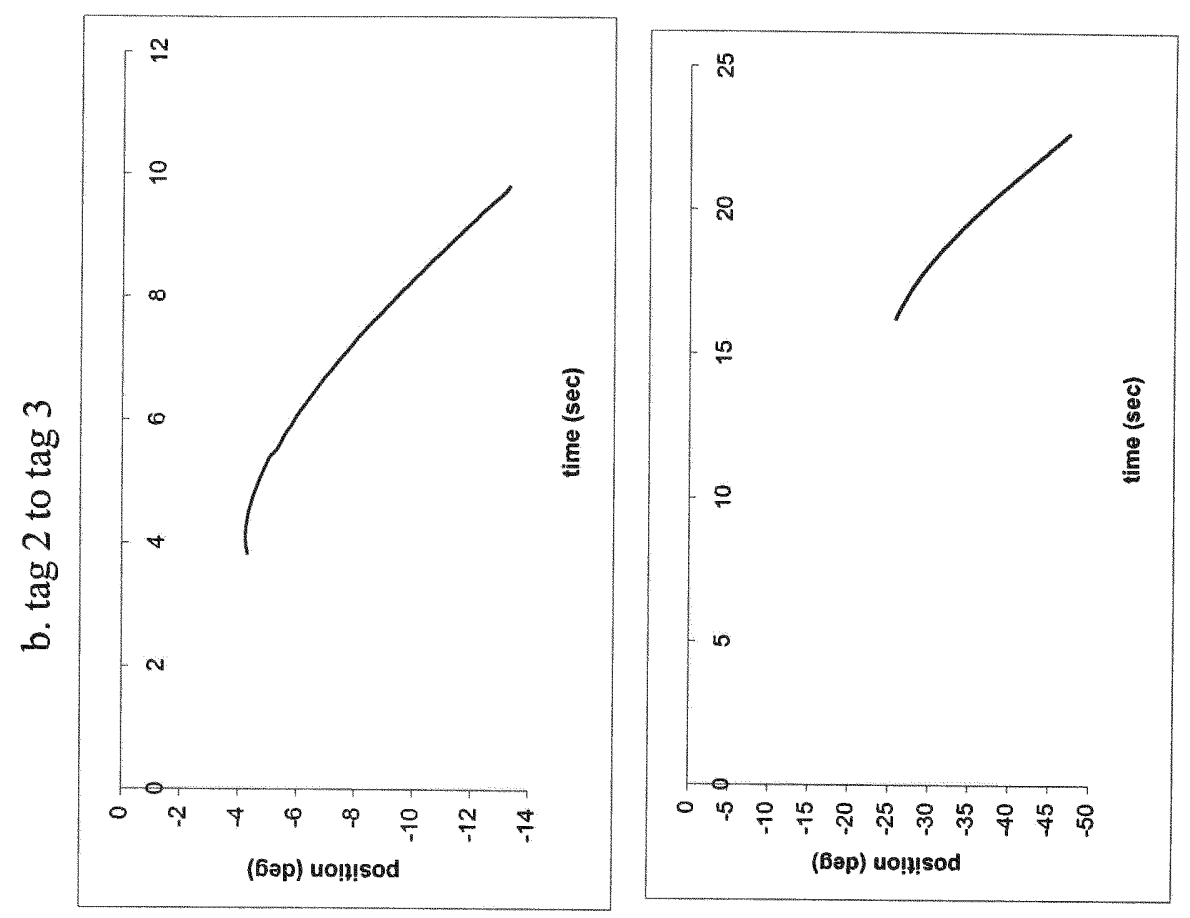

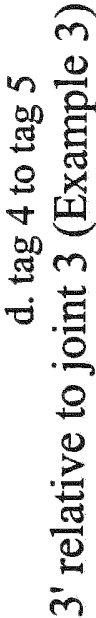

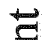

틍
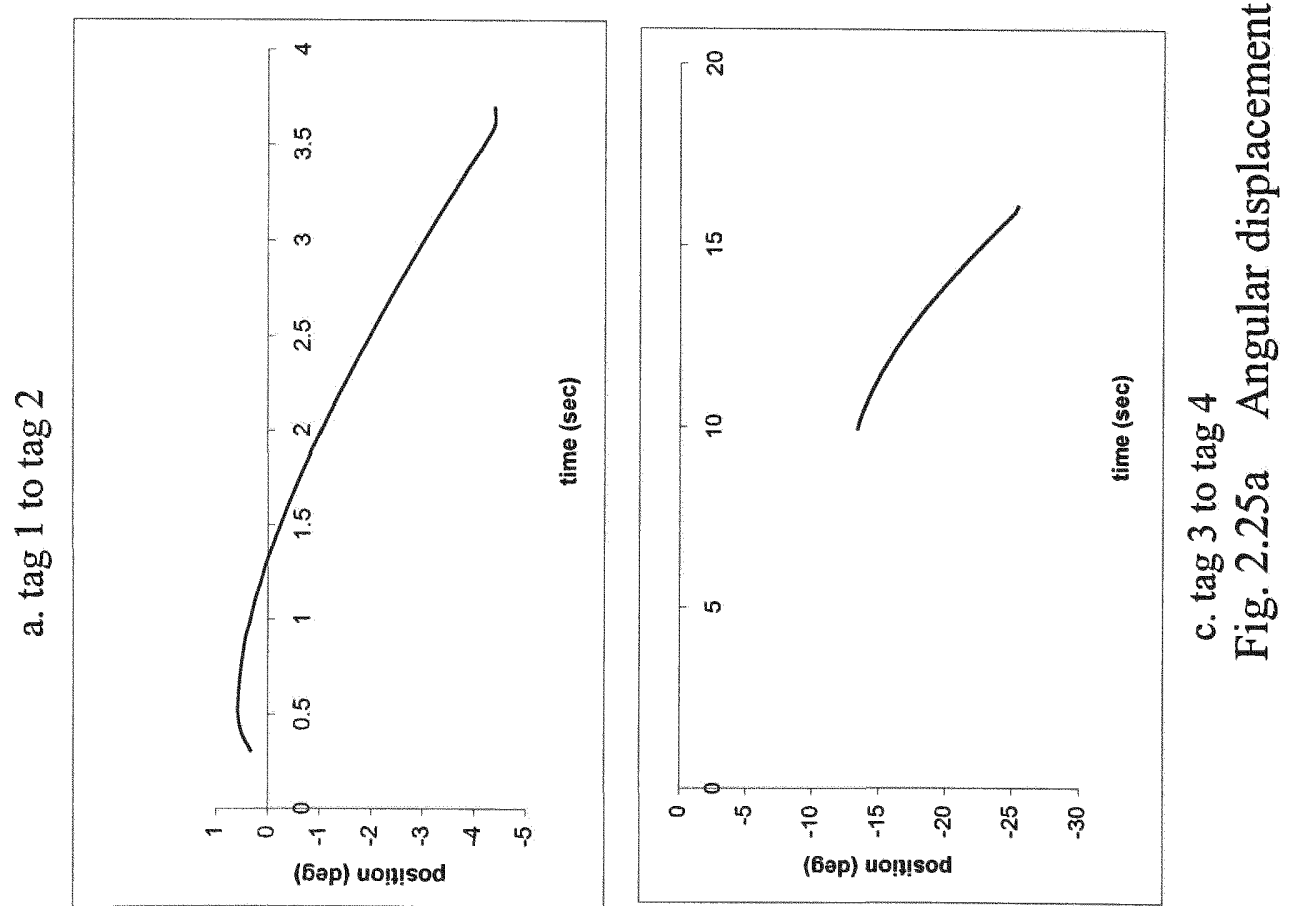

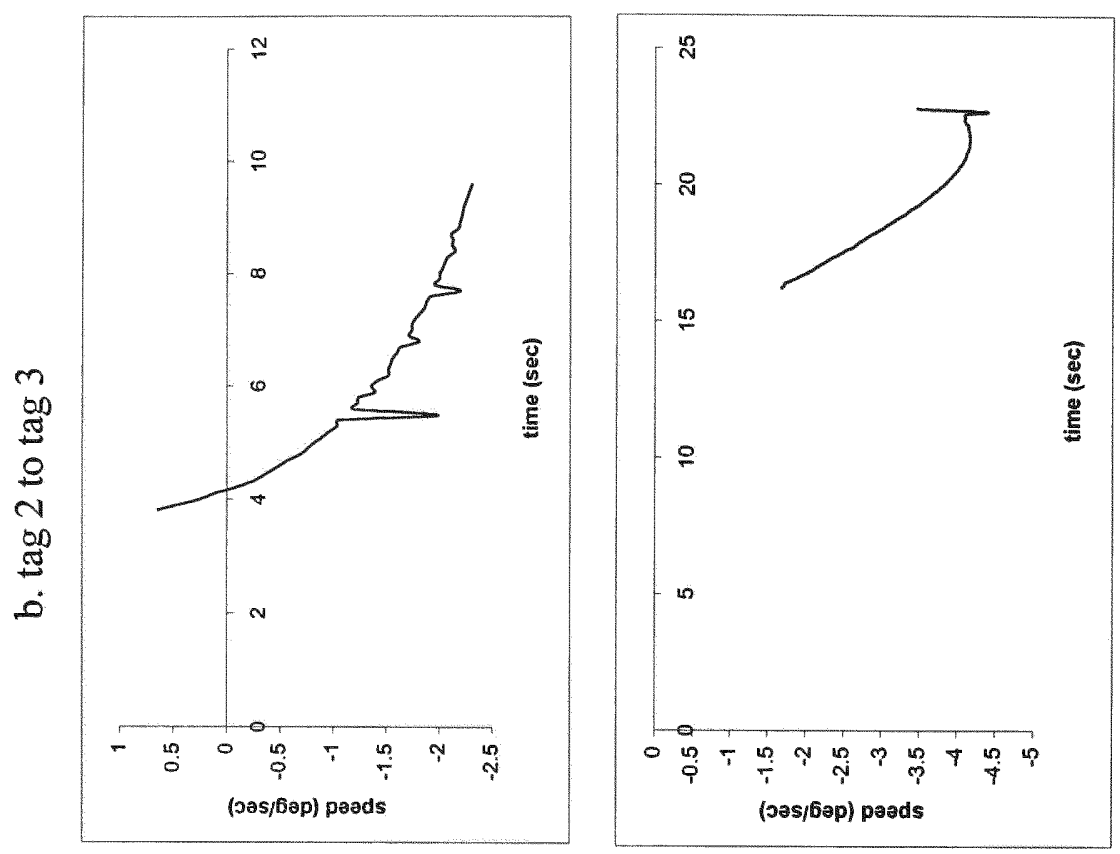

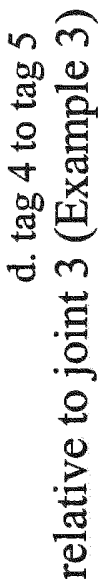
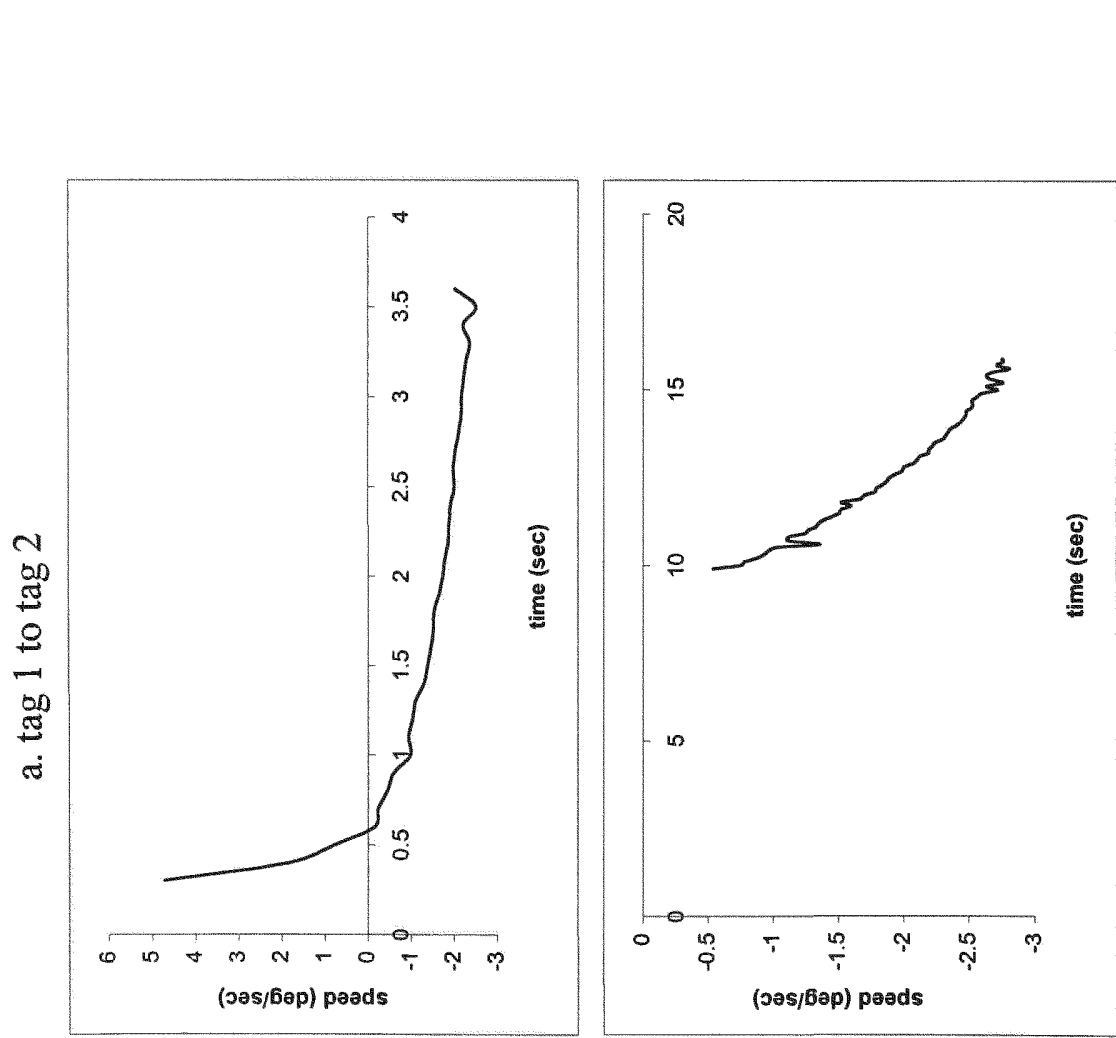

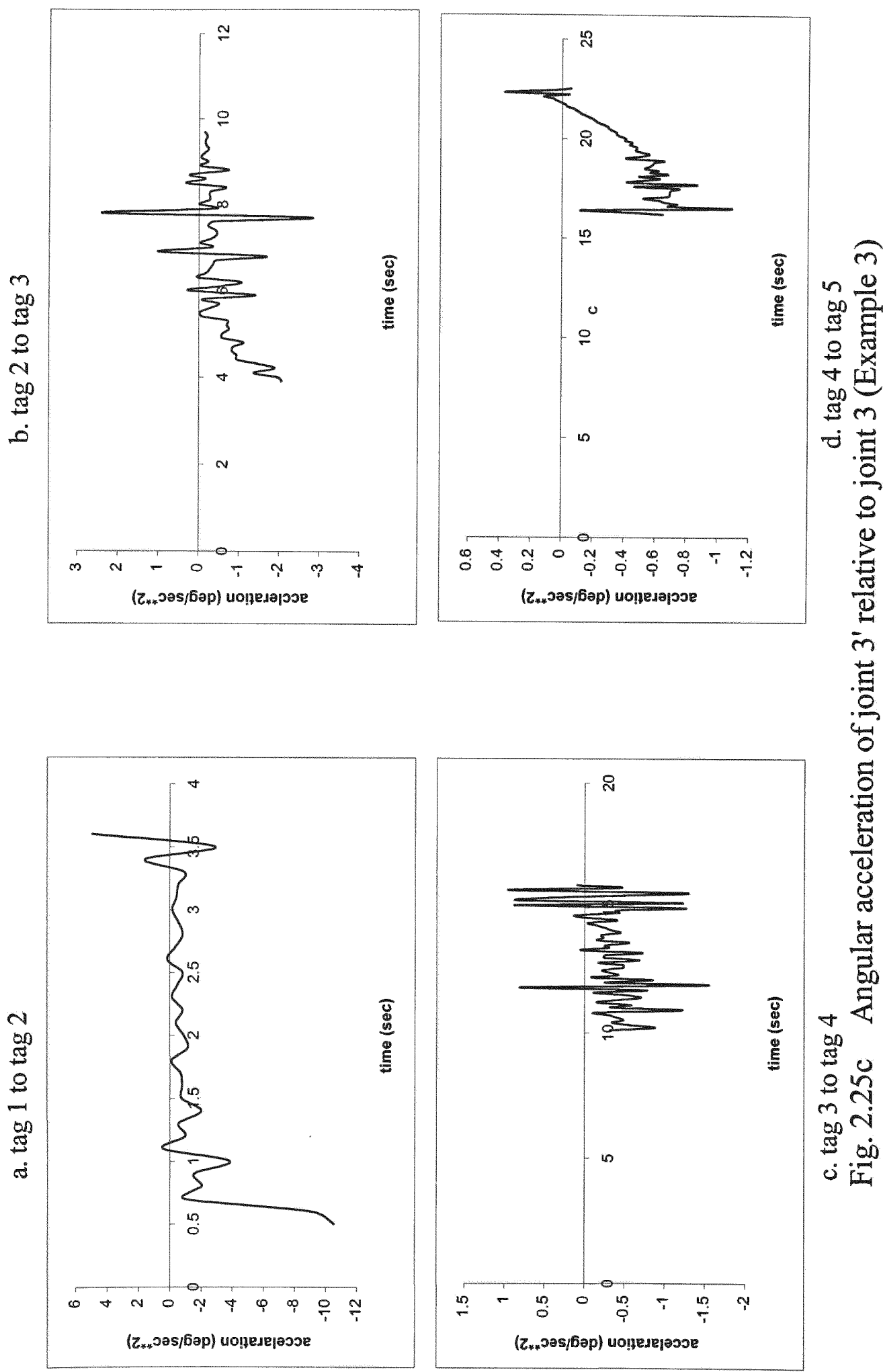

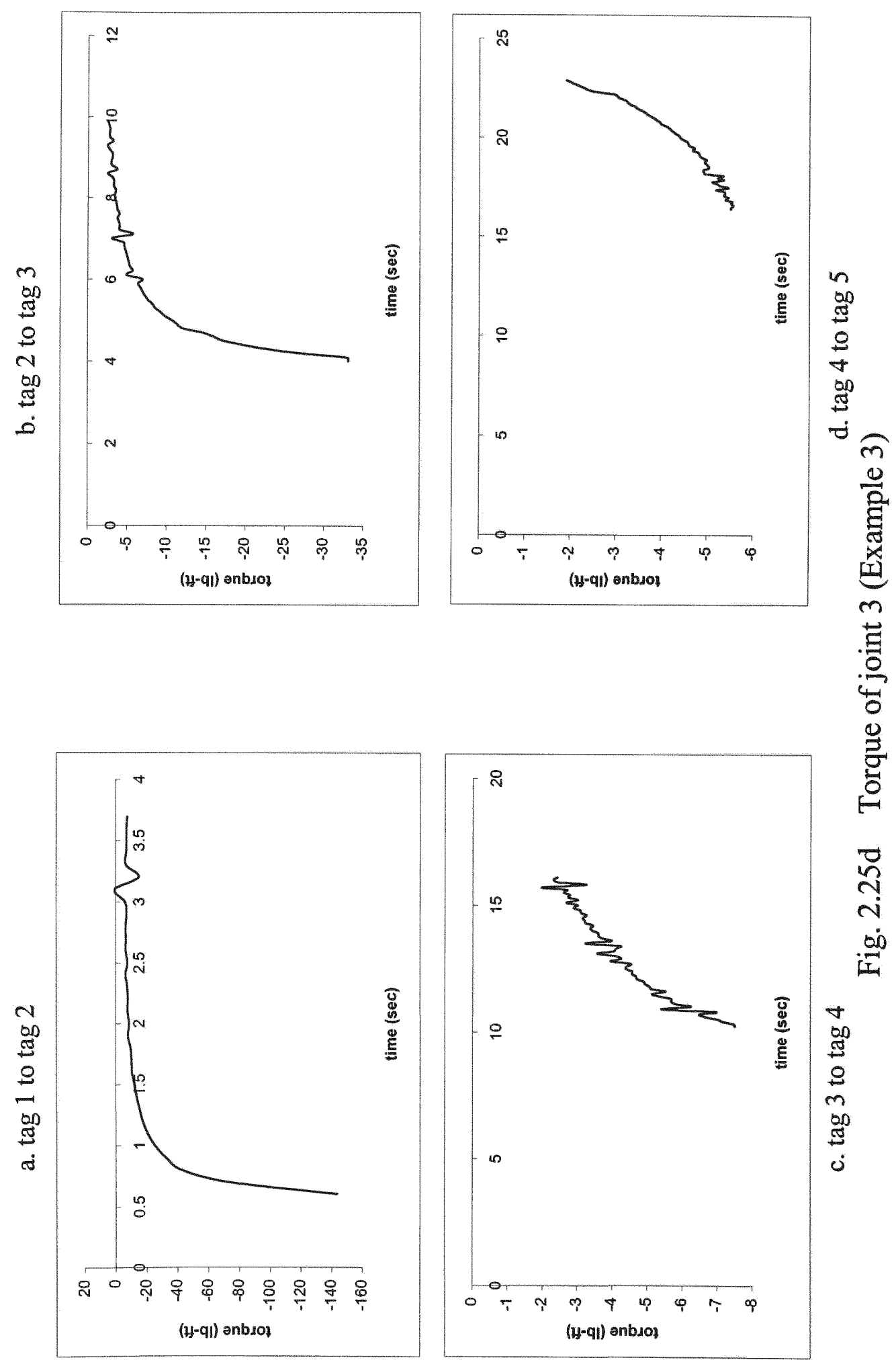

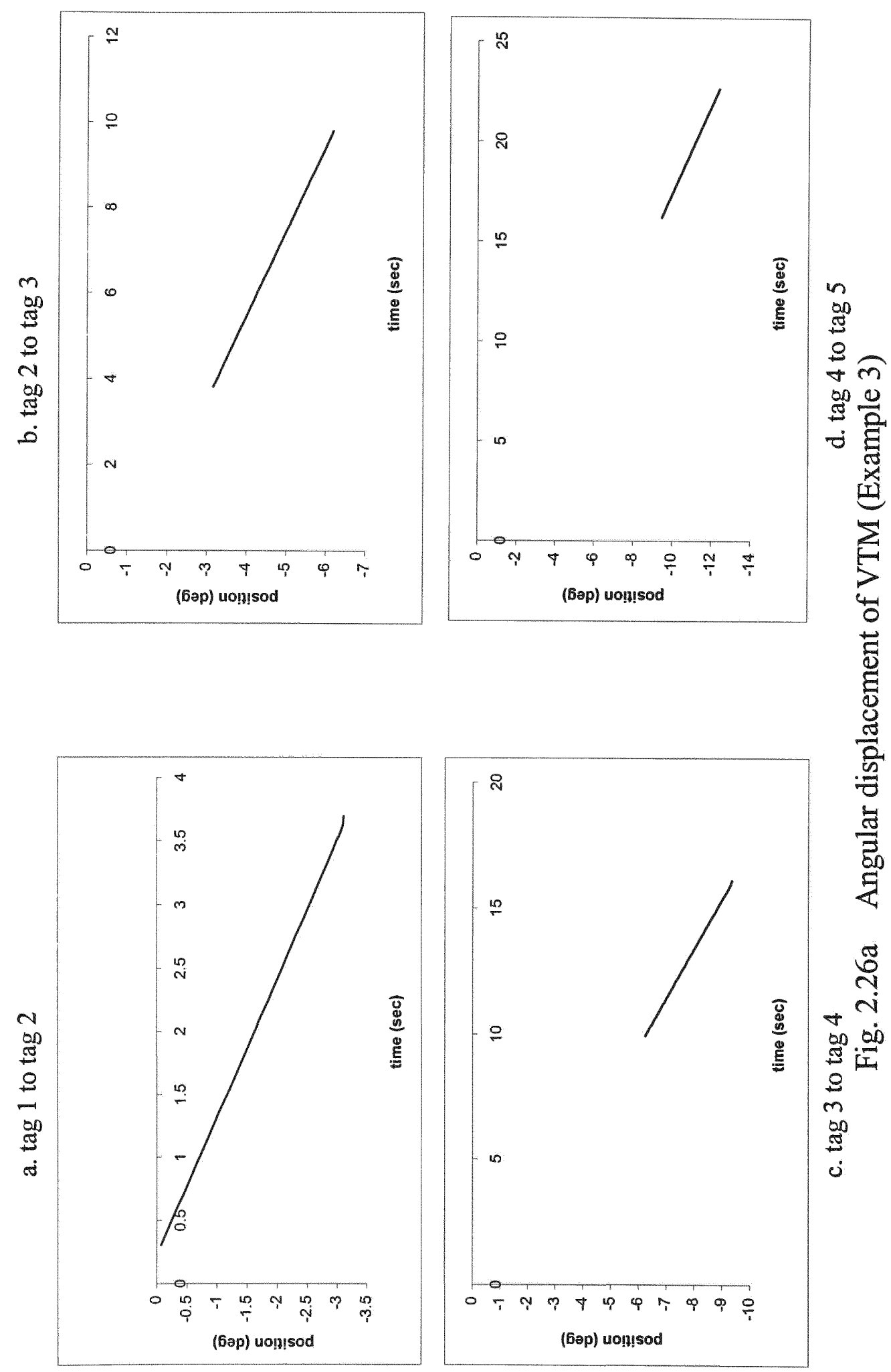

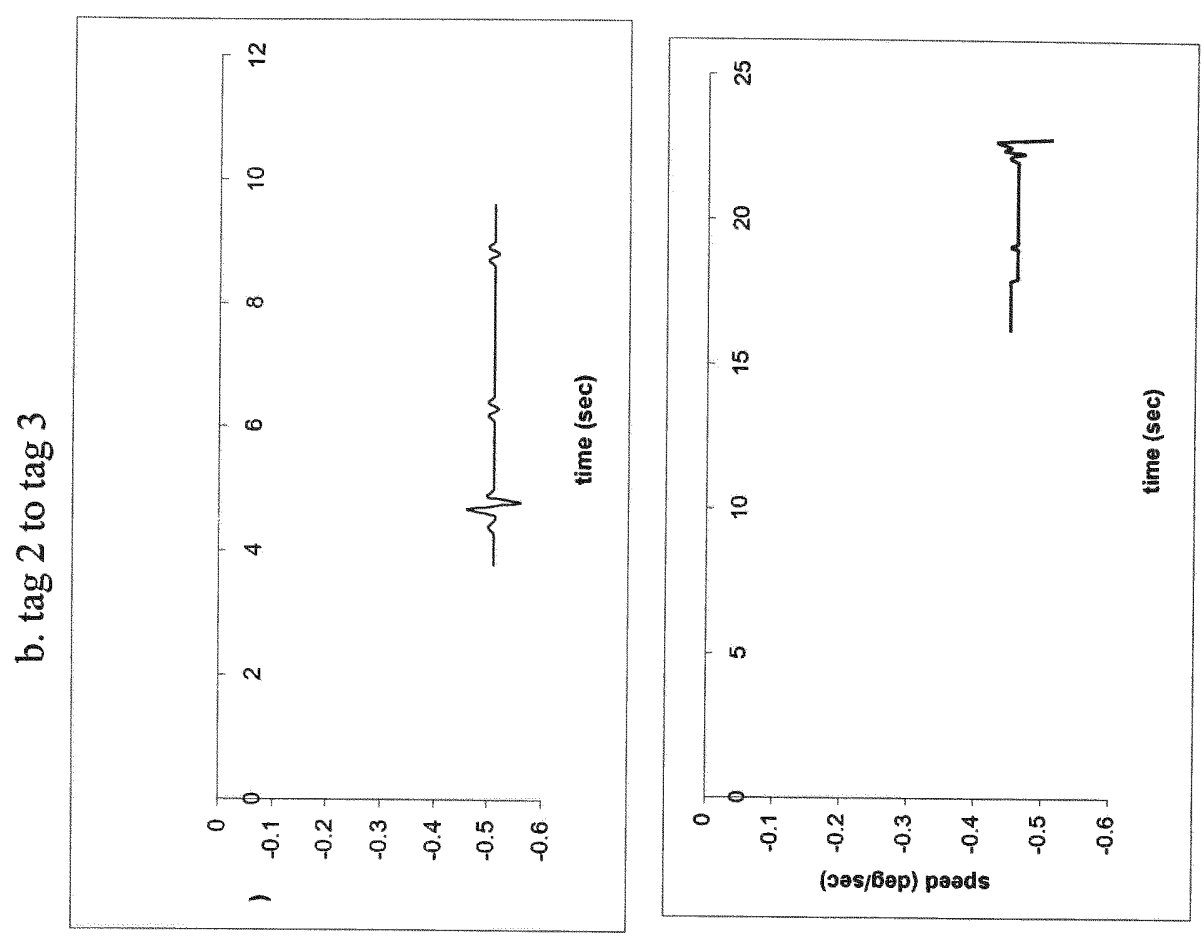

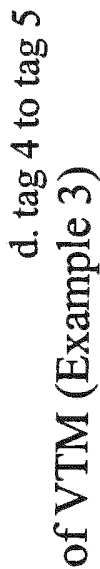

$\frac{2}{0}$
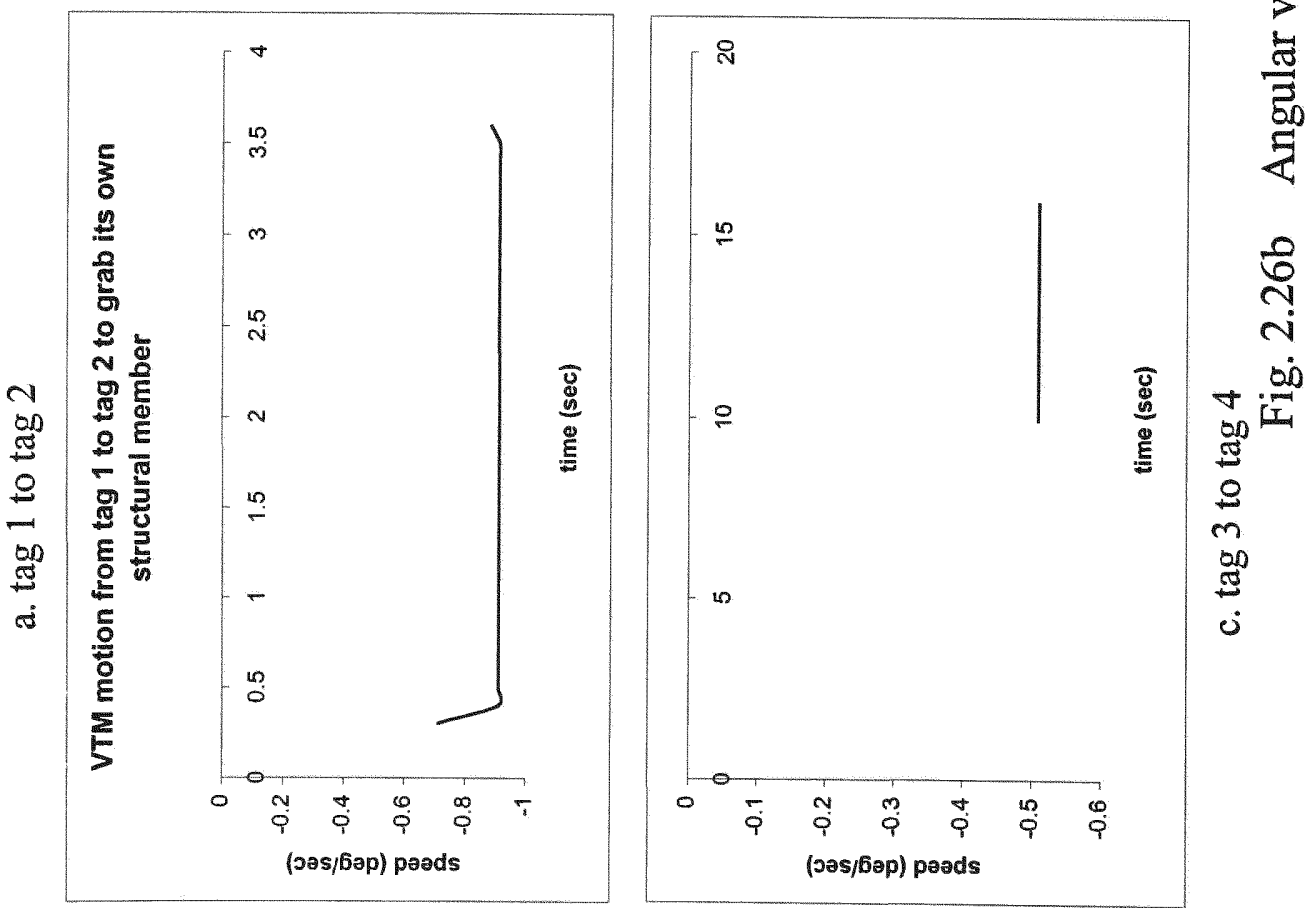

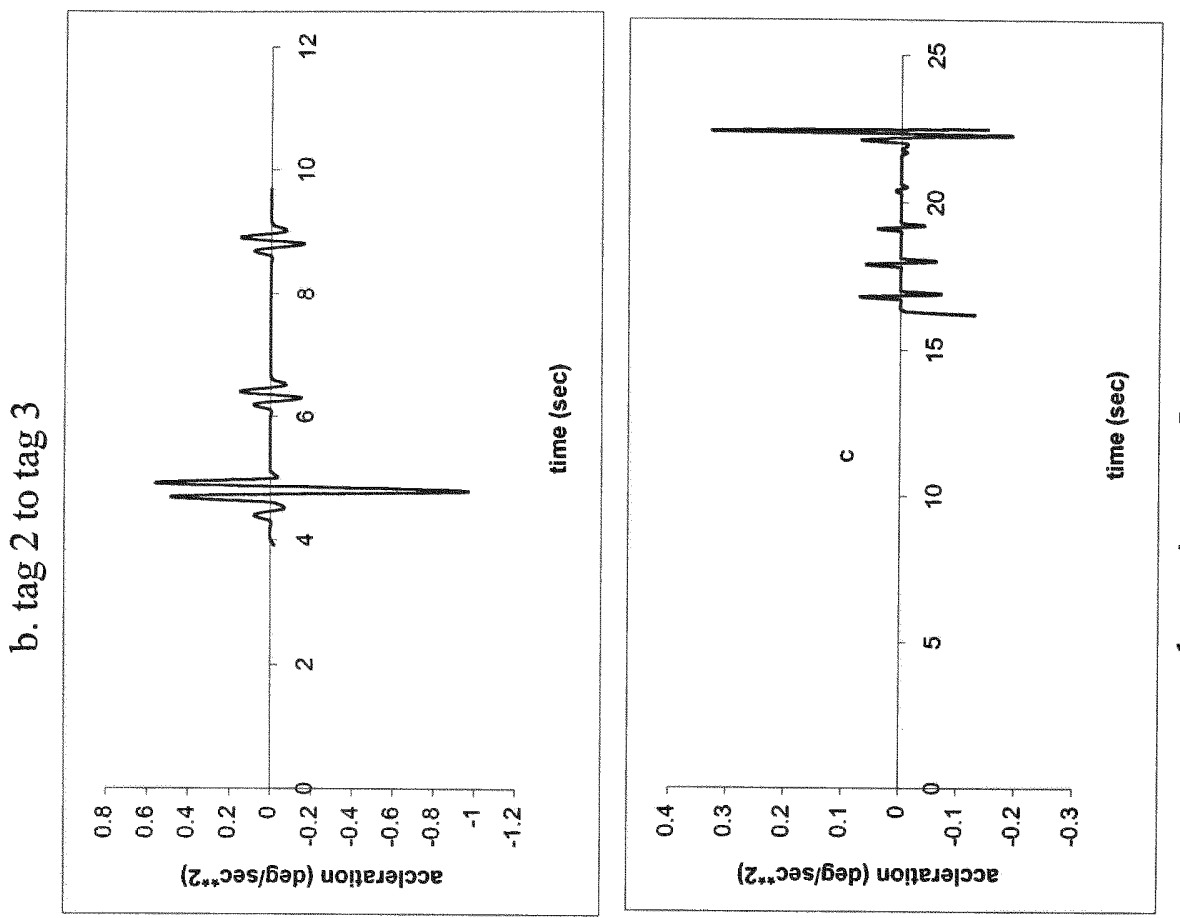

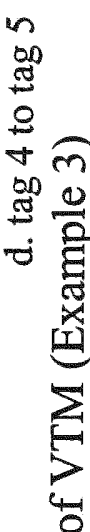
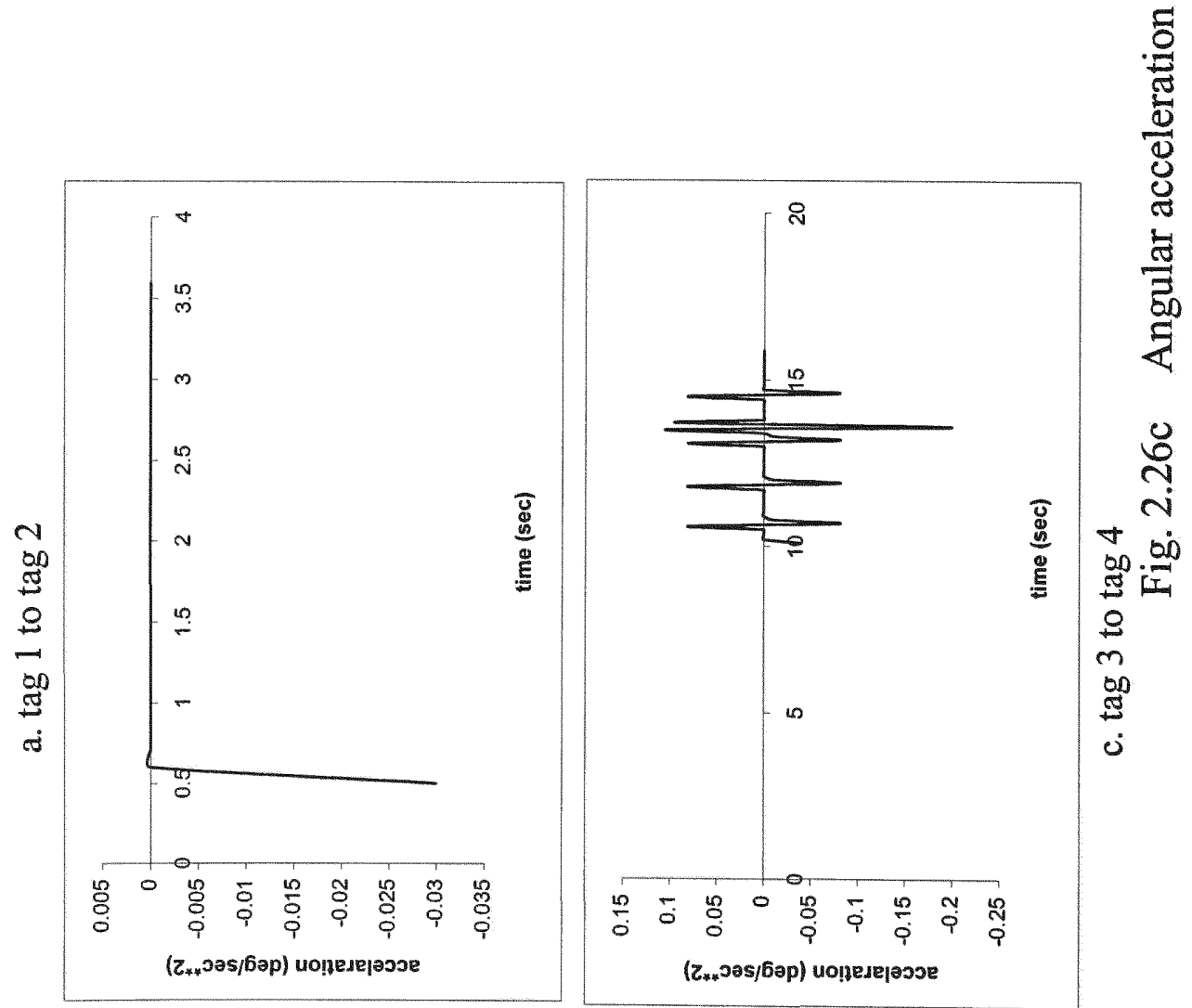

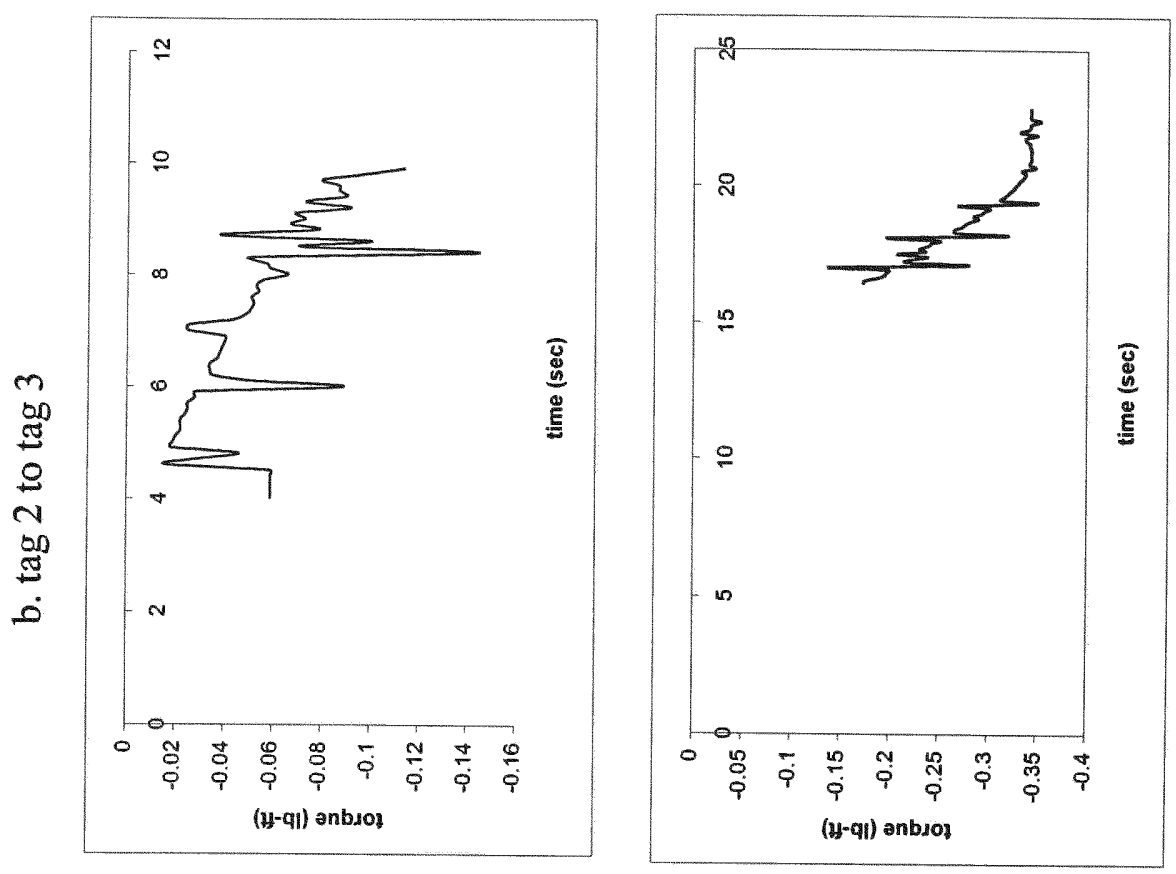

$n$
0
0
0
0
0
0
0 चं
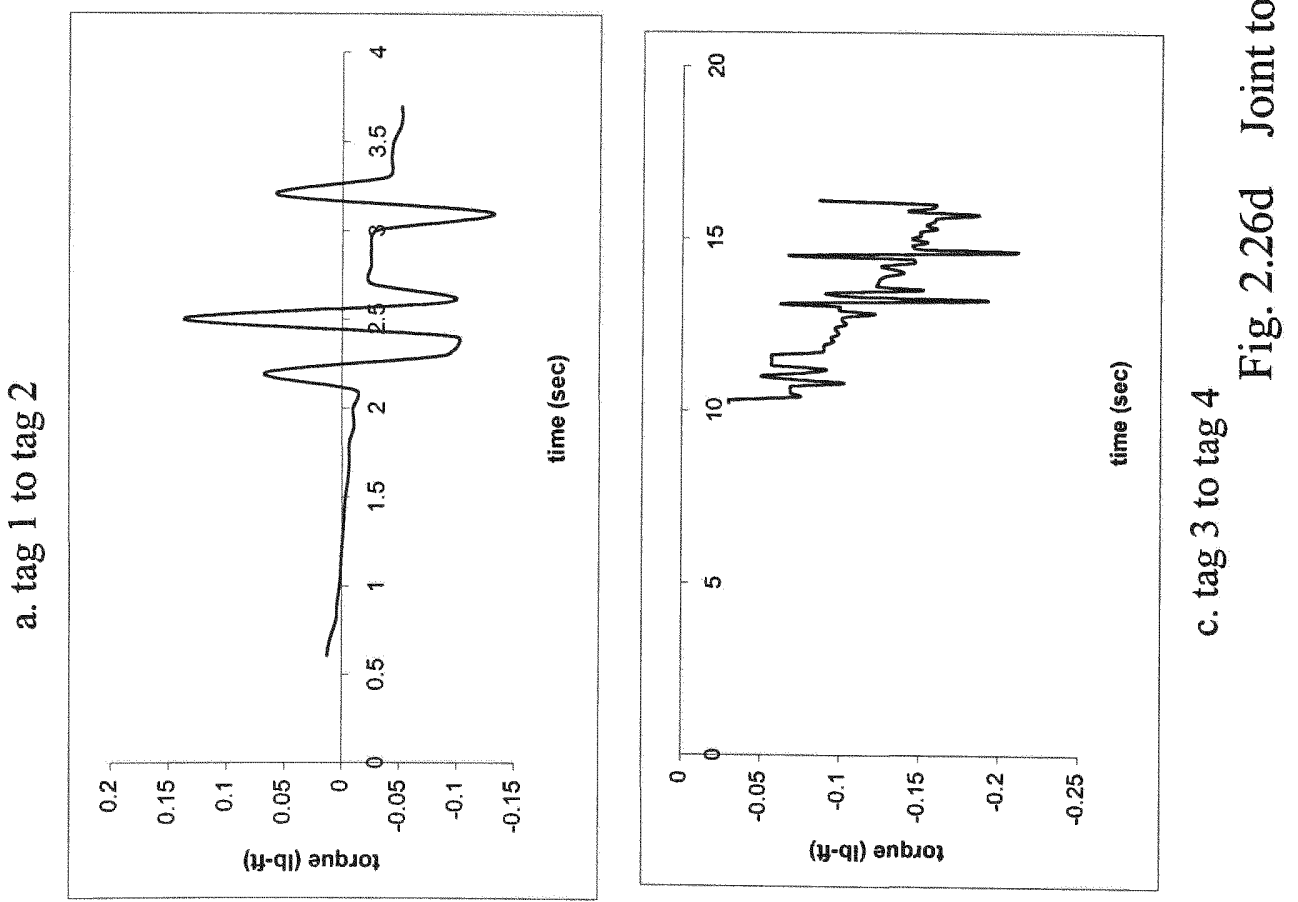

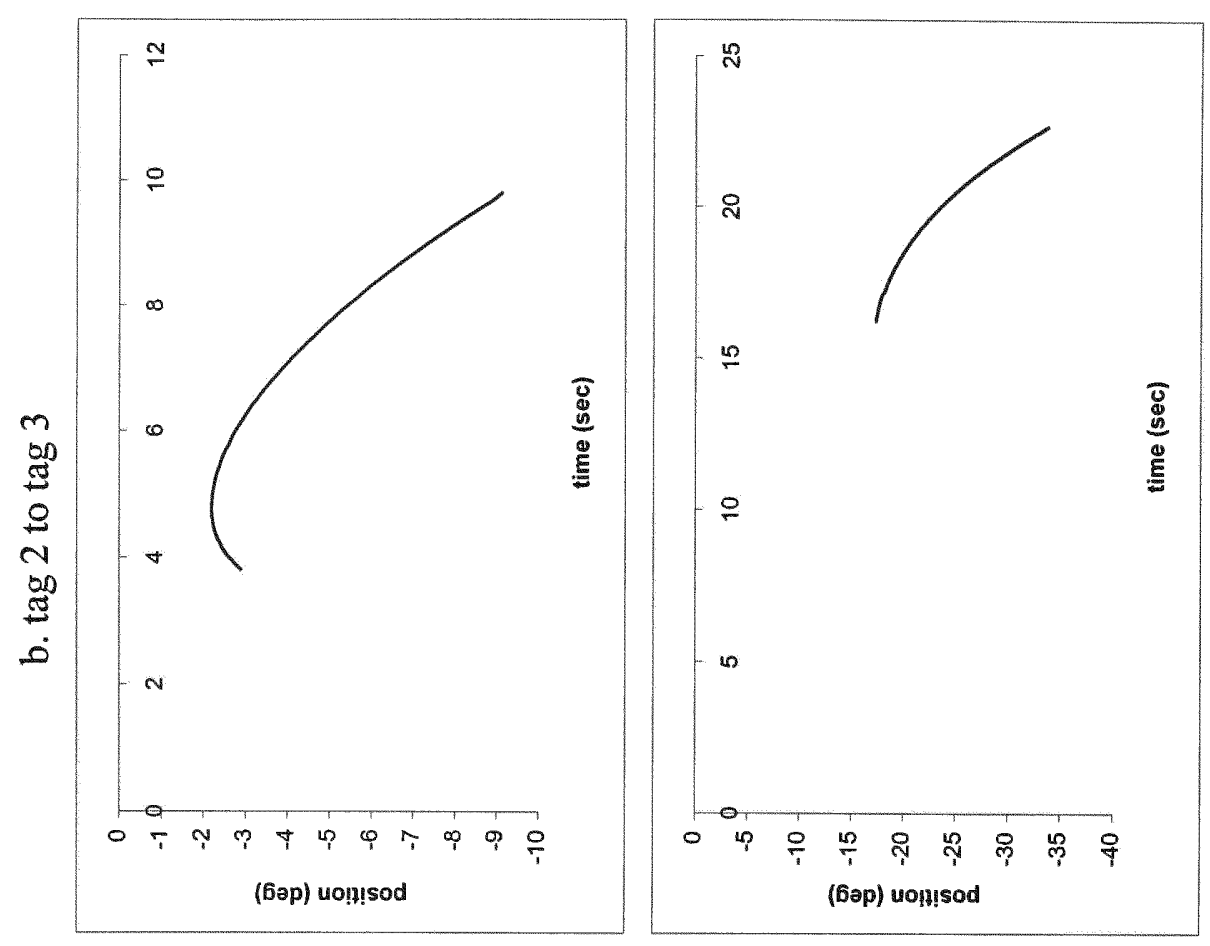

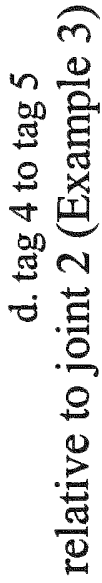
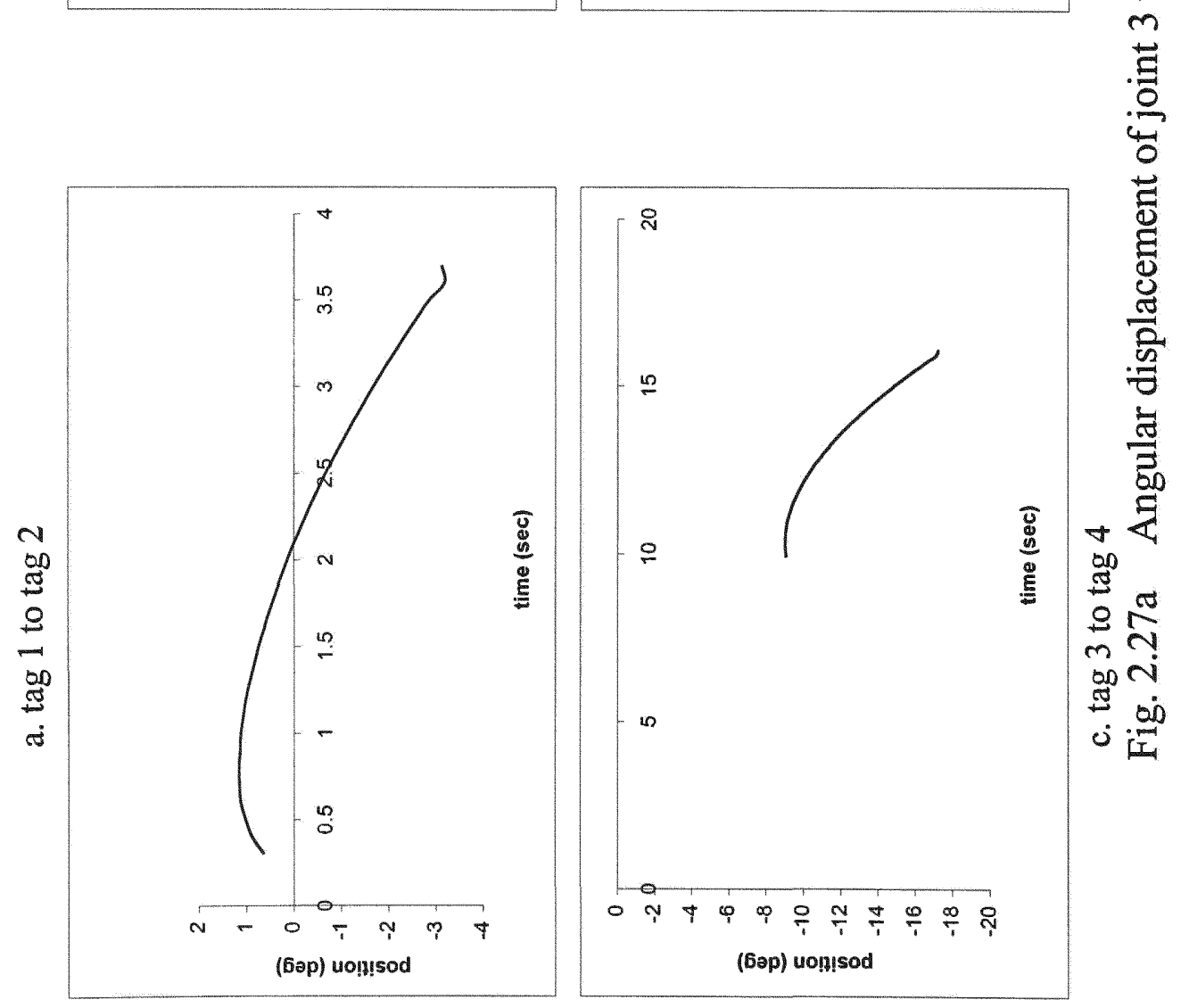

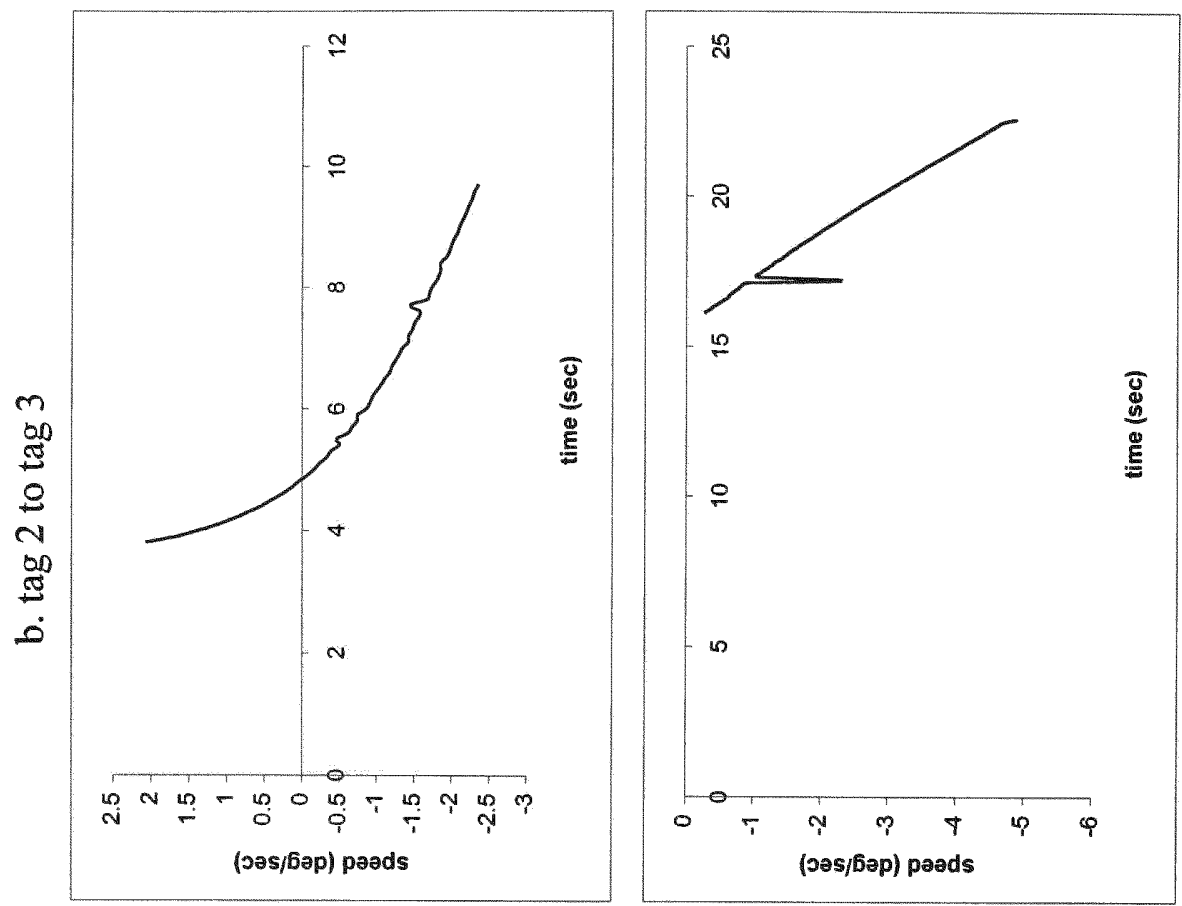

n

잉을

요

on

四

ชं

틍

용

$\frac{2}{2}$

m

Б

4
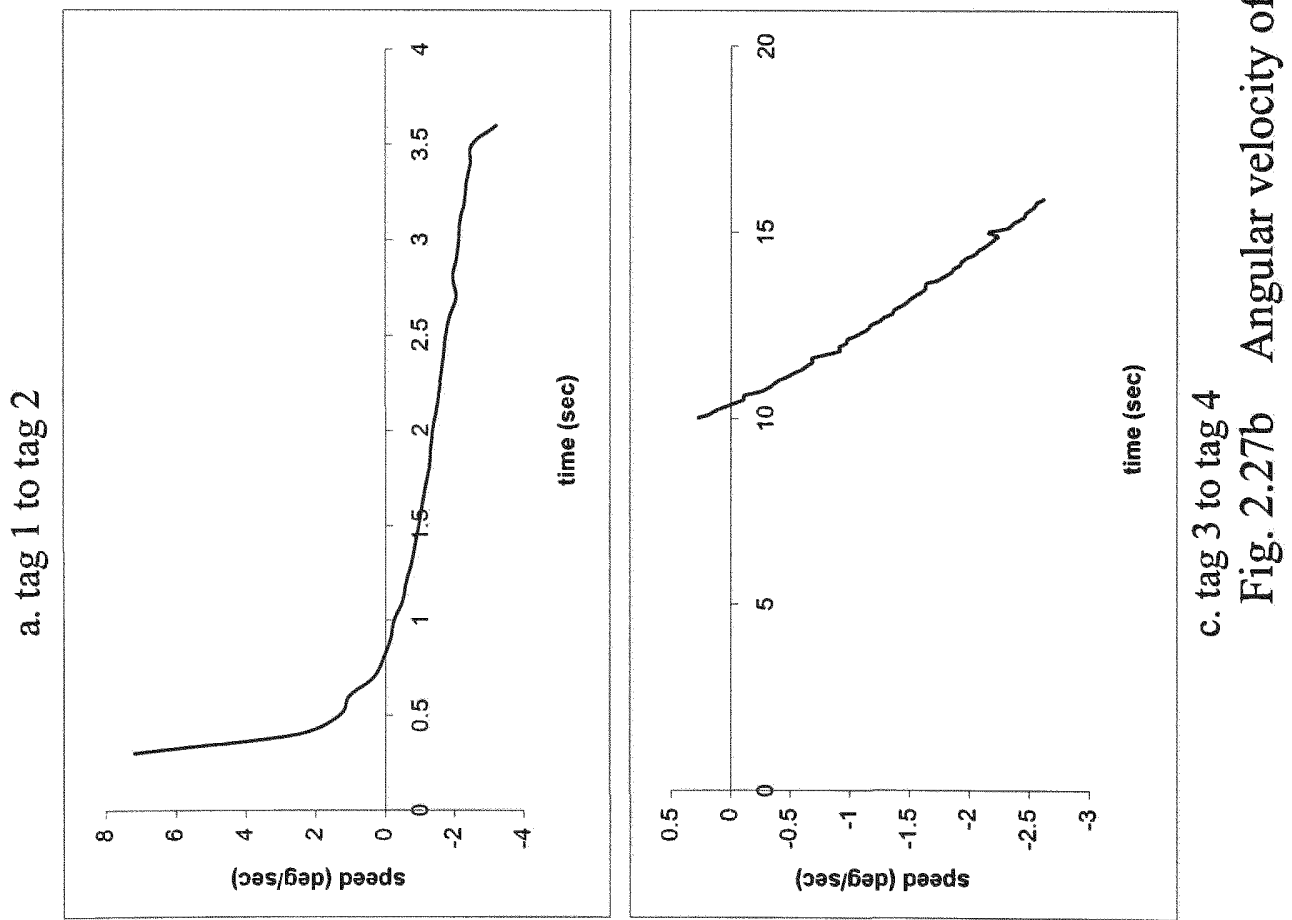

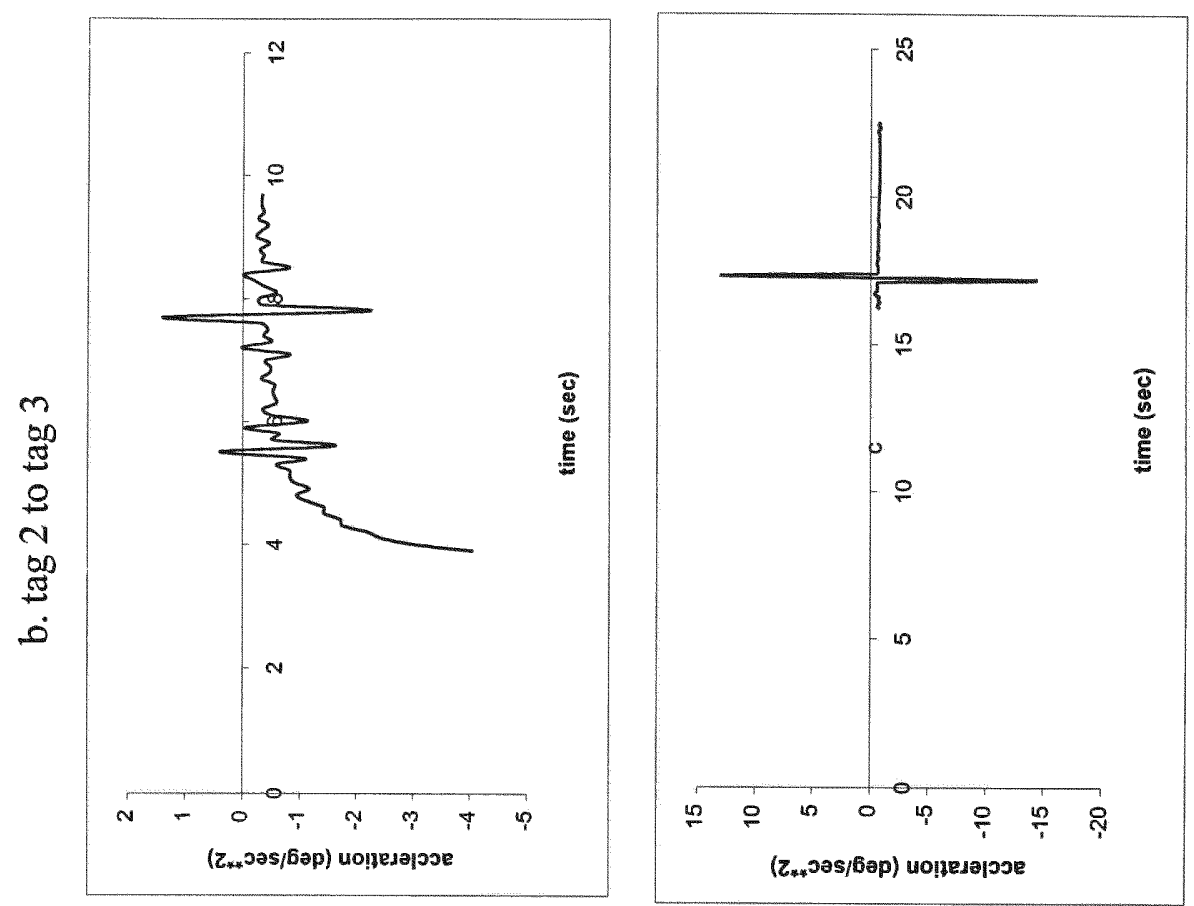

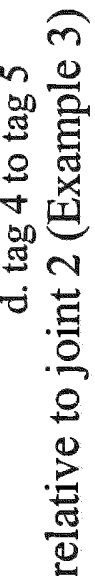

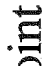
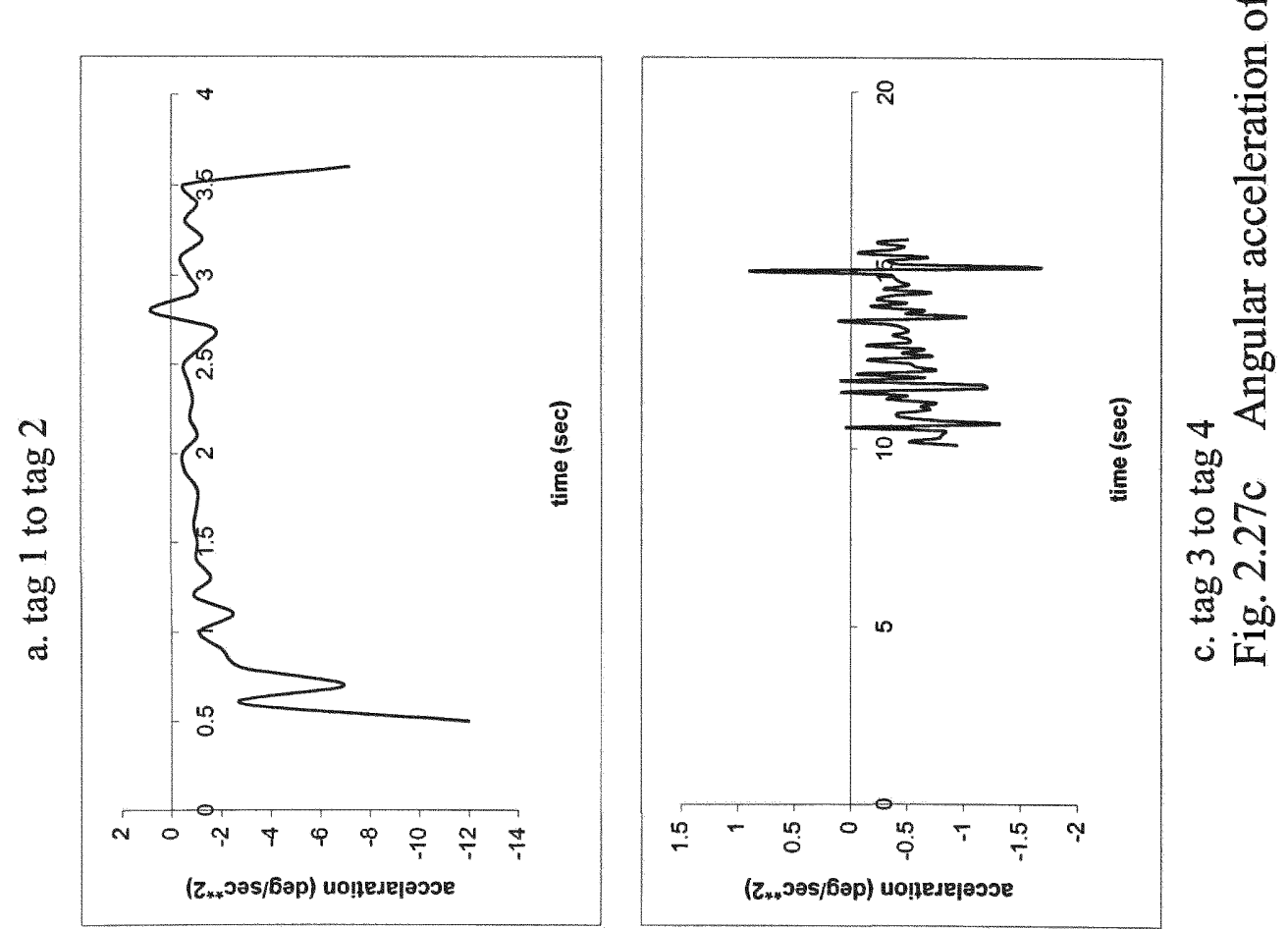

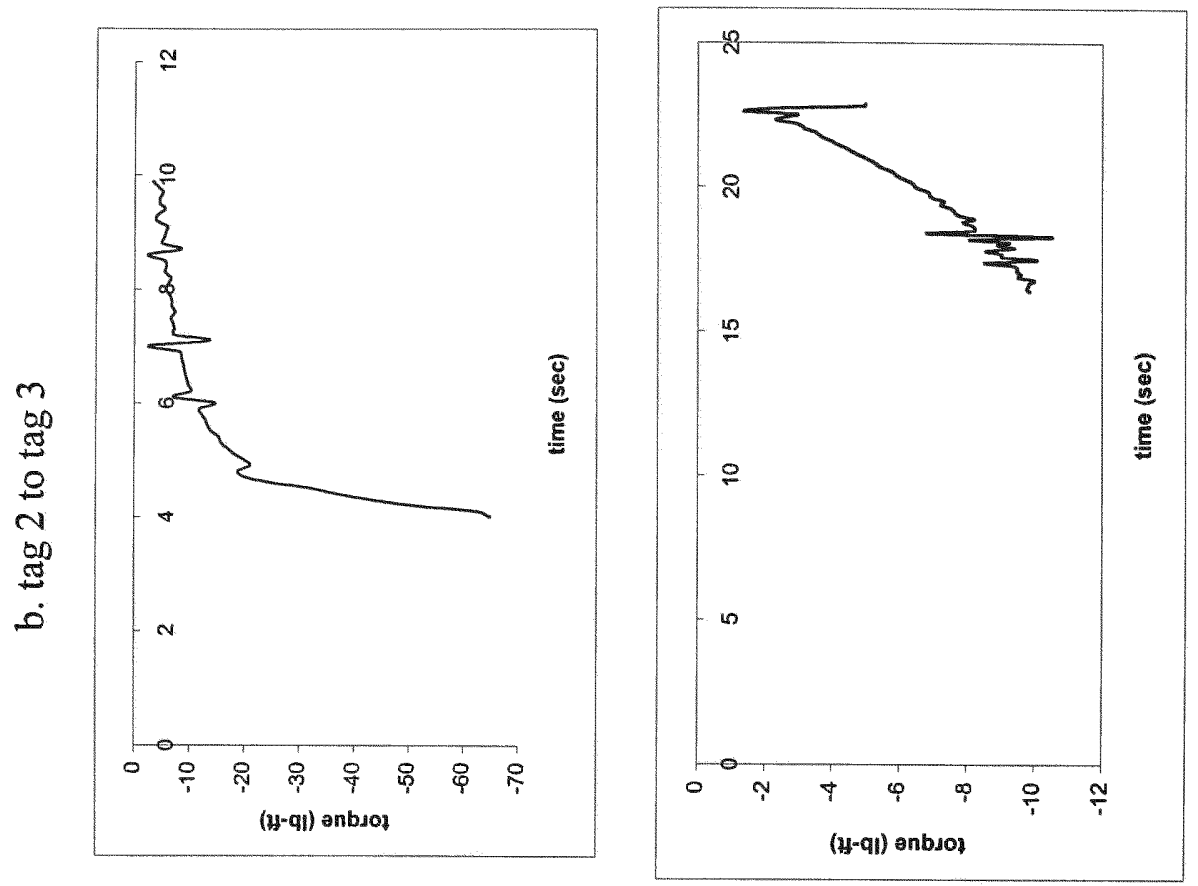

\begin{tabular}{l}
$n$ \\
00 \\
$\Xi$ \\
0 \\
0 \\
0 \\
$\Xi$ \\
\hline
\end{tabular}

ก

雚

N
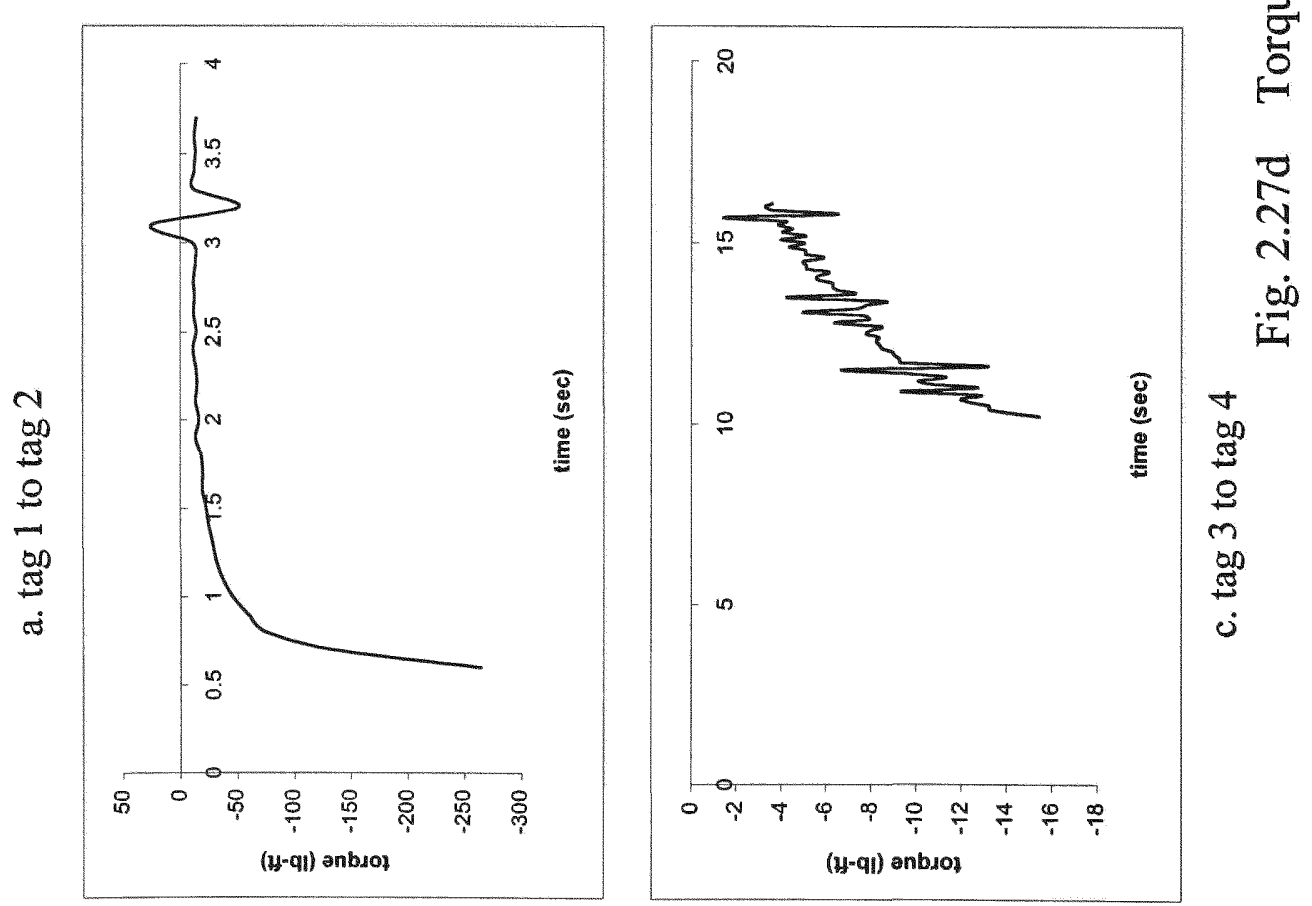

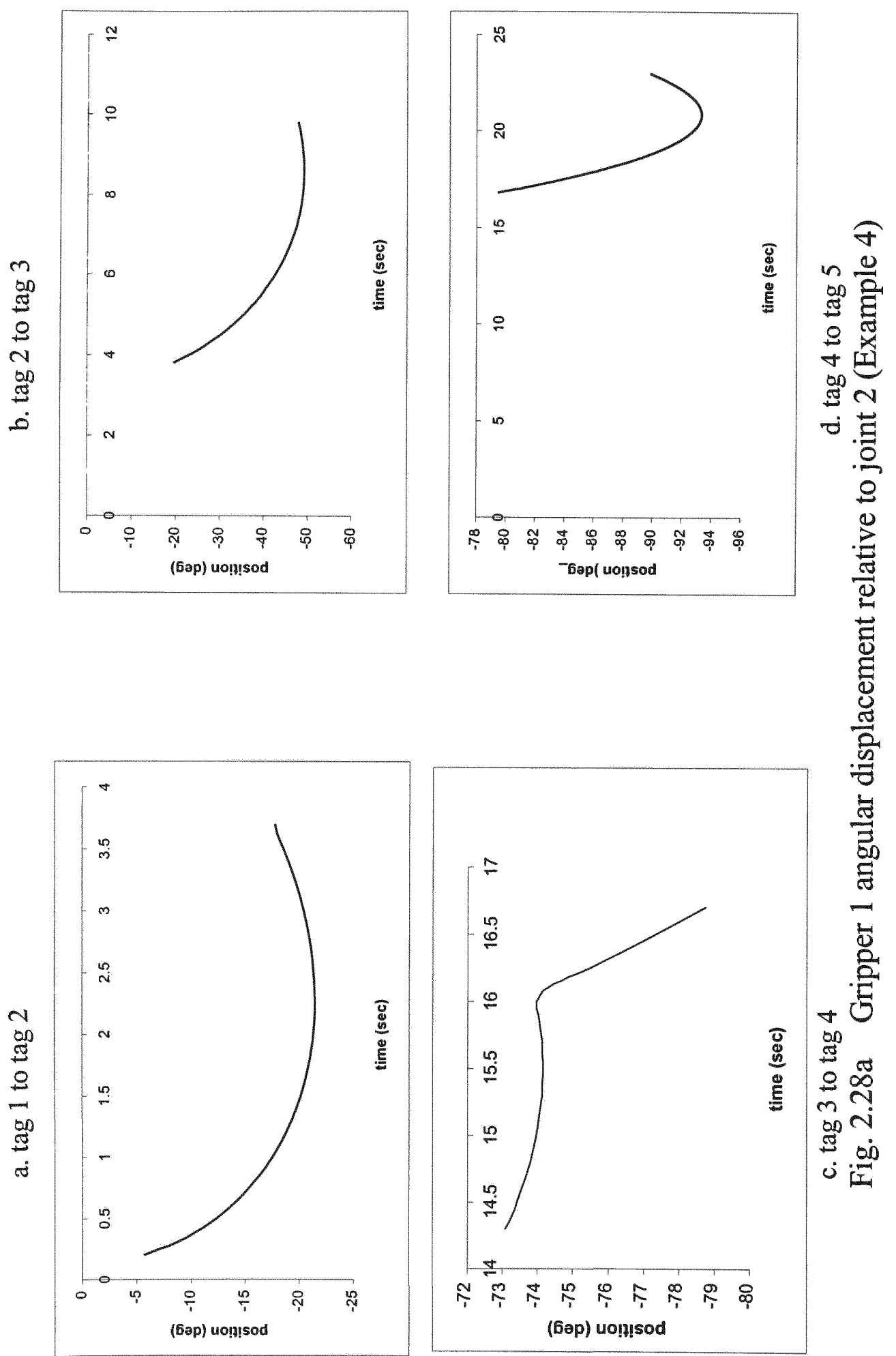
Example 4: Gripper 2 grasps the structural member in the tank and gripper 1 moves

from $t 1$ to $t 5$ to grasp the same structural member.

Data of angular displacement, velocity and acceleration, and joint torque have been collected from Tele/IGRIP through simulated results as shown in Figs. 2.14 a to e. The end-effector (gripper 1) takes 23 seconds to move from its initial position tl to final position $t 5$ by passing through intermediate tag points $t 2, t 3$, $t 4$. It moves with a steady speed of 20 inches per second and carrying a payload of $110 \mathrm{lb}$. To analyze the motion kinematics between straight line trajectory segments, each graph is divided into four figures.

Angular displacement of gripper 1 with respect to joint 2 is shown in Fig. 2.28a. As seen in the graphs, angular displacement is increasing in magnitude most of the time and always in the permissible limits $\left( \pm 135^{\circ}\right)$. Angular velocity of gripper 1, Fig. 2.28b, slows down gradually and varies in a linear pattern at the end of its motion. Discontinuous points must be always interpolated by joining the adjacent points to follow the general trend of the curve. Fig. $2.28 \mathrm{c}$ shows the angular acceleration of gripper 1 . It decelerates to the end tag point in each trajectory segment. Discontinuous points ought to be interpolated in this case too. Torque of joint 2 associated with gripper 1 is given Fig. 2.28d. For the first two trajectory segments, i.e. when it moves from $t 1$ to $t 2$ and $t 2$ to $t 3$, joint torque decreases in magnitude. However, it increases in the last two trajectory segments. Interpolation has to be done for discontinuous points in the curves. Similar interpretations can be made for joints $3,3^{\prime}$, and $2^{\prime}$ by referring to Figs. $2.30 \mathrm{a}$ to d, Figs. 2.31a to d, and Figs. 2.32a to d, respectively. The torque applied to the structural member, exerted by gripper 2, is identical to the torque of joint 2', Fig. $2.32 \mathrm{~d}$. 
The VTM elevates gripper 1 from $56.95 "$ to 66.25 " so that the RTD system can grasp the same structural member. Angular displacement of the VTM is shown in Fig. 2.29a. It increases linearly with respect to time. The graph in Fig. $2.29 \mathrm{~b}$ shows the angular velocity of the VTM. It is constant for the motions between the first three trajectory segments. Moreover, angular acceleration between the last trajectory segment varies as a step function. Angular accelerations of the VTM are about zero degree per second square for the entire motion as in shown Fig. $2.29 \mathrm{c}$ since the angular velocities are constants. Torque of the VTM is also represented in Fig 2.29d. 

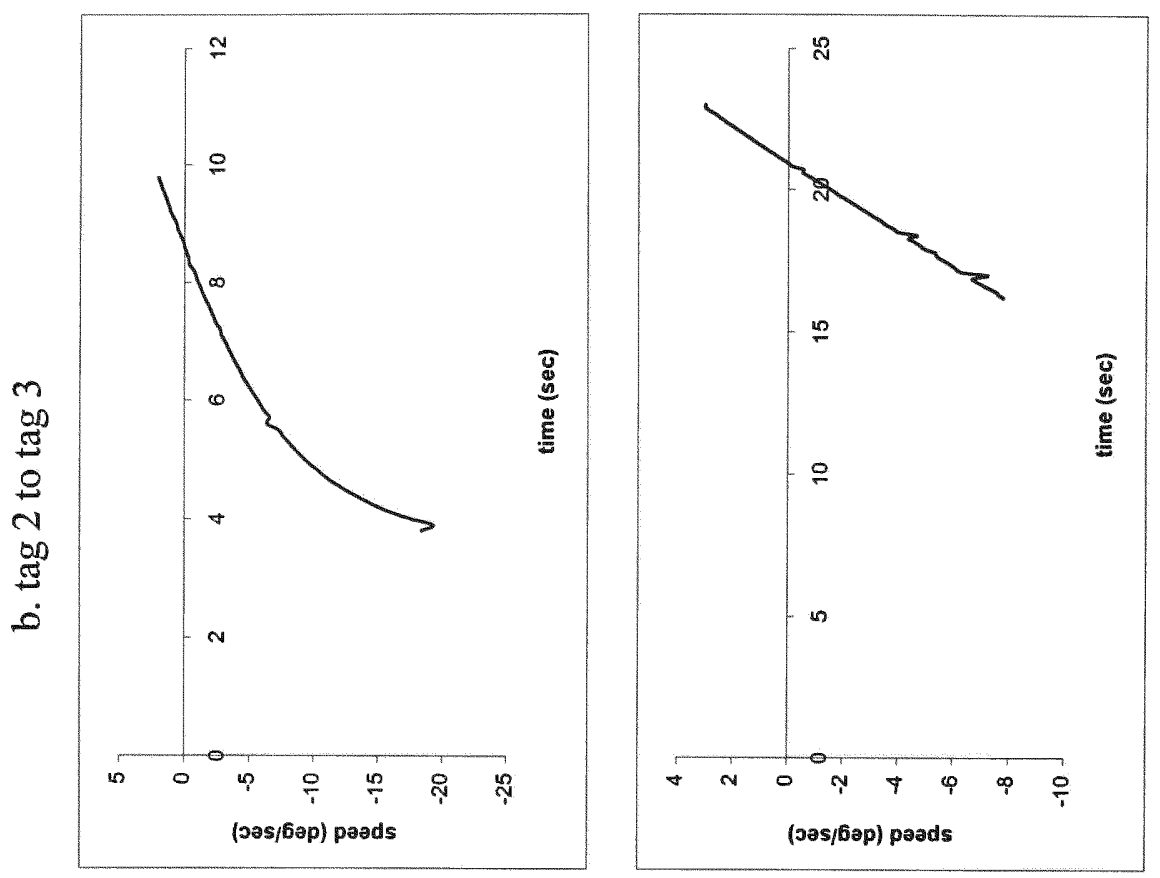

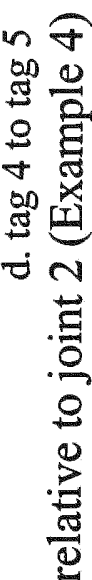
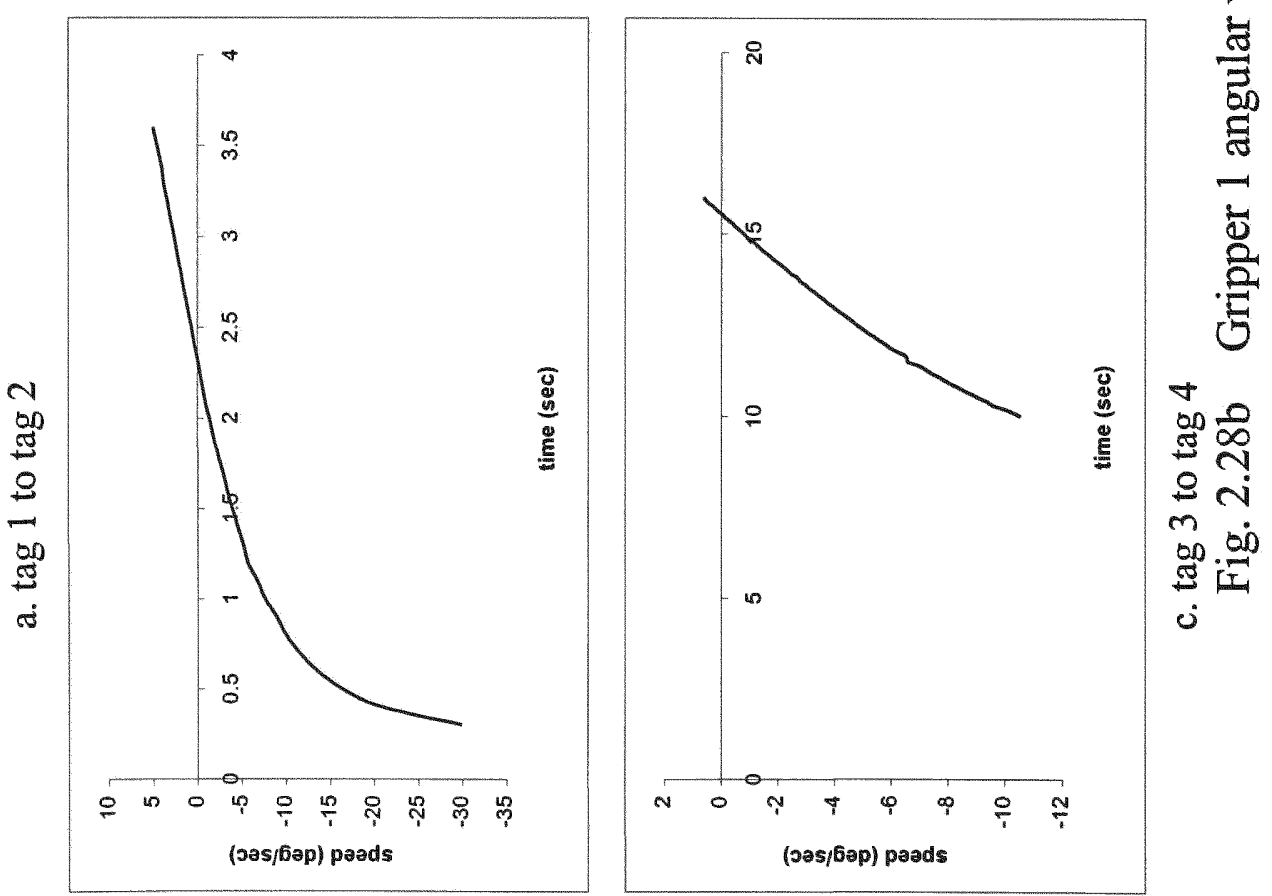

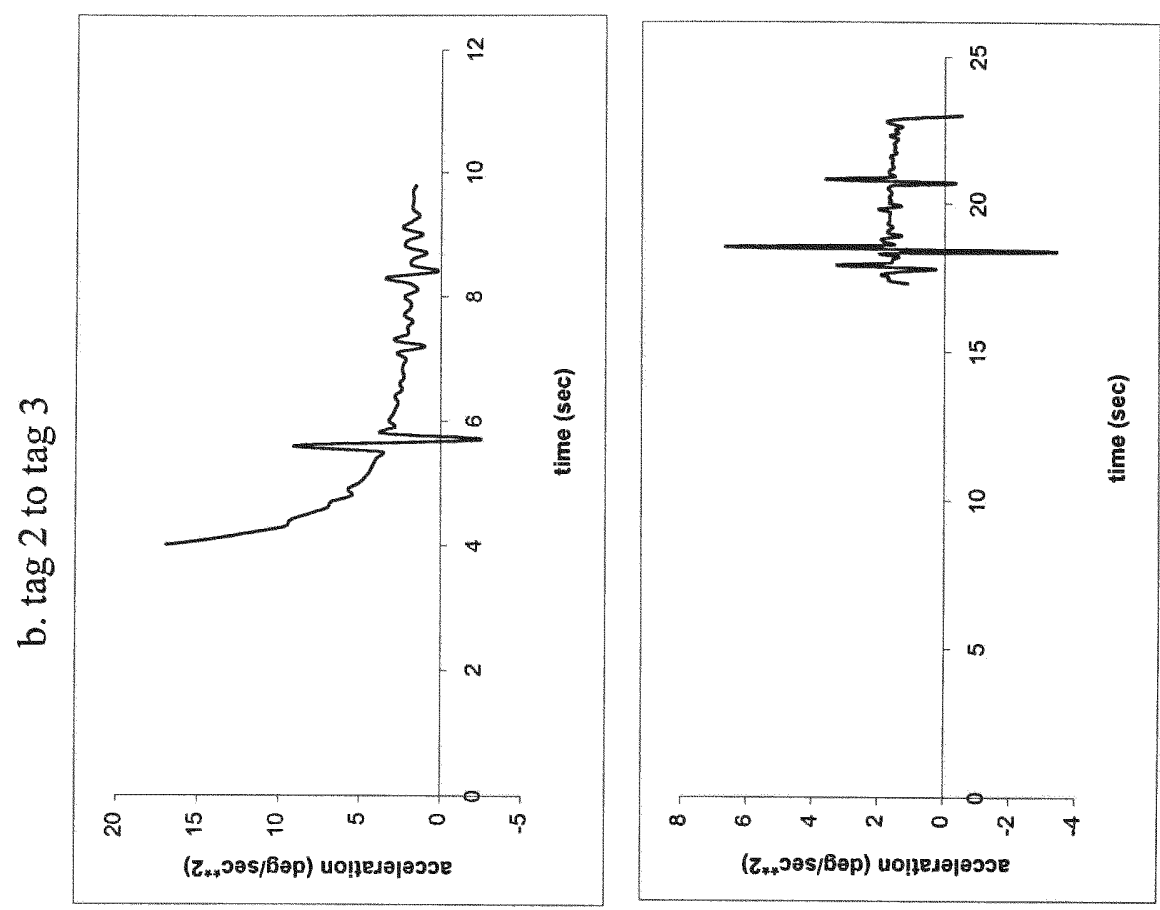

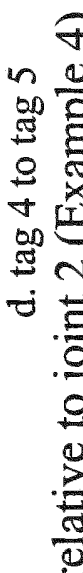
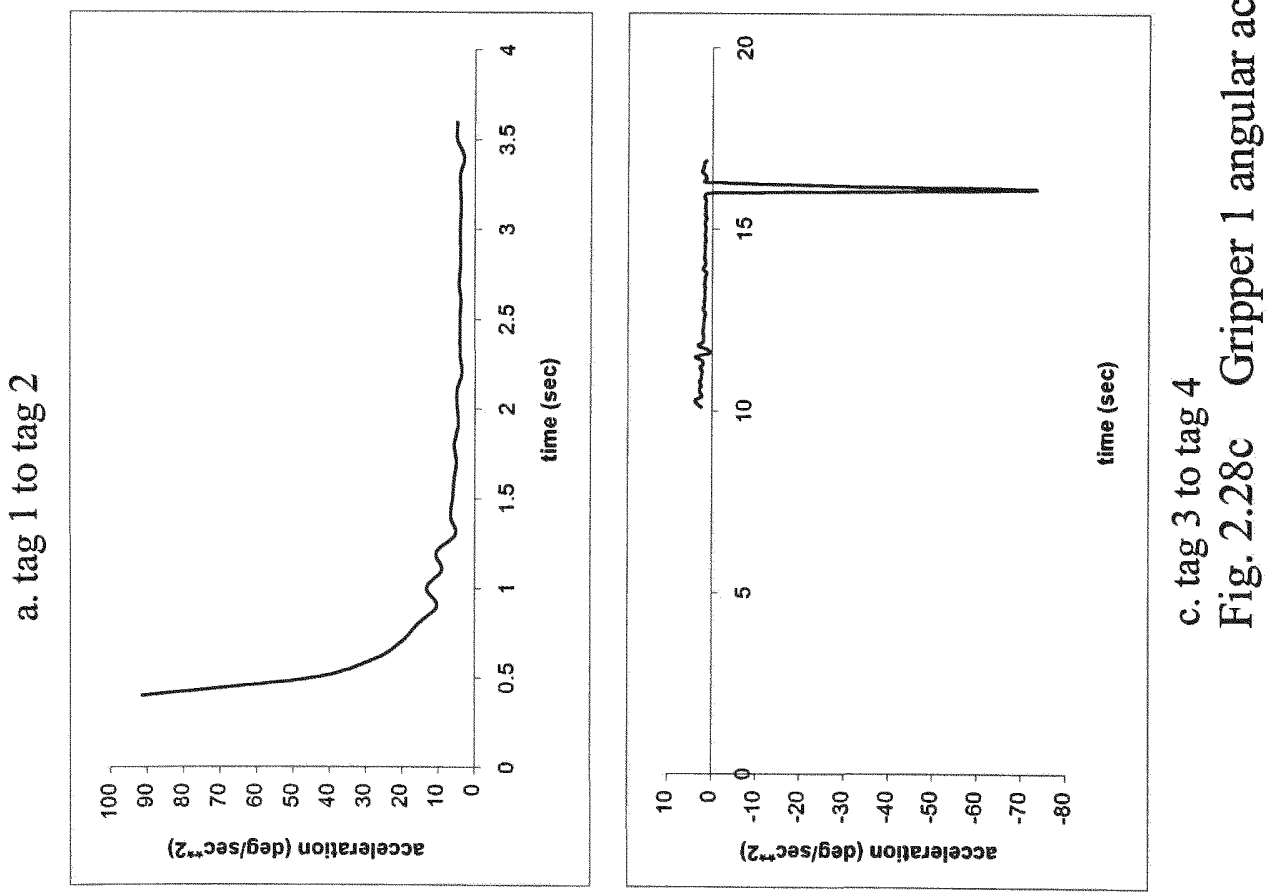

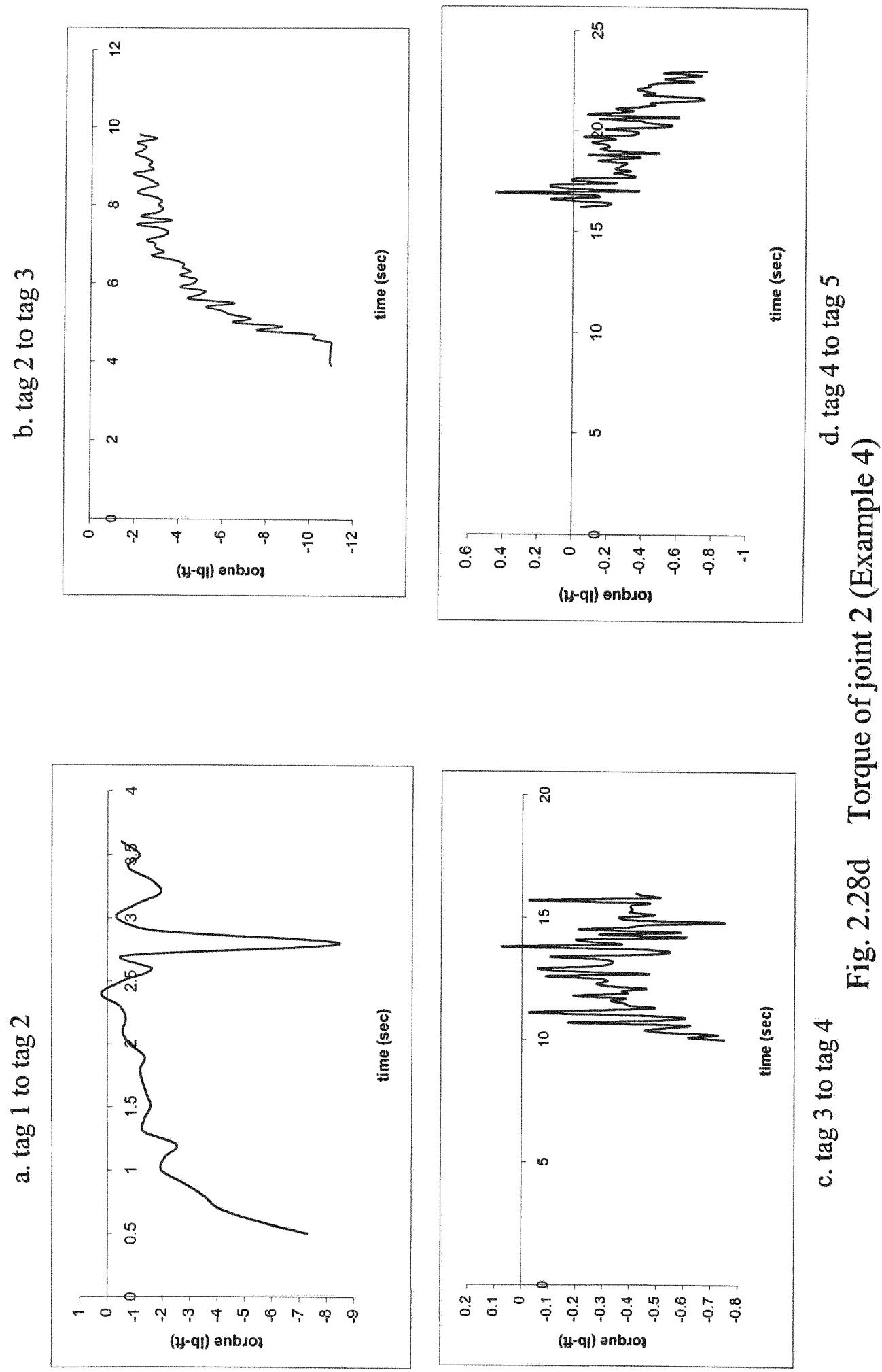

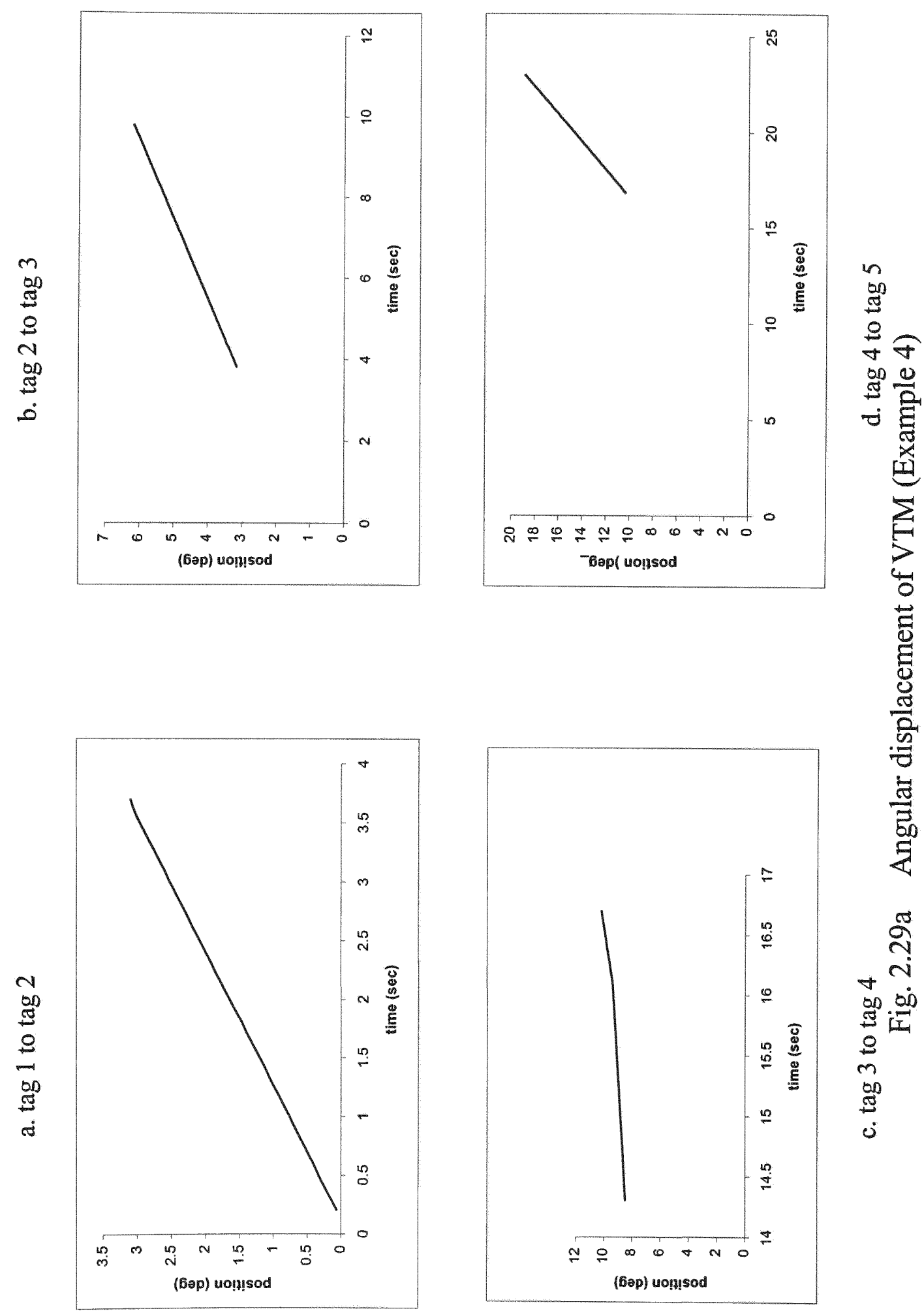

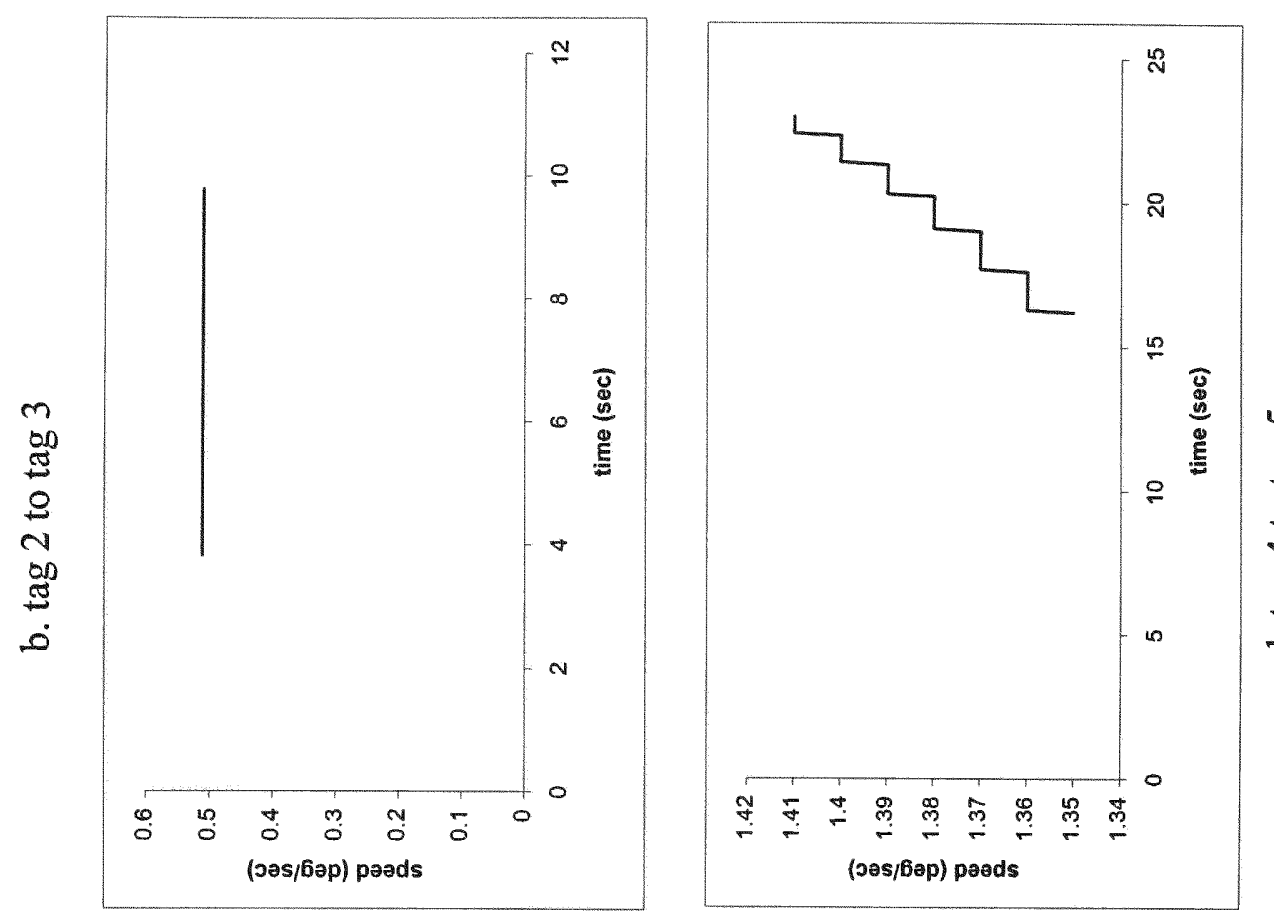

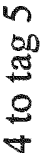

\%

ठํㅡㄹ

灵
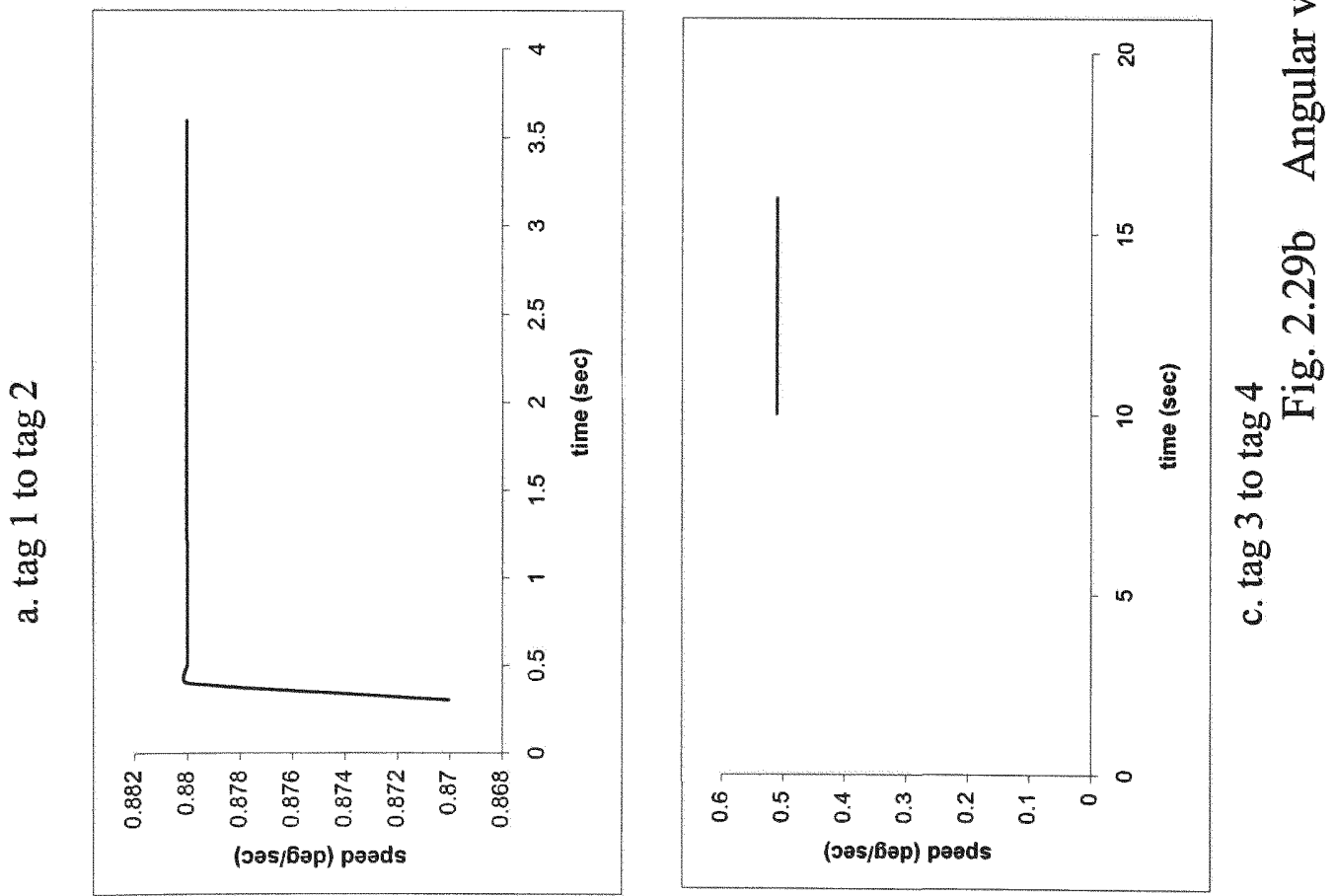

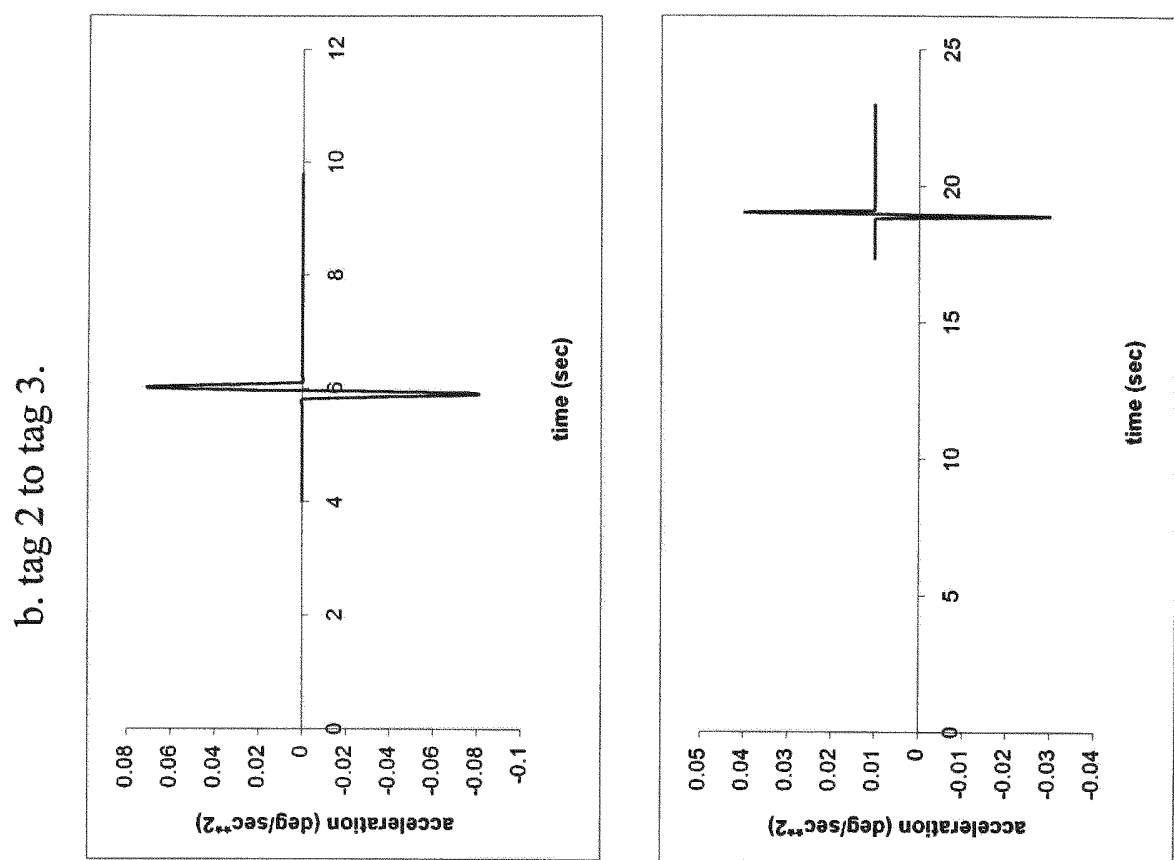

n

$\nabla$

(⿻)

\$

ठ

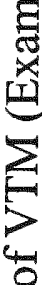
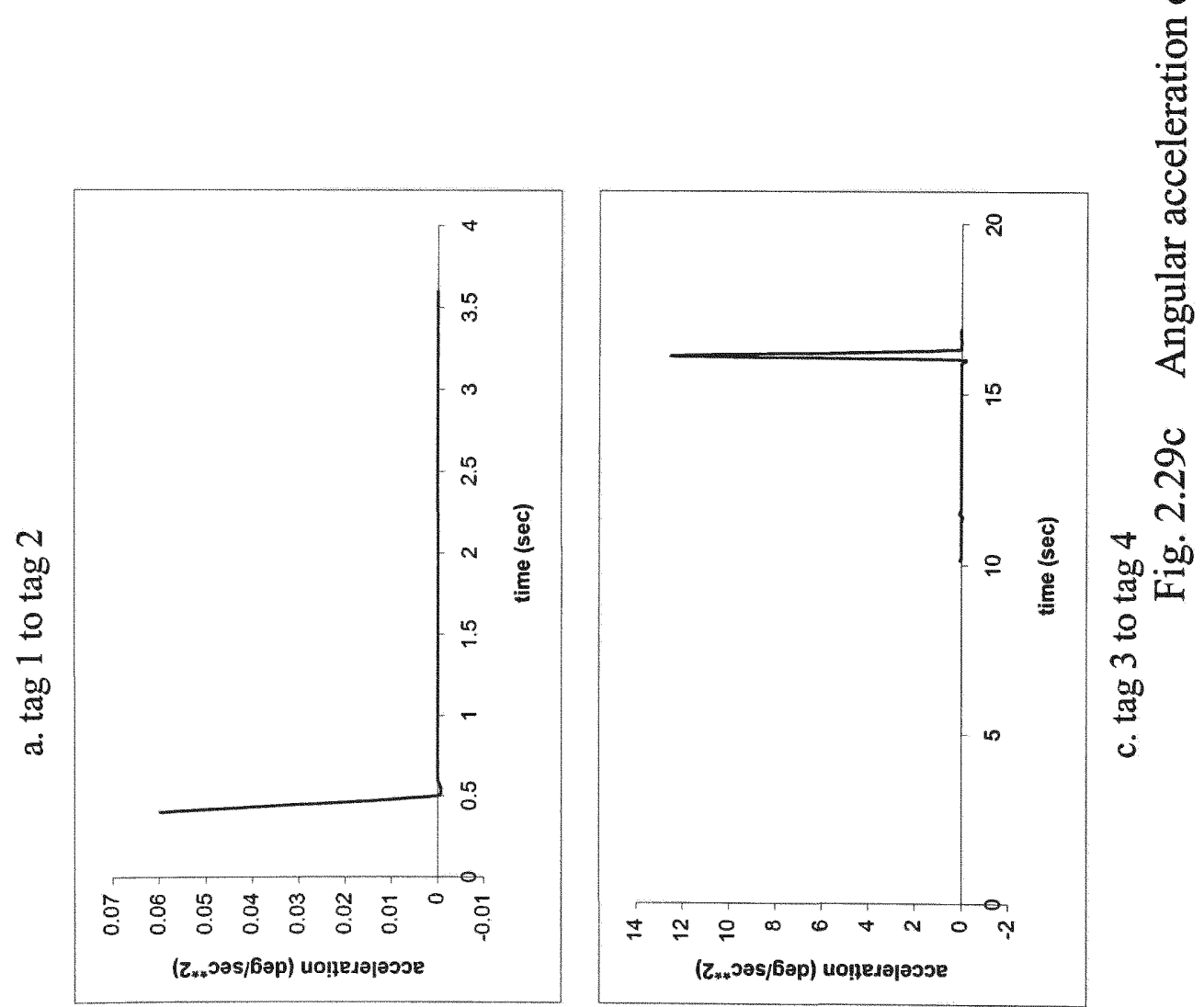

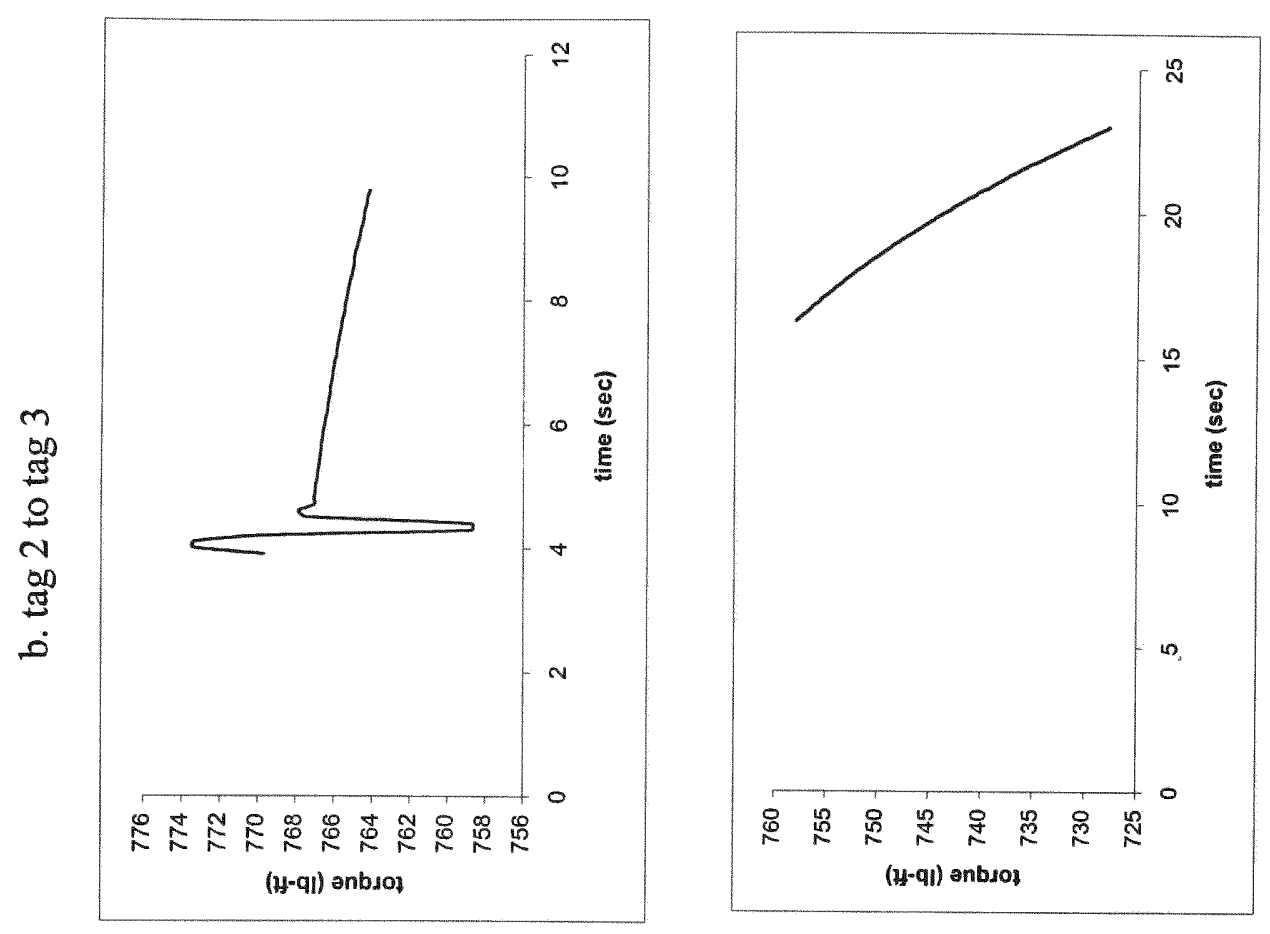

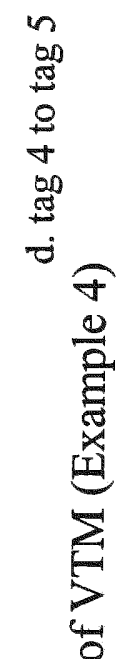
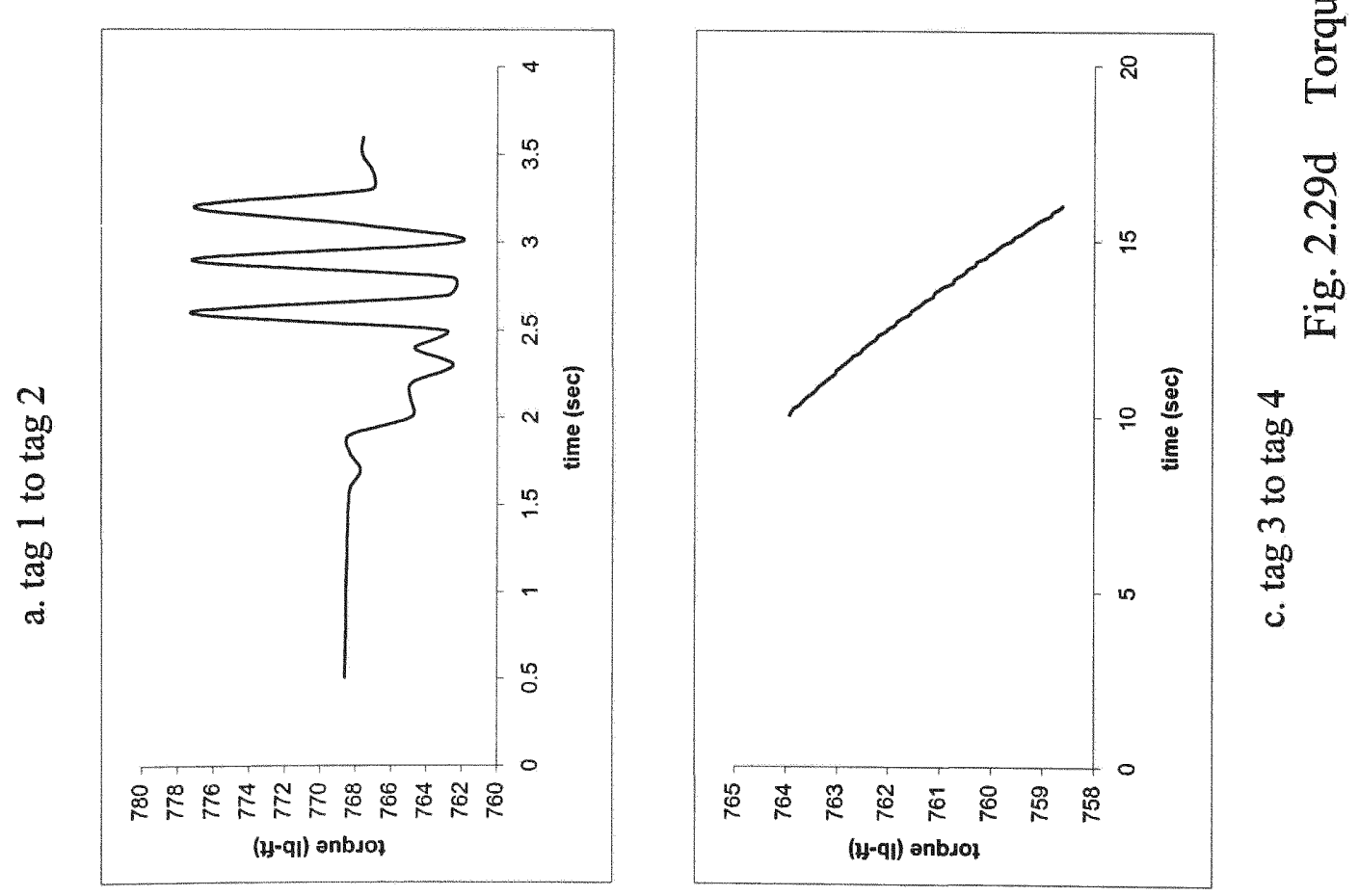

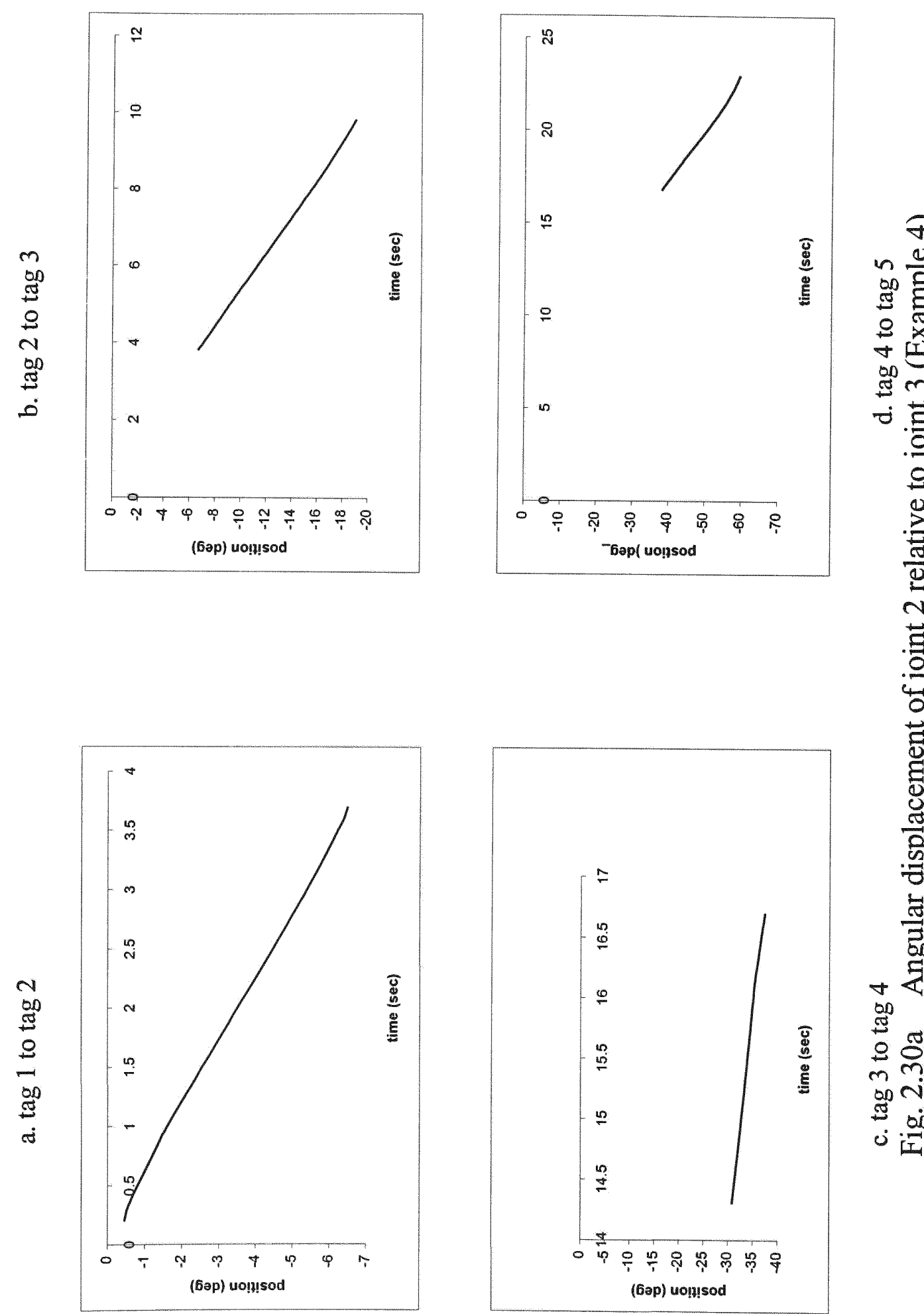

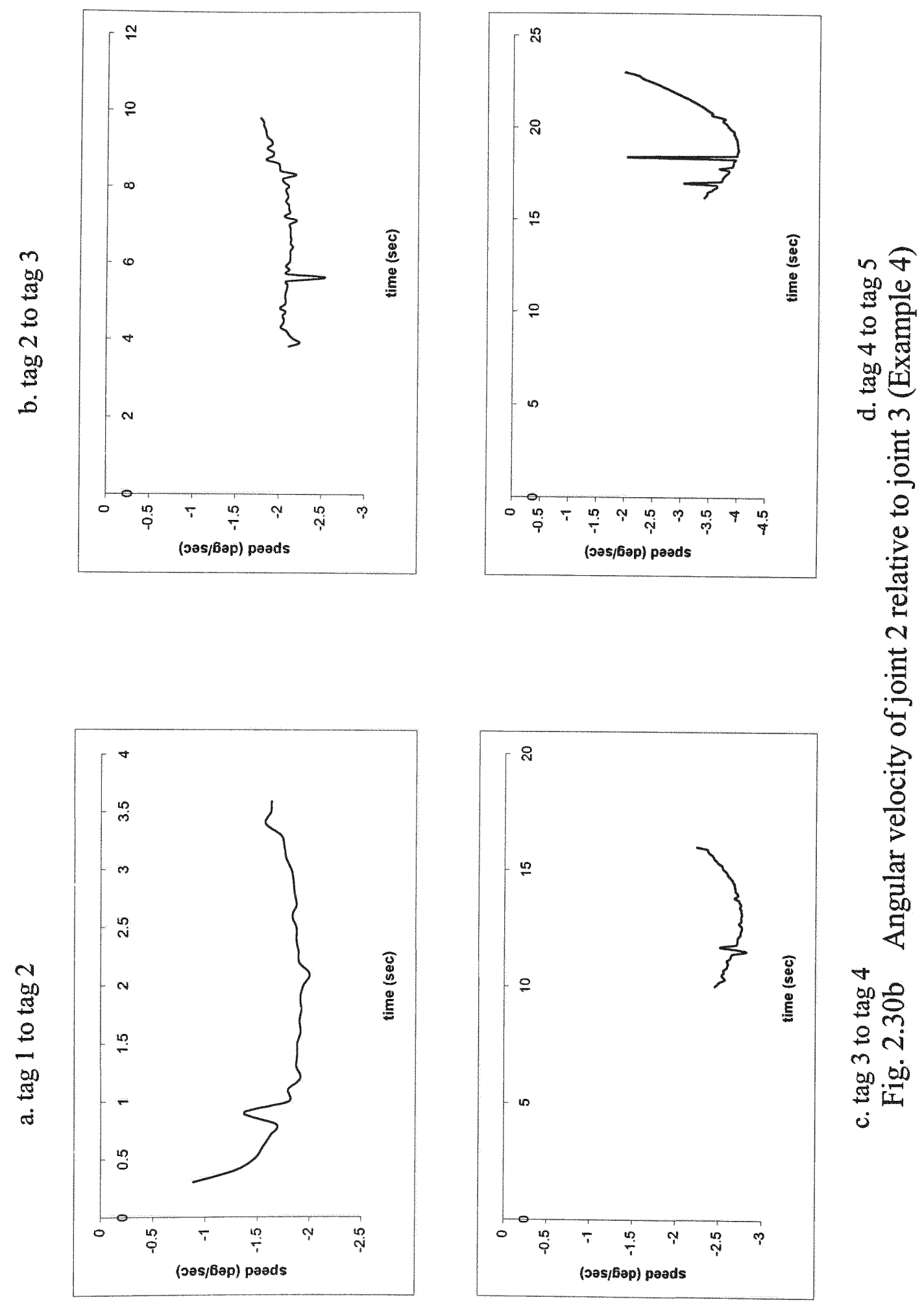

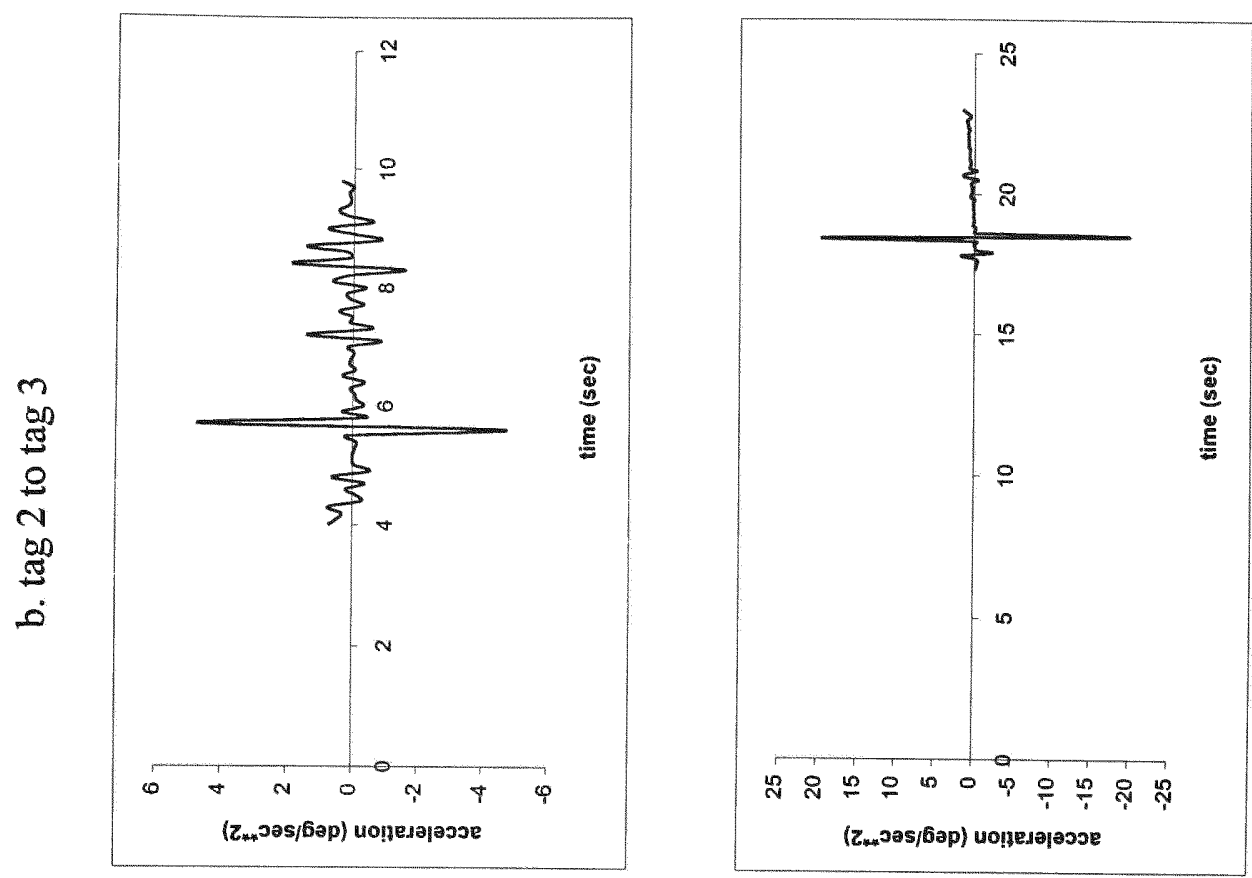

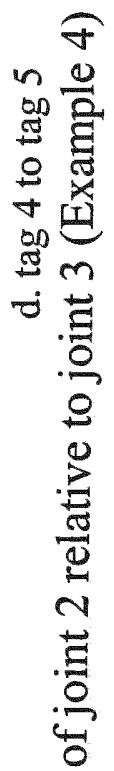
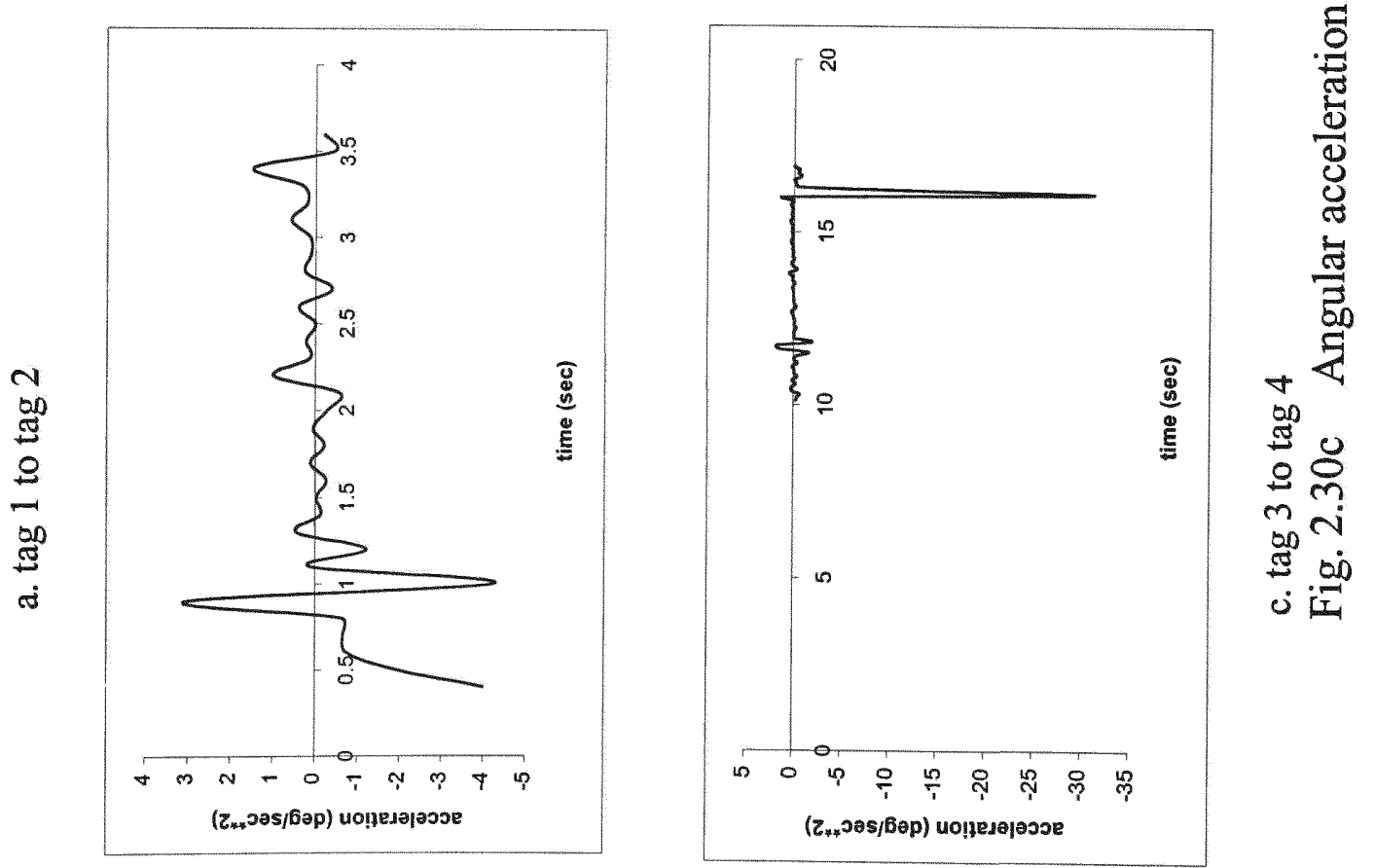

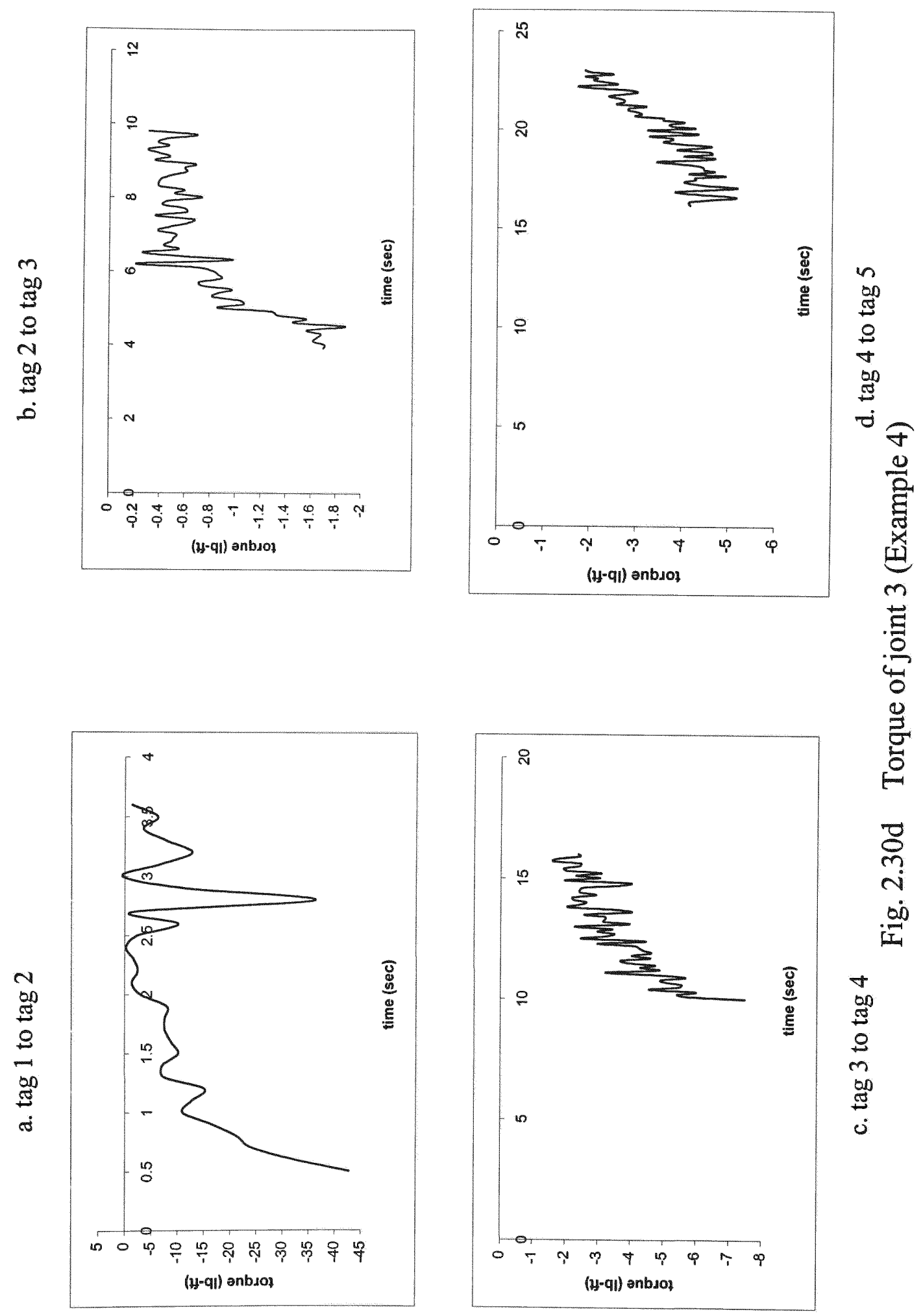

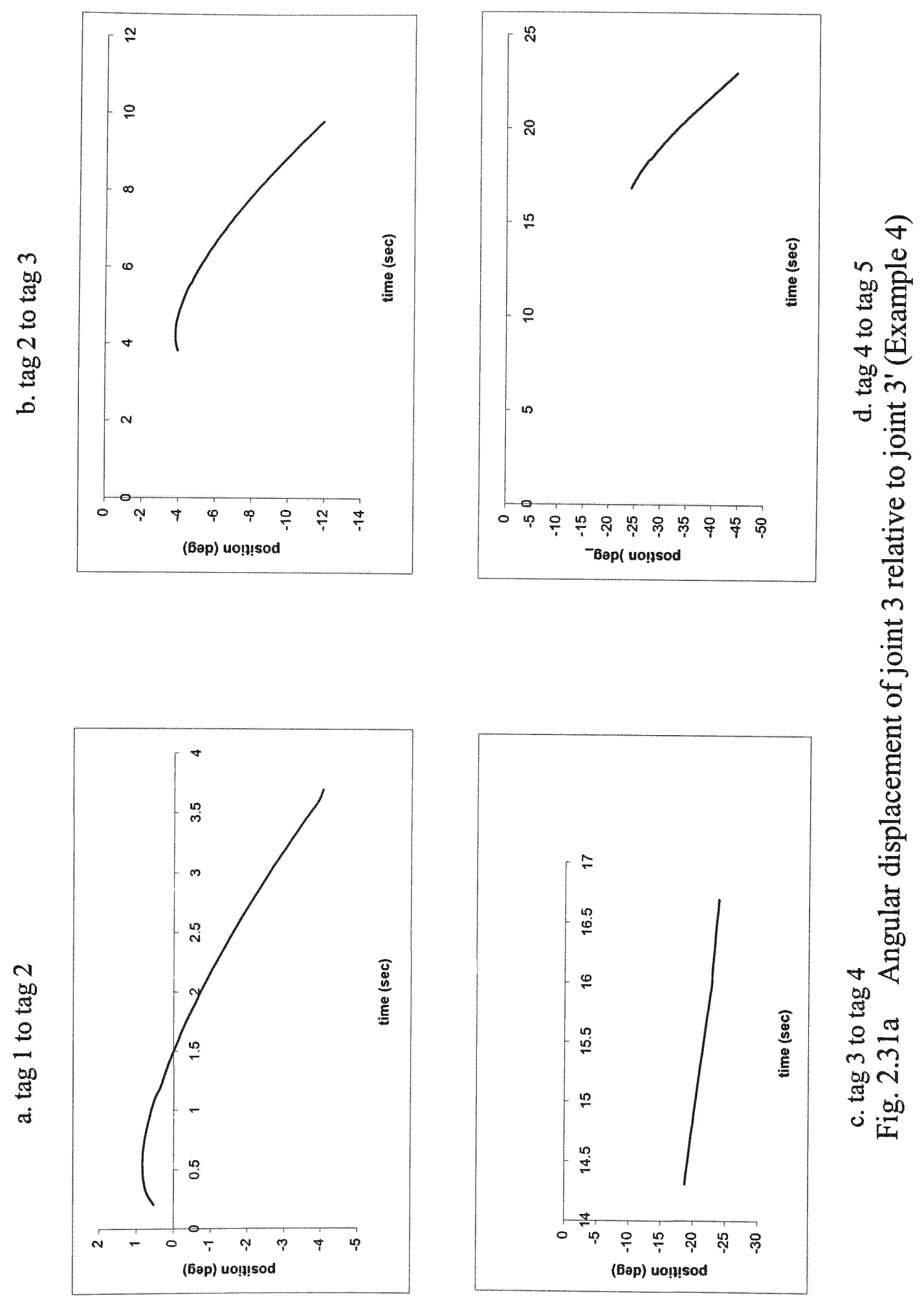

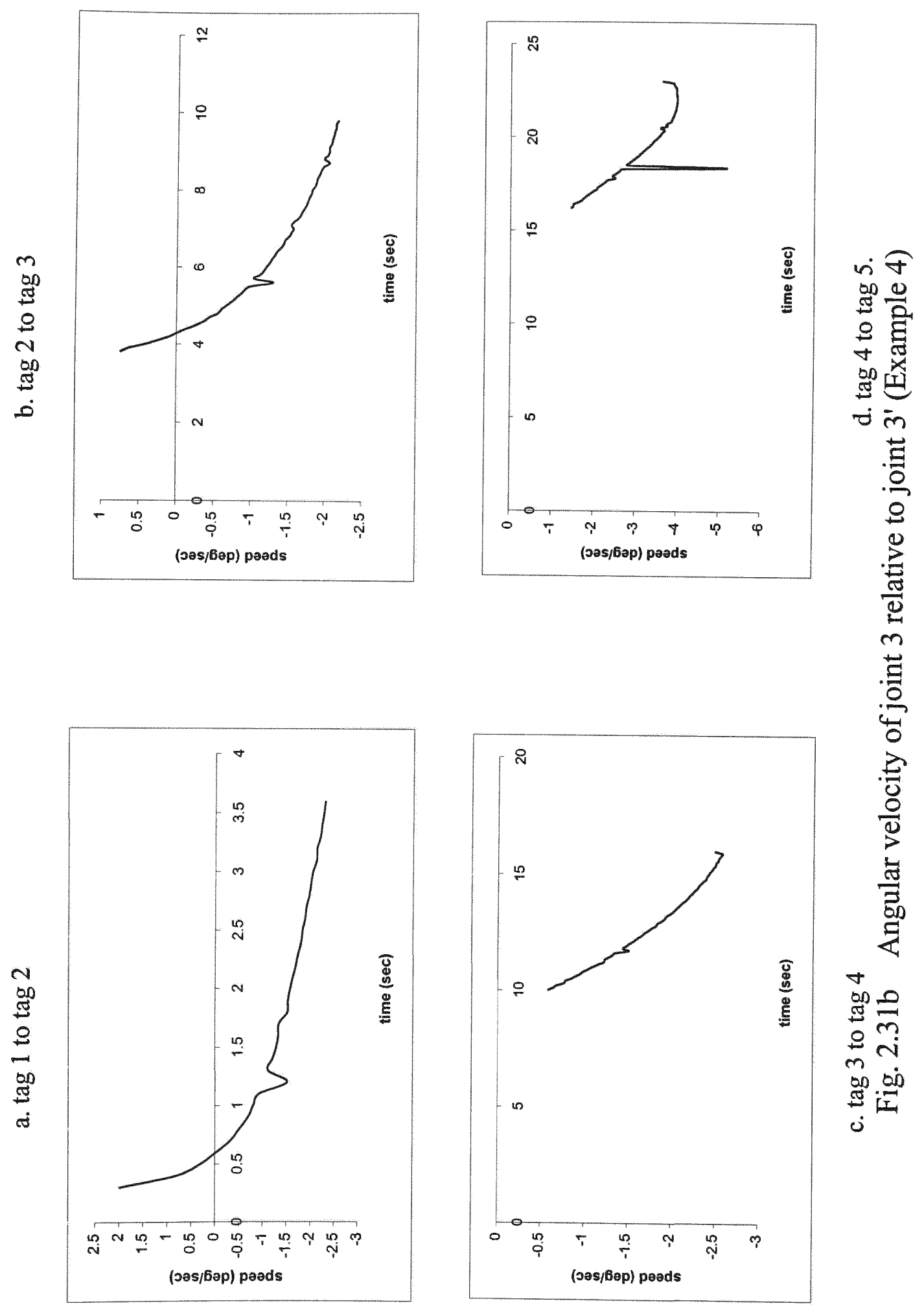

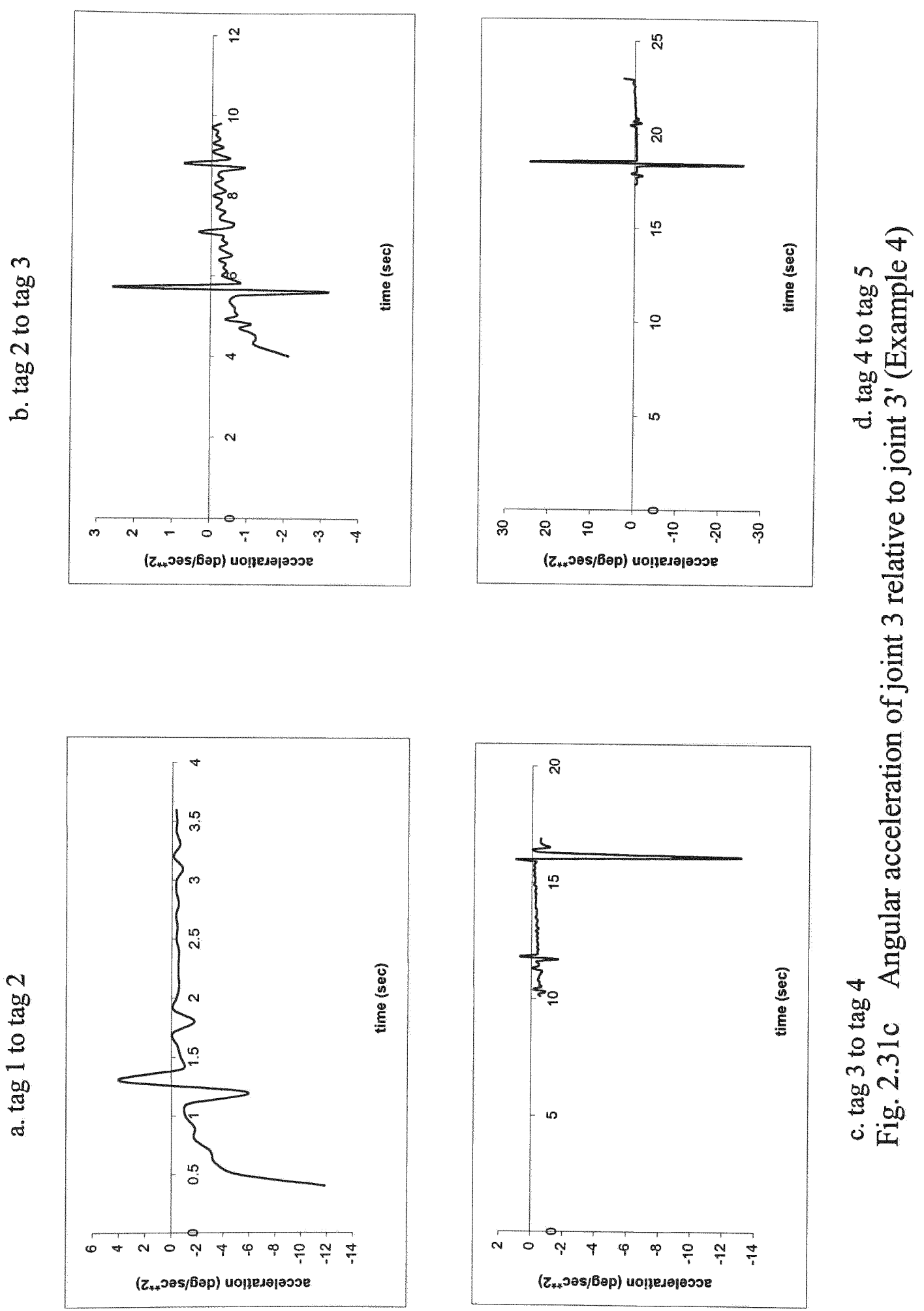

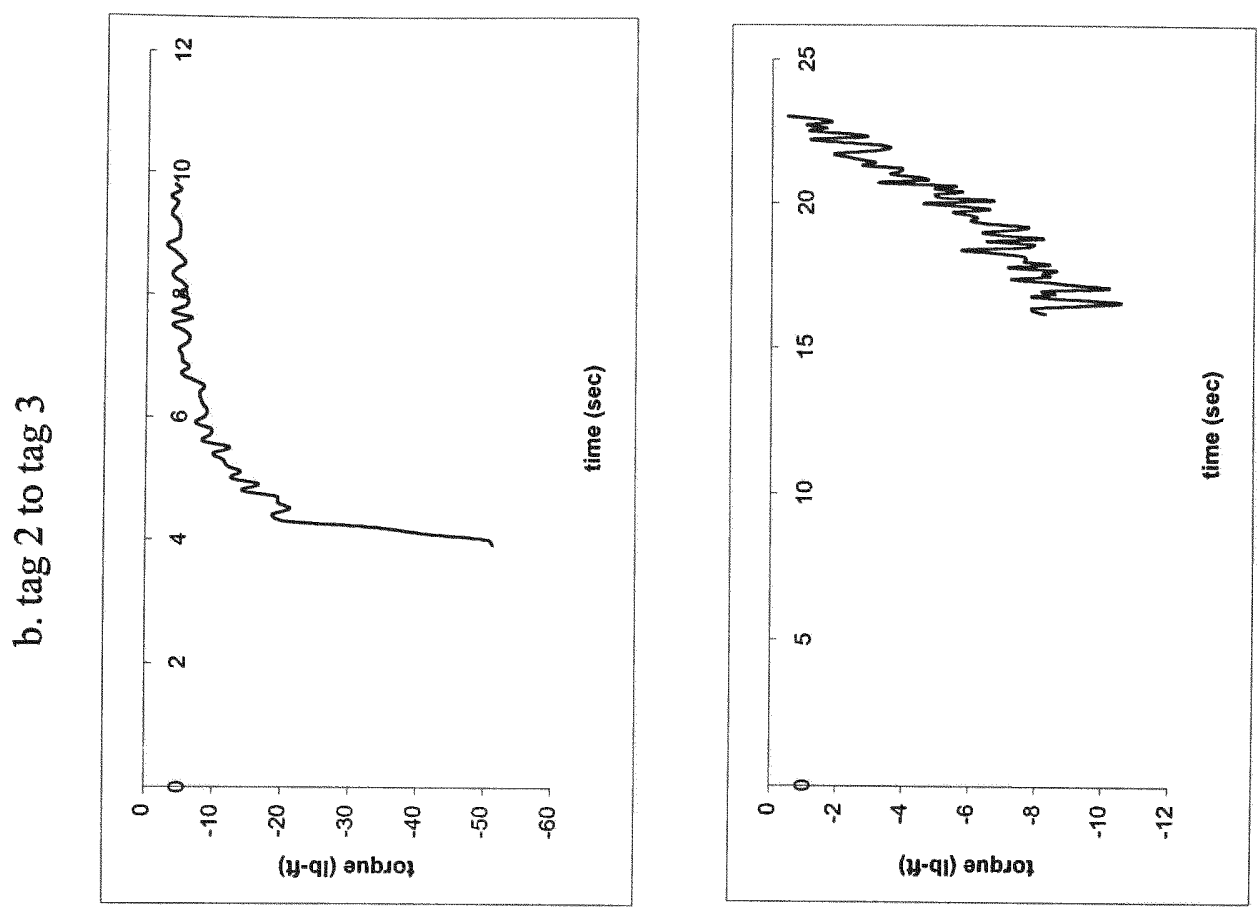

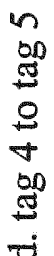

$\nabla$

है

in

若
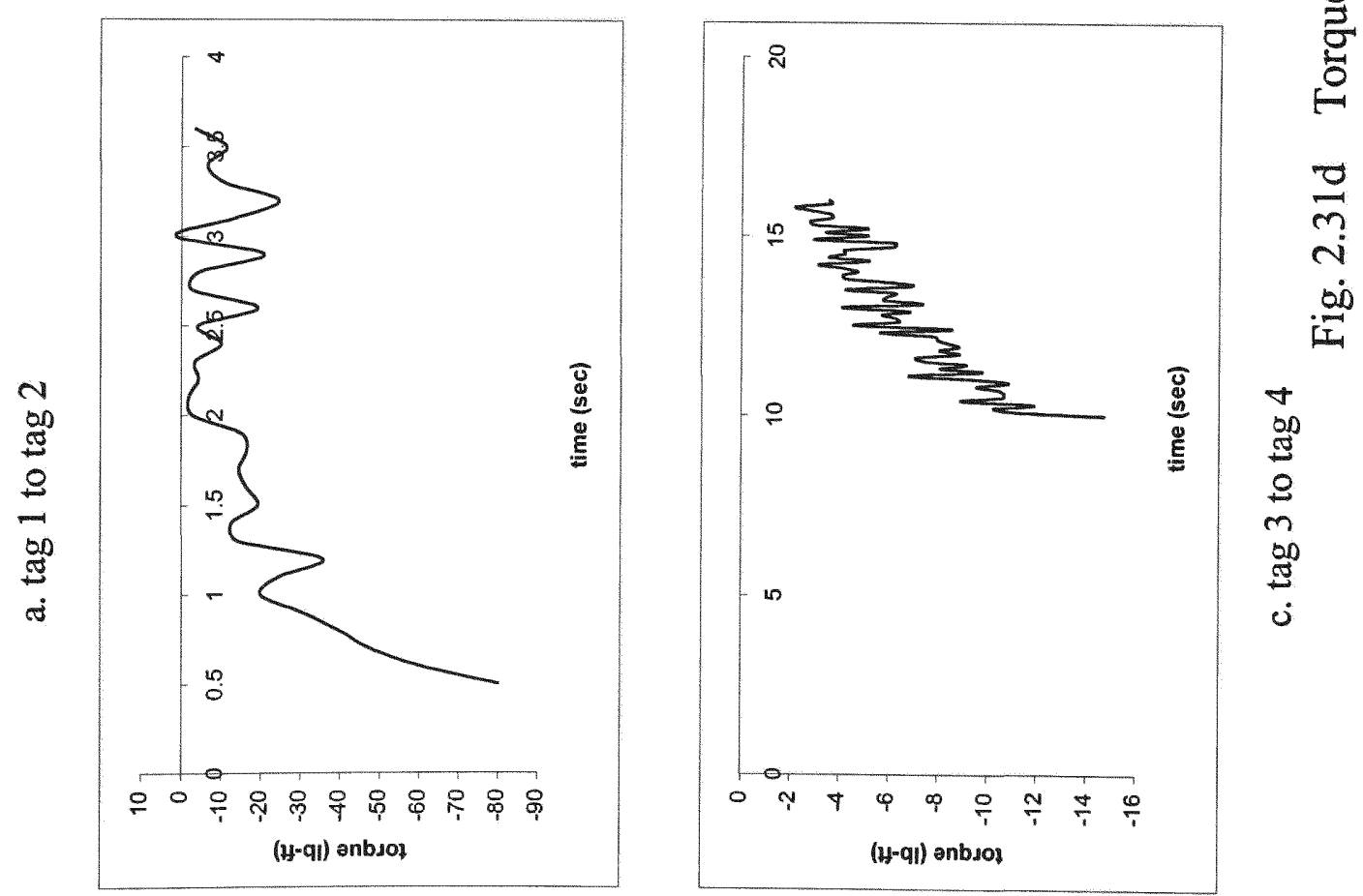

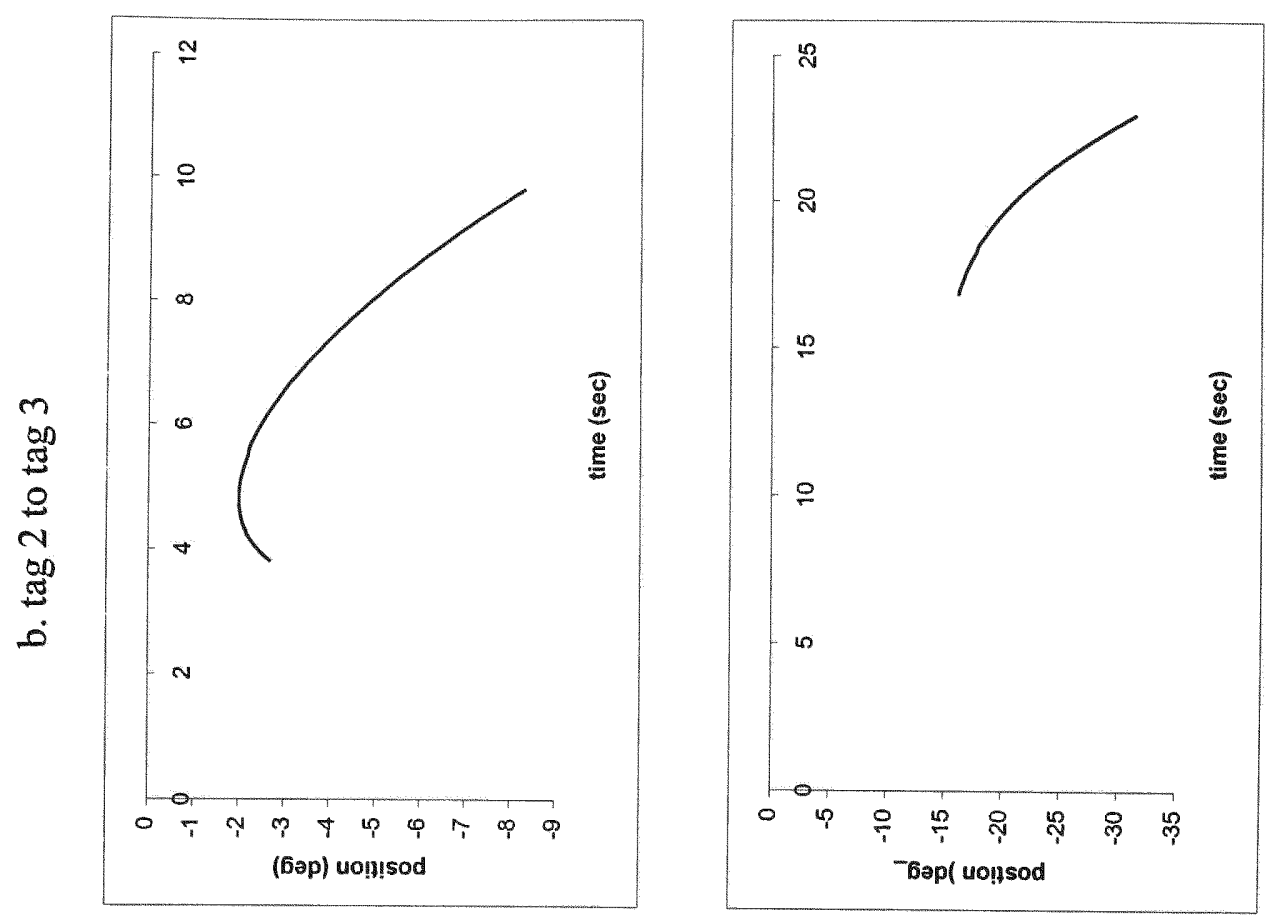

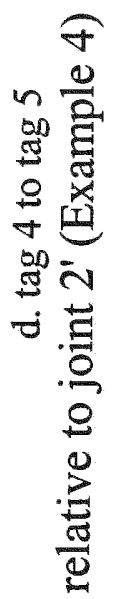
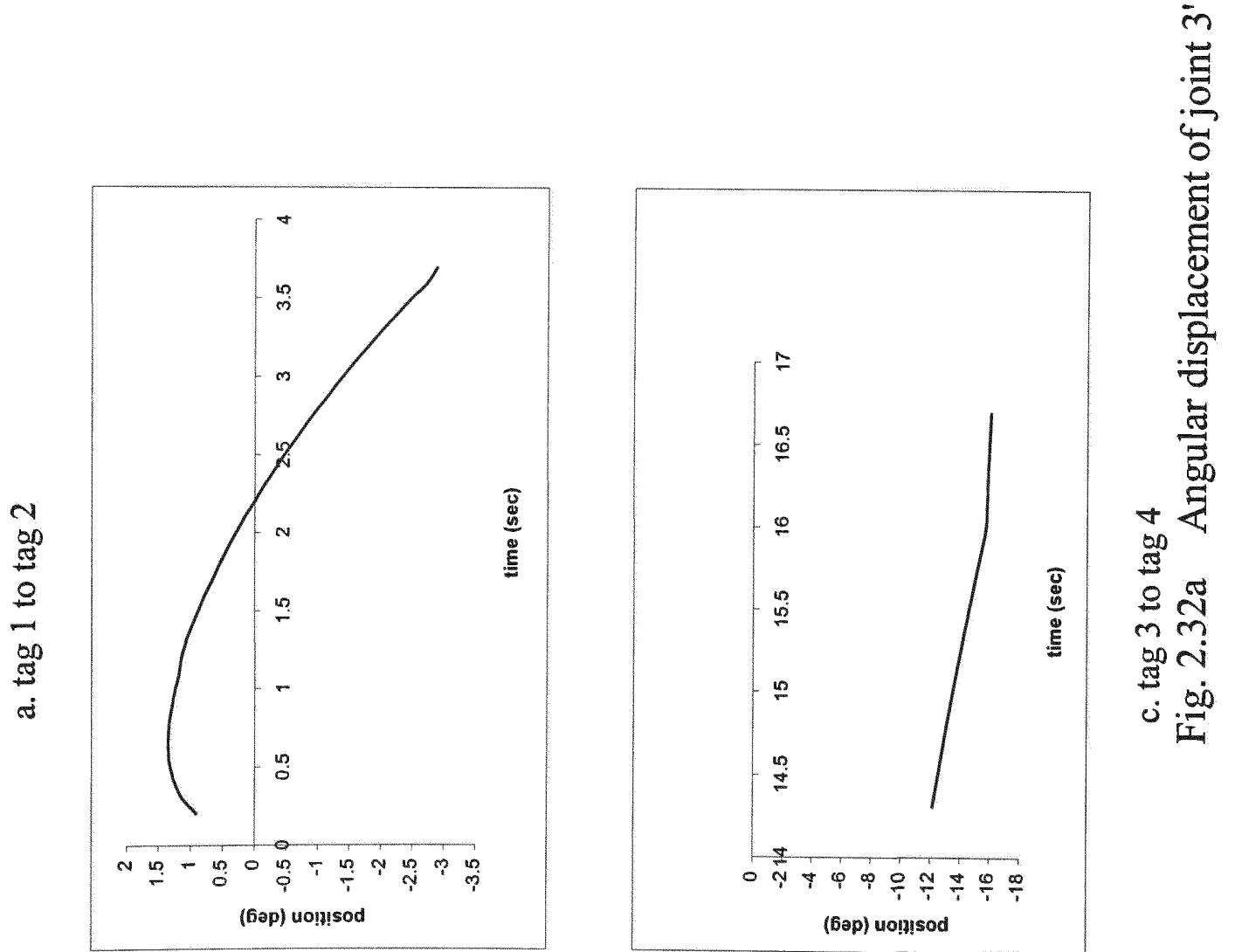

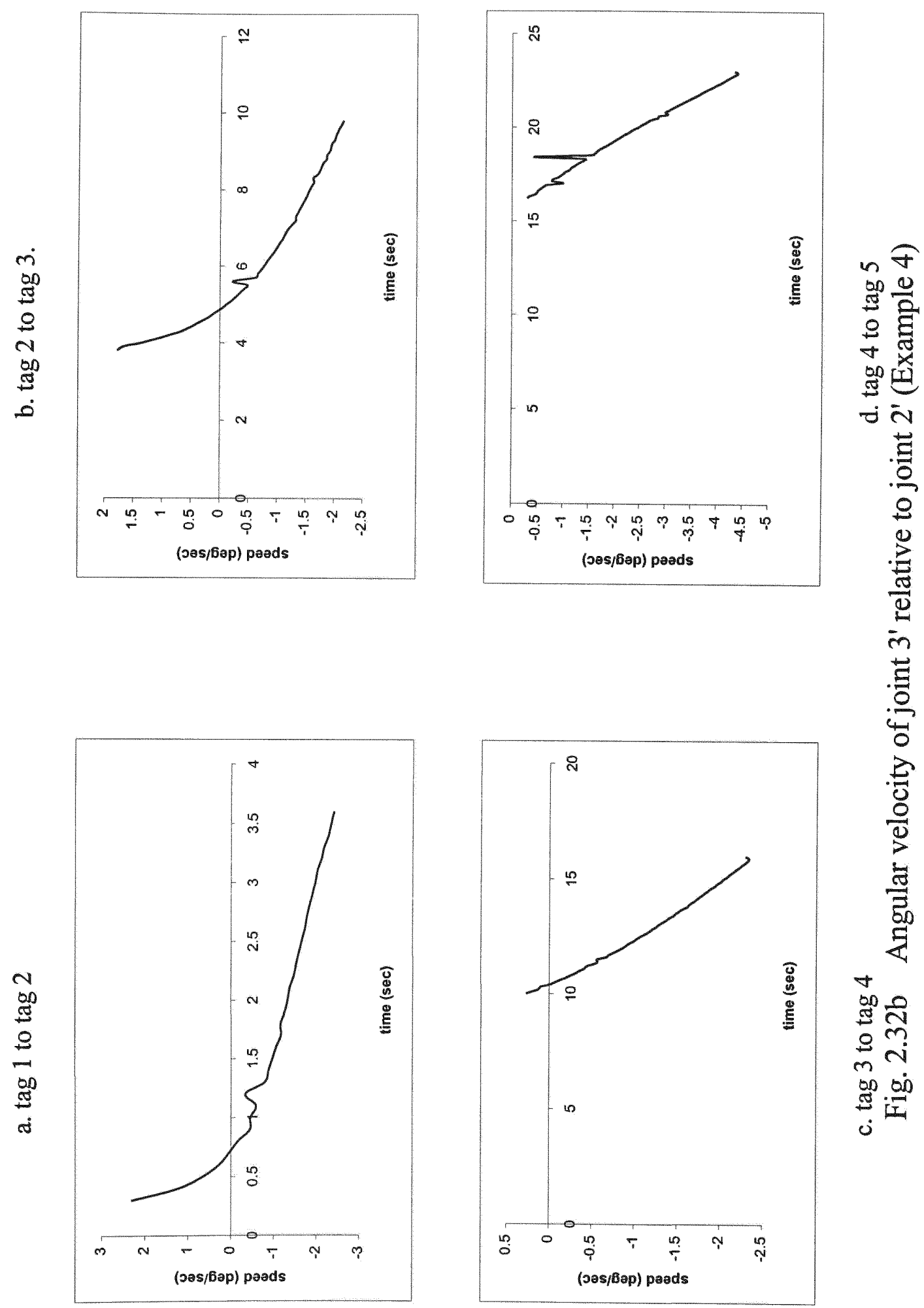

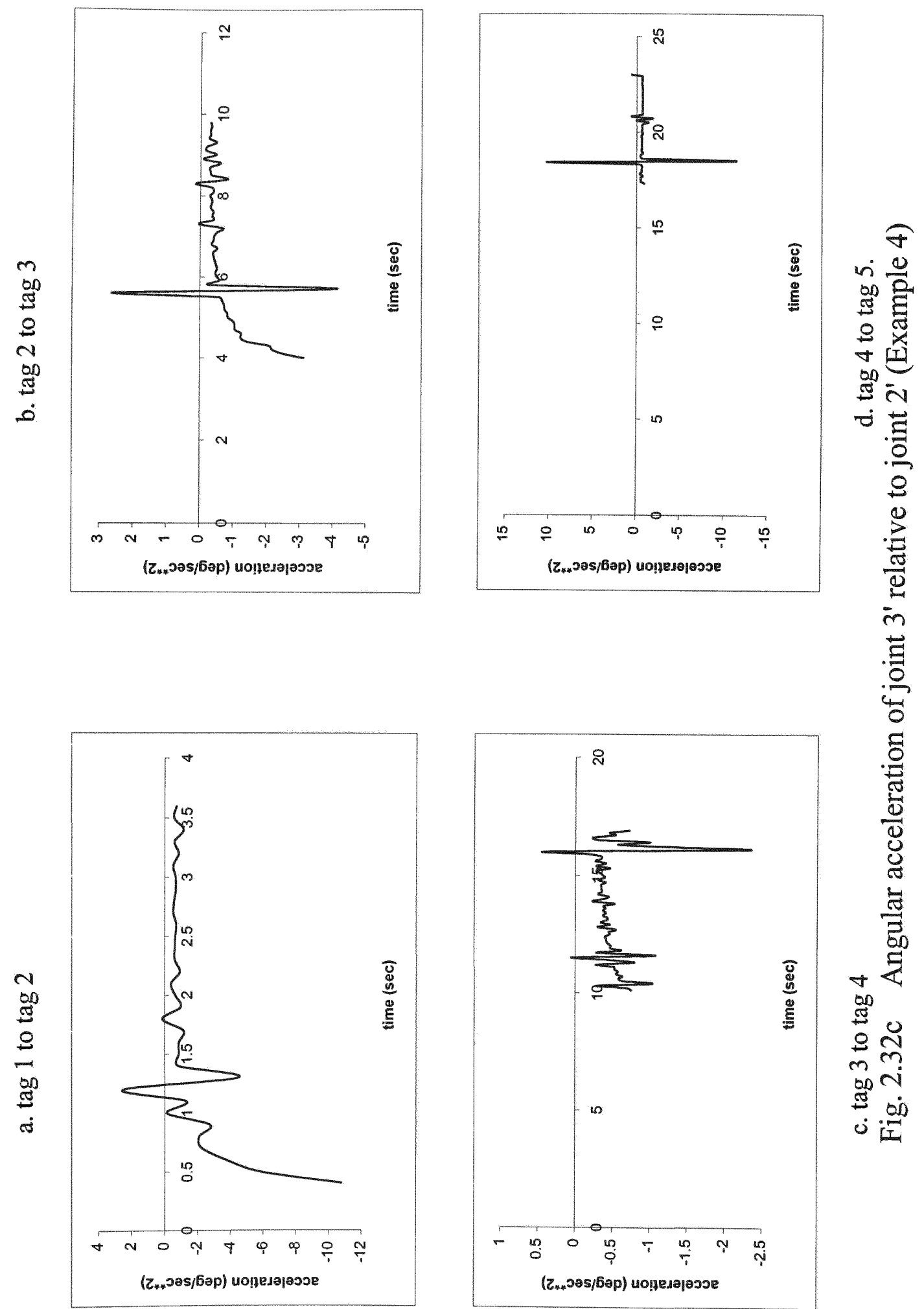

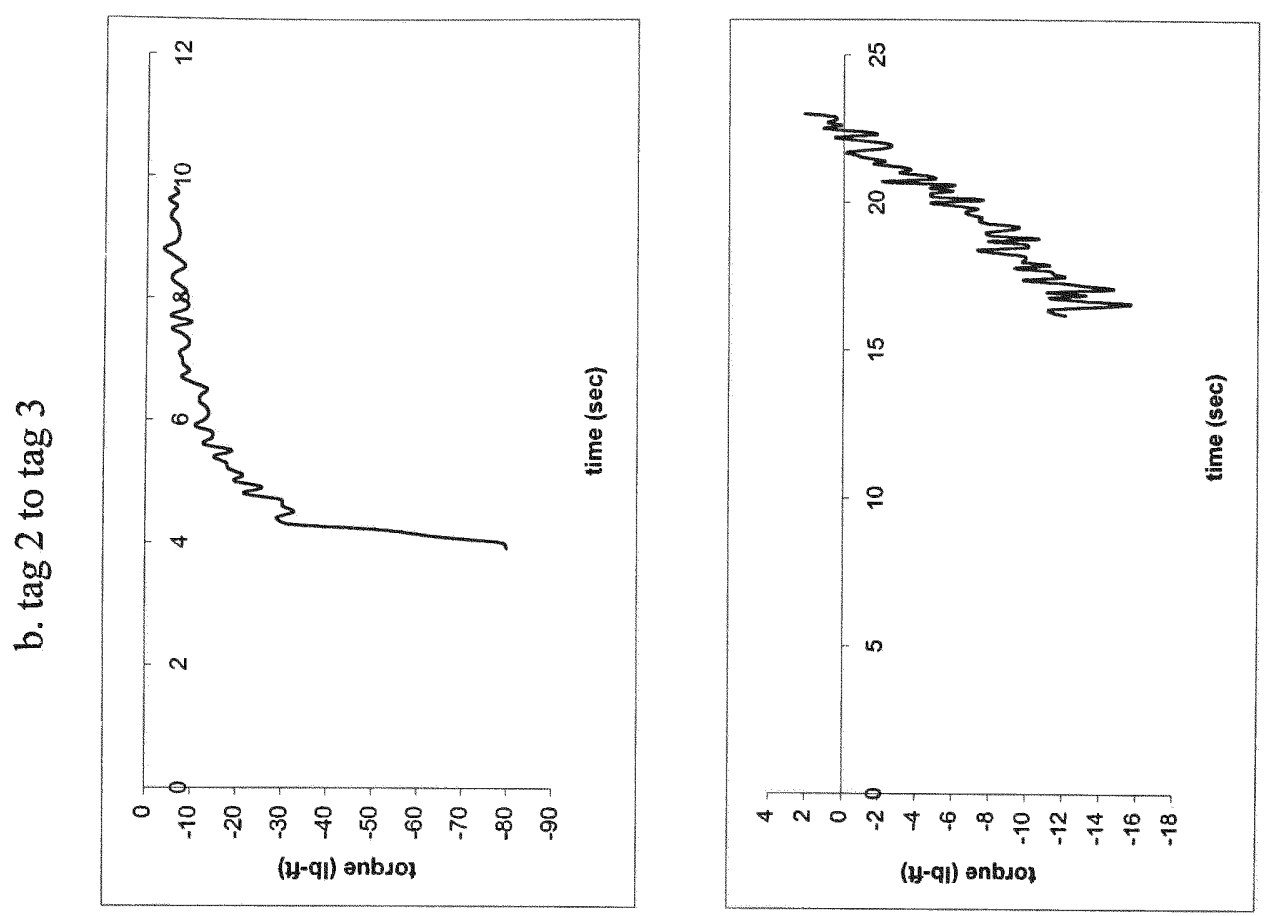

$n$
0
$\Xi$
0
0
0
00
$\Phi$
0

गं
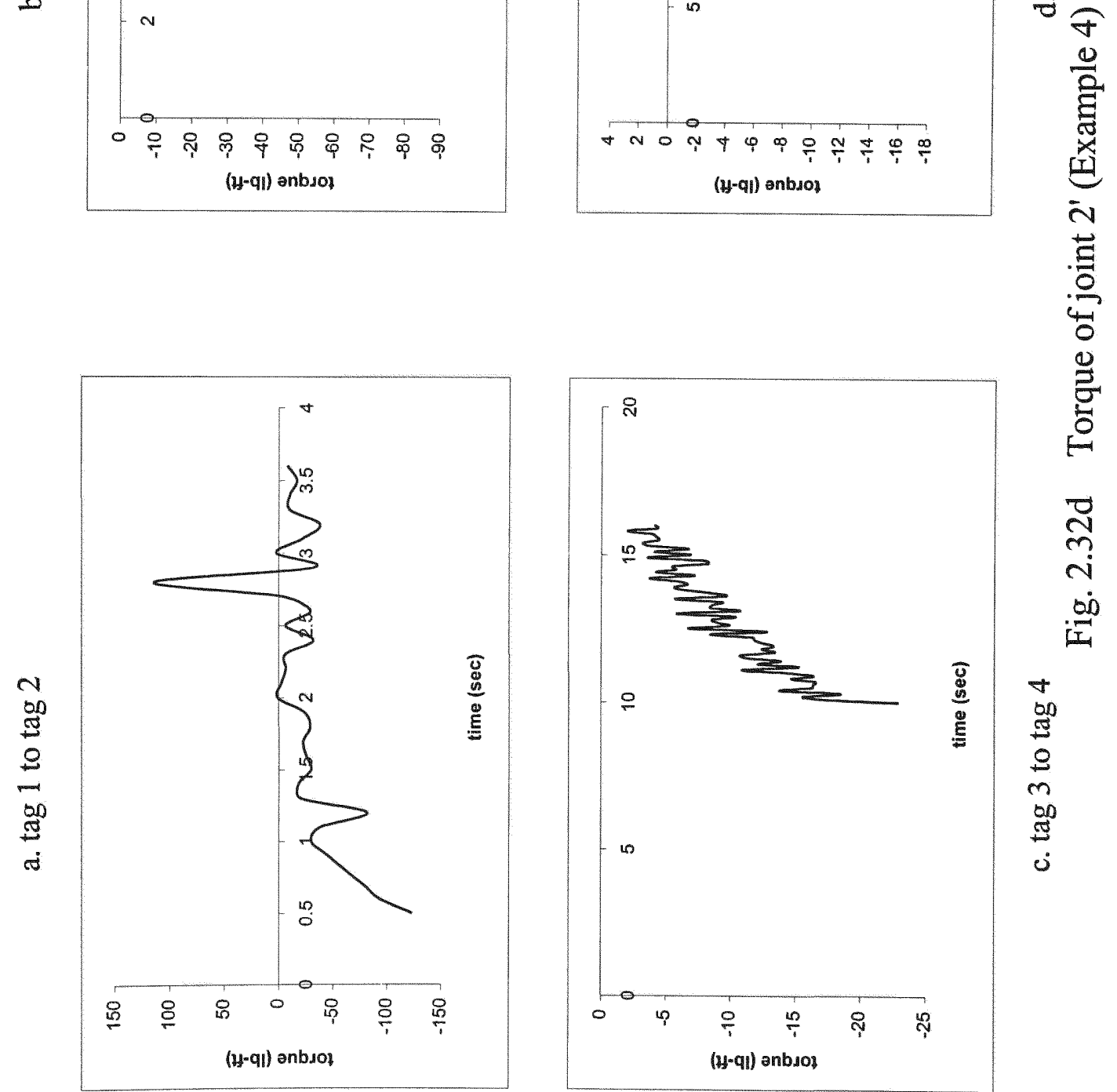


\section{CHAPTER 3}

\section{FINITE ELEMENT ANALYSIS OF THE FLEXIBLE LAMINATED HOSE}

\subsection{Introduction}

The first generation tank waste retrieval manipulator systems (TWRMS) are utilized to retrieve radioactive wastes from underground storage tanks. For the TWRMS currently deployed at ORNL (Oak Ridge National Laboratory), there are two major components operated simultaneously to accomplish the desired task. One of the components is the long-reach redundant manipulator- Modified Light Duty Utility Arm (MLDUA). Another critical component is the waste dislodging and conveyance (WD\&C) system. The focus in this chapter is to develop a finite element model of the flexible laminated hose, which is one of the critical parts in the WD\&C system, to investigate the deformation of the hose, stress and strain distribution on the hose and vibration of the manipulator resulted from the hose.

The flexible laminated hose utilized in WD\&C system is AQP 2661-32 elastomer tube Fig. 3.1. The suction hose is a bent elastic pipe with six degrees of freedom: translation in $\mathrm{X}, \mathrm{Y}, \mathrm{Z}$ directions and rotation about $\mathrm{X}, \mathrm{Y}, \mathrm{Z}$ axes. The tube is fixed at one end and subjected to different loading conditions at the other end, and liquid (mixture of water and waste materials) with very high viscosity is sucked through the hose using a vacuum pressure from the lower part (inlet) of the hose to the upper part (outlet) of the hose. The outer diameter of the hose is $2.55^{\prime \prime}$ and inner diameter of the hose is $2.00^{\prime \prime}$. The hose has a radius of curvature of $81.082^{\prime \prime}$. Both 7.5 feet and 10.5 feet hoses are used for the 25 feet and 50 feet tanks, respectively. The vacuum pressure in the hose is around 70 


\section{Wire inserted suction SAE 100R4

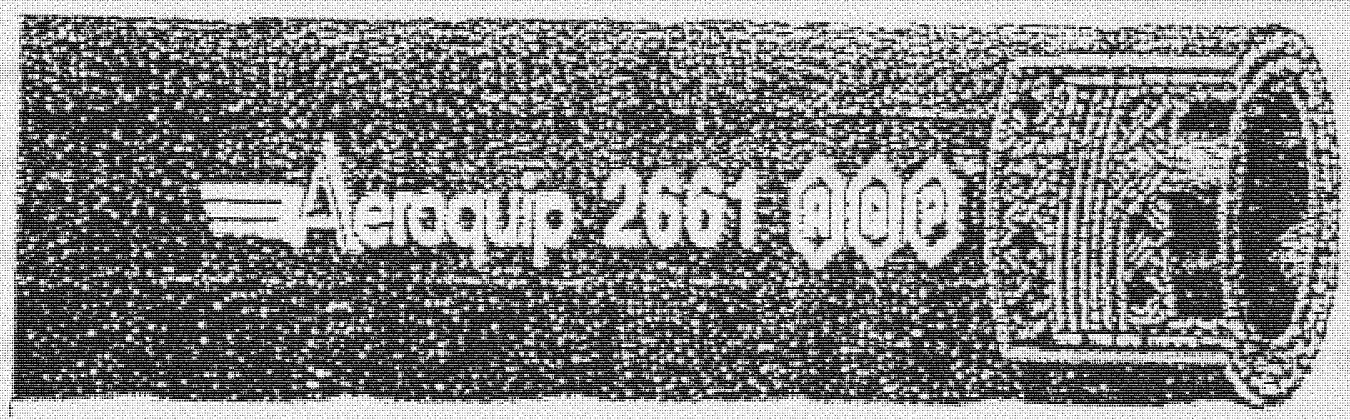

Fig. 3.1 The flexible laminated hose used in the WD\&C system 
psi. The structural behaviors of this elastomer tube are analyzed subjected to different loading conditions and ANSYS 52 finite element package is used.

\subsection{Properties of the Hose}

The hose is an elastomer tube with reinforcement consisting of a helical wire between two textile braids. The properties of this elastomer tube with reinforcement are not specified in any handbook. Hence, calculation of material properties of the hose is need.

Based on the known properties of elastomer and helical wire, the properties of the hose can be estimated. The Young's Modulus (E) of the hose is estimated as $5 E+5$ psi from the known material properties of polyester elastomer $(E=3 E+4$ psi $)$ and steel $(E=3 E+7 \mathrm{psi})$. Two layers of elastomer and one layer of helical steel wire are considered. In this case, Young's modulus of the elastomer tube is estimated as the average of 2 layers of polyester elastomer and one layer of steel. Similarly, poison's ratio $(0<\gamma<1 / 2)$

of the hose is estimated as 0.23 . Density $(\rho)$ of the tube is calculated as $1.84 \mathrm{E}-3 \mathrm{lbm} / \mathrm{in}^{3}$ from the known weight $(1.4 \mathrm{lb} / \mathrm{ft})$ and volume per foot $\left(1.365 \mathrm{E}-2 \mathrm{ft}^{3}\right)$.

\subsection{Structural Analysis of the Hose}

The purpose of the structural analysis of the hose is to investigate the deformation, stress distribution, and strain distribution on the entire laminated elastic hose. The procedures adopted to complete the analysis are 1). construction of the hose and mesh generation, 2). application of boundary conditions and loads on the system, and 3). FEA of deformation, stress and strain distributions of the hose. 


\subsubsection{Construction of the hose and mesh generation}

The construction of the hose and mesh generation is accomplished simultaneously. To generate the mesh, mixed node elements are used; that is, combination of the 2-D four-node plane 42 and the 3-D eight-node solid 45 elements are consodered. The plane 42 elements are used for the cross section of the hose, and the solid 45 elements are used for the entire section.

a). Plane 42 elements

The plane 42 elements are useful for modeling 2-D surfaces of a solid structure as shown in Fig. 3.2. One plane 42 element is defined by four nodes with two degrees of freedom at each node: translations in $\mathrm{x}$ and $\mathrm{y}$ directions. The element has plasticity, creep, swelling, stress, stiffening, large deflection and large strain capabilities.

b). Solid 45 elements

The solid 45 elements are used for defining 3-D elements of a solid structure, Fig. 3.3. One single solid 45 element consists of eight nodes with six degrees of freedom at each node: translations in $\mathrm{X}, \mathrm{Y}$ and $\mathrm{Z}$ directions and rotation about $\mathrm{X}, \mathrm{Y}$ and $\mathrm{Z}$ axes. The element has plasticity, creep, and swelling, stress stiffening, large deflection and large strain capabilities.

First, cross section of the hose is constructed using the plane 42 elements according to the known dimensions. The outer diameter of the hose is $2.55^{\prime \prime}$ and inner diameter is $2.00^{\prime \prime}$. Next, the 2-D plane 42 brick node elements are generated in ANSYS as shown in Fig. 3.4. Cross-section of the hose is extruded along the centerline of the hose with a radius of curvature of $81.082 "$. While extruding along the centerline, 20 

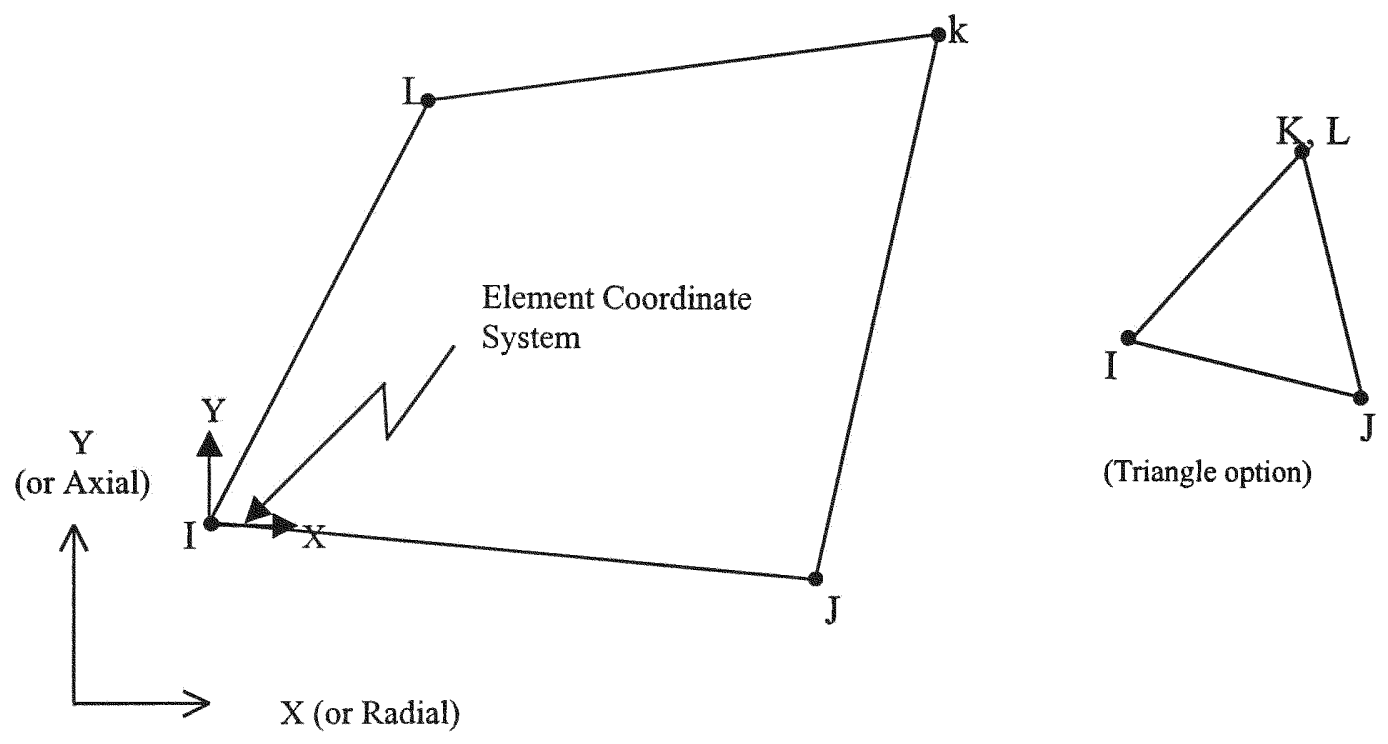

Fig. 3.2 A Plane 42 element defined in ANSYS 

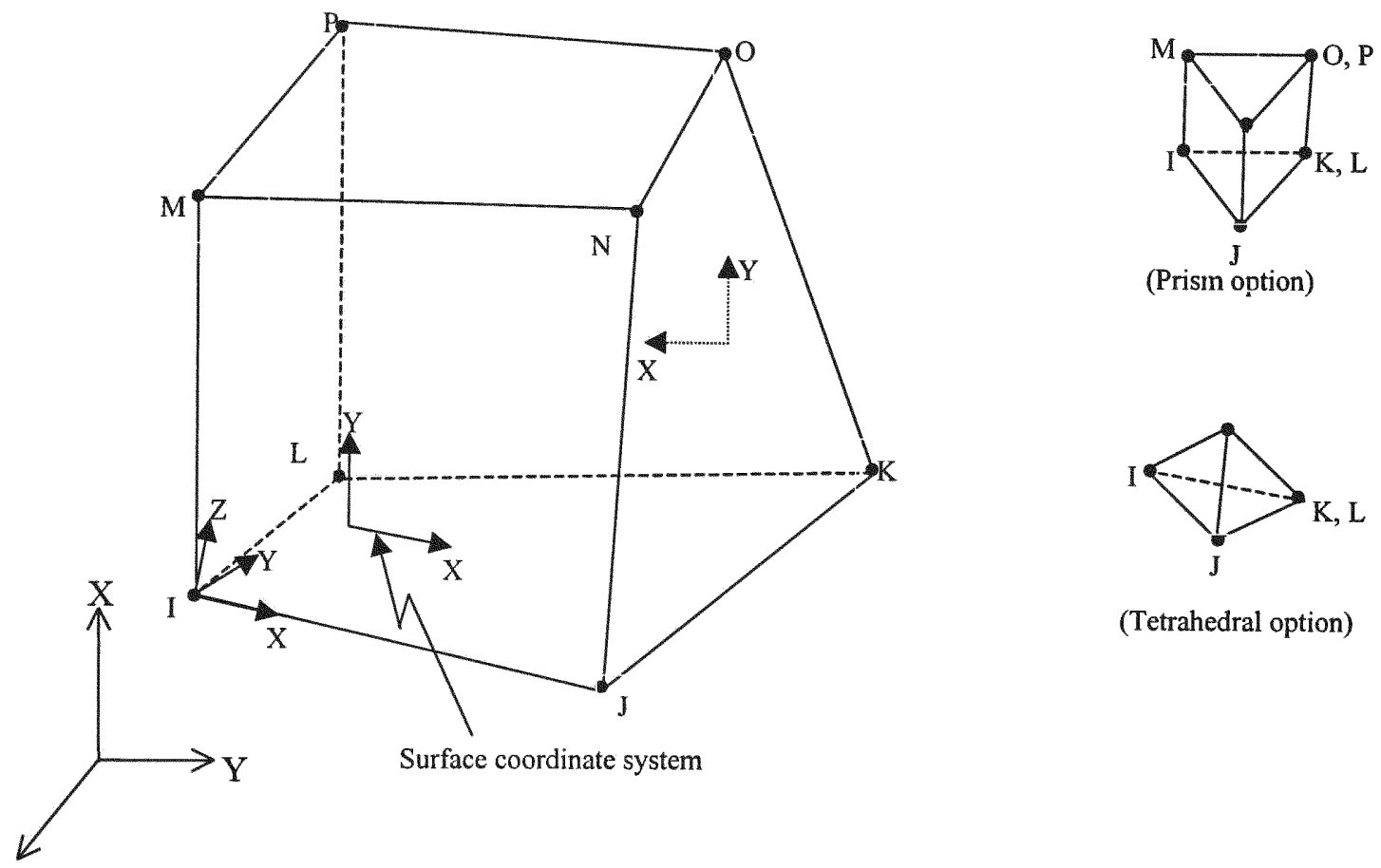

(Tetrahedral option)

Fig. 3.3 A solid 45 element defined in ANSYS 


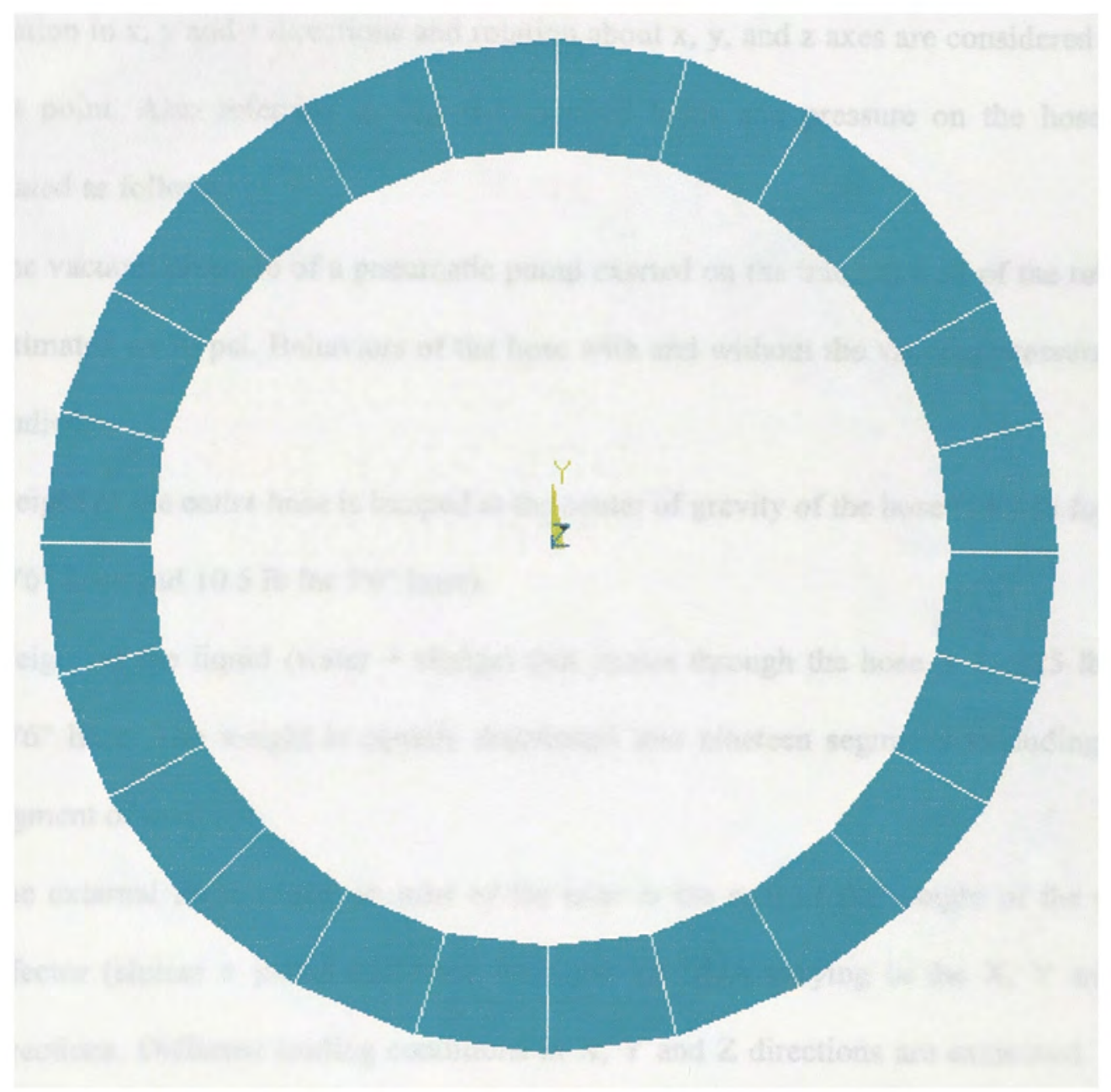

Fig. 3.4 Cross- sectional mesh 
elements of 3-D eight-node solid 45 elements are defined, and a 3-D mesh is depicted in Fig. 3.5.

\subsubsection{Boundary conditions and applied loads}

The flexible laminated hose is relatively fixed at its outlet (upper part). Hence its translation in $\mathrm{x}, \mathrm{y}$ and $\mathrm{z}$ directions and rotation about $\mathrm{x}, \mathrm{y}$, and $\mathrm{z}$ axes are considered zero at this point. Also referring to Fig. 3.5, applied loads and pressure on the hose are calculated as follows:

1. The vacuum pressure of a pneumatic pump exerted on the internal wall of the tube is estimated as $70 \mathrm{psi}$. Behaviors of the hose with and without the vacuum pressure are studied.

2. Weight of the entire hose is lumped at the center of gravity of the hose $(14.7 \mathrm{lb}$ for the $10^{\prime} 6^{\prime \prime}$ hose and $10.5 \mathrm{lb}$ for $7^{\prime} 6^{\prime \prime}$ hose).

3. Weight of the liquid (water + sludge) that passes through the hose is $21.225 \mathrm{lb}$ for $10^{\prime} 6 "$ hose. The weight is equally distributed into nineteen segments excluding the segment of inlet.

4. The external force acting on inlet of the hose is the sum of the weight of the endeffector (sluicer + parts) and force from the MLDUA varying in the $\mathrm{X}, \mathrm{Y}$ and $\mathrm{Z}$ directions. Different loading conditions in $\mathrm{X}, \mathrm{Y}$ and $\mathrm{Z}$ directions are examined. The ranges of $F_{x}, F_{y}$, and $F_{z}$ are: $0 \leq F_{x} \leq 60$ lbs $\left(\Delta F_{x}=20\right),-90 \leq F_{y} \leq 90$ lbs $\left(\Delta F_{y}=45\right)$, and $-40 \leq \mathrm{F}_{\mathrm{z}} \leq 40 \mathrm{lbs}\left(\Delta \mathrm{F}_{\mathrm{z}}=20\right)$. 


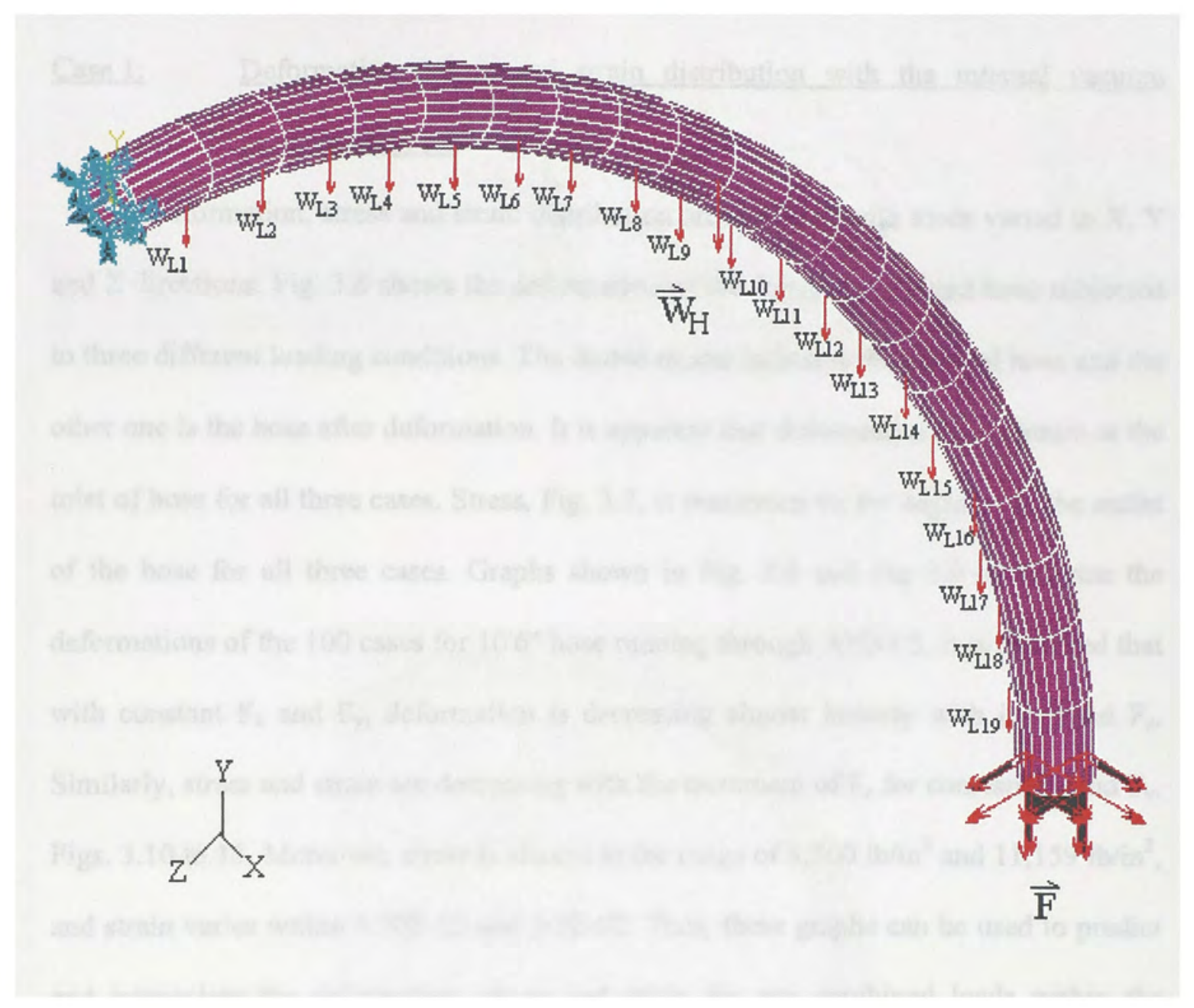

Fig. 3.5 Applied boundary conditions on the 3-D mesh 


\subsection{Finite Element Analysis}

The prescribed loading conditions are analyzed in 100 different cases by varying the values of loads in the specified range with and without the internal vacuum pressure of $70 \mathrm{psi}$.

Case 1: Deformation, stress and strain distribution with the internal vacuum pressure of $70 \mathrm{psi}$

Deformation, stress and strain distribution are analyzed with loads varied in $\mathrm{X}, \mathrm{Y}$ and $\mathrm{Z}$ directions. Fig. 3.6 shows the deformation of the flexible laminated hose subjected to three different loading conditions. The dotted model indicates the original hose and the other one is the hose after deformation. It is apparent that deformation is maximum at the inlet of hose for all three cases. Stress, Fig. 3.7, is maximum on the segment of the outlet of the hose for all three cases. Graphs shown in Fig. 3.8 and Fig 3.9 summarize the deformations of the 100 cases for $10^{\prime} 6^{\prime \prime}$ hose running through ANSYS. It is observed that with constant $F_{x}$ and $F_{y}$, deformation is decreasing almost linearly with increased $F_{z}$. Similarly, stress and strain are decreasing with the increment of $F_{z}$ for constant $F_{x}$ and $F_{y}$, Figs. 3.10 to 13 . Moreover, stress is always in the range of $8,500 \mathrm{lb} / \mathrm{in}^{2}$ and $11,159 \mathrm{lb} / \mathrm{in}^{2}$, and strain varies within $1.70 \mathrm{E}-02$ and $2.5 \mathrm{E}-02$. Thus, these graphs can be used to predict and interpolate the deformation, stress and strain for any combined loads within the elastic limits. 


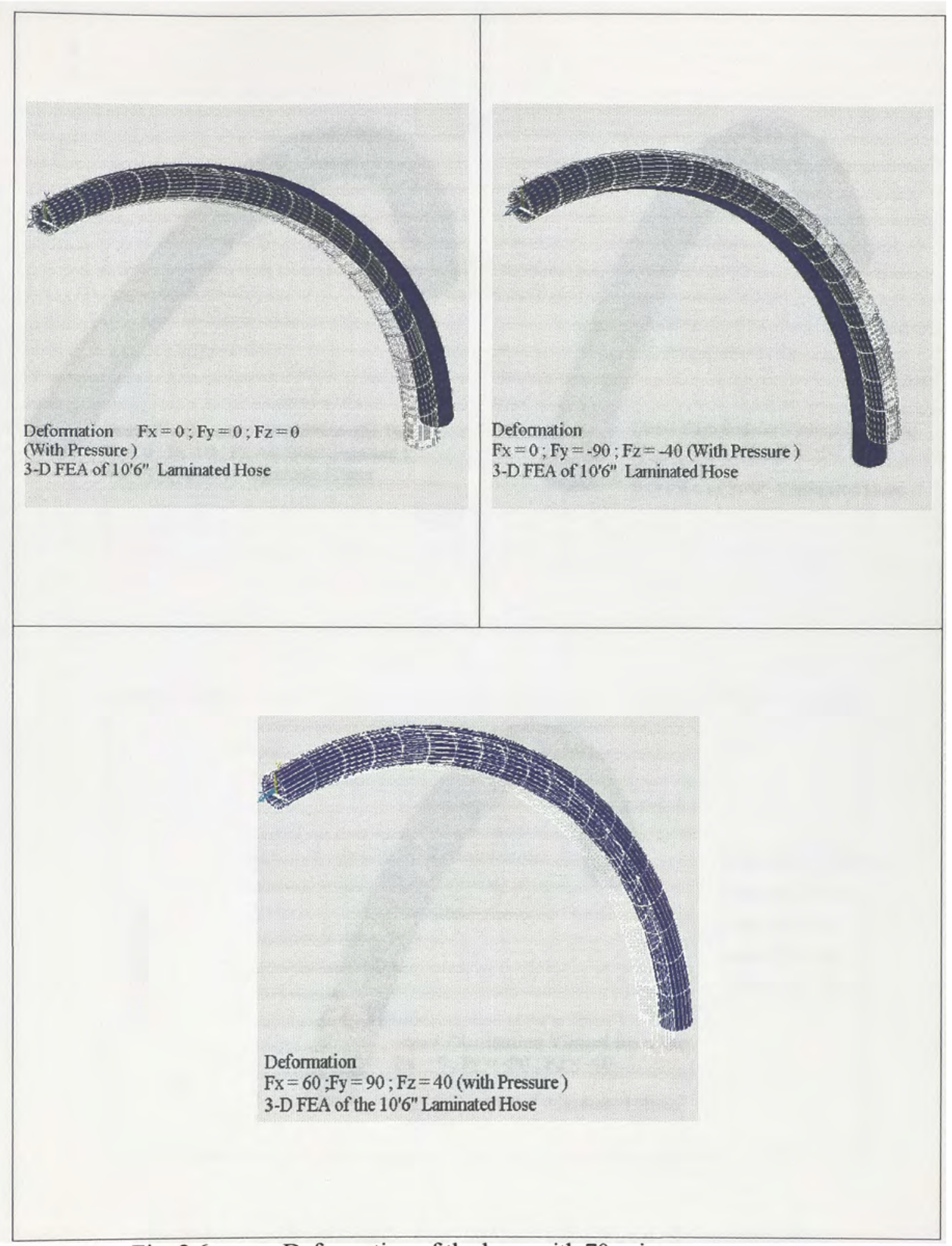

Fig. 3.6 Deformation of the hose with 70 psi vacuum pressure 


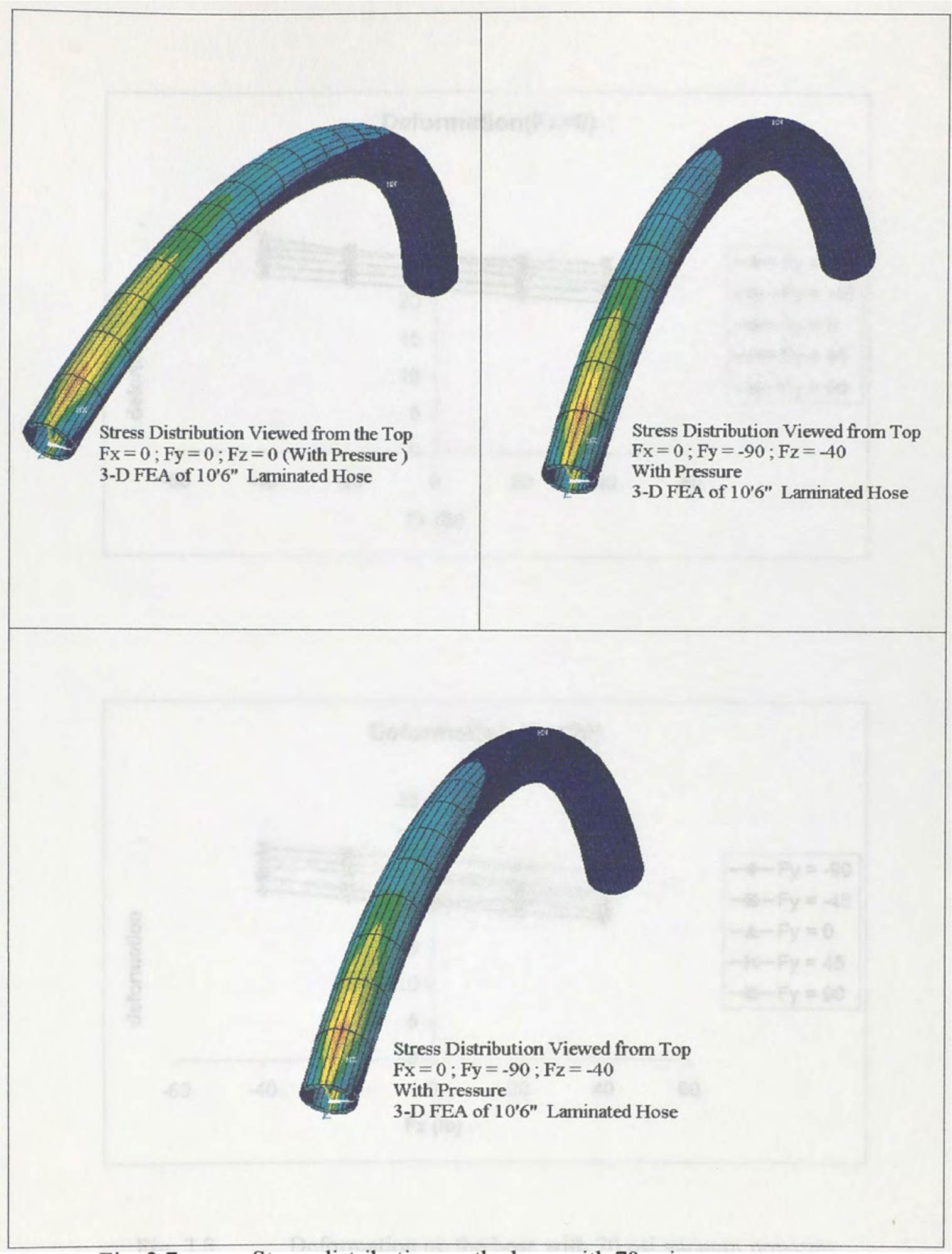

Fig. 3.7 Stress distribution on the hose with $70 \mathrm{psi}$ vacuum pressure 

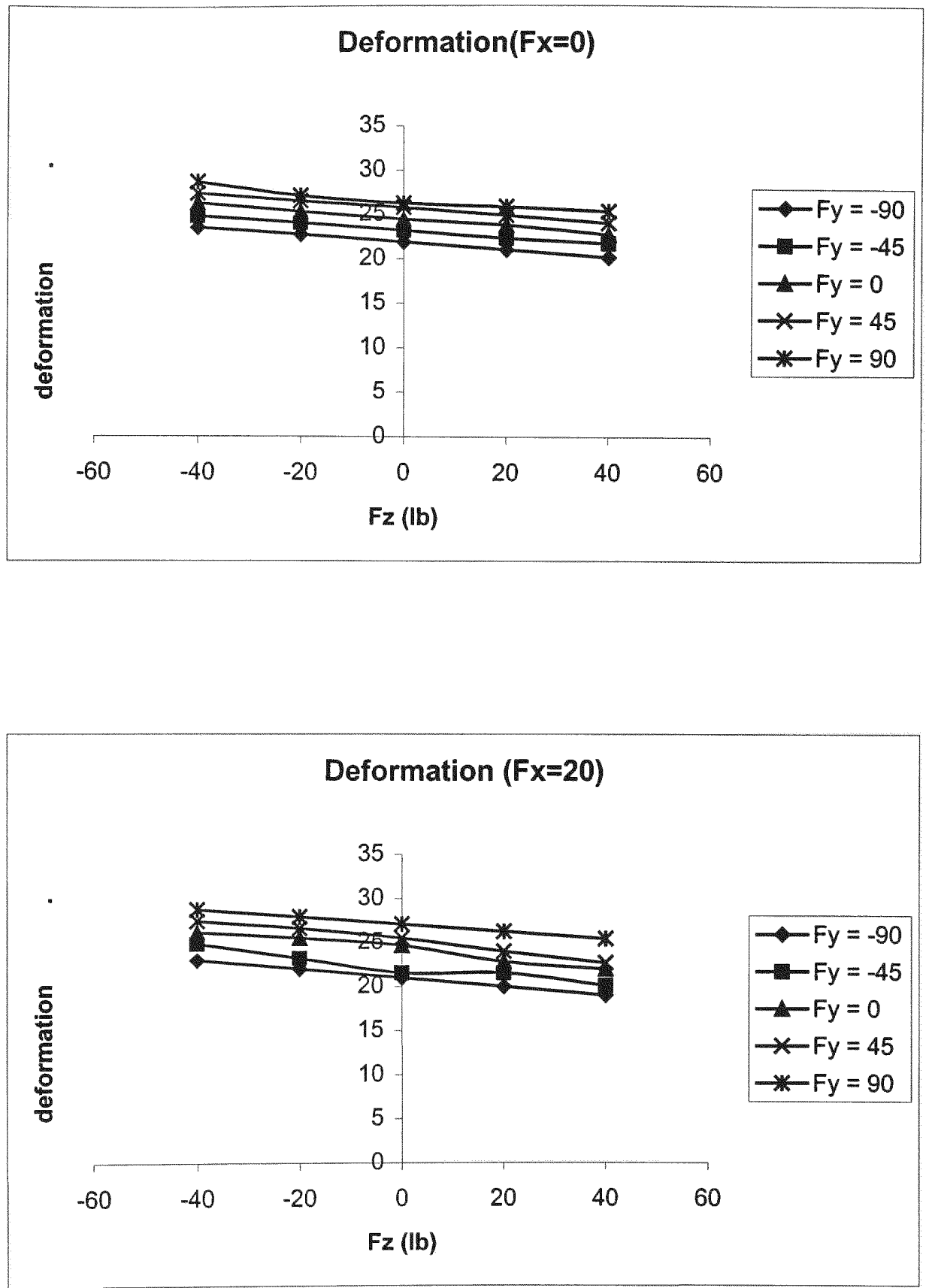

Fig. 3.8 Deformation on the hose with 70 psi vacuum pressure 

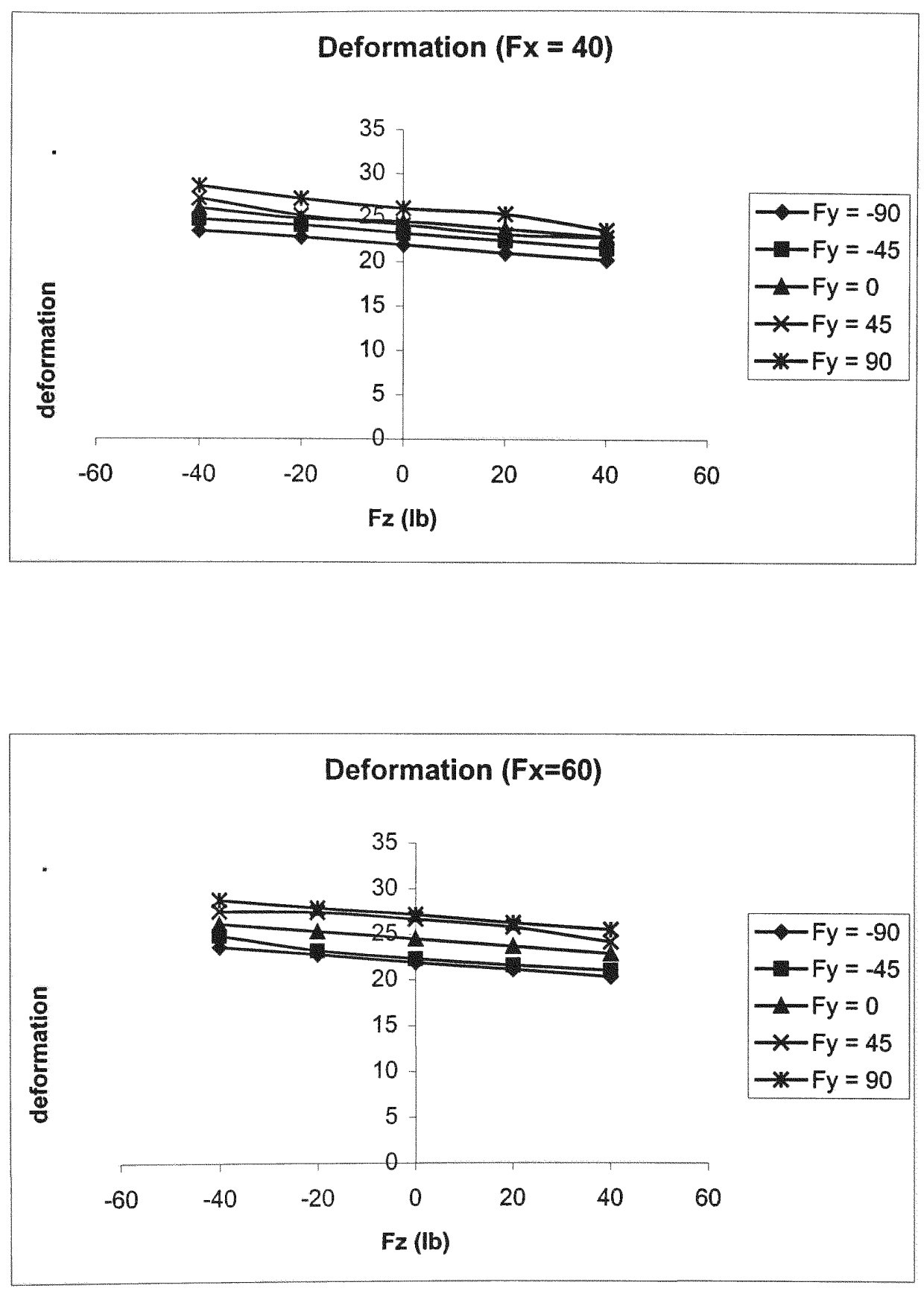

Fig. 3.9 Deformation of the hose with 70 psi vacuum pressure 

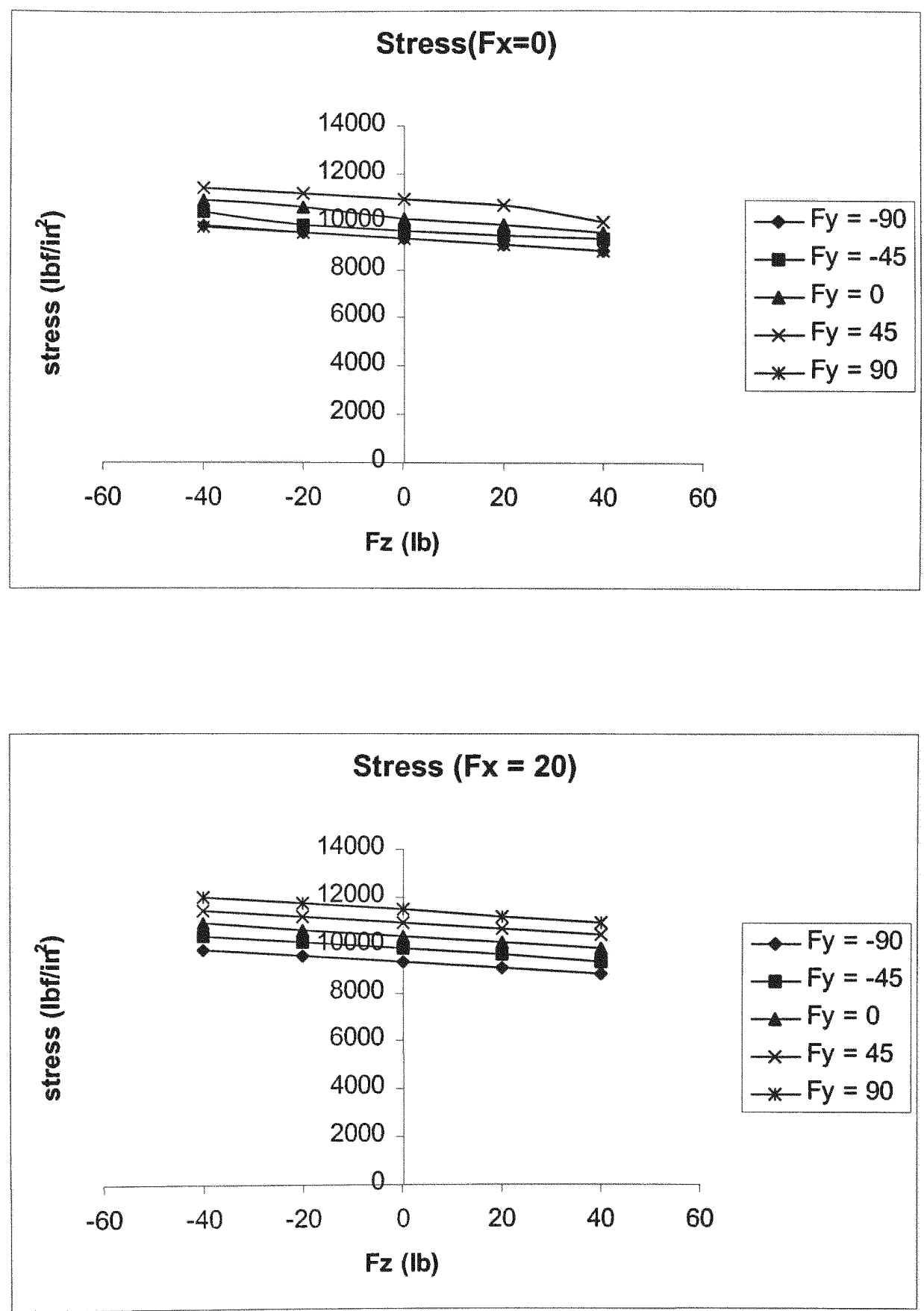

Fig. 3.10 Stress distribution on the hose with 70 psi vacuum pressure 

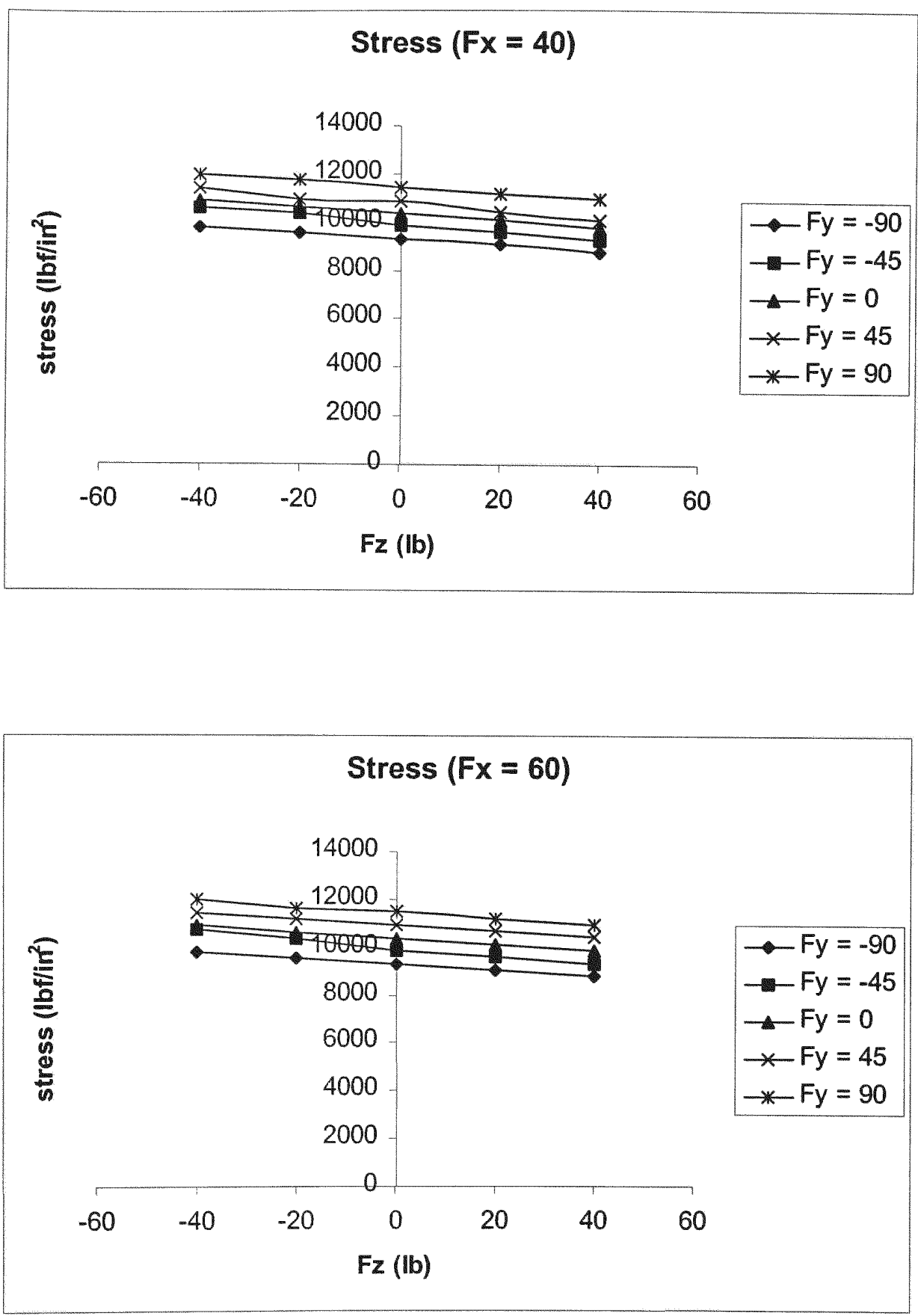

Fig. 3.11 Stress distribution on the hose with 70 psi vacuum pressure 

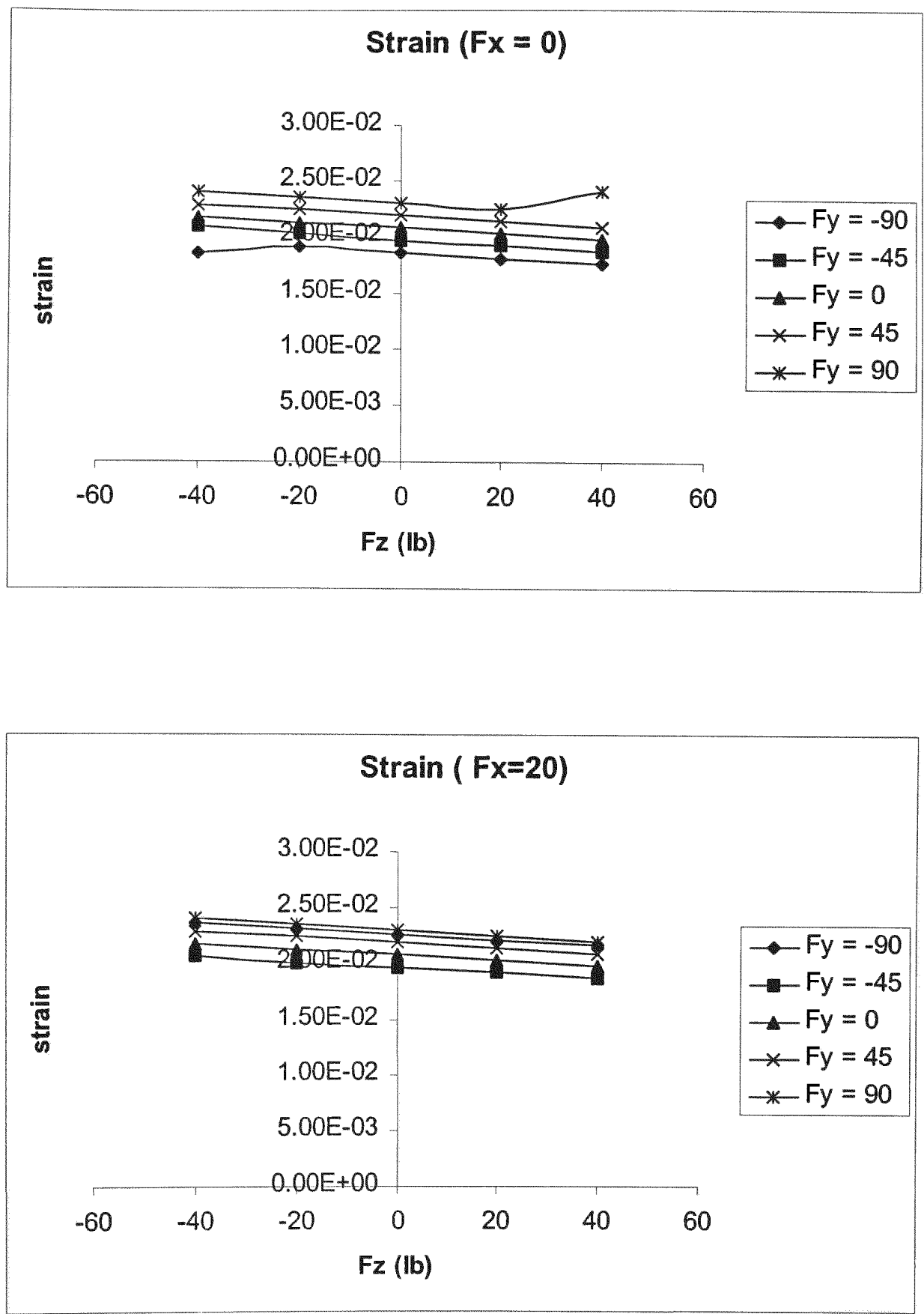

Fig. 3.12 Strain distribution on the hose with 70 psi vacuum pressure 

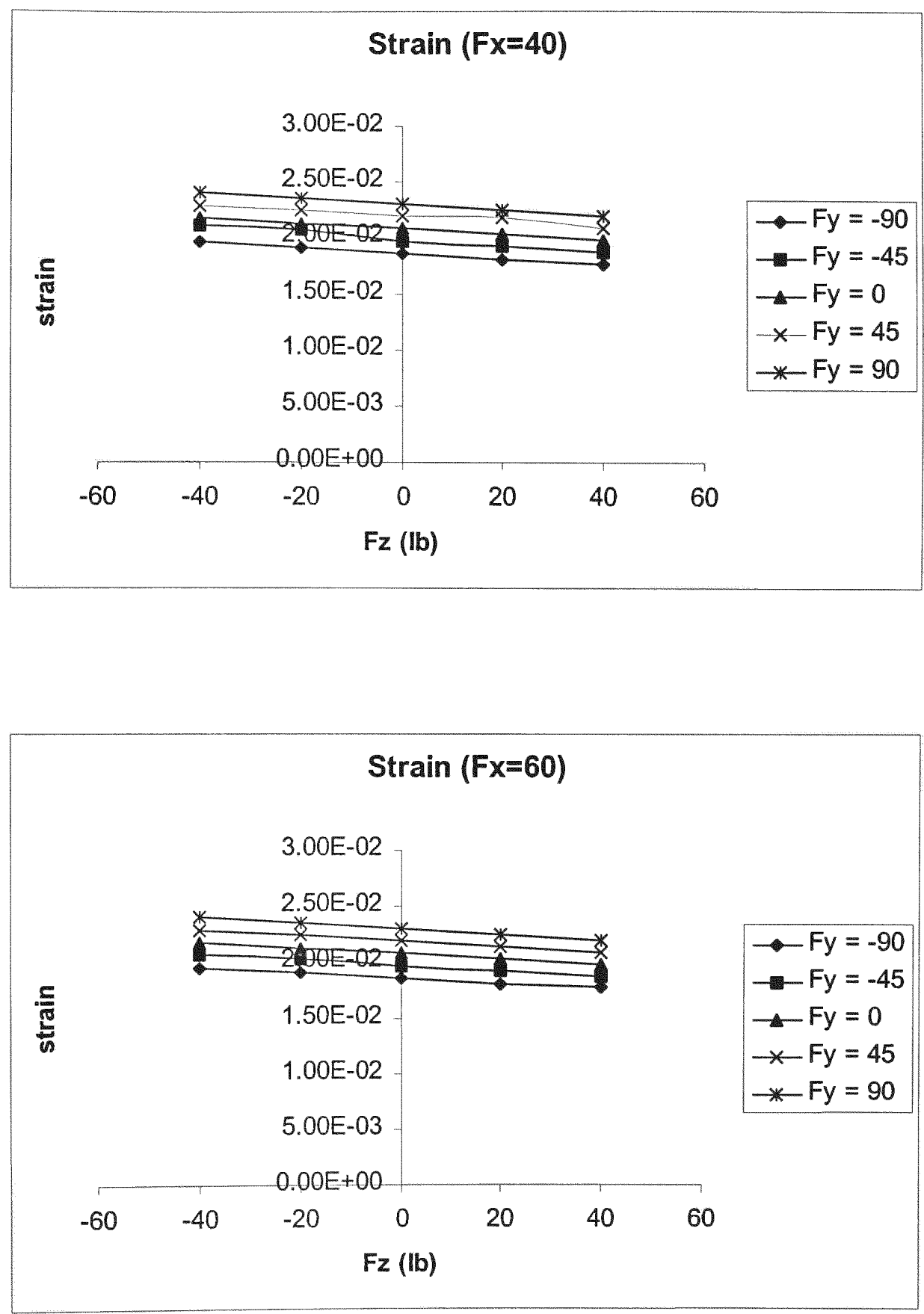

Fig. 3.13 Strain distribution on the hose with 70 psi vacuum pressure 
Case 2: Deformation, stress and strain distribution without the internal vacuum pressure of $70 \mathrm{psi}$

In this case, deformation, stress and strain distribution are solved, without the vacuum pressure of $70 \mathrm{psi}$, for 100 cases by varying the loads in $\mathrm{X}, \mathrm{Y}$ and $\mathrm{Z}$ directions at the inlet of the hose. Deformation, Fig. 3.14, is maximum at the inlet where the force is applied to it. It is decreasing from inlet of the hose to the outlet. Stress is maximum at the segment of the outlet similar to that in case 1 as depicted in Fig. 3.15. Curves of deformation, stress and strain for 100 different cases analyzed in ANSYS are shown in Figs. 3.16 to 22. Deformation, stress and strain are increasing with the increment of $F_{z}, F_{x}$ and $F_{y}$ are constants. Stress varies within the range of $400 \mathrm{lb} / \mathrm{in}^{2}$ and $4,000 \mathrm{lb} / \mathrm{in}^{2}$, and strain varies within $2.0 \mathrm{E}-4$ and $4.5 \mathrm{E}-3$. In comparison, deformation, stress and strain are much higher when vacuum pressure is applied to the flexible laminated hose. 


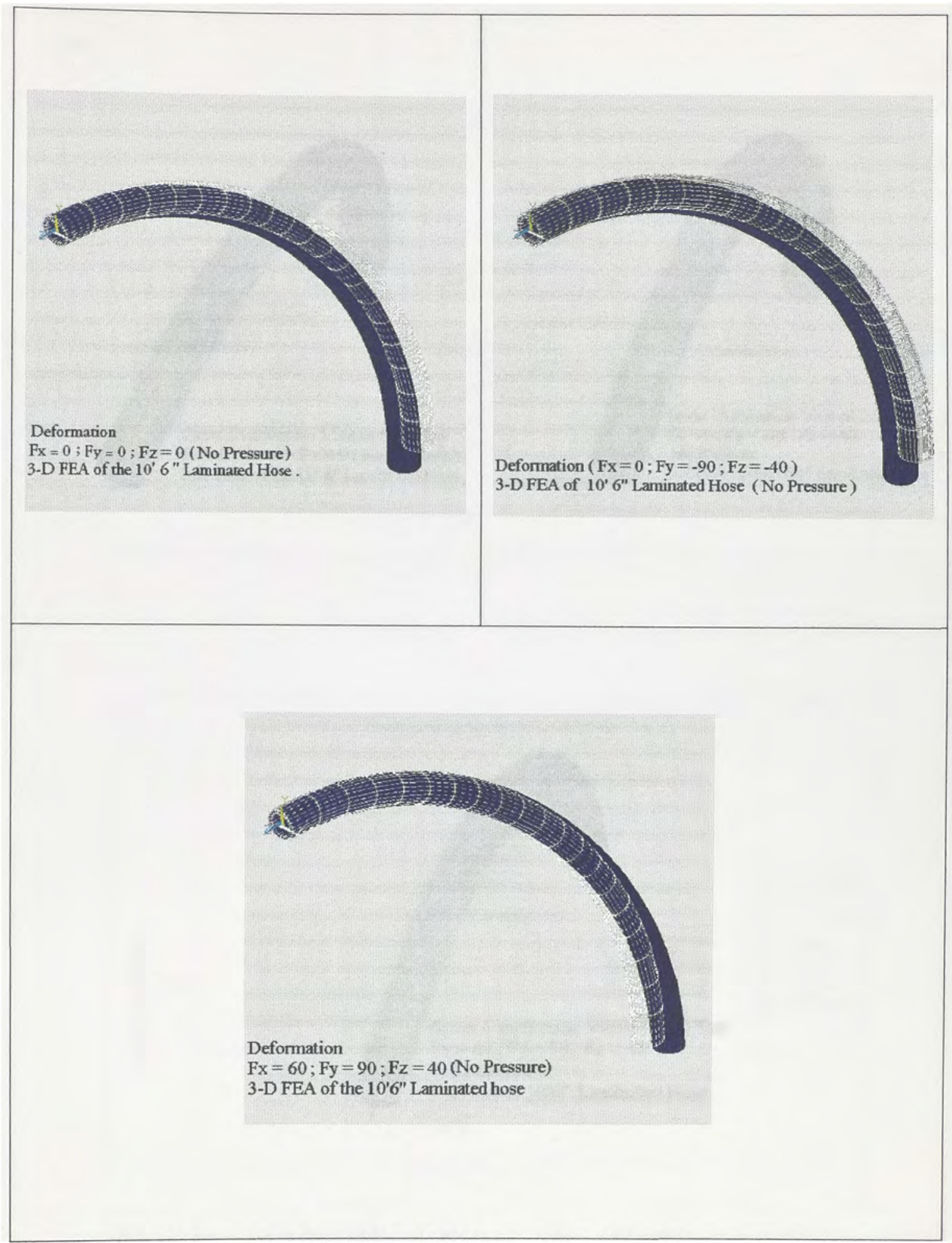

Fig. 3.14 Deformation of the hose without 70 psi vacuum pressure 


$$
\begin{gathered}
C r \\
C
\end{gathered}
$$



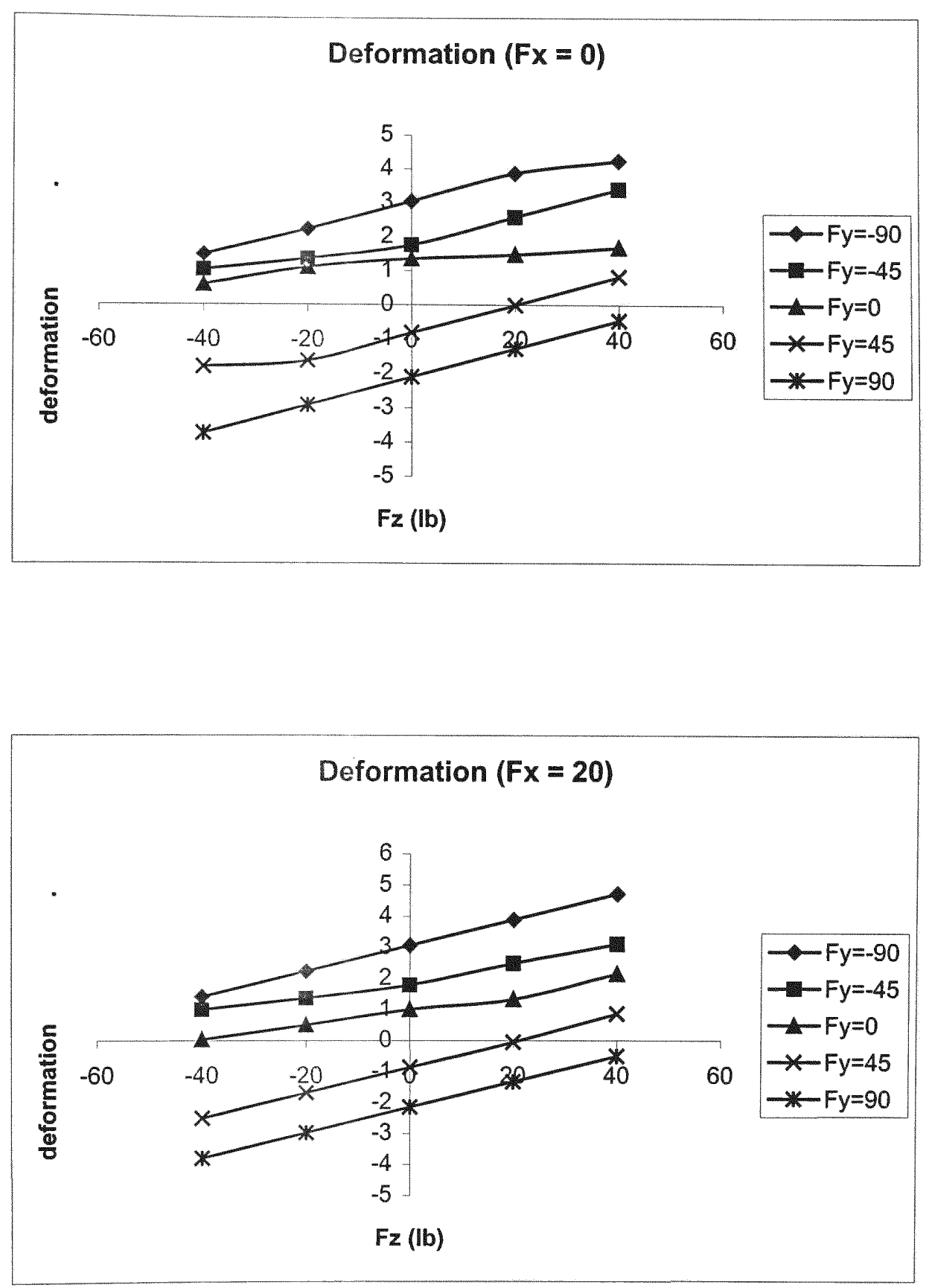

Fig. 3.16 Deformation of the hose without 70 psi vacuum pressure 

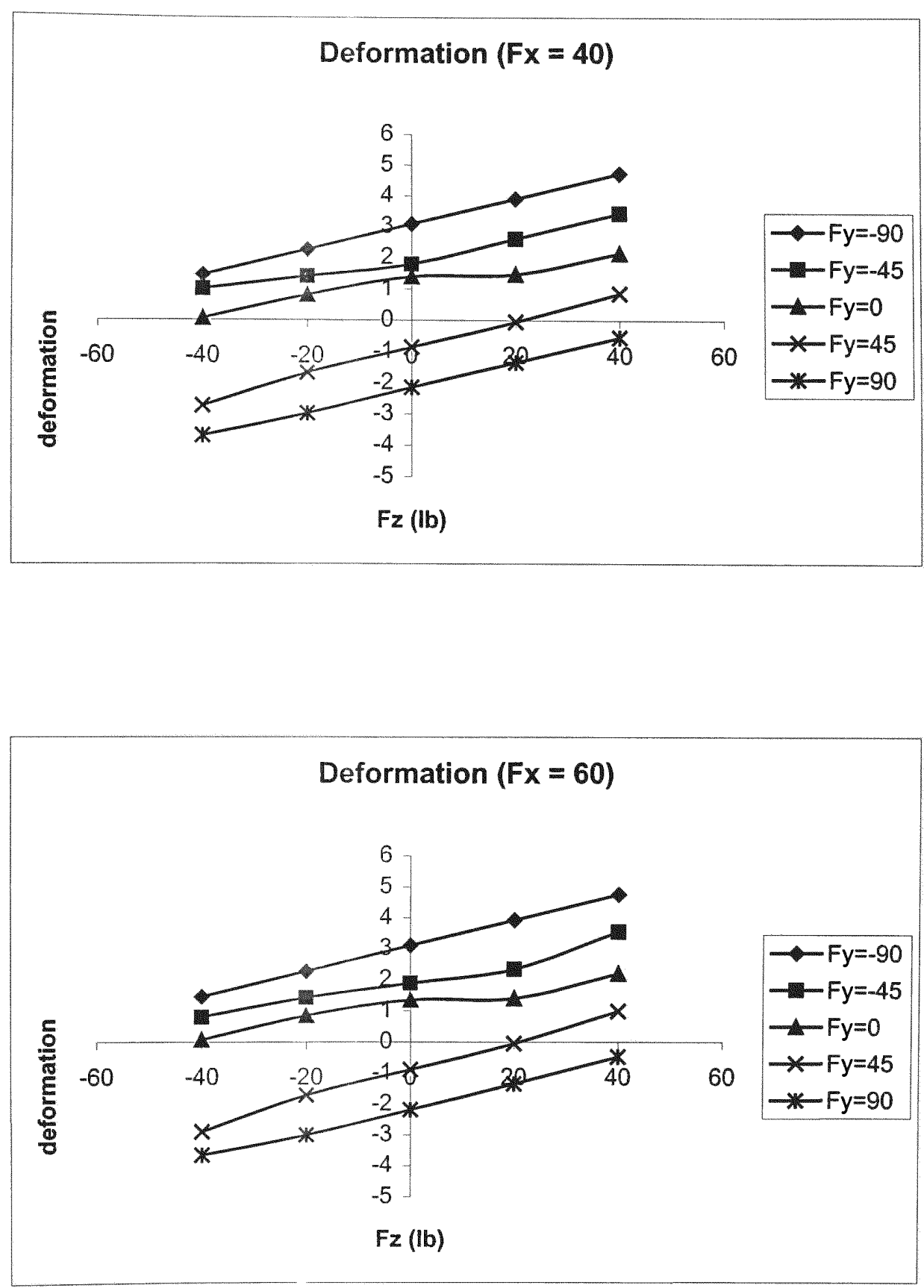

Fig. 3.17 Deformation of the hose without 70 psi vacuum pressure 

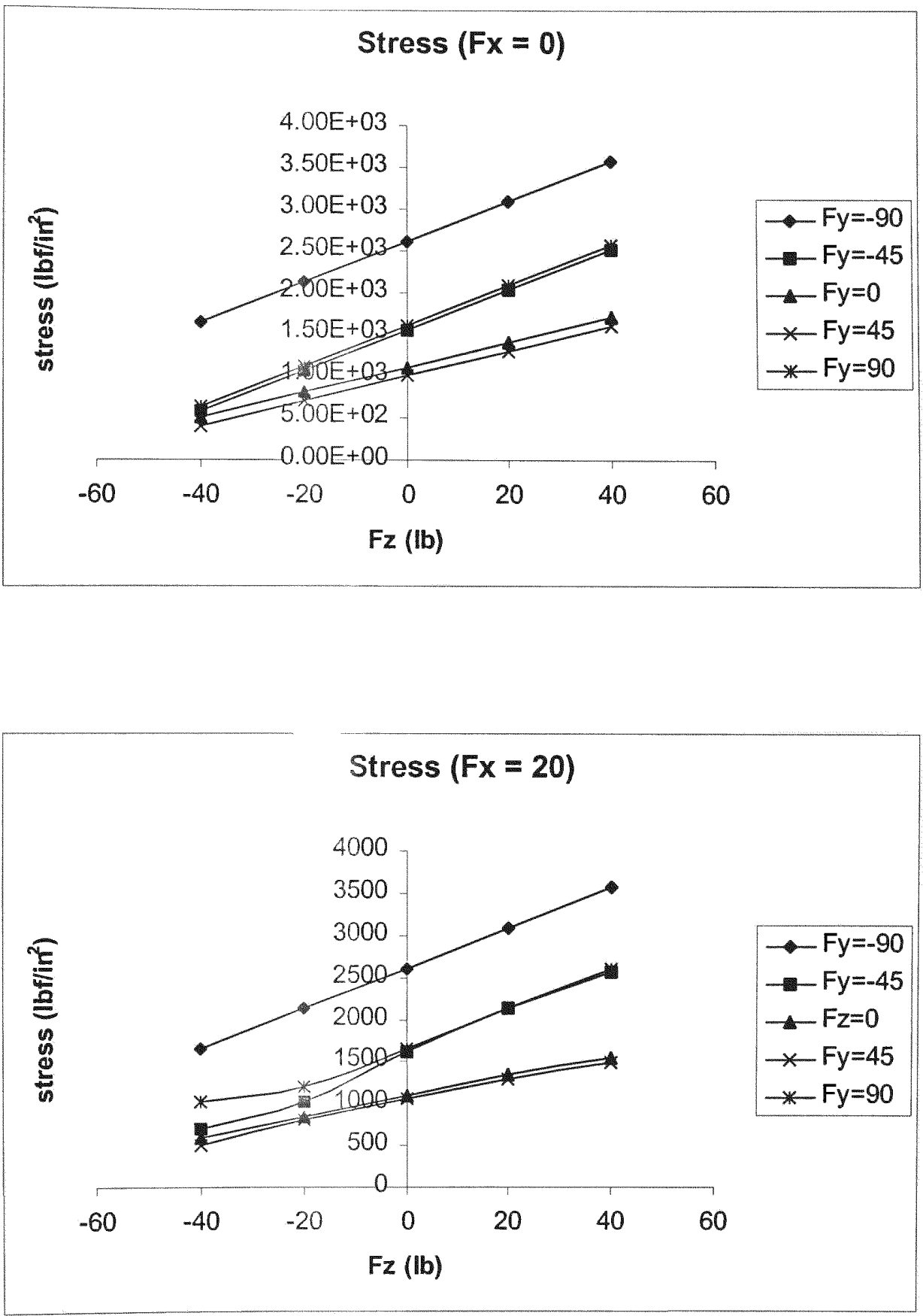

Fig. 3.18 Stress distribution on the hose without 70 psi vacuum pressure 

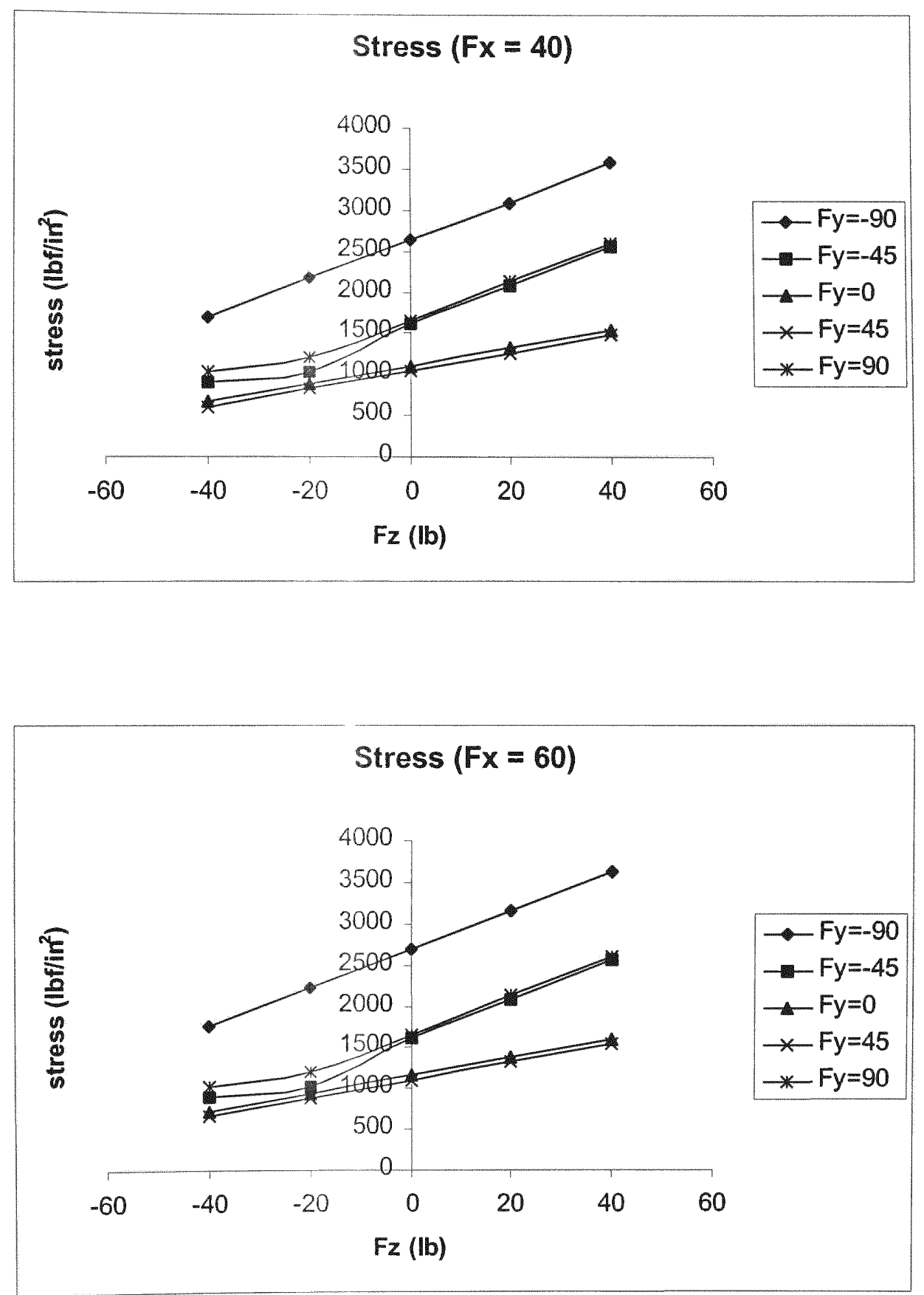

Fig. 3.19 Stress distribution on the hose without 70 psi vacuum pressure 

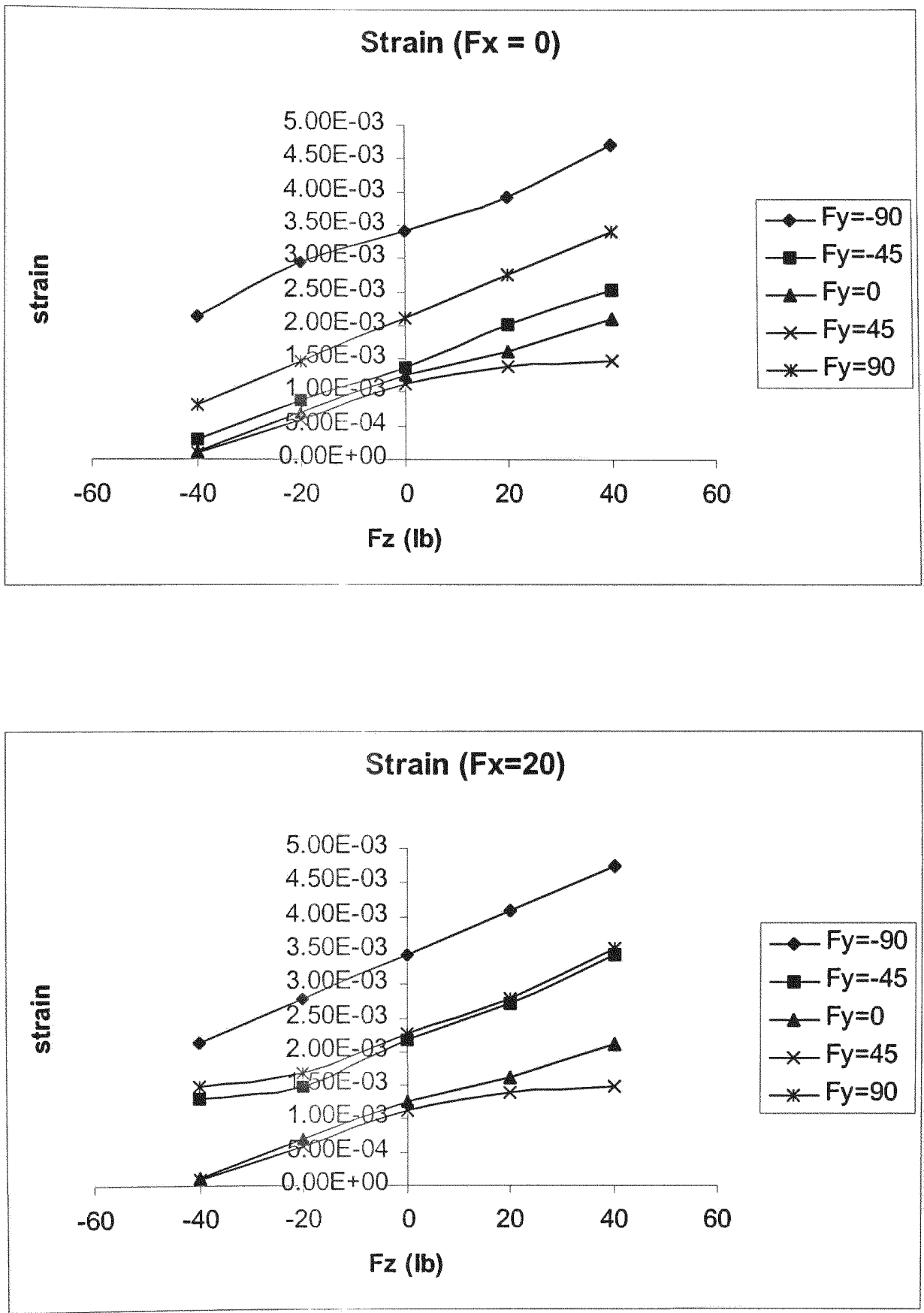

Fig. 3.20 Strain distribution on the hose without 70 psi vacuum pressure 

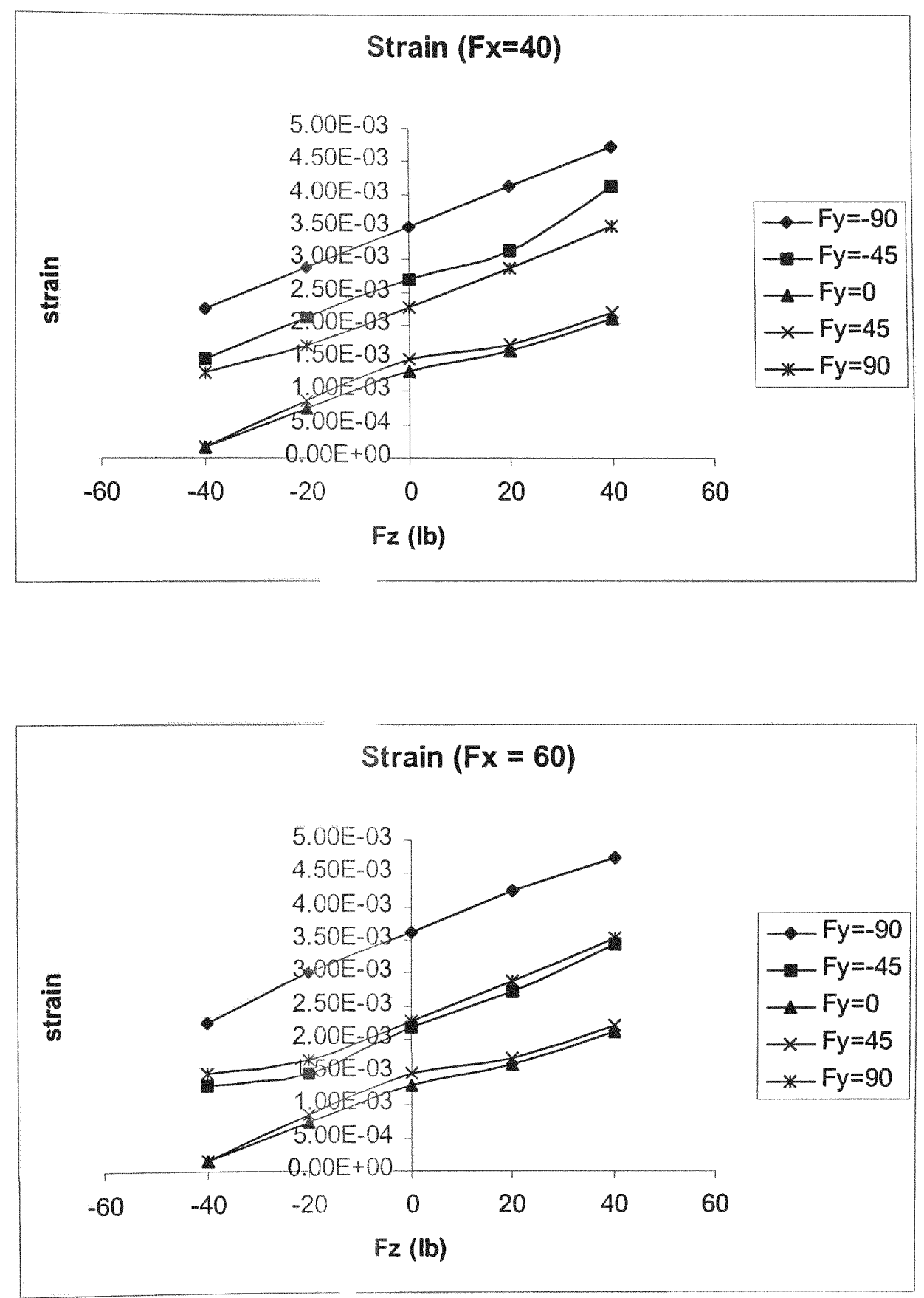

Fig. 3.21 Strain distribution on the hose without 70 psi vacuum pressure 


\section{CHAPTER 4}

\section{CONCLUSIONS AND PERSPECTIVE}

A kinamatic model based on the $4 \mathrm{X} 4$ homogeneous matrix $\mathrm{C}-\mathrm{B}$ notation for the Phase 3 RTD system was developed successfully. The Phase 3 RTD system is used to retrieve wastes from the underground storage tank with internal structural members. In addition, a dynamic model of the Phase 3 RTD system was developed. The dual-gripper design and inclusion of the VTM distinguish the Phase 3 RTD system from the other robotic systems. The special features provide greater dexterity and flexibility. Two out of the four possible operating configurations of the Phase 3 RTD system were focused, analyzed and simulated. In the thesis, two open-chain configurations have been analyzed and simulated successfully using Tele/IGRIP simulation package. Case study was done for two trajectories followed by the Phase 3 RTD system as an open-chain robotic system. The first open-chain configuration is gripper 1 grasp the structural member and gripper two is free to move; and the other configuration is with gripper 2 fixed to the structural member. Each joint angular position, velocity and acceleration and joint torque have been calculated and graphed. Investigation of the other two closed-chain configurations should be continued as future work. The first closed-chain configuration is that both grippers grasp two separate structural members and the orion slave arm is attached to link 3 to operate designated task. The second closed-chain configuration is that both grippers grasp the same structural member.

The graphs developed can be used by the operator to select the best operation configuration. To further analyze the collected data and to compare both cases for better operating configuration, the optimal (minimum/maximum) values of each joint are 
tabulated in Table 4.1. Although this table does not provide definite conclusions for the best configuration, it still can be a useful tool to select a feasible operating configuration under the consideration of different operating conditions. For example, to minimize the motion (displacement) of the gripper joint, case 1 (gripper 1 is fixed and gripper 2 is in motion) will be the feasible operation configuration. If smaller torque at the VTM is desired then case 2 (gripper 2 fixed and gripper 1 is in motion) will be a better selection. It is also observed from the Tele/IGRIP simulation of numerous operation conditions, case 1 is a better configuration if the RTD system is to clean the areas distal to gripper 1; that is, the fixed structural member. However, to clean the proximal areas that surround the fixed structural member, case 2 will be a better choice.

Finite Element Analysis (FEA) of the 10'6" flexible laminated hose used to convey the radioactive waste out of the underground storage tank has been completed as well. ANSYS 5.2 was used to analyze the laminated hose to learn about its deformation, stress and strain distributions with and without the internal vacuum pressure in the hose. Mixed node elements, i.e. the combination of plane 42 elements and solid 45 elements was used to better understand the behavior of the hose. Two sets of curves, each generated from a total of 100 cases, can be used to predict and interpolate the deformation, stress and strain for any combined loads within the elastic limits. Future work has to be done to study the behavior of the $7^{\prime} 6^{\prime \prime}$ flexible laminated hose. 
Table 4.1 Summary of optimal kinematic and dynamic data

\begin{tabular}{|c|c|c|c|}
\hline MOTION ANALYSIS & JOINT & CASE 1 & CASE 2 \\
\hline \multirow{7}{*}{$\begin{array}{c}\text { Displacement } \\
(d e g)\end{array}$} & 2 & $-8.07 / 57.07$ & $-125.19 / 0$ \\
\hline & 3 & $0 / 26.6$ & \\
\hline & 4 & & $-25.62 / 0$ \\
\hline & 5 & $-6.22 / 99.6$ & $-74.05 / 0$ \\
\hline & 6 & $0 / 59.08$ & $-73.13 / 3.03$ \\
\hline & 7 & $0 / 110.79$ & $-65.54 / 4.18$ \\
\hline & Gripper 2/1 & $0 / 110.79$ & $-125.19 / 0$ \\
\hline \multirow{7}{*}{$\begin{array}{l}\text { Velocity } \\
(\text { deg/sec })\end{array}$} & 2 & $-7.66 / 4.74$ & $-10.93 / 7.74$ \\
\hline & 3 & $0 / 2.13$ & \\
\hline & 4 & & $-0.96 / 0$ \\
\hline & 5 & $-9.87 / 8$ & $-8.61 / 2.96$ \\
\hline & 6 & $-5.65 / 11.17$ & $-9.7 / 9.37$ \\
\hline & 7 & $-14.44 / 12.69$ & $-11.37 / 2.99$ \\
\hline & Gripper 2/1 & $-14.44 / 12.69$ & $-10.93 / 7.74$ \\
\hline \multirow{7}{*}{$\begin{array}{c}\text { Acceleration } \\
\left(\operatorname{deg}^{\prime}\left(\sec ^{\wedge} 2\right)\right.\end{array}$} & 2 & $0 / 1.33$ & $0 / 2.83$ \\
\hline & 3 & $0 / 0.05$ & \\
\hline & 4 & & $0 / 0$ \\
\hline & 5 & $-0.33 / 2$ & $-1.33 / 1.98$ \\
\hline & 6 & $-0.7 / 0.85$ & $-3.53 / 2.43$ \\
\hline & 7 & $-3.44 / 0$ & $-3.38 / 2.51$ \\
\hline & Gripper 2/1 & $-3.44 / 0$ & $0 / 2.83$ \\
\hline \multirow{6}{*}{$\underset{(l b-f t)}{\mathbf{D} \mathbf{I N} \mathbf{A M I C S}}$} & 2 & $-7.049 / 13.882$ & $-1.589 / 0.439$ \\
\hline & 3 & $1,050.93 / 1,265.8$ & \\
\hline & 4 & & $-721.974 /-648.335$ \\
\hline & 5 & $0 / 7.053$ & $-4.3 /-0.11$ \\
\hline & 6 & $0 / 8.21$ & $-8.093 / 6.105$ \\
\hline & 7 & $-0.219 / 4.984$ & $-10.946 / 15.374$ \\
\hline
\end{tabular}




\section{REFERENCES}

1. Arthur J. Critchlow "Introduction to robotics" chapter 10 Macmillan Publishing Company1985

2. Micheal Erdmann "An exploration of Nonprehensile Two-Palm Manipulation"

The international journal of robotics research v 17 n May 1998. P 485-503

3. Tachung Yih "A new method for the Geomentric modelling of Lower pairs and its application to the kinematic spaces spatial robots " Journal of robotic systems Vol. 8, No. 4, August 1991

4. J Denavit, R S Hartenberg "A kinematic notation for lower-pair mechanisms based on matrices" ASME Trans.J. Appl.Mech. 1977. P 215-221

5. P N Sheth "A digital computer based simulation procedure for multiple degree of freedom mechanical system with geometric constrains" Ph.D. Dissertation, University of Wisconsin, Madison, WI,1972

6. P N Sheth "Improved iterative techniques for the $4 \times 4$ matrix method for kinematic analysis" MS thesis, University of Wisconsin, Madison, WI,1968

7. $\mathrm{R} \mathrm{C} \mathrm{Geortz,} \mathrm{"Manipulation} \mathrm{Used} \mathrm{for} \mathrm{handling} \mathrm{radioactive} \mathrm{materials"} \mathrm{Human}$ Factors in Technology", Chapter 27, edited by E.M. Bennett, McGraw-Hill, 1963

8. J Rosenberg, "A History of Numerical control 1949-1972: the Techanical Development, Transfer to Industry, and assimilation" U.S.C Information science Institute, marina Del Rey, California, Report No. ISI-RR72-3,1972

9. J J Uicker, Jr "Dynamic behavior of spatial linkages: Part 1-exact equations of motion; Part 2- small oscillation about equilibrium," Journal of engineering, ASME P 251-265, 1969

10. $\mathrm{D} \mathrm{E}$ Whitney "Resolved motion rate control of manipulators" IEEE transaction on man-machine systems MMS-10 1969. P 47053

11. J Baillieul "Kinematic programming alternatives for redundant manipulators" IEEE International conference on Robotics and Automation 1985. P 722-728

12. R L Anderson "Computing the feasible configuration of a 7-dof arm subject to joint limits" IEEE Transactions of Robotics and Automation, April 1993. P 187-195 
13. W A Wolovich and $\mathrm{H}$ Elliott "A computation Technique for Inverse Kinematics" proceedings of $23^{\text {rd }}$ IEEE conference on Decision and Control. 1984. P 1359-1363

14. Kovecses, J "Distributed parameter model for the dynamics of the flexible robots" Journal of robotic systems v 15 n 5 May 1998. P 281-298

15. Chen I-Ming, Yang Guillin "Automation generation of dynamics for modular robots with hybrid geometry" IEEE International conference on Robotics and Automation v 3 1997. P 2288-2293

16. Zhao Zhanfang, Shi Xiaolun "Dual-space approach for optimization of dynamic and kinematic performance of redundant manipulators" Journal of Beijing Institute of Technology v 6 n 2 June 1997. P 163-172

17. Pennock G.R., Mattson K.G "Analytical and graphical solution to the forward position probrlm of robots manipulating" Journal of Mechanical Design, ASME, v 199 n 3 september 1997. P 349-358

18. Bicchi Antonoi, Prattichizzo Domenico, Melchiorri Claudio "Force and dynamic manipulability for cooperating robot systems" IEEE international conference of Intellegent Robotd and Systems v 3 1997. P 1479-1484

19. Hsiso lin, Perng Ming-hwei "Inverse kinematic solutions for parallel robots" Midwest symposium on circuits and systems v 1 1997. P 501-504

20. Ramdane-Cherif A, Meddah D Y, Drouin m "Inverse kinematic solution based on Lyapunov fuction for redundant and non-redundant robots" IEEE International symposium on Computational Intelligence in Robotics and Automation 1997. P 226-231

21. Yang Guilin, Chen-Iming "Novel kinematic calibration algorithm for reconfigurable robtic systems" IEEE International conference on Robotics and Automation v 4 1997. P 3197-3202

22. Nemec B "Pseudoinverse and null space velocity contoller" IEEE International conference on Intellgent Engineering Systems 1997. P 107-111

23. Caccavale Fabrizio, Chiaverini Stefano, Siciliano Bruno "Second kinematic control of robot manipulators with Jacobian damped least square inverse" IEEE/ASME Transations of Mechatronocs v 2 n 3 September 1997. P 188-194

Chen I-Meng, Yang Guilin "configuration independent kinematics for modular robots" IEEE International conference on Robotics and Automation $v$ 2 1996. P 1440-1445 
25. Merlet Jean-Pierre "direct kinematics of planar parallel manipulators" IEEE International conference on Robotics and Automation v 4 1996. P 3744-3749

26. T.C. Yih, Kun Ji, Barry L Burks, D.S.Kwon "Inverse linematic control of LDUA and TWRMS" Conference of Robotics and remote systems 1996. P 118

27. Carrera Eliodoro, Serna Miguel A "Inverse dynamics of flexible robots" Mathematics and Computers in Simulation v 41 n 5-6 August 1996. P 485-508

28. Chung W.J, Chung W. K, Youm Y "Dynamic control of redundant manipulators using full row-rank minors of Jacobian" IEEE International conference on Robotics 1993. P 1053-1058

29. Mason Matthew $\mathrm{T}$, Lynch Kevin M "Dynamic manipulation" IEEE International conference on Robotics and systems 1993. P 152-159

30. Mayorga R V, Ressa B, Wong A K C "A kinematic design optimization of robot manipulators" IEEE International conference on Robotics and Automation v 1 1992. P 396-401 